\title{
Group 11 Precursors for Atomic Layer Deposition: Design and Synthesis of Advanced Precursors Enabled by Investigations in Thermolysis
}

\author{
by \\ Jason Philip Coyle
}

A thesis submitted to the Faculty of Graduate and Postdoctoral Affairs in partial fulfillment of the requirements for the degree of

Doctor of Philosophy

in

Chemistry

Carleton University

Ottawa, Ontario

(C) 2014, Jason Philip Coyle 


\section{Abstract}

Atomic layer deposition (ALD) of copper seed layers for electrochemical deposition of copper will likely be a step in fabricating future copper interconnects of microelectronic devices. Development of precursors for copper ALD is necessary in order to enable the deposition of suitable seed layers with acceptable properties. Copper(I) amidinate compounds are leading precursor candidates for use in industry. Modifications to their chemical structure was undertaken to improve precursor properties. Copper(I) guanidinates were synthesized and used as single source precursors for copper metal films at $225^{\circ} \mathrm{C}$. Their decomposition mechanism was investigated through solution thermolysis experiments and by unravelling gas phase fragmentation pathways. Evidence for CDI deinsertion occurring in solution was observed by NMR experiments while $\beta$ hydrogen elimination was determined as the gas phase pathway by ToF-MS and MIFTIR experiments. Both pathways were rationalized by DFT calculations. Copper(I) iminopyrrolidinate compounds were specifically designed to block CDI deinsertion and $\beta$-hydrogen elimination. As a result, copper(I) tert-butyl-imino-2,2-dimethylpyrrolidinate demonstrated superior thermal stability and adsorbed from the gas phase onto high surface $\mathrm{SiO}_{2}$ at $275^{\circ} \mathrm{C}$ with simultaneous loss of its tert-butyl group. Analogous silver(I) and gold(I) compounds were demonstrated to be robust precursors for chemical vapour deposition of metal films at deposition temperatures of $140{ }^{\circ} \mathrm{C}$ and $300{ }^{\circ} \mathrm{C}$, respectively. The lack of available monomeric copper(I) amidinate, guanidinate, and iminopyrrolidinate compounds prompted synthetic work to employ N-heterocyclic carbenes and acyclic diamino carbenes as Lewis bases in copper precursors. A large series of monomeric copper(I) hexamethyldisilazide compounds were studied and 
characterized extensively by thermal gravimetric analysis. Imidazolylidenes were unsuitable for use in copper precursors due to thermal instability. Imidazolinylidenes and formamidinylidenes fashioned several promising precursors demonstrating excellent thermal stability and good volatility. The leading candidate, 1,3-diisopropyl-imidazolin2-ylidene copper hexamethyldisilazide, had a 1 Torr vapour pressure at $149^{\circ} \mathrm{C}$ and could be heated at $130{ }^{\circ} \mathrm{C}$ for two weeks without decomposition. Copper metal films were deposited by plasma enhanced ALD at $225^{\circ} \mathrm{C}$ on $\mathrm{SiO}_{2}$. 


\section{Preface}

This preface provides full bibliographical details for each article included in this thesis, as well as whether the article is reproduced in whole or in part. Use of copyrighted material is likewise acknowledged here. When citing material from this thesis, please cite the article relevant to the chapter, if the chapter is based on a publication.

Pursuant to the Integrated Thesis policy of Carleton University, the supervisor (Seán T. Barry) and the "student" (Jason P. Coyle) confirm that the student was fully involved in setting up and conducting the research, obtaining data and analyzing results, as well as preparing and writing the material presented in the co-authored articles(s) integrated in the thesis. Additionally, the supervisor confirms the information provided by the student in this preface.

\section{Chapter 2}

Coyle, J. P.; Monillas, W. H.; Yap. G. P. A.; Barry, S. T. "Synthesis and Thermal Chemistry of Copper(I) Guanidinates" Inorg. Chem. 2008, 47, 683-689.

The article is wholly reproduced and edited for formatting and clarity of presentation. Jason P. Coyle performed all work related to synthesis and characterization of compounds, solution and gas phase thermolysis experiments, and deposition of copper films. Crystallography was preformed by Wesley H. Monillas and Glenn P. A. Yap. The article was written by Seán T. Barry.

\section{Chapter 3}

Coyle, J. P.; Johnson, P. A.; DiLabio, G. A.; Barry, S. T.; Müller, J. "Gas-Phase Thermolysis of a Guanidinate Precursor of Copper Studied by Matrix Isolation, Time-ofFlight Mass Spectrometry, and Computational Chemistry" Inorg. Chem. 2010, 49, 28442850.

The article is wholly reproduced and edited for formatting and clarity of presentation. The $\Delta \mathrm{G}$ value for carbodiimide deinsertion in reference 16 was corrected to -44.4 $\mathrm{kcal} / \mathrm{mol}$ along with an associated minor change to the body of text. Jason P. Coyle performed all work related to synthesis and characterization of compounds, TOF MS and MI FTIR experiments. Mechanistic DFT calculations were performed by Paul A. Johnson supervised by Gino A. DiLabio. Jens Müller supervised the TOF MS and MI FTIR experiments and calculated frequencies for trans-3. Writing was collaborative between all co-authors.

\section{Chapter 4}

Coyle, J. P.; Kurek, A.; Pallister, P. J.; Sirianni, E. R.; Yap, G. P. A.; Barry, S. T. "Preventing Thermolysis: Precursor Design for Volatile Copper Compounds" Chem. Commun. 2012, 48, 10440-10442. 
Coyle, J. P.; Pallister, P. J.; Kurek, A.; Sirianni, E. R.; Yap, G. P. A.; Barry, S. T. "Copper Iminopyrrolidinates: A Study of Thermal and Surface Chemistry" Inorg. Chem. 2013, 52, 910-917.

The articles are partially reproduced and edited to prevent repetition, formatting and clarity of presentation. 1 Torr values for compounds 1-4 are different from the publication because a calculation error was corrected. Partial results for the surface studies are reproduced. Jason P. Coyle performed all work related to synthesis and characterization of compounds, and thermo-gravimetric analysis. Solution thermolysis experiments were performed by Agnes Kurek. Surface studies were performed by Peter J. Pallister. Crystallography was performed by Eric R. Sirianni and Glenn P. A. Yap. Writing was collaborative between all co-authors

\section{Chapter 5}

Coyle, J. P.; Gordon, P. G.; Wells. A. P.; Mandia, D. J.; Sirianni, E. R.; Yap, G. P. A.; Barry, S. T. "Thermally Robust Gold and Silver Iminopyrrolidinates for Chemical Vapor Deposition of Metal Films" Chem. Mater. 2013, 25, 4566-4573.

The article is wholly reproduced and edited for formatting and clarity of presentation. Jason P. Coyle performed all work related to synthesis and characterization of compounds, thermogravimetric analysis, gold film deposition and some film thickness measurements by AFM. Peter G. Gordon performed depositions of the silver thin films, thin film characterization by SEM and AFM, and resistivity measurments. Adam P. Wells performed solution thermolysis experiments. David J. Mandia preformed XPS characterizations. Crystallography was performed by Eric R. Sirianni and Glenn P. A. Yap. Jason P. Coyle wrote the publication.

\section{Chapter 6}

Coyle, J. P.; Dey, G.; Sirianni, E. R.; Kemell, M. L.; Yap, G. P. A.; Ritala, M.; Leskelä, M.; Elliott, S. D.; Barry, S. T. "Deposition of Copper by Plasma-Enhanced Atomic Layer Deposition Using a Novel N-Heterocyclic Carbene Precursor" Chem. Mater. 2013, 25, 1132-1138.

The article is wholly reproduced and edited for formatting and clarity of presentation. 1 Torr values for compounds $\mathbf{1}$ and $\mathbf{2}$ are different from the publication because a calculation error was corrected. Jason P. Coyle performed all work related to synthesis and all characterizations of compounds including thermo-gravimetric analysis. PE-ALD experiments of copper thin films were performed by Seán T. Barry, characterised by Marianna L. Kemell, and supervised by Mikko Ritala and Markku Leskelä. Crystallography was performed by Eric R. Sirianni and Glenn P. A. Yap. DFT calculated surface models were performed by Gangotri Dey and supervised by Simon D. Elliott. Writing was collaborative between all co-authors. 


\section{Chapter 7}

Jason P. Coyle performed all work related to synthesis and characterization of compounds including thermo-gravimetric analysis. Crystallography of compounds $\mathbf{2}$ and 7 were performed by Eric R. Sirianni and Glenn P. A. Yap. Crystallography of compounds $\mathbf{8}$ and $\mathbf{1 0}$ were performed by Ilia Korobkov. Writing was collaborative between Jason P. Coyle and Seán T. Barry. 


\section{Acknowledgements}

I would like to thank Seán for inviting me to join his lab eight years ago. I started as a volunteer and was immediately drawn to the excitement around making new discoveries and the necessary high-level, social and technical discussions that would follow. Unhesitatingly, I continued on as a co-op, honour, master, and doctoral student, but these milestones were always secondary to just being a member of his lab. Every discovery I made in the lab was met by an equal opportunity to explore its significance new equipment was purchased; conferences were attended; stints with collaborators were funded; and partnerships in commercialization ventures were formed. Throughout my time in the Barry Lab, Seán worked endlessly to improve or start facilities that his lab, the chemistry department, and other faculties could share on top of being a popular and effective teacher. Yet, working in his lab never seemed like work, nor was it expected to. As I have realized from science, progress is easily limited by expectations. It has been a great pleasure to be mentored by someone who expected nothing, but was prepared for anything.

Although, I preferred to work solitarily in the lab, this was not considered "safe", so I would like to thank my fellow labmates over the years. Thank you Amanda, Allison, Yamile, Pete, Julie, Todd, Paul, Agnes, Peter, Dave, Matt, Sara and the many others from summer and honour projects for keeping me in awesome and "safe" company. Also, thank you for your tolerance during my most serious and silliest of times.

I would like to express my gratitude towards the Chemistry Department's technical staff and their willingness to help or advise. Thank you Keith, Tony, Jim, Peter, Susa, Tanya, Elena, Mastaneh, Graham, Daniel, and Shari for your kindness and support. 
I would also like to thank Chantelle for keeping me on track near the tail end of my studies.

Thank you to the members of the Ianoul Lab, the Manthorpe Lab, the DeRosa Lab, and the Wang Lab for generously sharing your resources and knowledge whenever I showed up at your doors.

On several occasions, I visited the lab of Dr. Jens Müller. His hospitality, support and guidance concerning gas phase and synthetic techniques had a profound impact on my development as a chemist. Of course, his students were largely responsible for the fond memories. Thank you Nora, Bid, Nick, Sam, Saeid, Elaheh, and Subhayen for welcoming and treating me as a full member of the lab. I would also like to thank Garth and Darren for saving me from instrumentation failure and Cathy for help with arranging my visits and orientation around the department.

Crystallographers were just a couple days away by FedEx in Delaware or a bus ride to the University of Ottawa. Drs. Glenn Yap, Wesley Monillas, Eric Sirianni, and Ilia Korobkov were always as keen to solve my samples as I was to send them. My syntheses were never complete without a solved structure. Thank you.

Without disclosure, I have valued my interaction with GreenCentre Canada and Parteq Innovations immensely. It was truly eye opening to work with their commercialization and patent experts.

Lastly, I would like to thank Dave and Jeff at Applied Materials for my internship experience and introduction to the ALD industry. Their lessons and knowledge were invaluable to me, as a student, and sharpened my focus after I returned to Carleton. I am 
also appreciative of the hospitality from Juhana, Wei-Min, and Satu during the brief time I spent using reactors at Picosun. 
This thesis is dedicated to my parents, Anne Elizabeth and David George Coyle. 


\section{Table of Contents}

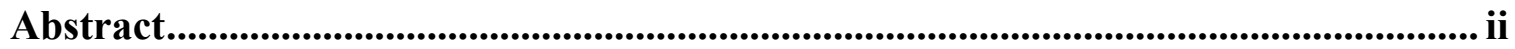

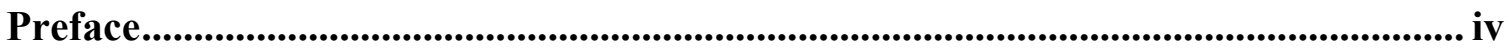

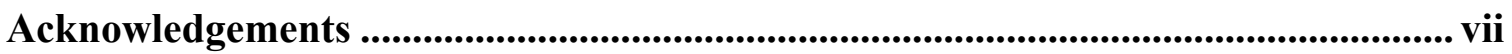

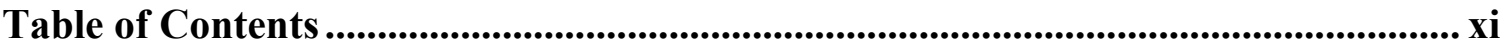

List of Tables .......................................................................................................................... xiv

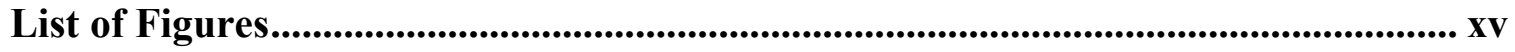

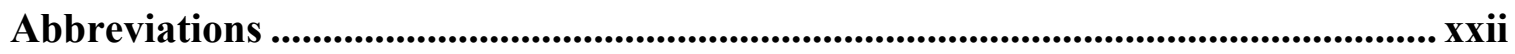

1 Chapter: Introduction ......................................................................................... 1

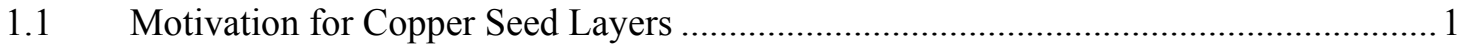

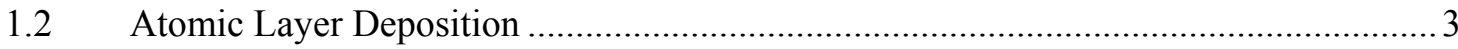

1.3 Copper Alkyl, Amido, and Alkoxide Complexes....................................................... 4

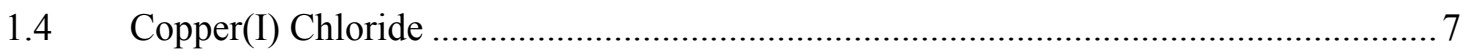

1.5 Copper Precursors for Atomic Layer Deposition ................................................ 8

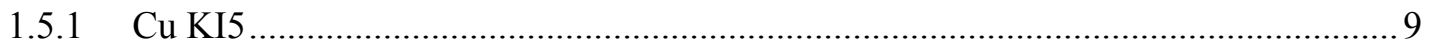

1.5.2 Bis(tri-n-butylphosphine) Copper(I) acetylacetonate ............................................ 10

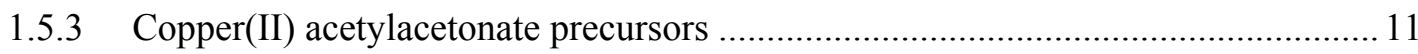

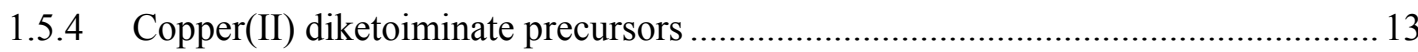

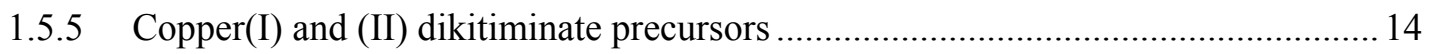

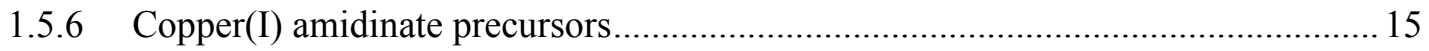

1.5.7 Copper(II) aminoalkoxide precursors .............................................................. 16

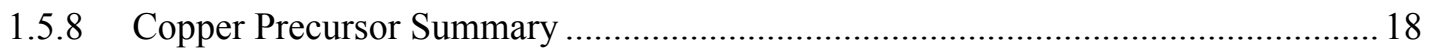

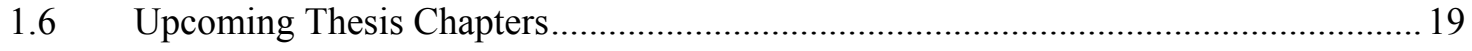

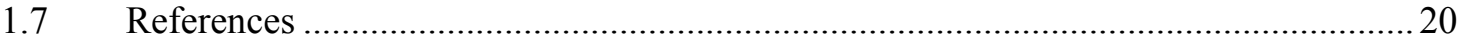

2 Chapter: Synthesis and Thermal Chemistry of Copper (I) Guanidinates .................. 27 


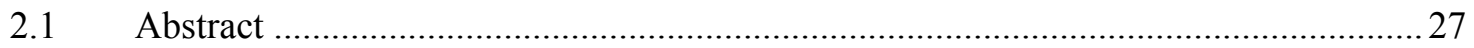

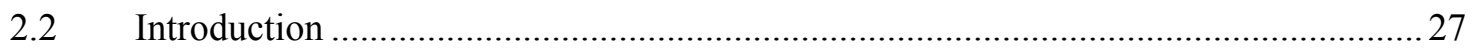

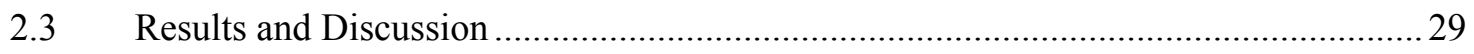

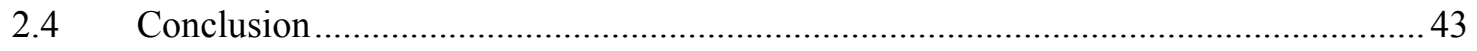

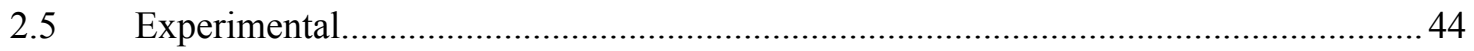

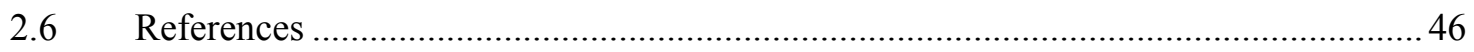

3 Chapter: Gas-Phase Thermolysis of a Guanidinate Precursor of Copper Studied by Matrix Isolation, Time-of-Flight Mass Spectrometry and Computational Chemistry ..... 49

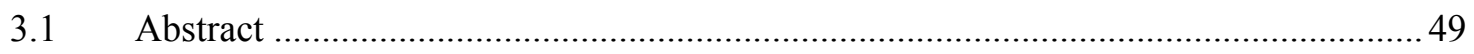

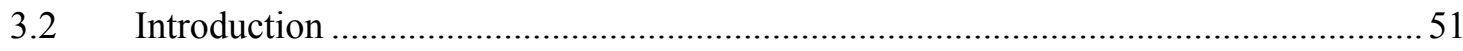

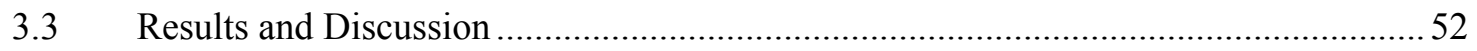

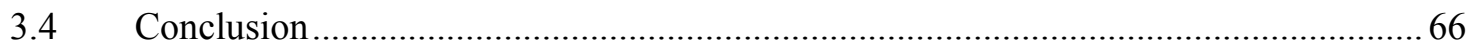

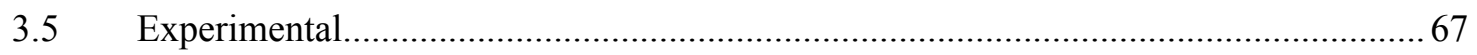

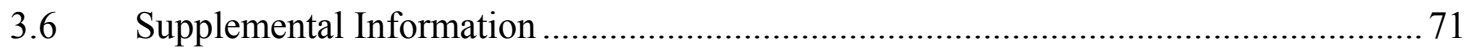

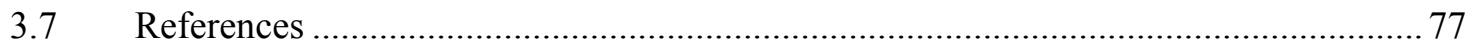

4 Chapter: Copper Iminopyrrolidinates ......................................................................... 80

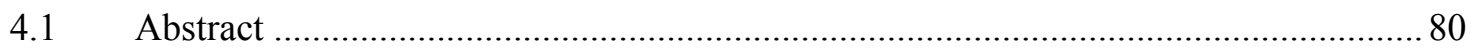

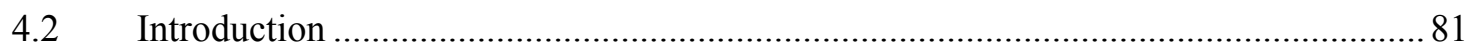

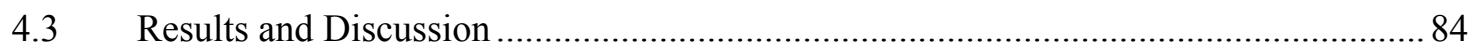

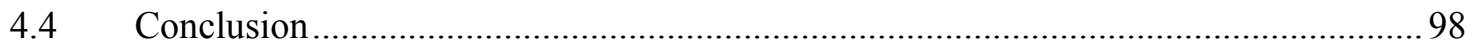

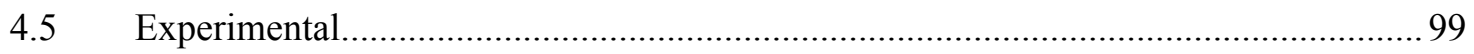

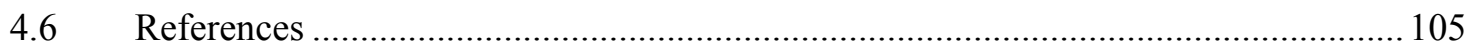

5 Chapter: Thermally Robust Gold and Silver Iminopyrrolidinates for Chemical Vapour Deposition of Metal Films ..................................................................................... 108

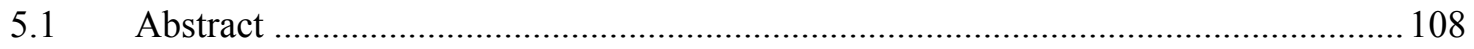

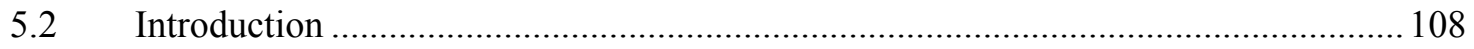




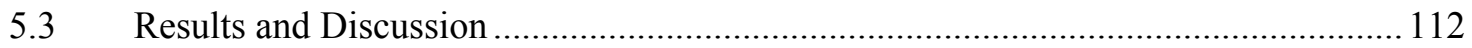

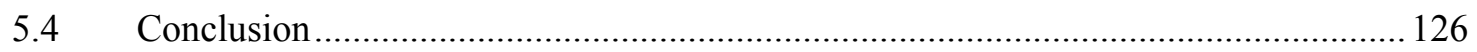

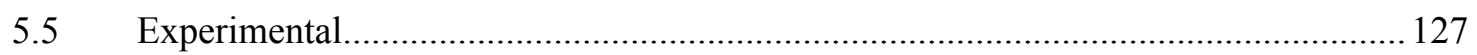

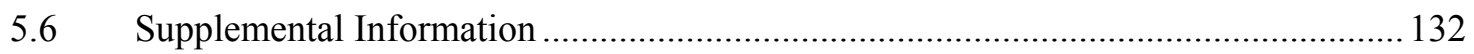

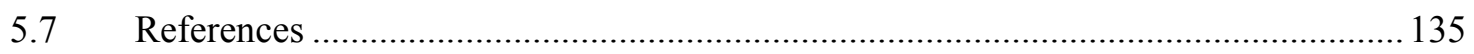

6 Chapter: Deposition of Copper by Plasma-Enhanced Atomic Layer Deposition

Using a Novel N-Heterocyclic Carbene Precursor .............................................................. 139

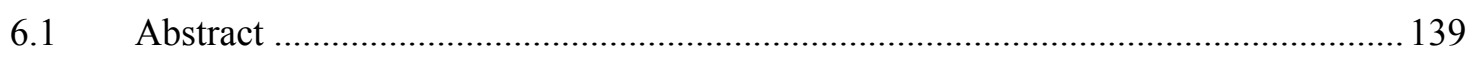

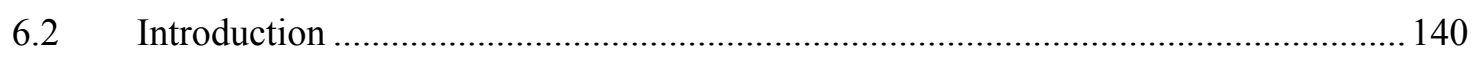

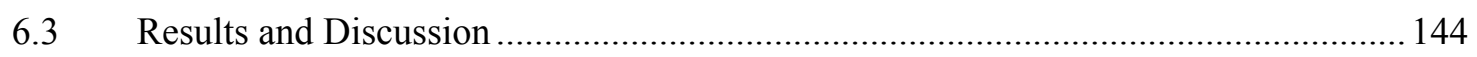

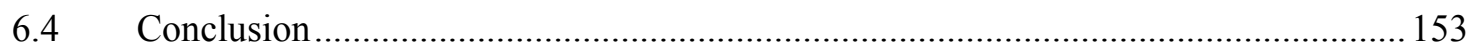

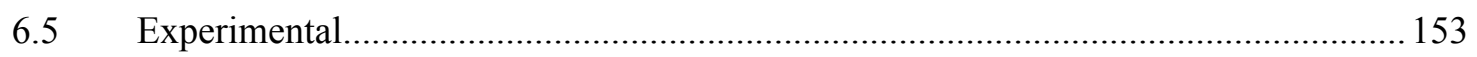

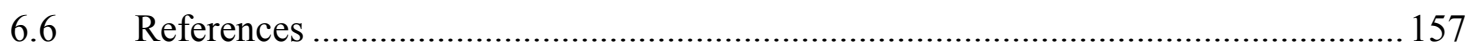

7 Chapter: Synthesis and Thermal Gravimetric Studies of Monomeric $\mathrm{Cu}(\mathrm{I}) \mathrm{N}\left(\mathrm{SiMe}_{3}\right)_{2}$ Complexes Supported by N-Heterocyclic and Acyclic Diamino Carbenes. ................... 162

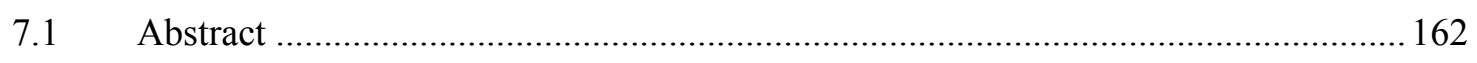

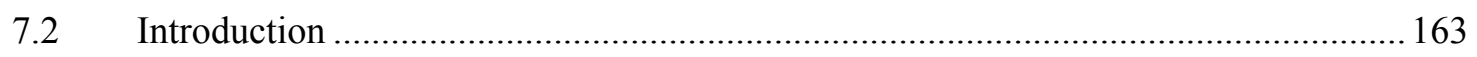

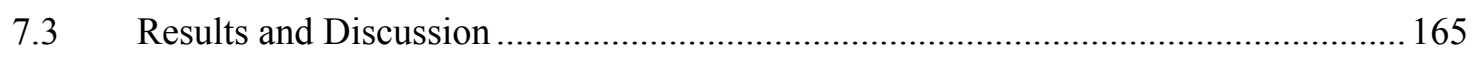

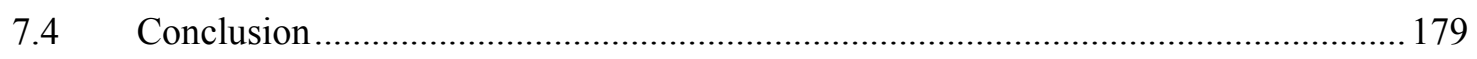

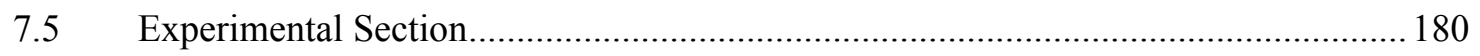

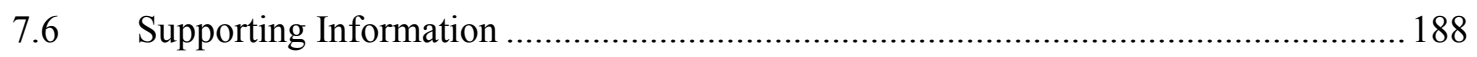

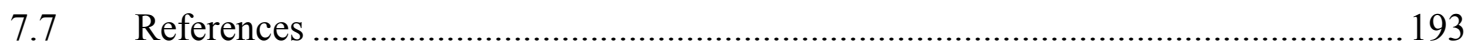

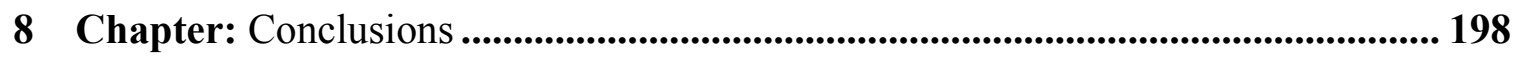

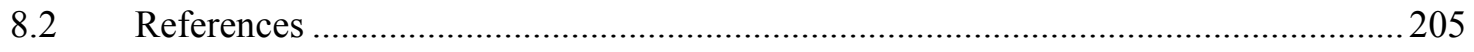




\section{List of Tables}

Table 2.1 Selected Crystal Data and Structure Refinement Parameters ........................ 32

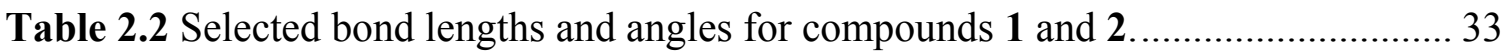

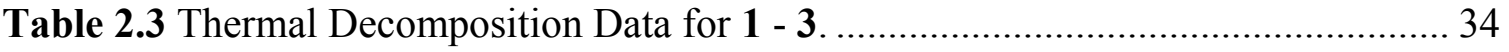

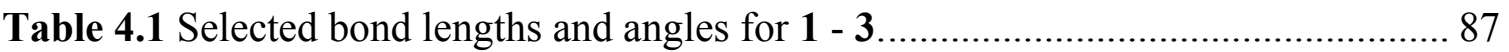

Table 4.2 Kinetic data for the thermal decomposition of $\mathbf{1}-\mathbf{4}$ at $165^{\circ} \mathrm{C}$. The "correlation

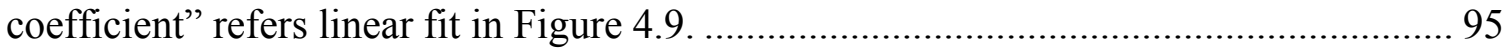

Table 5.1 Crystal Data and Refinement Details of 1 and 2..................................... 114

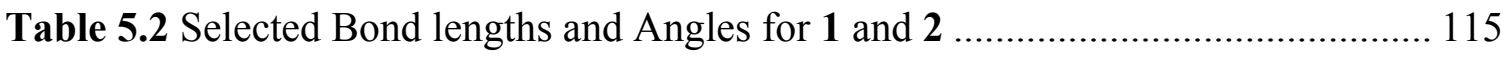

Table 6.1 Selected bond lengths and bond angles for the structures of 1 and 2.......... 146

Table 6.2 Computed Adsorption Energy $\Delta E_{\mathrm{ad}}(\mathrm{kJ} / \mathrm{mol})$ of the Precursors and Their

Probable by-Products on Two Surface Models - $\mathrm{Cu}_{55}$ with a Smooth (111) Surface and

$\mathrm{Cu}_{56}$ with One Extra Atom Making the Surface Rough. ${ }^{\mathrm{a}}$

Table 7.1 Properties of Diamino Carbene Cu(I) HMDS Compounds $\mathbf{1}-\mathbf{1 0}$

Table 7.2 Selected Bond Lenghts and Bond Angles for the Structures of 2, 3, 5, 7, 8 and 10. ${ }^{a}$ Data was previously reported and provided for convenience. ${ }^{8}$ 170

Table 7.3 Crystal Data and Structure Refinement Parameters. 188 


\section{List of Figures}

Figure 1.1 The copper ketoiminate precursor CuKI5 with a tethered vinyl-silyl Lewis base.

Figure 1.2 Copper(II) acetylacetonate, $\mathrm{R}=\mathrm{H}$; tetramethylheptanedionate, $\mathrm{R}=\mathrm{Me}$; hexafluoroacetylacetonate, $\mathrm{R}=\mathrm{F}$. .......................................................................... 12

Figure 1.3 Copper(II) 2-ethylimino-4-pentanonate ………………………………..... 13

Figure 1.4 Asymmetric copper(I) and (II) dikitiminate precursors. ............................... 14

Figure 1.5 Copper(I)- $N, N^{\prime}$-di-sec-butylacetamidinate ……………………………..... 15

Figure 1.6 Copper(II) aminoalkoxides, $\mathrm{R}=\mathrm{H}$, alkyl, fluoroalkyl................................ 17

Figure 2.1 Resonance Structures for Copper Amidinate and Guanidinate Ligands. ....... 28

Figure 2.2 Thermally Induced Carbodiimide Deinsertion............................................... 29

Figure 2.3 Single crystal X-ray structures for compounds 1 and 2. Hydrogen atoms were omitted for clarity, and the thermal ellipses are shown at $30 \%$........................................ 30

Figure 2.4 The dimer cores of (a) 1 and (b) 2, showing the twists induced by pyramidalization of the chelating nitrogens. Except for the ligands' $\mathrm{sp}^{2}$ carbon, all carbon and hydrogen atoms are omitted for clarity, and the thermal ellipses are shown at $30 \%$.

Figure 2.5 Thermogravimetric analyses of $\mathbf{1}$ (solid line), $\mathbf{2}$ (bold line), and $\mathbf{3}$ (grey line).

Figure 2.6 Thermal Decomposition of Copper Guanidinates 36

Figure 2.7 Temperature-resolved mass spectrum of 1 showing the increase in carbodiimide and a corresponding decrease in the dimethylamido diisopropyl guanidine.

Figure 2.8 Temperature-resolved mass spectrum of $\mathbf{2}$ showing the increase in carbodiimide and a corresponding decrease in the protonated triisopropyl guanidine cation

Figure 2.9 The temperature-resolved mass spectrum of $\mathbf{3}$ showing the increase in the ligand fragment " $\mathrm{C}=\mathrm{N}^{\mathrm{i}} \mathrm{Pr}$ " and a corresponding decrease in the protonated acetamidine cation. 
Figure 2.10 Scanning electron micrograph of copper films deposited by (a) 1 and (b) 2 .

Figure 2.11 X-ray diffraction spectra of copper deposited in a CVD experiment using (a) 1 and (b) $\mathbf{2}$ as a precursor. 42

Figure 3.1 Decomposition of a Copper Guanidinate via CDI Deinsertion. 51

Figure 3.2 Overview MS of the copper guanidinate 1 with thermolysis oven at $130{ }^{\circ} \mathrm{C}$ (no thermal fragmentation; bottom spectrum; $\mathrm{M}^{+}$of 1 enlarged) and at $260{ }^{\circ} \mathrm{C}$ (complete

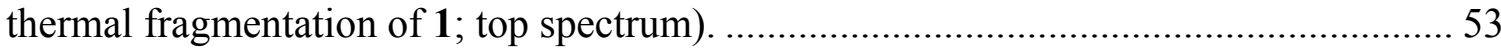

Figure 3.3 Protonated and oxidized ligand with calculated masses.............................. 54

Figure 3.4 Temperature dependence of intensities of selected ions arising from the

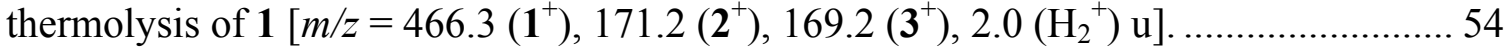

Figure 3.5 Decomposition of a Copper Guanidinate via $\beta$-hydride Elimination............ 55

Figure 3.6 Measured IR spectra of copper guanidinate 1 and its thermolysis products ( $x$ axis, $1500-1800 \mathrm{~cm}^{-1} ; y$ axis, absorbance). Spectrum A: $\mathbf{2}$ in solid argon (thermolysis oven at $260^{\circ} \mathrm{C}$ ); see Figure 3.13 for a complete IR spectrum. Spectrum B: results of a thermolysis of complex 1 at $260^{\circ} \mathrm{C}$; see Figure 3.12 for a complete IR spectrum. Spectrum C: 3 in solid argon (thermolysis oven at $260{ }^{\circ} \mathrm{C}$ ); residual amounts of $\mathbf{2}$ have been electronically eliminated; see Figure 3.14 for a complete IR spectrum.

Figure 3.7 Possible isomers of $\mathbf{3}$ and the optimized structure for trans-3 obtained with B3LYP/6-31+G(d,p)-DCP.

Figure 3.8 Calculated fragmentation pathways for precursor 1. Free energies relative to A1 for the reaction steps are shown (values in parentheses are relative to species 1)..... 62

Figure 3.9 Temperature dependences of selected ions of the thermolysis at high temperatures of $\mathbf{1}$ (spectrum B) and $\mathbf{2}$ (spectrum A) $\left[\mathrm{m} / \mathrm{z}=171.2\left(\mathbf{2}^{+}\right), 169.2\left(\mathbf{3}^{+}\right), 126.2\right.$ $\left.\left(4^{+}\right), 124.1\left(\mathbf{C D I}^{+}\right) 45.0\left(\mathrm{HNMe}_{2}{ }^{+}\right) \mathrm{u}\right]$. The intensities of masses at 169.2 and $124.1 \mathrm{u}$ were negligible in spectrum A (counts between 2 and 22 at sweep rates of $8 \times 10^{6}$ )..... 65

Figure 3.10 Carbodiimides 65

Figure 3.11 Species 1 in solid argon (oven temperature at $140{ }^{\circ} \mathrm{C}$; no decomposition; 220 $-4000 \mathrm{~cm}^{-1}$ ). Wavenumbers [ $\mathrm{cm}^{-1}$ ] of the marked IR bands are 2960.4, 2919.2, 2850.9, 2785.3, 1510.1, 1489.7, 1467.0, 1444.0, 1397.0, 1375.7, 1359.2, 1314.2, 1275.3, 1171.9, $1137.2,1053.0,783.2,733.8,457.4$

Figure 3.12 Results of a thermolysis of complex 1 at $260{ }^{\circ} \mathrm{C}\left(220-4000 \mathrm{~cm}^{-1}\right.$; same IR spectrum as Figure 3.6B). Wavenumbers $\left[\mathrm{cm}^{-1}\right]$ of the marked IR bands are 3012.8 , 
2972.8, 2939.2, 2904.6, 2874.2, 2839.4, 2794.6, 1687.9, 1668.9, 1635.1, 1626.6, 1488.4, $1452.9,1435.4,1372.5,1337.4,1261.9,1246.2,1190.7,1167.9,1144.8,1079.1,1056.6$, 1003.2, 942.5 . 72

Figure 3.13 Species 2 in solid argon (thermolysis oven at $260{ }^{\circ} \mathrm{C}$; no decomposition; 220 $-4000 \mathrm{~cm}^{-1}$; same IR spectrum as Figure 3.6A). The sample of 2 contained residual amounts of $i \operatorname{PrNCN} i \operatorname{Pr}(\mathbf{C D I})$, which were electronically eliminated from the IR spectrum of 2. Wavenumbers $\left[\mathrm{cm}^{-1}\right]$ of the marked IR bands are 3408.7, 3013.9, 2975.9, 2941.7, 2873.5, 2836.6, 2811.4, 2794.2, 1646.0, 1486.2, 1457.0, 1435.1, 1367.3, 1348.3, $1308.1,1254.3,1169.3,1146.8,1129.2,1058.7,1031.4,937.3$

Figure 3.14 Species 3 in solid argon (thermolysis oven at $260{ }^{\circ} \mathrm{C}$; no decomposition; 220 $-4000 \mathrm{~cm}^{-1}$; same IR spectrum as Figure 3.6C). The sample of 2 contained small amounts of $i \operatorname{PrNCN} i \operatorname{Pr}(\mathrm{CDI})$, which were electronically eliminated from the spectrum of 3. Wavenumbers $\left[\mathrm{cm}^{-1}\right]$ of the marked IR bands are 3012.9, 2973.4, 2939.3, 2874.6, $2839.5,2794.5,1687.9,1668.9,1634.9,1626.7,1488.5,1452.2,1436.8,1372.3,1337.3$, $1261.9,1246.1,1190.7,1167.6,1144.6,1079.4,1003.2,942.5$. 74

Figure 3.15 Results of a thermolysis of complex 2 at $700{ }^{\circ} \mathrm{C}\left(220-4000 \mathrm{~cm}^{-1}\right)$. Wavenumbers $\left[\mathrm{cm}^{-1}\right]$ of the marked IR bands are (assignments in parentheses) 3306.0, 2985.6 CDI), 2975.0 $\left(\mathrm{HNMe}_{2}\right), 2940.6\left(\mathrm{HNMe}_{2}\right), 2874.4\left(\mathrm{HNMe}_{2}\right), 2832.3\left(\mathrm{HNMe}_{2}\right)$, $2789.5\left(\mathrm{HNMe}_{2}\right), 2123.5$ (CDI; the structure of this IR band changes depending on the way CDI is deposited in matrices; see reference L. C. Ziffle, A. P. Kenney, S. T. Barry and J. Müller, Polyhedron, 2008, 27, 1832-1840.), 1645.9 (most intense peak of 2), 1457.6 ( $\left.\mathrm{HNMe}_{2}\right), 1384.5$ (CDI), 1368.4 (CDI), 1319.6 (CDI), 1306.7 (CDI), 1167.7 (CDI), 1150.4 ( $\left.\mathrm{HNMe}_{2}\right), 1119.8$ (CDI), 1020.9 ( $\left.\mathrm{HNMe}_{2}\right)$, 935.8 (CDI), 908.6, 728.6 (most intense peak of $\mathrm{HNMe}_{2}$ ), 632.6 (CDI). The assignment of IR bands to $\mathrm{HNMe}_{2}$ is based on an independently measured IR spectrum of matrix-isolated $\mathrm{HNMe}_{2}$ in argon (B. Wittig, J. Müller, unpublished results).......................................................................... 75

Figure 3.16 Results of a thermolysis of complex 1 at $700{ }^{\circ} \mathrm{C}\left(220-4000 \mathrm{~cm}^{-1}\right)$. Small amounts of $\mathbf{2}$ and $\mathbf{3}$ were electronically eliminated from this spectrum. Wavenumbers $\left[\mathrm{cm}^{-1}\right]$ of the marked IR bands are (assignments in parentheses) 2985.4, $2975.2\left(\mathrm{HNMe}_{2}\right)$, 2940.7 $\left(\mathrm{HNMe}_{2}\right), 2874.4\left(\mathrm{HNMe}_{2}\right), 2832.3\left(\mathrm{HNMe}_{2}\right), 2789.5\left(\mathrm{HNMe}_{2}\right), 2127.7,1649.2$, $1483.3\left(\mathrm{HNMe}_{2}\right), 1457.3\left(\mathrm{HNMe}_{2}\right), 1439.1\left(\mathrm{HNMe}_{2}\right), 1386.9,1369.6,1337.9,1323.8$, 1230.4, 1169.0, $1150.3\left(\mathrm{HNMe}_{2}\right), 1106.0,1021.0\left(\mathrm{HNMe}_{2}\right), 929.1\left(\mathrm{HNMe}_{2}\right), 866.6$, 728.7 (most intense peak of $\mathrm{HNMe}_{2}$ ), 626.1. Note: The marked, but unassigned IR bands could be tentatively assigned to the new carbodiimide 4 . However, an unambiguous characterization of $\mathbf{4}$ on the basis of the IR data alone is impossible. The IR band at $1649.2 \mathrm{~cm}^{-1}$ could be caused by the $\mathrm{C}=\mathrm{C}$ stretching mode of 4 (see Figure 3.10 ). The assignment of IR bands to $\mathrm{HNMe}_{2}$ is based on an independently measured IR spectrum of matrix-isolated $\mathrm{HNMe}_{2}$ in argon (B. Wittig, J. Müller, unpublished results).................. 76

Figure 4.1 Two Thermal Decomposition Pathways For a Copper(I) Guanidiante Species. 82 
Figure 4.2 Series of Amidinate-Type Ligands with Differing Numbers of $\beta$-Hydrogens, Shown As Their Corresponding Copper(I) Compounds 83

Figure 4.3 The synthetic procedure for producing the tert-butyl-imino-2-

Figure 4.4 Structure of 1, shown with the hydrogens removed for clarity. The thermal ellipsoids are at $30 \%$.

Figure 4.5 Structure of (a) 3 and (b) 4 , shown with the hydrogens removed for clarity. The thermal ellipsoids are at $30 \%$.

Figure 4.6 Thermal stress test of compounds (a) 3 showing no change in residual mass with increasing sample mass, and (b) $\mathbf{1}$, showing increasing residual mass with increasing initial sample mass.

Figure 4.7 Thermal stress test trends for compounds 1-4 ......................................... 92

Figure 4.8 Evaporation kinetics of 1-4 by TGA.......................................................... 93

Figure 4.9 Thermal decomposition of $\mathbf{1 - 4}$ in $\mathrm{C}_{6} \mathrm{D}_{6}$ at $165^{\circ} \mathrm{C}$ over 21 days.................. 94

Figure 4.10 Reactivity of 1 with silica: (a) shows the SS- ${ }^{13} \mathrm{C}$ NMR of silica after deposition of $\mathbf{1}$ at $275^{\circ} \mathrm{C}$ (top trace) compared to the HR ${ }^{13} \mathrm{C}$ NMR of $\mathbf{1}$ in deuterated benzene (bottom trace). (b) shows the HR- ${ }^{1} \mathrm{H}$ NMR of the surface species etched from the silica with $\mathrm{D}_{2} \mathrm{O}$ (in $\mathrm{D}_{2} \mathrm{O}$; top trace) compared to the HR- ${ }^{1} \mathrm{H}$ NMR of $\mathbf{1}$ in $\mathrm{CDCl}_{3}$ (bottom trace). (c) shows the proposed nucleation and chemisorption of $\mathbf{1}$ at a silanol

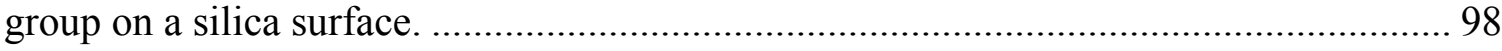

Figure 5.1 Amidinates, Guanidinates, and Iminopyrrolidinates Used as Ligands For Precursors in CVD and ALD (R, R' = any alkyl group).

Figure 5.2 Synthesis of Silver(I) and Gold(I) tert-butyl-imino-2,2-dimethylpyrrolidinate.

Figure 5.3 Molecular diagram of $\mathbf{2}$ with thermal ellipsoids at 30\% probability. H-atoms omitted for clarity. Ag analogue compound $\mathbf{1}$ is isomorphic with $\mathbf{2}$. 114

Figure 5.4 Thermogravimetric analysis of 1 (black) and $\mathbf{2}$ (gray). Experiments were run under $1 \mathrm{~atm}$ of nitrogen with a ramp rate of $10{ }^{\circ} \mathrm{C} / \mathrm{min}$. Sample sizes for $\mathbf{1}$ and $\mathbf{2}$ were $10.49 \mathrm{mg}$ and $13.65 \mathrm{mg}$, respectively. 116

Figure 5.5 . Isothermal TGA of (a) 1 and (b) 2 . The furnace was heated at $40{ }^{\circ} \mathrm{C} / \mathrm{min}$ up to the isothermal temperature. Isothermal temperatures are indicated in the right hand legend $\left({ }^{\circ} \mathrm{C}\right)$. Samples sizes were between $3.10 \mathrm{mg}$ to $3.67 \mathrm{mg}$. 
Figure 5.6 Differential scanning calorimetry of 1, 2, and $\mathbf{3}$ in hermetically sealed aluminum pans. Temperature ramp was $10^{\circ} \mathrm{C} / \mathrm{min}$ up to $325^{\circ} \mathrm{C}$. Sample masses were $1.066 \mathrm{mg}, 1.625 \mathrm{mg}$, and $3.790 \mathrm{mg}$ for 1, 2, and 3, respectively. Exotherms are up. .... 118

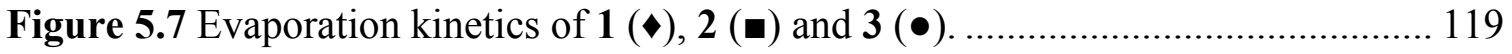

Figure 5.8 Decomposition of $\mathbf{1}$ and $\mathbf{2}$ in solution $\left(\mathrm{C}_{6} \mathrm{D}_{6}\right)$ measured by ${ }^{1} \mathrm{H}$ NMR......... 120

Figure 5.9 Film thickness determined from the CVD of $\mathbf{1}$ (bottom) and $\mathbf{2}$ (top) at different furnace temperatures. Depositions with 1 consumed $50 \mathrm{mg}-60 \mathrm{mg}$ of compound. Depositions from 2 consumed varying amounts: $13.9 \mathrm{mg}\left(300^{\circ} \mathrm{C}\right) ; 21.8 \mathrm{mg}$ $\left(350{ }^{\circ} \mathrm{C}\right) ; 5.9 \mathrm{mg}\left(400{ }^{\circ} \mathrm{C}\right) ; 20.6 \mathrm{mg}\left(450^{\circ} \mathrm{C}\right) ; 23.5\left(500^{\circ} \mathrm{C}\right)$.

Figure 5.10 SEM images for thin films deposited from (a, b) 1 and (c, d) 2 at $200{ }^{\circ} \mathrm{C}$ and $350{ }^{\circ} \mathrm{C}$, respectively. Images from the CVD of $\mathbf{1}$ were measured (a) $0.75 \mathrm{~cm}$ and (b) 7.75 $\mathrm{cm}$ from the precursor inlet. Images from the CVD of 2 were measured (a) $5 \mathrm{~cm}$ and (b) $11 \mathrm{~cm}$ from the precursor inlet. 123

Figure 5.11 Film thickness determined from timed CVD experiments of 1 under a low flow of $\mathrm{N}_{2}$ at $300{ }^{\circ} \mathrm{C}$ with a system pressure of 100 mtorr. 125

Figure 5.12 XPS survey spectrum of $\mathrm{Ag}$ on $\mathrm{Si}\left(\begin{array}{lll}1 & 0 & 0\end{array}\right)$ substrate as-deposited (blue), after 1 sputtering cycle (green), after 2 sputtering cycles (red), and after 3 sputtering cycles (black). $\mathrm{Ar}^{+}$sputtering cycles lasted $2 \mathrm{~min}$ and were done under base Ar pressures of $1 \mathrm{x}$ $10^{-5}$ Torr.

Figure 5.13 XPS survey spectrum of Au on Si ( $\left.\begin{array}{lll}1 & 0 & 0\end{array}\right)$ substrate as deposited (black), after 1 sputtering cycle (red), after 2 sputtering cycles (green), after 3 sputtering cycles (blue), and after 4 sputtering cycles (cyan). $\mathrm{Ar}^{+}$sputtering cycles lasted 2 min and were done under Ar pressures of $1 \times 10^{-5}$ Torr.

Figure 6.1 The structures of 1,3-diisopropyl-imidazolin-2-ylidene copper hexamethyldisilazide (1) and 4,5-dimethyl-1,3-diisopropyl-imidazol-2-ylidene copper hexamethyldisilazide (2).

Figure 6.2 Thermogravimetric analyses and evaporation rates of $\mathbf{1}$ (black) and $\mathbf{2}$ (grey) run at $10{ }^{\circ} \mathrm{C} / \mathrm{min}$ ramp rate, with $10{ }^{\circ} \mathrm{C}$ increments for the stepped isotherm used to determine evaporation rate

Figure 6.3 The X-ray structure of 1 (left) and 2, (right), with $\mathrm{H}$-atoms and minor disordered contributions omitted for clarity and at $30 \%$ probability ellipsoids.

Figure 6.4 ALD saturation curve for $\mathbf{1}$ as the pulse length of $\mathbf{1}$ was varied. 148

Figure 6.5 Scanning electron micrographs of deposited copper films. (a) Plan view of copper nanoparticles deposited using a $1 \mathrm{~s}$ pulse length. (b) Plan view of crystalline 
copper deposited using a 4 s pulse length. (c) Plan view of crystalline copper deposited using a 6 s pulse length. (d) A profile of crystalline copper deposited using a 6 s pulse length

Figure 6.6 Optimized geometries of (a) 2 molecularly physisorbed on a smooth $\mathrm{Cu}$ (111) surface; (b) $\mathrm{CuN}\left(\mathrm{SiMe}_{3}\right)_{2}$ chemisorbed on the smooth surface; (c) NHC from 2 chemisorbed onto a rough surface; (d) NHC from 2 physisorbed on the smooth surface.

Figure 7.1 Structural differences between 1,3-diisopropyl-imidazolin-2-ylidene copper hexamethyldisilazide (5) and 4,5-dimethyl-1,3-diisopropyl-imidazol-2-ylidene copper hexamethyldisilazide (3)

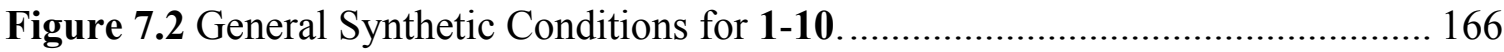

Figure 7.3 Synthesis of 4,5-dimethyl-1,3-ditertbutyl-imidazolinium chloride............. 167

Figure 7.4 Molecular diagram of 7 (left) and 8 (right) with thermal ellipsoids at $30 \%$ probability. H-atoms omitted for clarity.

Figure 7.5 Imidazolylidenes and Imidazolinylidenes, N-heterocyclic Carbenes (NHC); and the Formamidinylidenes, Acyclic Diamino Carbenes (ADC), Evaluated in Monomeric $\mathrm{Cu}(\mathrm{I}) \mathrm{HMDS}$ Complexes 1-10.

Figure 7.6 Thermal gravimetric analysis of 2, 3, 5, and 9. Experiments were run under 1 atm nitrogen with a ramp rate of $10^{\circ} \mathrm{C} / \mathrm{min}$. Sample sizes were between $5-7 \mathrm{mg}$...... 172

Figure 7.7 Thermal Decomposition of $\left[\mathrm{I}^{t} \mathrm{Bu} u_{2}\right] \mathrm{Cu}(\mathrm{I}) \mathrm{HMDS}(\mathbf{2})$ via Deportonation of the Unsaturated NHC Backbone and Elimination of Hexamethyldisilazane.

Figure 7.8 TGA thermal stress test for $3-10$. The heating rate was $10{ }^{\circ} \mathrm{C} / \mathrm{min}$ for each experiment. The smallest sample sizes were $1.3 \mu \mathrm{mol}(\sim 5 \mathrm{mg})$ and the largest were 15.9 $\mu \mathrm{mol}(\sim 60 \mathrm{mg})$.

Figure 7.9 Isothermal rates of vaporization for $\mathbf{3}-\mathbf{1 0}$ under 1 atm of nitrogen 178

Figure 7.10 Isothermal TG experiment of compound 2. An isotherm at $110{ }^{\circ} \mathrm{C}$ was maintained until mass loss was negligible, followed by heating at a rate of $10^{\circ} \mathrm{C} / \mathrm{min} .189$

Figure 7.11 TG experiments for the imidazolinylidene $\mathrm{Cu}(\mathrm{I})$ HMDS compounds 4 - 7 under $1 \mathrm{~atm} \mathrm{~N}_{2}$. The heating rate was $10{ }^{\circ} \mathrm{C} / \mathrm{min}$ and sample masses were $4-6 \mathrm{mg}$... 190

Figure 7.12 TG experiments for the formamidinylidene $\mathrm{Cu}(\mathrm{I}) \mathrm{HMDS}$ compounds $\mathbf{8}$ - 10 under $1 \mathrm{~atm} \mathrm{~N}_{2}$. The heating rate was $10{ }^{\circ} \mathrm{C} / \mathrm{min}$ and sample masses were $4-6 \mathrm{mg}$... 191 
Figure 7.13 TGA thermal stress test for compound 10 illustrating thermal decomposition. The heating rate was $10{ }^{\circ} \mathrm{C} / \mathrm{min}$ and sample masses are shown top right. ................... 192 


\section{Abbreviations}

$\mathrm{ADC}$ - acyclic diamino carbenes

AFM - atomic force microscopy

ALD - atomic layer deposition

CDI - N,N'-diisopropylcarbodiimide

$\mathrm{CMP}$ - chemical mechanical polishing

CVD - chemical vapour deposition

DCP - dispersion-correcting potentials

DFT - density functional theory

DSC - differential scanning calorimetry

ECD - electrochemical deposition

EDS - energy dispersive X-ray spectroscopy

GC-MS - gas phase chromatography mass spectrometry

HMDS - hexamethyldisilazide

HR-NMR - high resolution nuclear magnetic resonance

$\mathrm{NHC}-\mathrm{N}$-heterocyclic carbenes

NMR - nuclear magnetic resonance

MI FTIR - matrix isolation fourier transform infrared spectroscopy

MS - mass spectrometry

PE-ALD - plasma enhanced atomic layer deposition

PVD - physical vapour deposition

P-XRD - powder X-ray diffraction

QMS - quadrupole mass spectrometer 
RGA - residual gas analyzer

SEM - scanning electron microscopy

SS-NMR - solid state nuclear magnetic resonance

TEM - transmission electron spectroscopy

TGA - thermal gravimetric analysis

TOF MS - time of flight mass spectrometry

VTMS - vinyltrimetylsilane

XPS - X-ray photoelectron spectroscopy 


\section{Chapter: Introduction}

\subsection{Motivation for Copper Seed Layers}

Copper replaced aluminum as the conductive material in integrated circuits due to a lower resistivity and a higher resistance to electro-migration stress. ${ }^{1}$ The performance figure of merit for an integrated circuit is its " $\mathrm{RC}$ " time delay which is defined by the resistivity $(\mathrm{R})$ of the conductive wires and the capacitance $(\mathrm{C})$ of the insulating medium. ${ }^{2}$ The switch from aluminum to copper occurred once the density of features and components impeded the speed and frequency at which electrical signals could be transmitted. Moore's law correctly predicted the doubling of transistors every two years within an integrated circuit which has guided research and developments efforts. ${ }^{3}$ To allow for the exponential increase of the transistor density the physical dimension of each transistor was reduced. Moore's law provided guidelines for the down scaling of transistor components and the hierarchical wiring structure. ${ }^{4}$

The replacement of aluminum with copper required a major reversal of fabrication techniques. ${ }^{5}$ Aluminum is easily patterned through metal etch processes due to the volatility of aluminum chlorides and fluorides. Aluminum wires were etched from sputtered thin films with no considerations required for the conformality of the metal deposition process. After patterning of the aluminum film, the negative space was infilled with a low-k dielectric and chemical mechanical polishing (CMP) removes the excess dielectric material. For copper, a metal etch process was not feasible due to the low volatility of its metal halides; therefore, the Damascene and dual-Damascene processes were developed. ${ }^{6}$ Conceptually, the Damascene processes are the reverse of the aluminum metal etch process. ${ }^{7}$ A blanket dielectric film is patterned through 
photolithographic methods followed by infilling by electrochemical deposition of copper (EDC). ${ }^{8}$ The success of a Damascene process relies on a conformal, highly conductive, void free copper seed layer. Additionally, copper interconnects require a barrier and an adhesion layer to prevent diffusion of the copper atoms into the dielectric material and void formation due to electromigration. ${ }^{9}$ Currently, a TaN/Ta thin film deposited by physical vapour deposition (PVD) techniques is used as a barrier and adhesion layer in today's integrated circuit production line. ${ }^{1}$ The copper seed layer is deposited by PVD, as well. The conformality of these films within current aspect ratios across all levels of the hierarchical wire structure has been acceptable.

Conformality and total thickness of the liner films need to be addressed as the size regime moves towards the $10 \mathrm{~nm}$ technology node. ${ }^{1}$ Current advanced techniques deposit barrier/adhesion layers which would account for $40 \%$ of the total area of a trench at the $10 \mathrm{~nm}$ node. The barrier/adhesion materials have higher resistivity than copper and would increase the overall wire resistance, which is undesirable. Additionally, the subconformal profiles of liner materials deposited by PVD can result in voids and seam defects after electrochemical deposition of copper. Atomic layer deposition (ALD) of all liner materials is being investigated as a possible deposition technique for future technology nodes. Adoption of an entirely new liner system is likely since it is often found that material performance differs depending on the deposition technique. For example, the barrier performance of PVD TaN films might not be reproducible by ALD TaN. ${ }^{10}$ Secondly, Ta cannot be deposited by a suitable ALD process which has led to the proposition of $\mathrm{Ru}$ as an adhesion and seed layers for copper interconnects. ${ }^{11}$ Copper ALD 
processes and precursors continue to be a relevant research area for the continued implementation of copper seed layers in the future of interconnect based devices.

\subsection{Atomic Layer Deposition}

ALD is a gas phase, thin film deposition technique that achieves highly conformal coatings in a layer by layer manner. ${ }^{12}$ The success of an ALD process relies entirely on the self-limiting nature of the precursors' surface reactions. A minimum of two precursors are required to achieve the characteristic growth rate of ALD which is reported in units of angstroms per cycle $(\AA /$ cycle $) .{ }^{13}$ Adsorption of the metal containing precursor typically occurs through a chemisorption process. This step deposits a surface species that is capable of blocking further uptake of gaseous precursor and is referred to as a self-limiting monolayer. The second precursor reacts with the monolayer to deposit the target material. In some cases, particularly for metal films, the second precursor plays a crucial role in desorbing any remaining fragments to refresh the surface for the next chemisorption step. Growth rates for ALD tend to be less than the thickness of a monolayer (i.e. $\sim 2 \AA$ for a copper monolayer) of the target material due to bulky surface species blocking nucleation sites. Growth rates larger than a monolayer of the target material generally are characterized as having a chemical vapour deposition (CVD) component which indicates a deviation from self-limited growth behavior.

Three main criteria outline the design of a copper precursor capable of meeting the requirements for industrial application. These criteria centre on the precursor's volatility, thin film deposition chemistry, and chemical composition. The first two criteria are temperature dependent preferably met at low temperatures to reduce potential issues related to thermal stability and thin film morphology. The precursor needs to generate 
sufficient vapour pressure at a temperature where negligible decomposition or molecular rearrangement occurs. Precursor chemisorption and reactivity of the surface species to copper metal at appreciable surface coverages will enable an acceptable growth rate. Lastly, the precursor molecule must not contain atoms that contaminate or degrade the barrier and adhesion layers, increase the resistance of the copper interconnect, or compromise the longevity of the microelectronic device.

Processes for ALD have benefitted greatly from precursors sourced from known homoleptic compounds such as alkyl, alkoxide, amido and halides (for example; $\left.\mathrm{Ti}\left(\mathrm{O}{ }^{\mathrm{i}} \mathrm{Pr}\right)_{4}, \mathrm{AlMe}_{3}, \mathrm{SiCl}_{4}, \mathrm{Ta}\left(\mathrm{NMe}_{2}\right)_{5}\right) .{ }^{14}$ These compounds are well studied, readily available for many elements and typically possess suitable vapour pressure due to their low molecular masses. When determining an ALD process employing a known compound as a precursor, a more focused study on the characteristics of film growth is possible. Preferably, one need not consider if a precursor is remaining intact while being transported to the reaction chamber. Similar homoleptic compounds of copper have not been reported in ALD research primarily due to thermal instability and low vapour pressure due to a high degree of oligomerization.

\subsection{Copper Alkyl, Amido, and Alkoxide Complexes}

Copper(I) alkyl complexes (CuR, $\mathrm{R}=\mathrm{Me}, \mathrm{Et}, \mathrm{Pr}, n \mathrm{Bu})$ can be generated by reaction of copper(I) or (II) halides with lithium, Grignard, or zinc reagents. ${ }^{15,16,17}$ When isolated as pure solids they are explosive, which necessitates storage at sub-zero temperatures. The stability of copper(I) alkyl complexes in solution is solvent dependent showing higher stability in coordinating solvents. ${ }^{15}$ Decomposition mechanisms via homolectic cleavage of the copper-carbon bond ${ }^{18}$ or $\beta$-hydride elimination ${ }^{19}$ have been 
studied in complexes stabilized with $n \mathrm{Bu}_{3} \mathrm{P}$. Structural characterization of homoleptic copper(I) alkyl complexes has been inhibited due to their highly unstable nature and tendency to precipitate from reaction solvents. Copper(I) alkyl complexes likely adopt a high order oligomeric structure which would account for their non-volatile nature despite their having low molecular masses. Copper(II) alkyl complexes have only been described as metastable species with synthetic attempts resulting in the isolation of $\mathrm{Cu}(\mathrm{I})$ products. $^{16}$

Copper(I) amide complexes are significantly more stable and have been structurally and thermally characterised. $\mathrm{CuNR}_{2}$ (for example; $\mathrm{R}=\mathrm{Me}, \mathrm{Et}, n \mathrm{Bu}, \mathrm{SiMe}_{3}$ ) complexes can be generated through salt metathesis between copper halides and lithiated amines $^{20,21,22}$ or, by a very appealing route, prepared from primary and secondary amines with use from copper(I) mesityl as an efficient metalation reagent. ${ }^{23}$ These complexes have been characterised as exclusively tetramers in solution and the solid state. Each copper atom is two coordinate with each amido ligand bridging two copper atoms in a $\mathrm{Cu}_{4} \mathrm{~N}_{4}$ eight-membered ring. This arrangement is common throughout the alkyl, amido, and alkyloxy complexes for copper(I); however it is most persistent within the amido category as these complexes do not readily react with external nucleophiles compared to the alkyl or alkoxy complexes. ${ }^{21}$ This is perhaps indicative of a higher degree of donor bonding from the nitrogen lone pair to each $\mathrm{Cu}$ atom pair. ${ }^{20}$ Studies support $\beta$-hydride abstraction as a mechanism for thermal decomposition. ${ }^{23}$ Several copper(I) amide complexes were decomposed in tetralin solutions heated between $90-140{ }^{\circ} \mathrm{C}$ producing the decomposition products of copper metal, the parent amine and the corresponding imine. 


$$
\left[\mathrm{CuNR}_{2}\right]_{4} \rightarrow \mathrm{Cu}+\mathrm{HNR}_{2}+\mathrm{NRR}^{\prime} ; \text { where } \mathrm{R}^{\prime}=\mathrm{R}-\beta \mathrm{H}
$$

Volatility has only been demonstrated for $\left[\mathrm{CuN}\left(\mathrm{SiMe}_{3}\right)\right]_{4}$ which is the most thermally stable tetramer yet possesses the highest molecular weight. ${ }^{22}$

Copper(I) alkoxide complexes have demonstrated a large range of thermal stability showing a dependence on structure. ${ }^{24}$ For example, $[\mathrm{CuOMe}]_{4}$ decomposes explosively at room temperature ${ }^{25}$ while $\left[\mathrm{CuOCMe}_{3}\right]_{4}$ is stable up to $170{ }^{\circ} \mathrm{C} .{ }^{26} \mathrm{In}$ addition, copper(II) alkoxide complexes are sufficiently stable to be isolated and characterised which is a notable difference compared to the missing copper(II) alkyl and amide complexes. $^{27,28,29}$ Synthesis of stable copper(I) alkoxides and all copper(II) alkoxides can be accomplished by salt metathesis. The less stable copper(I) alkoxides were synthesised using copper methyl or mesityl as metalation reagents with alcohols. ${ }^{24}$ Copper(I) alkoxide complexes decompose to produce metallic copper via $\beta$-hydride abstraction or copper-oxygen bond homolysis depending on the structure of the alkoxide. ${ }^{24}$ Mechanisms for decomposition of copper(II) complexes have not been thoroughly investigated, but results indicate the an opposite structural dependence on thermal stability is observed compared to copper(I). ${ }^{28}$ Notably, disproportionation of copper(I) to (II) has not been observed for the alkoxides. In fact, decomposition of copper(II) alkoxides generate the copper(I) compounds when this is an adequately stable species. ${ }^{26}$ Oxidation (by oxygen or peroxides) of copper(I) alkoxides to copper(II) is possible. ${ }^{24,26} \mathrm{CVD}$ of copper metal films from $\left[\mathrm{CuOCMe}_{3}\right]_{4}$ has been demonstrated at deposition temperatures of $400{ }^{\circ} \mathrm{C}$ under a system pressure of $10^{-5}$ Torr. $^{30}$ The precursor vapours are generated at $100{ }^{\circ} \mathrm{C}$. Polycrystalline films were deposited without a reducing agent and contained $5 \%$ oxygen and $<1 \%$ carbon impurities consistent with the 
proposed mechanism of $\mathrm{Cu}-\mathrm{O}$ bond homolysis. Copper(II) alkoxide complexes are polymeric $^{29}$ and non-volatile ${ }^{28}$ and not useful as precursors.

\subsection{Copper(I) Chloride}

Copper halides are insoluble solids possessing extended polymeric structures. Melting points for all the halide compounds of both oxidation states are around $500{ }^{\circ} \mathrm{C}$ and boiling points well above $1000{ }^{\circ} \mathrm{C}$ often associated with decomposition. Surprisingly, copper(I) chloride has been used to test whether copper halides are suitable as precursors for ALD. Copper(I) chloride generates sufficient vapour pressure when heated to 390 at 10 mbar. The major gaseous species is $\mathrm{Cu}_{3} \mathrm{Cl}_{3} .{ }^{31}$ Due to the necessary high temperatures required to evaporate copper chloride the deposition temperature is restricted to higher substrate temperatures which led to complicated surface chemistry straying from ideal ALD behavior. These ALD studies were carried out on $\mathrm{Al}_{2} \mathrm{O}_{3}{ }^{32}$ and $\mathrm{Ta}^{33}$ substrates using elemental zinc and molecular hydrogen as reducing agents, respectively. Prior to the experiments, the leading concern for depositing copper metal films with zinc was the likely dissolution of zinc into copper forming a brass alloy. Dissolution of zinc occurred and proved problematic due to its slow outgassing from the surface which continued throughout the purging and following copper chloride pulse. ${ }^{32}$ This led to both precursors interacting in the gas phase which caused a large film gradient across the furnace and also high growth rates indicative of a CVD component to film growth. Results from ALD work testing Ta substrates showed that a film of copper metal 30 to $40 \mathrm{~nm}$ could be deposited without $\mathrm{H}_{2}$ pulses due to the reduction of $\mathrm{CuCl}$ by the substrate to form thermodynamically favoured tantalum chloride species (corroborated by XPS analysis). ${ }^{33}$ Beyond the $30-40 \mathrm{~nm}$ of film deposition, the reduction of $\mathrm{CuCl}$ surface species by 
hydrogen was the rate determining step and required an estimated activation energy of 80 $\mathrm{kJ} / \mathrm{mol}$. Saturated growth behavior during the $\mathrm{H}_{2}$ pulse was not achieved even with pulse lengths greater than $20 \mathrm{~s}$.

\subsection{Copper Precursors for Atomic Layer Deposition}

Copper complexes of bidentate ligands have shown greater success as precursors for CVD and ALD. The copper(II) complexes adopt square planar or distorted tetrahedral geometry and the copper(I) complexes are typically dimeric when homoleptic with the copper atom in a two-coordinate, linear geometry. Many copper(I) compounds can be stabilised by Lewis bases as monomers where the bidentate ligand chelates the copper atom in a three or four-coordination environment depending on the Lewis base.

Monomeric copper(I) complexes have been of interest in recent years due to their facile disproportionation reaction to copper(II) and copper metal. ${ }^{34}$ Research efforts were focused on controlling physical properties (eg. melting point, viscosity, solubility) to maximize precursor delivery into a CVD chamber via direct liquid injection and, also, to control the temperature of the disproportionation reaction which is important to obtain the high purity films with resistivities within $10 \%$ of bulk copper. ${ }^{35}$ The precursors that received most of the attention were acetylacetonate (acac) copper(I) complexes stabilized by soft bases such as alkenes and alkynes. ${ }^{36}$ Various substitution patterns of the acac ligand at the 1- and 5-positions with alkyl or fluoro groups were studied and resulted in control of physical properties and also affected the bonding around the copper center. ${ }^{37}$ For example, a higher degree of fluorination of the acac ligand decreased the electron density at the copper center and strengthened the copper-Lewis base bond. Many Lewis bases were studied to increase the bond strength towards copper. ${ }^{36}$ Dissociation of the 
$\mathrm{Cu}(\mathrm{I})-\mathrm{L}$ bond could lead to decomposition of the precursor in its source container or clogging of the direct-liquid-injection instrumentation which was undesirable. ${ }^{35}$ The electron donating ability of the Lewis base could be increased by attaching electron donating groups such as silyl groups ${ }^{38}$ or a conjugated unsaturated bond. ${ }^{36} \mathrm{~A}$ small consequence of varying the substitution of the Lewis base was control over the initial steps leading to precursor chemisorption; ${ }^{37}$ however, ultimately the rate limiting step to copper metal deposition was the surface disproportionation reaction. ${ }^{39}$ The success of these precursors in the CVD of high purity copper films at low temperatures meant that they could not be precursors for ALD.

\subsubsection{Cu KI5}

A series of monomeric copper(I) complexes were prepared and studied to overcome the limited applicability of precursors stabilised by vinyltrimethylsilyl (vtms) to only CVD processes. In these complexes, the Lewis base (i.e. vtms) was tethered to the anionic ligand (Figure 1.1). For this to be feasible the acetylacetonate framework required substitution of at least one of the oxygen atoms to nitrogen, thus changing the acetylacetonate to a ketoiminate or a dikitiminate.

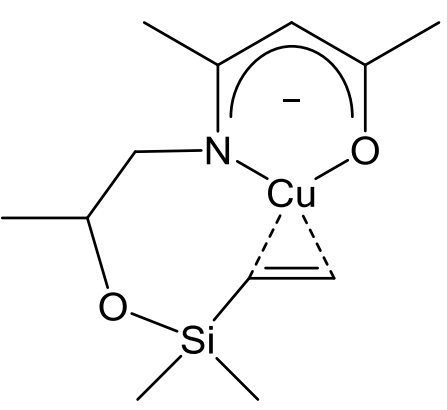

Figure 1.1 The copper ketoiminate precursor CuKI5 with a tethered vinyl-silyl Lewis base. 
The tethered VTMS group coordinated intramolecularly to the copper atom forming a seven-membered ring and prevented thermal loss of the VTMS group inhibiting disproportionation. As a result, these precursors could be heated to higher temperatures generating higher vapour pressure without decomposition. For example, these complexes have vapour pressures above 2 Torr at $140{ }^{\circ} \mathrm{C}$ while the untethered analogues will decompose when heated in attempts to achieve similar vapour pressures. ${ }^{40}$ The initial report on these complexes included experiments demonstrating behavior characteristic of ALD precursors, except no saturation curves were collected. ${ }^{41}$ Growth rates varied between $0.1-0.9 \AA$ /cycle inside a temperature window of $150-200{ }^{\circ} \mathrm{C}$ which possibly indicates a CVD film growth component due to the lack of a steady ALD growth window. Later reports demonstrated promising results for these complexes as CVD precursors in conjunction with formic acid as the reducing agent. ${ }^{42}$ These precursors were not expected to deposit copper via a disproportionation mechanism; therefore, in theory, total precursor consumption was being achieved. Recent surface studies of adsorbed precursor monolayers on a $\mathrm{Cu}(110)$ single-crystal concluded that the tethered VTMS group dissociated from the copper(I) atom upon chemisorption. Additionally, some evidence for a copper(II) ketoiminate species was observed which indicated a small component of disproportionation chemistry occurring for adsorbed CuKI5. ${ }^{43}$

\subsubsection{Bis(tri-n-butylphosphine) Copper(I) acetylacetonate}

An ALD process was achieved from a monomeric $\mathrm{Cu}(\mathrm{I})$ complex employing $\left({ }^{\mathrm{n}} \mathrm{Bu}_{3} \mathrm{P}\right)_{2} \mathrm{Cu}(\mathrm{acac})$ as a precursor. The bis-phosphine formulation is more thermally stable towards disproportionation than their mono-phosphine analogues. ${ }^{44}$ This complex does not possess the high volatility common amongst monomeric copper(I) precursors and has 
a vapour pressure behavior comparable to copper(II) acetylacetonate; however, it is a liquid under standard conditions and can be delivered via direct-liquid-injection. The ALD process was developed for thin films of $\mathrm{Cu}_{2} \mathrm{O}$ and used wet $\mathrm{O}_{2}$ as a co-reactant at temperatures between $100-135^{\circ} \mathrm{C}$ on a variety of substrates $\left(\mathrm{Ta}, \mathrm{Ru}, \mathrm{TaN}, \mathrm{SiO}_{2}\right) .{ }^{45}$ Surprisingly, despite the molecule's saturated coordination sphere it chemisorbed at low temperatures via dissociation of both phosphines. ${ }^{46}$ A narrow ALD window was discovered adjacent to the onset of surface disproportionation and had a growth rate of $0.1 \AA /$ cycle. The $\mathrm{Cu}_{2} \mathrm{O}$ thin films were subsequently reduced under formic acid flow at $110-120^{\circ} \mathrm{C}$ to be used as seed layers for electrochemical copper deposition.

\subsubsection{Copper(II) acetylacetonate precursors}

Many copper(II) complexes have also been studied as viable precursors for ALD. With these precursors, disproportionation is not a concern; however, they tend to require high temperatures or strong reducing agents to obtain metallic films. For example, copper(II) acetylacetonate (Figure 1.2) has been employed in ALD processes using molecular hydrogen and hydrogen radicals at temperatures of $250{ }^{\circ} \mathrm{C}^{47}$ and between 85 $140{ }^{\circ} \mathrm{C},{ }^{48,49}$ respectively. This difference in temperatures highlights how reactivity of chemisorbed $\mathrm{Cu}(\mathrm{II})$ precursors are a more relevant concern rather than precursor adsorption. Surface studies of copper(II) acetylacetonate on $\mathrm{Ni}(110)$ and $\mathrm{Cu}(110)$ single crystals concluded that reduction of the precursor ion occurs for adsorbed molecules near room temperature and that the role of the second precursor is to remove the remaining organic molecules. $^{50}$ 


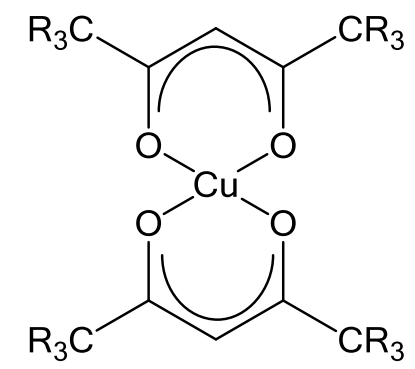

Figure 1.2 Copper(II) acetylacetonate, $\mathrm{R}=\mathrm{H}$; tetramethylheptanedionate, $\mathrm{R}=\mathrm{Me}$; hexafluoroacetylacetonate, $\mathrm{R}=\mathrm{F}$.

Two variants of copper(II) acetylacetonate have been studied in ALD processes. Copper(II) hexafluoroacetylacetonate (hfac) and copper(II) tetramethylheptanedionate (tmhd) are advantageous due to higher volatility ${ }^{51}$ and higher thermal stability, respectively (Figure 1.2). A wide deposition temperature window is possible for copper(II) hfac due to a low sublimation temperature. Alcohols and formaldehyde were used as reducing agents at temperatures of $230-300{ }^{\circ} \mathrm{C}$ showing a high growth rate of 2.2 $\AA /$ cycle, for which, the self-limiting nature was not confirmed. ${ }^{52}$ These high temperatures were necessary due to the choice of reducing agents. Half cycles for film nucleation at considerably lower temperatures is feasible. Stable monolayers of copper(II) hfac adsorbed onto $\mathrm{Al}_{2} \mathrm{O}_{3}$ have been studied at substrate temperatures of $80{ }^{\circ} \mathrm{C} .{ }^{53}$ Hydrogen plasma was used to complete the full ALD cycle. Additonally, room temperature reduction of adsorbed copper(II) hfac on TiN substrates by molecular hydrogen catalysed by pyridine has been demonstrated. ${ }^{54}$ The growth rate from $25-100{ }^{\circ} \mathrm{C}$ was $0.18 \AA /$ cycle and the films contained significant oxygen and carbon impurities.

ALD work based on copper(II) thmd have focused on the selective deposition of copper metal on Pd or Pt seed layers using molecular hydrogen at substrates temperatures of $135-260{ }^{\circ} \mathrm{C} .{ }^{55}$ The initial surface mechanism was believed to involve $\mathrm{Pd} / \mathrm{Pt}$ catalysed 
reduction of the copper(II) tmhd monolayer during the hydrogen pulse; however, recently it has been shown that adsorption of copper(II) tmhd is readily reversible during the purging steps of the ALD cycle. ${ }^{56}$ The surface interaction of the two precursors occurs because the Pd seed layer retains dissociated hydrogen until the following copper(II) tmhd pulse. Intermixing of the $\mathrm{Cu}$ layer with the Pd seed layers also occurred and led to a surface presence of Pd which could continually participate in the uptake of hydrogen as the copper film accumulated.${ }^{57}$ Reversible adsorption of copper(II) thmd was not a concern when metal nitrides, metal oxides or metals with low activity towards hydrogen are used as substrates since ALD using hydrogen plasma as a co-reactant has been demonstrated on $\mathrm{SiO}_{2}, \mathrm{Au}$, and $\mathrm{TaN}_{\mathrm{x}}$ between 90 to $250{ }^{\circ} \mathrm{C} .{ }^{58}$

\subsubsection{Copper(II) diketoiminate precursors}

Copper(II) ketoiminates are compelling alternatives to the diketonates. The lower oxygen content of the ketoiminate ligand is advantageous for metal depositions and the variability of the $\mathrm{N}$-substituent can be used to tune the physical characteristics of the precursor and process conditions. ${ }^{59}$ Due to the added steric bulk of the N-substituents the solid state structures of copper(II) ketoiminates adopt distorted square planar or tetrahedral geometry. ${ }^{60}$ The diketonates are flat, square planar molecules that efficiently stack in the solid state. Consequently, copper(II) ketoiminates have higher volatility and

Figure 1.3 Copper(II) 2-ethylimino-4-pentanonate

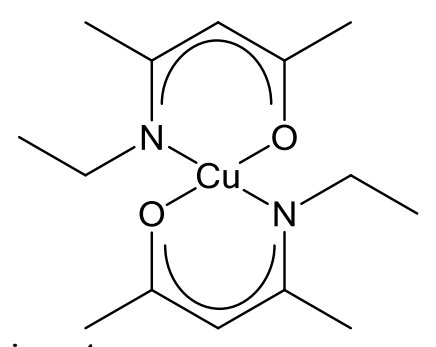


low melting points as a result of the disrupted intermolecular interaction. ${ }^{61}$ Copper metal films have been deposited employing copper(II) 4-ethyimino-pentan-2-one (Figure 1.3) and molecular hydrogen as a co-reactant at deposition temperatures between $120-220^{\circ} \mathrm{C}$ on Pt and Ru substrates. ${ }^{62}$ Growth rates of $1.2-1.7 \AA$ /cycle were reported without data confirming self-limited growth. The main focus was the sheet resistance of the copper films. Sheet resistance was found to be dependent on deposition temperature due to changes in film morphology. Plasma enhanced ALD was also possible within a temperature window of $30-100{ }^{\circ} \mathrm{C} .{ }^{61}$ Even though the precursor was evaporated at 85 ${ }^{\circ} \mathrm{C}$ the growth rates and precursor pulse length did not indicate precursor transport or condensation to be a concern. Self-limited growth was confirmed and a growth rate of 0.3 $\AA$ /cycle was observed. With preferentially oriented $\mathrm{Ru}(001)$ thin film substrates, smooth and continuous $\mathrm{Cu}(111)$ oriented films are obtained which are desirable features for seed layers in the electrodeposition of copper. ${ }^{63}$ Evidently, complete in-filling of high aspect ratio trenches was demonstrated for a $13 \mathrm{~nm} \mathrm{Cu}$ seed layer using the low-temperature plasma enhanced process. ${ }^{61}$

\subsubsection{Copper(I) and (II) dikitiminate precursors}

Substitution of both oxygen atoms for nitrogen of the diketonate ligand affords the diketiminate ligand for which copper(I) and (II) complexes have been studied as
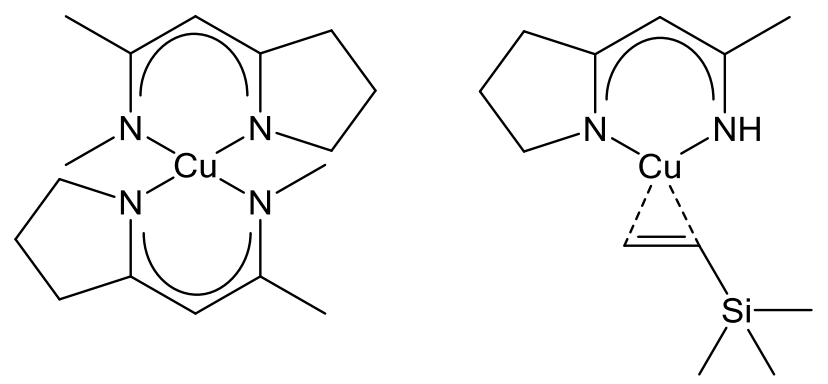

Figure 1.4 Asymmetric copper(I) and (II) dikitiminate precursors. 
precursors (Figure 1.4). ${ }^{64,65}$ The ligand framework is highly amenable to symmetric and unsymmetric variation of four alkyl groups including cyclization of a nitrogen atom to the ligand backbone. In comparison to the diketonate and ketoiminate copper complexes, the diketiminates are more volatile which can be attributed to their distorted tetrahedral geometries, dissymmetry and alkyl substitutions. ${ }^{64-66}$ Pulsed CVD was demonstrated for a monomeric copper(I) diketiminate at deposition temperatures of $120^{\circ} \mathrm{C}$ which required a silane as a co-reactant to achieve films with low levels of contaminants. ${ }^{67}$ Deposition temperatures could be lowered to $45^{\circ} \mathrm{C}$ if pyrazole was adsorbed to the surface prior to passing the copper precursor over the substrate; however, a self-limiting growth behavior was not confirmed and film thickness was dependent on precursor pulse. ${ }^{68}$ There is limited data regarding use of a copper(II) diketiminate precursors to grow copper thin films. Considering their superior volatility, a low temperature plasma enhanced process similar to the reports for copper(II) diketoiminates would be promising.

\subsubsection{Copper(I) amidinate precursors}

Copper(I) amidinate complexes are a unique class of copper ALD precursor that do not undergo the detrimental copper(I) disproportionation reaction. ${ }^{69}$ These complexes share similarities to copper(I) amide complexes; however possess desirable properties of thermal stability and volatility. Like the copper(I) amides, the copper(I) amidinates are

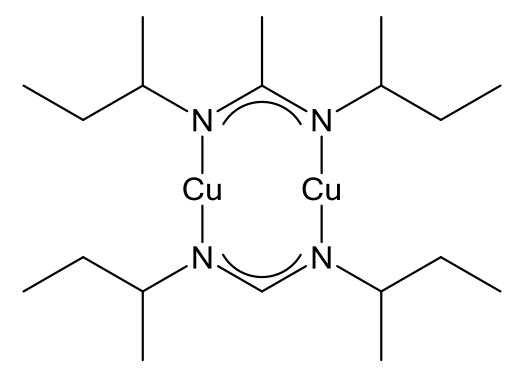

Figure 1.5 Copper(I)- $N, N^{\prime}$-di-sec-butylacetamidinate 
nitrogen donor ligands with linear coordination geometry around each copper center (Figure 1.5). They are only stable in the copper(I) oxidation state. Typically, a dimeric structure is obtained with a highly symmetric metallocycle containing approximately equivalent $\mathrm{Cu}-\mathrm{N}$ and $\mathrm{C}-\mathrm{N}$ bond lengths due to the resonant imine bond of the amidinate ligand. The amidinate ligands provide a high degree of steric protection of the coordinated copper centers using bulky aliphatic groups that allow only weak intermolecular interactions. Along with the strong bond between copper and nitrogen, these complexes generate appreciable vapour pressures when heated. Copper metal films have been deposited on a variety of substrates at deposition temperature of $150-190^{\circ} \mathrm{C}$ using molecular hydrogen as a co-reactant. ${ }^{70}$ Oxynitride,${ }^{71}$ nitride,${ }^{72}$ and sulphide ${ }^{73}$ films can be deposited at temperatures above $130^{\circ} \mathrm{C}$ with self-limited film growth. Surface studies on silica surfaces have shown chemisorption proceeding through displacement of one amidinate ligand by a surface hydroxyl group. ${ }^{74}$ The remaining surface amidinates are removed following the hydrogen pulse. On metallic surfaces, a monolayer of chemisorbed precursor exists as reduced copper atoms and dehydrogenated ligand fragments. ${ }^{75}$ The primary role of the hydrogen pulse is to desorb any ligand fragments and not to reduce the deposited copper atom. The processes developed for copper(I) amidinate complexes provide suitable seed layers ${ }^{76}$ and impressive means to completely in-fill narrow trench features employing a surface activation agent. ${ }^{77}$

\subsubsection{Copper(II) aminoalkoxide precursors}

Copper(II) aminoalkoxides were developed to fill the void of copper alkoxides in copper precursors. These monomeric precursors have square planar geometry stabilised 
by an amino group tethered to each alkoxide (Figure 1.6). ${ }^{78}$ Similar to the simple copper(II) alkoxides,

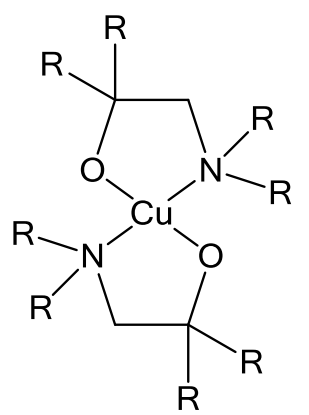

Figure 1.6 Copper(II) aminoalkoxides, $\mathrm{R}=\mathrm{H}$, alkyl, fluoroalkyl.

the aminoalkoxides can undergo thermal decomposition via $\beta$ or $\gamma$-hydride abstraction and afford pure copper metal films above $200{ }^{\circ} \mathrm{C}$ in CVD processes. ${ }^{79,80}$ Fortunately, these complexes show high volatility even without fluorinated ligands ${ }^{81}$ and several low temperature ALD processes have been developed. A thermal ALD study employing molecular hydrogen as the co-reactant on Ru substrates found low film nucleation and growth rates within the viable temperature window of the precursor. ${ }^{82}$ In the same work, copper seed layers were more easily fabricated through a thermal copper nitride ALD process between $100-140{ }^{\circ} \mathrm{C}$ followed by reduction at $200{ }^{\circ} \mathrm{C}$. Plasma enhanced ALD has been reported for two copper(II) aminoalkoxide precursors. ${ }^{63,83}$ Deposition temperatures down to $30{ }^{\circ} \mathrm{C}$ and up to the decomposition temperature of the specific copper(II) aminoalkoxide (i.e. $>160^{\circ} \mathrm{C}$ ) were studied and growth rates were measured to be $0.2-$ $0.6 \AA /$ cycle. The copper metal films deposited at low temperatures are smooth and consist of dense, connected particles with a crystalline texture strongly influenced by that of the substrate. ${ }^{63}$ Film impurities increased for films deposited at low substrate temperatures due to inefficient desorption of ligand fragments which resulted in film resistivity higher than bulk copper. Copper(II) aminoalkoxide complexes have also been 
studied in combination with unconventional co-reactants to achieve low-temperature copper deposition without plasma generated atomic hydrogen. Volatile alkylation reagents are particularly useful for copper metal ALD due to facile ligand exchange reactions and the instability of copper alkyl complexes. ${ }^{84}$ For example, copper(II) dimethylamino-2-propoxide and diethyl zinc were used as co-reactants to deposit copper metal films between $100-120^{\circ} \mathrm{C}$ at a self-limiting growth rate of $0.2 \AA /$ cycle. ${ }^{85}$ The ALD temperature window was narrow in order to limit film impurities. Lower temperatures decreased desorption of the $\mathrm{Zn}$ products resulting from the ligand exchange surface reactions and high temperatures caused decomposition of the diethyl zinc co-reactant. Strong reducing agents such as hydrazine ${ }^{86}$ or amino boranes ${ }^{87}$ are also suitable coreactants capable of copper metal ALD near $100{ }^{\circ} \mathrm{C}$. These reducing agents have optimal performance when used along with formic acid and/or a metal catalyst to assist the reduction chemistry.

\subsubsection{Copper Precursor Summary}

The shortcomings of alkyl, amido and alkoxide homoleptic copper compounds is mitigated by a vast library of suitable chelation complexes available for copper CVD and ALD processes. Very few examples of copper(I) precursors are available for ALD because the minimum temperature for disproportionation reactions precedes the minimum adsorption temperature. As a general guideline, if copper(I) and (II) oxidation states exist for a given ligand class then only the copper(II) compounds will be useful ALD precursors. Copper(I) amidinates are, so far, the best preforming copper(I) precursor. The ALD window for copper metal films has been limited by the activity of molecular hydrogen. Plasma-enhanced ALD or thermal ALD with a novel co-reactant 
may yield low temperature results for the amidinate precursors. The evolution of the copper(II) precursor has traversed step-by-step from chelating oxygen ligands forming six membered metallo-cycles to chelating nitrogen ligands forming five-membered metallo-cycles. The progression was fueled by improvements in volatility due to, in part, changes in coordination geometry and was accompanied by losses in thermal stability. ${ }^{88}$ Copper metal deposition by thermal ALD from copper(II) precursors has been reported between $100-250{ }^{\circ} \mathrm{C}$. The lower temperature limit depends on the activity of molecular hydrogen with the substrate and the upper limit depends on the stability of the precursor. Novel co-reactants have widened the ALD window by lowering the minimum deposition temperature. Plasma-enhanced processes seem to have bottomless windows given the precursor is sufficiently volatile. Processes and formulations for new copper (I) and (II) precursors will continue to be explored. For example, additional ALD studies for copper(II) pyrrolylaldiminates are expected to be reported.$^{84}$ Also, heteroleptic copper(II) compounds have recently appeared in the academic ${ }^{89}$ and patent ${ }^{90}$ literature. ALD studies should be forthcoming.

\subsection{Upcoming Thesis Chapters}

This thesis can be divided into two parts about separate research programs dealing with developing copper precursors. The first begins with a ligand modification to amidinates and proceeds through numerous thermolysis studies ending with an improved ligand design and thermally robust precursors for group 11 metals. Briefly, Chapter 2 describes the synthesis and thermal characterisation of copper(I) guanidinate compounds followed by their use as precursors for copper metal films without requiring a reducing agent. Comparative experiments for a copper(I) amidinate were performed and the 
differences are discussed with respect to a particular decomposition mechanism, CDI deinsertion. Chapter 3 involves an investigation with advanced techniques to determine $\beta$ hydride abstraction as the gas phase thermal decomposition mechanism of a copper(I) guanidinate compound. Previously, iminopyrrolidinate ligands were developed in our group to eliminate CDI-deinsertion as a mechanism of decomposition. In Chapter 4, a modified iminopyrrolidine which also prevents $\beta$-hydride abstraction is described. Chapter 4 and 5 details a comparison of several copper(I) iminopyrrolidinates, as well as, silver(I) and gold(I) iminopyrrolidinates to highlight the improved ligand design.

The second part of this thesis introduces diamino carbenes (e.g. NHC) to the library of ligands available for designing copper precursors. Motivation came from the lack of monomeric copper(I) amidinate, guanidinate, and iminopyrrolidinate complexes in the literature. Stable carbenes were used to break their dimeric structure; however, the monomers were less volatile and less stable. Chapter 6 entails two monomeric copper(I) amide precursors employing two different NHCs and their use in PE-ALD of copper metal films. The differences in their ALD chemistry could only be attributed to the differences in the NHCs. Chapter 7 explores a large series of cyclic and acyclic diamino carbenes and how their differences affect precursor properties.

\subsection{References}

12013 Edition of the International Technology Roadmap for Semiconductors; http://www.itrs.net/Links/2013ITRS/2013Chapters/2013Interconnect_Summary.pdf.

2 Havemann, R. H.; Hutchby, J. A. Proceedings of the IEEE 2001, 89, 586 - 601.

3 Moore, G. E. Electronics, 1965, 114-117. 
4 Theis, T. N. IBM J. Res. Develop. 2000, 44, 379-390.

5 Gambino, J.; Chen, F.; He, J. Custom Integrated Circuits Conference 2009, 141, 13 16.

6 Andricacos, P. C.; Uzoh, C.; Dukovic, J. O.; Horkans, J.; Deligianni, H. IBM J. Res. Develop. 1998, 42, 567-574.

7 Merchant, S. M.; Kang, S. H.; Sanganeria, M.; Schravendijk, B. v.; Mountsier, T. JOM 2001, 53, 43-48.

8 Gambino, J. P. ECS Transactions 2011, 35, 687-699.

9 Kaloyeros, A. E.; Eisenbraun, E. Annu. Rev. Mater. Sci. 2000, 30, 363-385.

10 Wojcik, H.; Friedmann, M.; Feustel, F.; Albert, M.; Ohsiek, S.; Metzger, J.; Voss, J.; Bartha, J. W.; Wenzel, C. IEEE Int. Interconnect Techology Conf. 2007, 19-21.

11 Yang, C.-C.; Spooner, T.; Ponoth, S.; Chanda, K.; Simon, A.; Lavoie, C.; Lane, M.; Hu, C.-K.; Liniger, E.; Gignac, L.; Shaw, T.; Cohen, S.; McFeely, F.; Edelstein, D. IEEE Int. Interconnect Techology Conf. 2006, 187-189.

12 George, S. M. Chem. Rev. 2010, 110, 111-131.

13 Li, W.-M. Chem. Vap. Dep. 2013, 19, 82-103.

14 Miikkulainen, V.; Leskela, M.; Ritala, M.; Puurunen, R. L. J. Appl. Phys. 2013, 113, 021301.

15 Bertz S. H.; Dabbagh, G. Tetrahedron 1989, 45, 425-434.

16 Wada, K.; Tamura, M.; Kochi, J. J. Am. Chem. Soc. 1970, 92, 6656-6658.

17 Thiele, K.-H.; Kohler, J. J. Organometallic Chem. 1968, 12, 225-229.

18 Whitesides, G. M.; Panek, E. J.; Stedronsky, E. R. J. Am. Chem. Soc. 1972, 94, 232239.

19 Whitesides, G. M.; Stedronsky, E. R.; Casey, C. P.; Filippo, J. S. J. Am. Soc. Chem. 1970, 92, 1426-1427.

20 Hope, H.; Power, P. P. Inorg. Chem. 1984, 23, 936-937. 
21 Gambarotta, S.; Bracci, M.; Floriani, C.; Chiesi-Villa, A.; Guastini, C. J. Chem. Soc. Dalton. Trans. 1987, 1883-1888.

22 James, A. M.; Laxman, R. K.; Fronczek, F. R.; Macerick, A. W. Inorg. Chem. 1998, 37, 3785-3791.

23 Tsuda, T.; Watanabe, K.; Miyata, K.; Yamamoto, H.; Saegusa, T. Inorg. Chem. 1981, 20, 2728-2730.

24 Whitesides, G. M.; Sadowski, J. S.; Lilburn, J. J. Am. Chem. Soc. 1974, 96, 28292835 .

25 Costa, G.; Camus, A.; Marsich, N. J. Inorg. Nucl. Chem. 1965, 27, 281-285.

26 Tsuda, T.; Toshiaki, H.; Saegusa, T. J. Am. Chem. Soc. 1972, 94, 658-659.

27 Brubaker, C. H.; Wicholas, M. J. Inorg. Nucl. Chem. 1965, 27, 59-62.

28 Singh, J. V.; Baranwal, B. P.; Mehrotra, R. C. Z. anorg. allg. Chem. 1981, 477, 235240 .

29 Nelson, K. J.; Guzei, I. A.; Lund, G. S.; McGaff, R. W. Polyhedron 2002, 21, 2017 2020.

30 Jeffries, P.; Girolami, G. S. Chem. Maters. 1989, 1, 8-10.

31 Brewer, L.; Lofgren, N. L. J. Am. Chem. Soc. 1950, 72, 3038.

32 Juppo, M.; Ritala, M.; Leskelä, M. J. Vac. Sci. Technol. A 1997, 15, 2330-2333.

33 Mårtensson, P.; Carlsson, J.-O. Chem. Vap. Dep. 1997, 3, 45-50.

34 Hampden-Smith, M. J.; Kodas, T. T. Polyhedron 1995, 14, 699-732.

35 Petersen, G. A.; Parmeter, J. E.; Apblett, C. A.; Gonzales, M. F.; Smith, P. M. Omstead, T. R.; Norman, J. A. T. J. Electrochem. Soc. 1995, 142, 939-944.

36 Doppelt, P. Coord. Chem. Rev. 1998, 178-180, 1785-1809.

37 Chen, T.-Y.; Vaissermann, J.; Ruiz, E.; Senateur, J. P.; Doppelt, P. Chem. Mater. 2001, 13, 3993-4004.

38 Tran, P. D.; Doppelt, P. Surf. Coat. Technol. 2007, 201, 9066-9070. 
39 Girolami, G. S.; Jeffries, P. M.; Dubois, L. H. J. Am. Chem. Soc. 1993, 115, 10151024.

40 Norman, J. A. T.; Perez, M.; Schulz, S. E.; Waechtler, T. Microelectronic Engineering 2008, 85, 2159-2163.

41 Norman, J. A. T.; Perez, M.; Lei, X.; Cheng, H. ECS Transactions 2007, 3, 161-170.

42 Song, H.; Norman, J. A. T.; Shimogaki, Y. Microelectronic Engineering 2010, 87, 249-253.

43 Ma, Qiang, Zaera, F. J. Vac. Sci. Technol. A 2015, 33, DOI: 10.1116/1.4896940

44 (a) Shin, H.-K.; Hampden-Smith, M. J.; Duesler, E. N.; Kodas, T. T. Polyhedron, 1991, 10, 645-647, (b) Shin, H.-K.; Hampden-Smith, M. J.; Duesler, E. N.; Kodas, T. T. Can. J. Chem. 1992, 70, 2954-2966.

45 Waechtler, T.; Ding, S.-F.; Hofmann, L.; Mothes, R.; Xie, Q.; Oswald, S.; Detavernier, C.; Schulz, S. E.; Qu, X.-P.; Lang, H.; Gessner, T. Microelectronic Engineering 2011, 88, 684-689.

46 Dhakal, D.; Waechtler, T.; Schulz, S. E.; Gessner, T.; Lang, H.; Mothes, R.; Tuchscherer, A. J. Vac. Sci. Technol. A 2014, 32, 041505.

47 Utriainen, M.; Kroger-Laukkanen, M.; Johansson, L.-S.; Niinisto, L. Appl. Surf. Sci.2000, 157, 151-158.

48 Niskanen, A.; Rahtu, A.; Sajavaara, T.; Arstila, K.; Ritala, M.; Leskela, M. J. Electrochem. Soc. 2005, 152, G25-G28.

49 Wu, L.; Eisenbraun, E. J. Vac. Soc. Technol. B 2007, 25, 2581-2585.

50 Qiang, M.; Zaera, F. J. Vac. Sci. Technol. A 2013, 31, $01 \mathrm{~A} 112$.

51 Fahlman, B. D.; Barron, A. R. Adv. Mater. Opt. Electron. 2000, 10, 223-232.

52 Solanki, R.; Pathangey, B. Electrochem. Solid-State Lett. 2000, 3, 479-480.

53 Chaukulkar, R. P.; Thissen, N. F. W.; Rai, V. R.; Agarwal, S. J. Vac. Sci. Technol. A 2014, 32, $01 \mathrm{~A} 108$.

54 Kang, S.-W.; Yun, J.-Y.; Chang, Y. H. Chem. Mater. 2010, 22, 1607-1609.

55 Martensson, P.; Carlsson, J.-O. J. Electrochem. Soc. 1998, 145, 2926-2931. 
56 Jiang, X.; Wang, H,; Qi, J.; Willis, B. G. J. Vac. Sci. Technol. A. 2014, 32, 041513.

57 Hsu, I. J.; McCandless, B. E.; Weiland, C.; Willis, B. G. J. Vac. Sci. Technol. 2009, 27, 660-667.

58 Jezewski, C.; Lanford, W. A.; Wiegand, C. J.; Singh, J. P.; Wang, P.-I.; Senkevich, J. J.; Lu, T.-M. J. Electrochem. Soc. 2005, 152, C60-C64.

59 Stabnikov, P. A. Russ. J. Gen. Chem. 2013, 83, 1919-1927.

60 Baidina, I. A.; Stabnikov, P. A.; Vasiliev, A. D.; Gromilov, S. A.; Igumenov, I. K. J. Struct. Chem. 2004, 45, 671-677.

61 (a) Mao, J.; Eisenbraun, E.; Omarjee, V.; Korolev, A.; Dussarrat, C. ECS Transactions 2011, 35, 125-132, (b) Mao, J.; Eisenbraun, E.; Omarjee, V.; Korolev, A.; Dussarrat, C. IEEE Trans. Semicond. Manuf. 2013, 26, 17-22.

62 (a) Park, K.-M.; Kim, J.-K.; Han, B.; Lee, W.-J.; Kim, J.; Shin, H.-K. Microelectronic Engineering 2012, 89, 27-30, (b) Han, B.; Park, K.-M.; Park, K.; Park, J.-W.; Lee, W.-J. IEEE Int. Interconnect Techology Conf.2009, 173-174.

63 Hagen, D. J.; Connolly, J.; Nagle, R.; Povey, I. M.; Rushworth, S.; Carolan, P.; Ma, P.; Pemble, M. E. Surf. Coat. Technol. 2013, 230, 3-12.

64 Park, K.-H.; Bradley, A. Z.; Thompson, J. S.; Marshall, W. J. Inorg. Chem. 2006, 45, 8480-8482.

65 Park, K.-H.; Marshall, W. J. J. Am. Soc. Chem. 2005, 127, 9330-9331.

66 Morozo, N. B.; Stabinokov, P. A.; Baidina, I. A.; Semyannikov, P. P.; Trubin, S. V.; Igumenov, I. K. J. Struct. Chem. 2007, 48, 889-898.

67 Thompson, J. S.; Zhang, L.; Wyre, J. P.; Brill, D. J.; Lloyd, K. G. Thin Solid Films 2009, 517, 2845-2850.

68 Thompson, J. S.; Zhang, L.; Wyre, J. P.; Brill, D.; Li, Z. Organometallics 2012, 31, 7884-7892.

69 Li, Z.; Barry, S. T.; Gordon, R. G. Inorg. Chem. 2005, 44, 1728-1735.

70 Li, Z.; Rahtu, A.; Gordon, R. G. J. Electrochem. Soc. 2006, 153, C787-C794. 
71 Kim, H.; Bhandari, H. B.; Xu, S.; Gordon, R. G. J. Electrochem. Soc. 2008, 155, H496-H503.

72 Li, Z.; Gordon, R. G. Chem. Vap. Dep. 2006, 12, 435-441.

73 Martinson, A. B. F.; Elam, J. W.; Pellin, M. J. Appl. Phys. Lett. 2009, 94, 123107.

74 Dai, M.; Kwon, J.; Halls, M. D.; Gordon, R. G.; Chabal, Y. J. Langmuir 2010, 26, 3911-3917.

75 (a) Ma, Q.; Guo, H.; Gordon, R. G.; Zaera, F. Chem. Mater. 2010, 22, 352-359, (b) Ma, Q.; Guo, H.; Gordon, R. G.; Zaera, F. Chem. Mater. 2011, 23, 3325-3334, (c) Ma, Q.; Zaera, F.; Gordon, R. G. J. Vac. Soc. A 2012, 30, $01 \mathrm{~A} 114$.

76 Li, Z.; Gordon, R. G.; Farmer, D. B.; Lin, Y. Vlassak, J. Electrochem. Solid-State Lett. 2005, 8, G182-G185.

77 Au, Y.; Lin, Y.; Gordon, R. G. J. Electrochem. Soc. 2011, 158, D248-D253.

78 Goel, S. C.; Kramer, K. S.; Chiang, M. Y.; Buhro, W. E. Polyhedron 1990, 9, 611613.

79 Becker, R.; Devi, A.; Wei, J.; Weckenmann, U.; Winter, M.; Keiner, C.; Becker, H.W.; Fischer, R. A. Chem. Vap. Dep. 2003, 9, 149-156.

80 Park, J. W.; Jang, H. S.; Kim, M.; Sung, K.; Lee, S. S.; Chung, T.-M.; Koo, S.; Kim, C. G.; Kim, Y. Inorg. Chem. Commun. 2004, 7, 463-466.

81 Chi, Y.; Hsu, P.-F.; Liu, C.-S.; Ching, W.-L.; Chou, T.-Y.; Carty, A. J.; Peng, S.-M.; Lee, G.-H.; Chuang, S.-H. J. Mater. Chem. 2002, 12, 3541-3550.

82 Park, J.-M.; Jin, K.; Han, B.; Kim, M. J.; Jung, J.; Kim, J. J.; Lee, W.-J. Thin Solid Films 2014, 556, 434-439.

83 Moon, D.-Y.; Han, D.-S.; Shin, S.-Y.; Park, J.-W.; Kim, B. M.; Kim, J. H. Thin Solid Films 2011, 519, 3636-3640.

84 (a) Vidjayacooumar, B.; Emslie, D. J. H.; Clendenning, S. B.; Blackwell, J. M.; Britten, J. F.; Rheingold, A. Chem. Mater. 2010, 22, 4844-4853, (b) Vidjayacoumar, B.; Emslie, D. J. H.; Blackwell, J. M.; Clendenning, S. B.; Britten, J. F. Chem. Mater. 2010, 22, 4854-4866. 
85 Lee, B. H.; Hwang, J. K.; Nam, J. W.; Lee, S. U.; Kim, J. T.; Koo, S. -M.;

Baunemann, A.; Fischer, R. A. Sung, M. M. Angew. Chem. Int. Ed. 2009, 48, 45364539.

86 Knisley, T. J.; Ariyasena, T. C.; Sajavaara, T.; Saly, M. J.; Winter, C. H. Chem. Mater. 2011, 23, 4417-4419.

87 Kalutarage, L. C.; Clendenning, S. B.; Winter, C. H. Chem. Mater. 2014, 26, 37313738.

88 (a) Patterson, G. S.; Holm, R. H. Bioinorganic Chemistry 1975, 4, 257-275, Yokoi, H.; Addison, A. W. Inorg. Chem. 1977, 16, 1341-1349.

89 (a ) Krisyuk, V. V.; Sysoev, S. V.; Rumyantsev, Y. M.; Prokhorova, S. A.; Maximovskiy, E. V.; Kosinova, M. L.; Igumenov, I. K. Physics Procedia 2013, 46, 174-182, (b) Krisyuk, V. V.; Baidina, I. A.; Korolkov, I. V.; Semyannikov, P. P.; Stabnikov, P. A.; Trubin, S. V.; Turgambaeva, A. E. Polyhedra 2013, 49, 1-6, (c) Rajendran, N. M.; Maheswari, K.; Reddy, D. Polyhedron 2014, 81, 329-334, (d) Melzer, M. M.; Mossin, S.; Dai, X.; Bartell, A. M.; Kapoor, P.; Meyer, K.; Warren, T. H. Angew. Chem. Int. Ed. 2010, 49, 904-907.

90 Korolev, A. V.; Lansalot-Matras, C. "Heteroleptic Pyrrolecabaldimine Precursors" U.S. Patent 8,431,719, Apr. 30, 2013. 


\section{Chapter: Synthesis and Thermal Chemistry of Copper (I) Guanidinates}

\section{Modified from original manuscript published as:}

Coyle, J. P. '; Monillas, W. H. ${ }^{2}$; Yap, G. P. A. ${ }^{2}$; Barry, S. T. "'Synthesis and Thermal Chemistry of Copper(I) Guanidinates" Inorg. Chem. 2008, 47, 683-689

${ }^{1}$ Department of Chemistry, Carleton University, 1125 Colonel By Drive, Ottawa, Ontario, Canada K1S 5B6.

${ }^{2}$ Department of Chemistry and Biochemistry, University of Delaware, Newark, Delaware 19716.

\subsection{Abstract}

Copper (I) guanidinate dimers were generated by a salt metathesis route, and structurally characterised. The guanidinates differed from the known amidinate dimers because of a large torsion of the dimer ring. This had a direct effect on their thermal chemistry. Thermal reactivity was investigated by several methods, including a novel temperature-resolved, gas-phase method that was monitored by mass spectrometry. The copper guanidinates underwent carbodiimide deinsertion to produce copper metal at temperatures between $225-250{ }^{\circ} \mathrm{C}$ in the gas phase, and at $125^{\circ} \mathrm{C}$ in solution. The amidinate investigated also showed copper deposition at $190{ }^{\circ} \mathrm{C}$ in the gas phase, and 135 ${ }^{\circ} \mathrm{C}$ in solution, but without carbodiimide deinsertion. The guanidinate compounds deposited crystalline copper at $225^{\circ} \mathrm{C}$ in a simple CVD experiment.

\subsection{Introduction}

Thin copper films are important to the advancement of microelectronic devices, and both chemical vapour deposition (CVD) ${ }^{1}$ and atomic layer deposition (ALD) ${ }^{2}$ are methods predicted to be used for copper interconnect production by the International Technology Roadmap for Semiconductors. ${ }^{3}$ Organometallic copper precursors have been 
studied intensely as vaporous precursors for thin metal film deposition by ALD for this reason. ${ }^{4}$ Among copper precursors, copper (I) compounds employing chelate ligands have shown tremendous utility in both CVD and ALD applications. Examples of these compounds include copper (I) and (II) $\beta$-diketonates, ${ }^{5}$ as well as copper (I) ketoiminates, ${ }^{6}$ and $\beta$-diketimidates. ${ }^{7}$

Recently, copper (I) amidinate dimers have been reported as ALD precursors, ${ }^{8}$ as well as to investigate spin delocalization between copper metal centres. ${ }^{9}$ These species exist as dimers wherein the amidinate ligand bridges two copper centres. We have extended this family of copper compounds to include copper(I) guanidinates. The exocyclic amido moiety will change the bonding mode of the ligand by permitting an additional resonance structure to exist for the compound (Figure 2.1). When the ligand has an alkyl in the exocyclic position (Figure 2.1a), the $\pi$ system is restricted to the dimer ring. When the exocyclic position is occupied by an amide, the $\pi$ system can extend to the exocyclic nitrogen, permitting the "iminium" resonance structure C (Figure 2.1b). We were interested if this change in resonance, and its concomitant change in bonding within the dimer ring, would affect the thermal chemistry of the precursor.

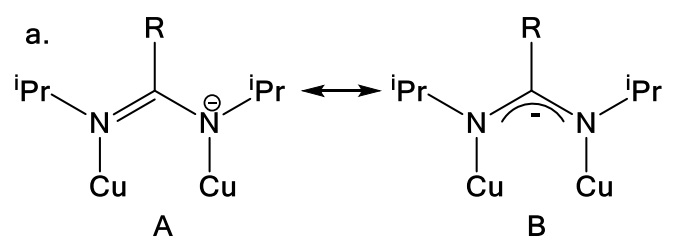

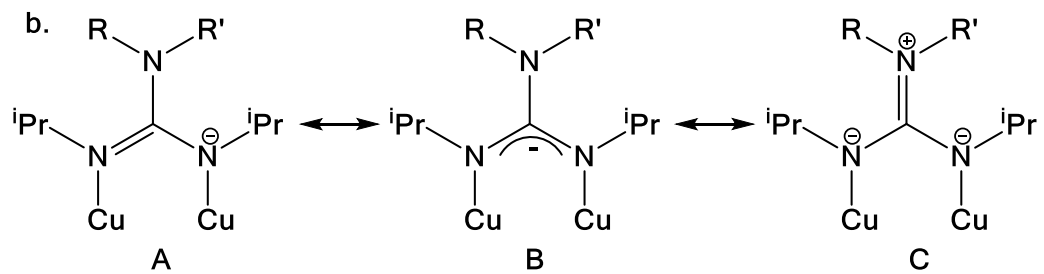

Figure 2.1 Resonance Structures for Copper Amidinate and Guanidinate Ligands. 
Specifically, our interest in copper guanidinate thermal chemistry is to determine whether carbodiimide deinsertion plays a role in their decomposition, as has been demonstrated in aluminum and gallium guanidinates (Figure 2.2). ${ }^{10,11,12}$

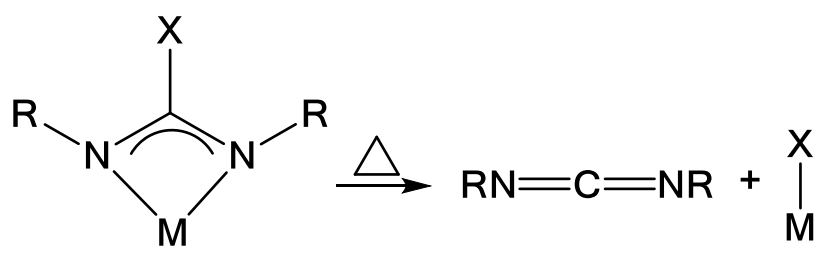

Figure 2.2 Thermally Induced Carbodiimide Deinsertion.

We have synthesized and structurally characterized two copper guanidinates, and investigated their thermal reactivity through a number of experiments. Most significantly, we have adapted a quadropole mass spectrometer to sample a thermolysis apparatus as the precursor vapour is passed through (see Experimental Procedures). By coupling the time-resolved sampling capacity of the spectrometer with the temperature ramp of the furnace, we are able to extract a temperature-resolved mass spectrum of vapour phase species. This experiment allows us to explore the thermal behaviour of the precursors under the conditions they experience during a typical vapour deposition experiment. We have also studied the thermolysis of the known amidinate $\left[\mathrm{MeC}\left({ }^{\mathrm{i}} \mathrm{PrN}\right)_{2} \mathrm{Cu}\right]_{2},{ }^{8 \mathrm{~d}}$ in order to compare thermolysis between amidinates and guanidinates for copper. Finally, the new guanidinate compounds are shown to deposit crystalline copper films at $225^{\circ} \mathrm{C}$ in a simple CVD experiment.

\subsection{Results and Discussion}

To determine the role of carbodiimide deinsertion on copper-containing systems, we have synthesized and studied two copper (I) guanidinates: $\left[\mathrm{Me}_{2} \mathrm{NC}\left({ }^{i} \mathrm{PrN}\right)_{2} \mathrm{Cu}\right]_{2}(\mathbf{1})$ 
and $\left[{ }^{i} \operatorname{PrN}(\mathrm{H}) \mathrm{C}\left({ }^{i} \mathrm{PrN}\right)_{2} \mathrm{Cu}\right]_{2}(2)$. Both of these compounds were made at room temperature by salt metathesis:

$$
\mathrm{RC}\left({ }^{i} \mathrm{PrN}\right)_{2} \mathrm{Li}+\mathrm{CuCl} \rightarrow 1 / 2\left[\mathrm{RC}\left({ }^{i} \mathrm{PrN}\right)_{2} \mathrm{Cu}\right]_{2}+\mathrm{LiCl},
$$

where $\mathrm{R}=\mathrm{Me}_{2} \mathrm{~N}$ (1) and ${ }^{\mathrm{i}} \operatorname{PrN}(\mathrm{H})$ (2). These compounds formed in excellent yields (96\% 1, $89 \%$ 2), and the simplicity of their ${ }^{1} \mathrm{H}$ NMR spectra suggested a symmetrical structure in both cases. Single X-ray quality crystals for both $\mathbf{1}$ and $\mathbf{2}$ were grown from $\mathrm{Et}_{2} \mathrm{O}$, and their structures were determined (Figure 2.3, Tables 2.1 and Tables 2.2).
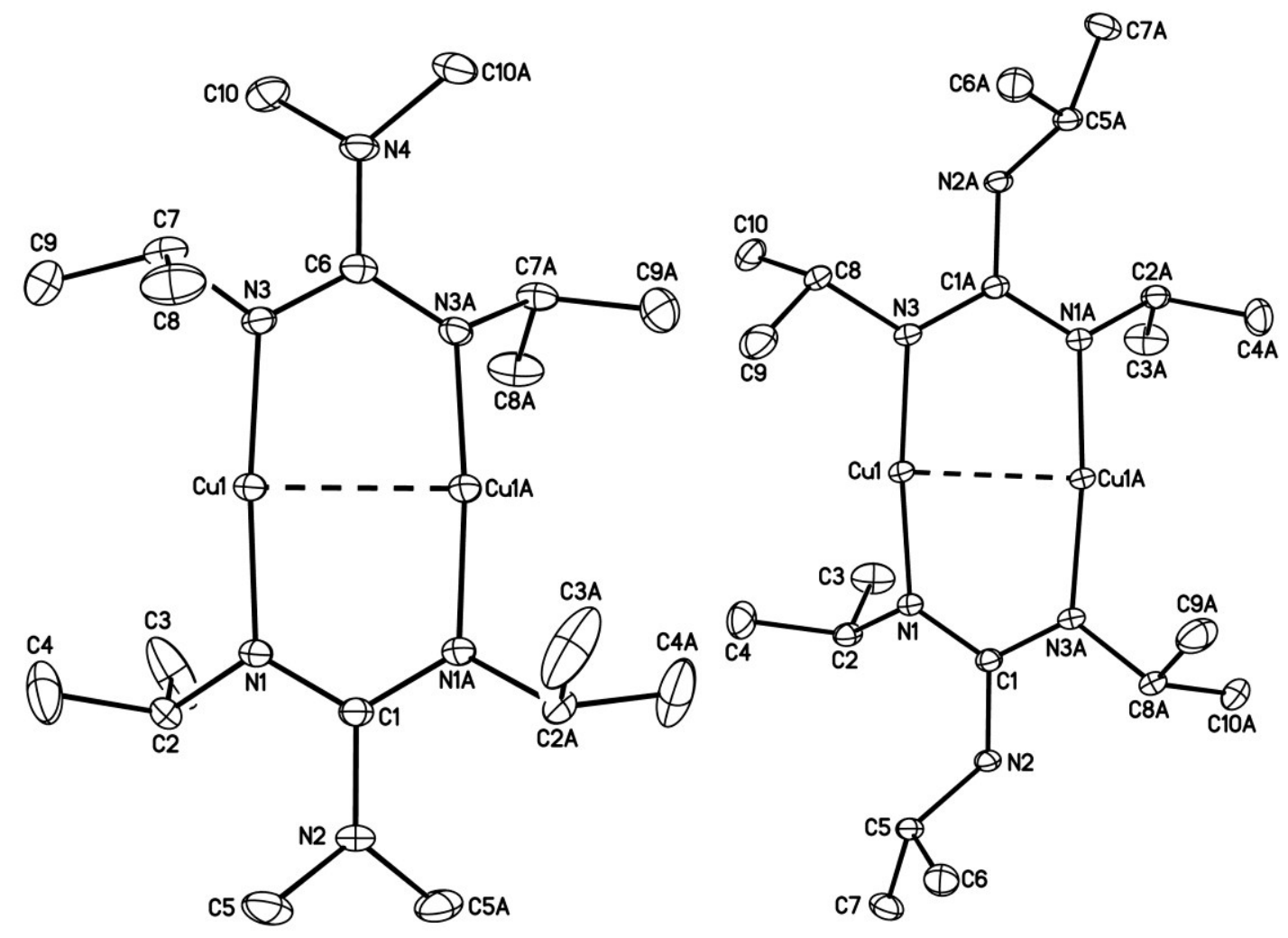

Figure 2.3 Single crystal X-ray structures for compounds $\mathbf{1}$ and 2. Hydrogen atoms were omitted for clarity, and the thermal ellipses are shown at $30 \%$.

Both compounds have a $\mathrm{C}_{2}$ axis perpendicular to the $\mathrm{Cu}-\mathrm{Cu}$ axis. The $\mathrm{C}_{2}$ axis in $\mathbf{1}$ is parallel to the mean plane of the $\mathrm{N}$ atoms; in $\mathbf{2}$ it is perpendicular to the nitrogen atom plane. The $\mathrm{Cu}-\mathrm{N}$ bond lengths for both compounds are within the range $1.87-1.88 \AA$, 
which agrees well with copper amidinates that have the same dimeric structure. ${ }^{8,9}$ The $\mathrm{Cu}-\mathrm{Cu}$ distances in $\mathbf{1}$ and $\mathbf{2}$ were 2.42 and $2.43 \AA$, respectively. This falls within the range of general $\mathrm{Cu}-\mathrm{Cu}$ bonds with bridging ligands, and is much shorter than the $\mathrm{Cu}-\mathrm{Cu}$ distance in metallic copper. ${ }^{13}$ These are intermediate to the reported copper distances in the amidinate dimers $(2.40 \AA$ ( ref 8) and $2.46 \AA$ (ref 9)). The $\mathrm{C}-\mathrm{N}-\mathrm{C}$ angles are also similar $\left(120.0^{\circ}\right.$ for $\mathbf{1}$ and $120.5^{\circ}$ for $\mathbf{2}$, compared to $119.6^{\circ}$ (ref 8 ) for $\mathbf{1}$ to $120.1^{\circ}$ (ref 9 ) for 2 .

The guanidinate compounds' structures differ in that the ligand-copper plane is noticeably twisted, and this can be highlighted by considering the N1-N1A-N3-N3A torsion angle $\left(32.0^{\circ}\right.$ for $\mathbf{1}$ and $37.5^{\circ}$ for 2; Figure 2.4$)$, while previously reported amidinates are much more planar $\left(2.0^{\circ}(\operatorname{ref} 8)\right.$ and $\left.0^{\circ}(\operatorname{ref} 9)\right)$.

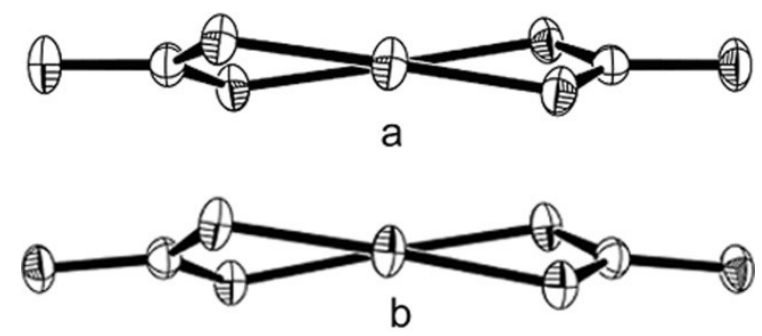

Figure 2.4 The dimer cores of (a) 1 and (b) 2, showing the twists induced by pyramidalization of the chelating nitrogens. Except for the ligands' $\mathrm{sp}^{2}$ carbon, all carbon and hydrogen atoms are omitted for clarity, and the thermal ellipses are shown at $30 \%$.

This core twist can be attributed to the participation of the exocyclic amido moiety of the guanidinates participating in the $\pi$ system of the chelate ring, effectively allowing the amides to be slightly pyramidalized (Figure 2.1, structure C). This is common for guanidinate ligands. ${ }^{11}$ 
Table 2.1 Selected Crystal Data and Structure Refinement Parameters

\begin{tabular}{lll}
\hline & \multicolumn{1}{c}{$\mathrm{C}_{18} \mathrm{H}_{40} \mathrm{Cu}_{2} \mathrm{~N}_{6}(\mathbf{1})$} & \multicolumn{1}{c}{$\mathrm{C}_{20} \mathrm{H}_{44} \mathrm{Cu}_{2} \mathrm{~N}_{6}(\mathbf{2})$} \\
\hline $\mathrm{fw}$ & 467.64 & 495.69 \\
$T(\mathrm{~K})$ & 120 & 120 \\
$\lambda(\AA)$ & 0.71073 & 0.71073 \\
cryst syst & orthorhombic & monoclinic \\
space group & $\mathrm{C} 222_{1}$ & $\mathrm{C} 2 / \mathrm{c}$ \\
$\mathrm{a}(\AA)$ & 11.329 & 23.939 \\
$\mathrm{~b}(\AA)$ & 21.308 & 5.943 \\
$\mathrm{c}(\AA)$ & 9.923 & 21.238 \\
$\alpha(\mathrm{deg})$ & 90.00 & 90.00 \\
$\beta(\mathrm{deg})$ & 90.00 & 122.45 \\
$\gamma(\operatorname{deg})$ & 90.00 & 90.00 \\
$V\left(\AA^{3}\right)$ & 2395 & 2550 \\
$\mathrm{Z}$ & 4 & 4 \\
$\rho_{\text {calcd }}\left(\mathrm{mg} / \mathrm{m}^{3}\right)$ & 1.297 & 1.291 \\
abs coeff $\left(\mathrm{mm}^{-1}\right)$ & 1.788 & 1.684 \\
refinement method & full-matrix least-squares on $F^{2}$ & full-matrix least-squares on $F^{2}$ \\
$R$ indices $[\mathrm{I}>2 \sigma(I)]^{\mathrm{a}}$ & $\mathrm{R} 1=0.0360, \mathrm{wR} 2=0.0860$ & $\mathrm{R} 1=0.0440, \mathrm{wR} 2=0.1068$ \\
\hline${ }^{\mathrm{a}} \mathrm{R} 1=\Sigma|| \mathrm{F}_{\mathrm{o}}|-| \mathrm{F}_{\mathrm{c}}|| \Sigma\left|\mathrm{F}_{\mathrm{o}}\right|$ and $\mathrm{wR} 2=\left(\sum w\left(\left|\mathrm{~F}_{\mathrm{o}}\right|-\mid \mathrm{F}_{\mathrm{c}}\right)^{2} / \Sigma w\left|\mathrm{~F}_{\mathrm{o}}\right|^{2}\right)^{1 / 2}$.
\end{tabular}


Table 2.2 Selected bond lengths and angles for compounds $\mathbf{1}$ and $\mathbf{2}$.

\begin{tabular}{|c|c|c|c|}
\hline & 1 & & 2 \\
\hline \multicolumn{4}{|c|}{ selected bond lengths $(\AA)$} \\
\hline Cu1-N1 & $1.875(2)$ & $\mathrm{Cu} 1-\mathrm{N} 1$ & $1.875(2)$ \\
\hline $\mathrm{Cu} 1-\mathrm{N} 3$ & $1.876(2)$ & $\mathrm{Cu} 1-\mathrm{N} 3$ & $1.871(2)$ \\
\hline $\mathrm{Cu} 1-\mathrm{Cu} 1 \mathrm{~A}$ & $2.4233(10)$ & $\mathrm{Cu} 1-\mathrm{Cu} 1 \mathrm{~A}$ & $2.4289(11)$ \\
\hline $\mathrm{N} 1-\mathrm{C} 1$ & $1.341(3)$ & $\mathrm{N} 1-\mathrm{C} 1$ & $1.338(3)$ \\
\hline N3-C6 & $1.334(3)$ & $\mathrm{N} 3-\mathrm{C} 1$ & $1.345(3)$ \\
\hline $\mathrm{N} 2-\mathrm{C} 1$ & $1.396(5)$ & $\mathrm{N} 2-\mathrm{C} 1$ & $1.399(3)$ \\
\hline $\mathrm{N} 4-\mathrm{C} 6$ & $1.411(5)$ & & \\
\hline \multicolumn{4}{|c|}{ selected bond angles (deg) } \\
\hline $\mathrm{N} 1-\mathrm{Cu} 1-\mathrm{N} 3$ & $174.75(10)$ & $\mathrm{N} 1-\mathrm{Cu} 1-\mathrm{N} 3$ & $173.71(8)$ \\
\hline C7-N3-Cu1 & $117.14(18)$ & $\mathrm{C} 8-\mathrm{N} 3-\mathrm{Cu} 1$ & $121.38(15)$ \\
\hline $\mathrm{C} 2-\mathrm{N} 1-\mathrm{Cu} 1$ & $118.66(18)$ & C2-N1--Cu1 & $117.93(13)$ \\
\hline N3-C6-N3A & $120.4(3)$ & $\mathrm{N} 1-\mathrm{C} 1-\mathrm{N} 3 \mathrm{~A}$ & $120.51(19)$ \\
\hline N1-C1-N1A & $120.4(3)$ & & \\
\hline \multicolumn{4}{|c|}{ sum of angles (deg) } \\
\hline N1 & 358.2 & N1 & 357.8 \\
\hline N3 & 357.3 & N3 & 360 \\
\hline N2 & 360 & $\mathrm{C} 1$ & 359.9 \\
\hline N4 & 360 & & \\
\hline $\mathrm{C} 1$ & 360 & & \\
\hline $\mathrm{C} 6$ & 360 & & \\
\hline \multicolumn{4}{|c|}{ torsion angles (deg) } \\
\hline N1-N1A-N3- & 3 A 32 & $\mathrm{~N} 1-\mathrm{N} 3 \mathrm{~A}-\mathrm{N} 3-\mathrm{N}$ & A 37.5 \\
\hline
\end{tabular}

The guanidinate compounds were studied to determine their thermal chemistry, and compared to the previously reported $\left[\mathrm{MeC}\left({ }^{i} \mathrm{PrN}\right)_{2} \mathrm{Cu}\right]_{2}(\mathbf{3}) \cdot{ }^{8}$ The thermolysis study was performed in three stages.

Initially, thermogravimetric analyses (TGA) were collected for each compound to determine their volatility (Figure 2.5 and Table 2.3 ). All three compounds showed very similar volatilization, even though their melting points differed significantly. This is also reflected in the onset of volatilization of the compounds, which did not follow the 
melting point trend. As well, each compound showed very low residual mass $(<5 \%$ in all cases). These experiments suggest that all three precursors would be equally good copper precursors, and might lead one to assume that their deposition and thermal decomposition chemistries were similar. However, further thermolysis studies revealed that the thermal chemistry of the copper guanidinates differed significantly from that of the copper amidinate.

Table 2.3 Thermal Decomposition Data for $\mathbf{1}$ - 3 .

\begin{tabular}{lccc}
\hline & $\mathbf{1}$ & $\mathbf{2}$ & $\mathbf{3}$ \\
\hline melting point $\left({ }^{\circ} \mathrm{C}\right)$ & 110 & 79 & 147 \\
onset of volatilization ${ }^{\mathrm{a}}\left({ }^{\circ} \mathrm{C}\right)$ & 105 & 87 & 102 \\
TGA residual mass $(\%)$ & 1.8 & 4.6 & 2.2 \\
initial copper deposition, NMR tube $\left({ }^{\circ} \mathrm{C}\right)$ & 125 & 125 & 135 \\
onset of deinsertion, NMR tube $\left({ }^{\circ} \mathrm{C}\right)$ & 135 & 135 & -- \\
onset of deinsertion, tube furnace $\left({ }^{\circ} \mathrm{C}\right)$ & 225 & 250 & -- \\
\hline
\end{tabular}

${ }^{\mathrm{a}}$ Onset of volatilization is considered to be the point in the TGA where $1 \%$ of the mass is lost.

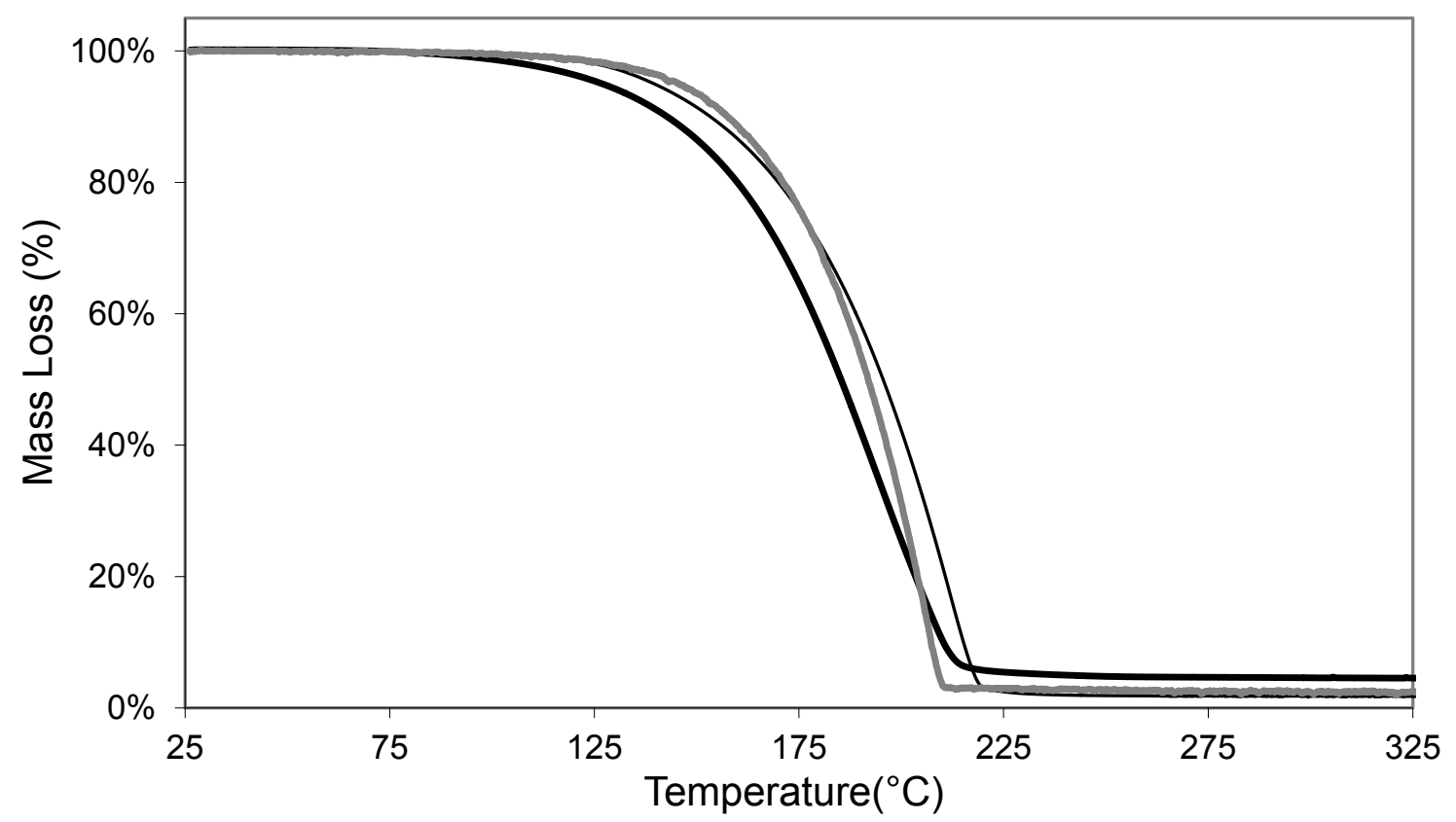

Figure 2.5 Thermogravimetric analyses of 1 (solid line), 2 (bold line), and $\mathbf{3}$ (grey line). 
Each compound was subjected to a sealed tube thermolysis, wherein a sealed, heavy walled NMR tube was heated in a tube furnace at intervals from room temperature to a maximum of $235{ }^{\circ} \mathrm{C}$. Periodically, ${ }^{1} \mathrm{H}$ NMR spectra were collected to determine what, if any, thermolysis products were identifiable. For compounds $\mathbf{1}$ and $\mathbf{2}$, characteristic ${ }^{1} \mathrm{H}$ NMR peaks for diisopropylcarbodiimide (CDI) plus dimethylamine for $\mathbf{1}$ and isopropylamine for $\mathbf{2}$ were seen at $135^{\circ} \mathrm{C}$, and subsequently increased in intensity at higher temperatures. Deinsertion of CDI from guanidinate ligands is a prevalent thermolysis route. ${ }^{11,12}$ Additional support for this thermolysis route was seen by FTIR spectroscopy. For both $\mathbf{1}$ and 2, FT-IR analysis of the solution resulting from the NMR tube thermolysis showed an absorbance at $2120 \mathrm{~cm}^{-1}$, which is indicative of the asymmetric stretch of CDI. Very tellingly, copper metal plated out of solution and deposited on the walls of the NMR tube in both cases, showing that (in solution, at least) compounds $\mathbf{1}$ and $\mathbf{2}$ are viable precursor for copper metal without requiring a reducing agent.

The most likely decomposition mechanism for these guanidinates is the deinsertion of carbodiimide. This deinsertion is followed by decomposition of the resulting copper (I) amide through deprotonation of the amide by another amide to produce an imine (Figure 2.6). This reactivity has been seen previously by Saegusa. ${ }^{14}$ Peaks in the FTIR of the thermolysis solution also support this reaction scheme. The FTIR showed a peak at $1639 \mathrm{~cm}^{-1}$ for both $\mathbf{1}$ and $\mathbf{2}$ that is attributed to the imides resulting from copper amide deprotonation. The corresponding amines were not seen in the IR, but we speculate that they evaporated during the IR plate preparation. 


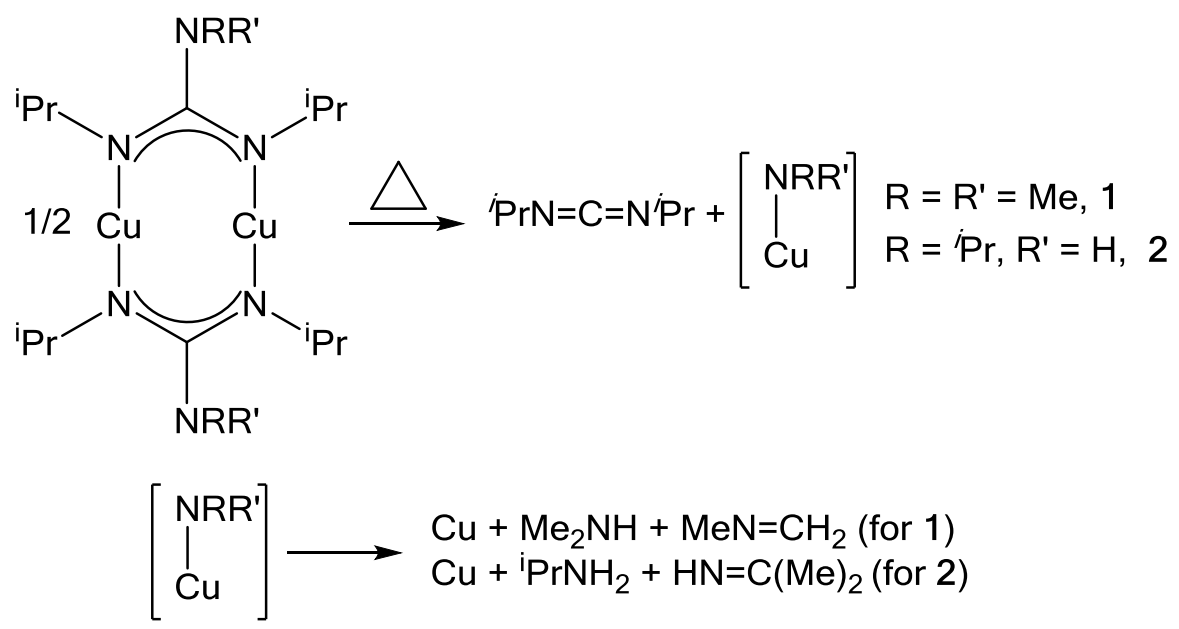

Figure 2.6 Thermal Decomposition of Copper Guanidinates.

We were interested to see if CDI deinsertion was occurring in the vapour phase for the guanidinate compounds, and whether the amidinate species would show any CDI deinsertion at elevated temperatures. Using a furnace coupled to a quadrapole mass spectrometer, we able to collect temperature-resolved mass spectra. This experiment is much more indicative of the environment that the precursors would be subjected to in a CVD or ALD experiment: the precursors are thermolysed at low concentration in the gas phase, and exposed to the thermolysis zone for a relatively short period of time. Thus, we anticipated that the thermolysis chemistry would correlate to the TGA, as well as the solution thermolysis, but the reaction temperatures would not correspond exactly to the vapour phase experiments.

For 1, a mass signal for the free guanidine (171 amu) was seen to decrease significantly at $225^{\circ} \mathrm{C}$, with a corresponding increase in the mass signal for carbodiimide (126 amu) (Figure 2.7). This trend is also apparent in ionization fragments of the carbodiimide, notably mass $111 \mathrm{amu}(\mathrm{CDI}-\mathrm{Me})$. This suggested that at temperatures below $225^{\circ} \mathrm{C}$, the whole precursor is surviving to reach the mass spectrometer, and 
fragmentation in the mass spectrometer produces the guanidine ion. At $225^{\circ} \mathrm{C}$, CDI begins to thermally deinsert while the precursor is in the tube furnace, and thus it and its fragments become prominent in the mass spectrum above this temperature. It should be noted that the CDI is seen to diminish above about $300^{\circ} \mathrm{C}$, where diisopropyl carbodiimide itself undergoes thermal decomposition.

Although this temperature was greater than the $125-135^{\circ} \mathrm{C}$ measured in the sealed tube thermolysis, this is likely a kinetic effect due to the low residence time of the precursor in the thermolysis zone during the vapour phase experiment. This is a good indication that this species could be used for ALD deposition below $225{ }^{\circ} \mathrm{C}$, as thermal decomposition precludes ALD deposition. Above $225^{\circ} \mathrm{C}$, this species might be used as a CVD precursor for copper metal without requiring a reducing agent.

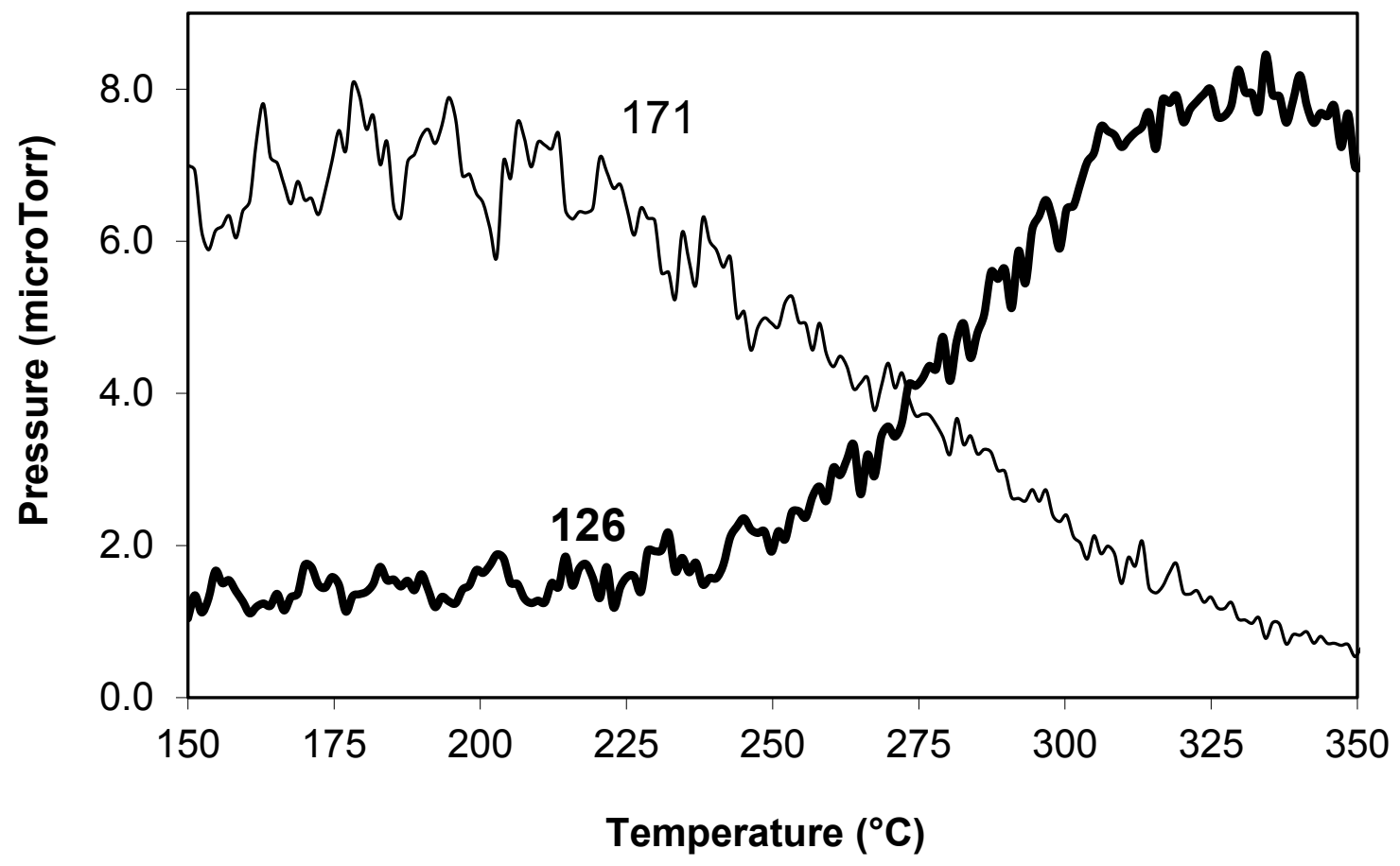

Figure 2.7 Temperature-resolved mass spectrum of 1 showing the increase in carbodiimide and a corresponding decrease in the dimethylamido diisopropyl guanidine. 
For 2, the mass signal for the protonated guanidine cation (186 amu) was seen to decrease significantly at $200{ }^{\circ} \mathrm{C}$ (Figure 2.8). The fragments of this species also echoed this trend. At the same time, the mass signal for CDI (126 amu) started to ramp in very slowly at approximately $225^{\circ} \mathrm{C}$. The fragments of this species follow this trend, and were again most obvious for the mass $111 \mathrm{amu}$. This again suggested that the whole precursor is surviving to reach the mass spectrometer until temperatures higher than $225^{\circ} \mathrm{C}$, where deinsertion starts to play a significant role, similar to 1 . Interestingly, the amidinate $\mathbf{3}$ gave very different thermal behaviour, even though its thermal chemistry occurred at similar temperatures (Figure 2.5 and Table 2.3).

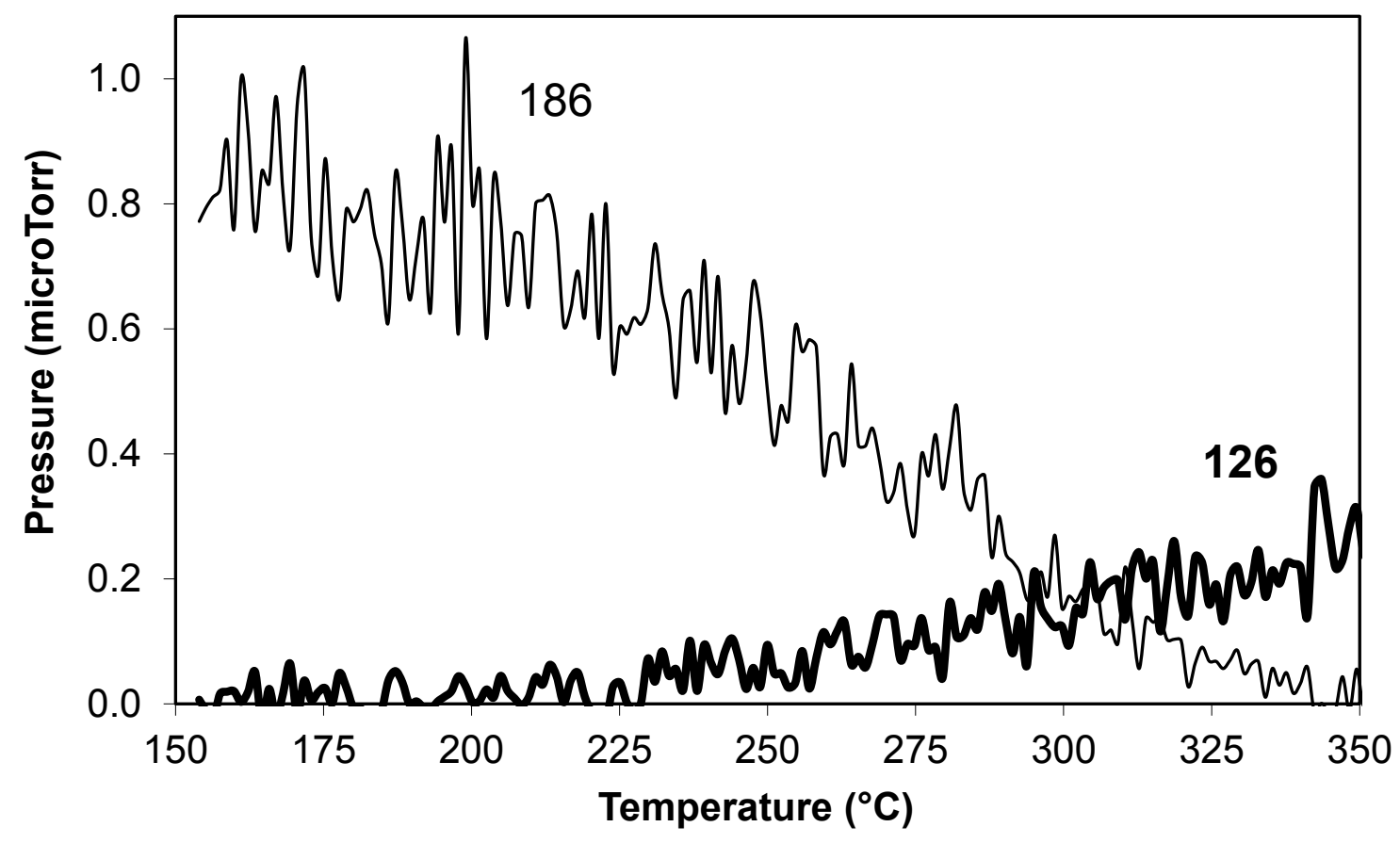

Figure 2.8 Temperature-resolved mass spectrum of $\mathbf{2}$ showing the increase in carbodiimide and a corresponding decrease in the protonated triisopropyl guanidine cation.

The NMR tube thermolysis experiment showed no characteristic isopropyl signals for CDI in the ${ }^{1} \mathrm{H}$ NMR, even up to $235^{\circ} \mathrm{C}$, the practical limit of this thermolysis 
experiment. Additionally, there was no evidence of carbodiimide in solution FT-IR. However, copper metal was seen to deposit on the walls of the NMR tube at $135^{\circ} \mathrm{C}$.

The ${ }^{1} \mathrm{H}$ NMR showed no new signals until $235^{\circ} \mathrm{C}$ where peaks corresponding to the amidine ligand appeared. Further thermolysis experiments in a sealed pressure vessel at $235^{\circ} \mathrm{C}$ allowed the isolation of a small amount of air-sensitive, colourless oil as well as deposited metallic copper. This oil tested positive for copper using a qualitative flame test, and a GC-MS of the oil showed a single peak at $143 \mathrm{amu}$, which corresponds with the mass of the protonated amidine ligand.

Although unambiguous characterization of this oil was not achieved due to its extreme reactivity and very low isolated mass, we suspect this might be a paramagnetic homoleptic copper (II) diisopropyl acetamidinate, liberated through disproportionation of the copper (I) precursor.

$$
\left[\mathrm{MeC}\left({ }^{i} \mathrm{PrN}\right)_{2} \mathrm{Cu}\right]_{2} \rightarrow \mathrm{Cu}(0)+\left(\mathrm{MeC}\left({ }^{i} \mathrm{PrN}\right)_{2}\right)_{2} \mathrm{Cu}
$$

We suspect further thermolysis of the copper (II) acetamidinate leads to protonated amidine ligand by an unknown decomposition pathway. Similar reactivity has recently been reported for copper (I) betadiketiminates ${ }^{15}$ as well as previously in copper (I) betadiketonate chemistry. ${ }^{16}$ This reactivity needs to be further disambiguated, and is a topic of research in our laboratory.

Compound $\mathbf{3}$ also showed no evidence of carbodiimide deinsertion in the temperature-resolved MS, to the maximum temperature of this experiment. The characteristic parent and fragment mass signals at 126 and 111 remaining undetectably close to zero throughout the temperature range. However, a mass signal for the parent guanidine cation peak (143 amu) started to decline at about $190{ }^{\circ} \mathrm{C}$ with a corresponding 
increase in the mass signal for the fragment " $\mathrm{Pr}-\mathrm{N}=\mathrm{C}$ " (69 amu) (Figure 2.9). This suggests that the main thermal decomposition pathway for the amidinate is not by CDI deinsertion, but by destruction of the amidinate itself. Unfortunately, the vapour-phase thermolysis experiment could not identify the potential copper (II) amidinate from disproportionation, as the mass of this species is above the limit of the mass spectrometer.

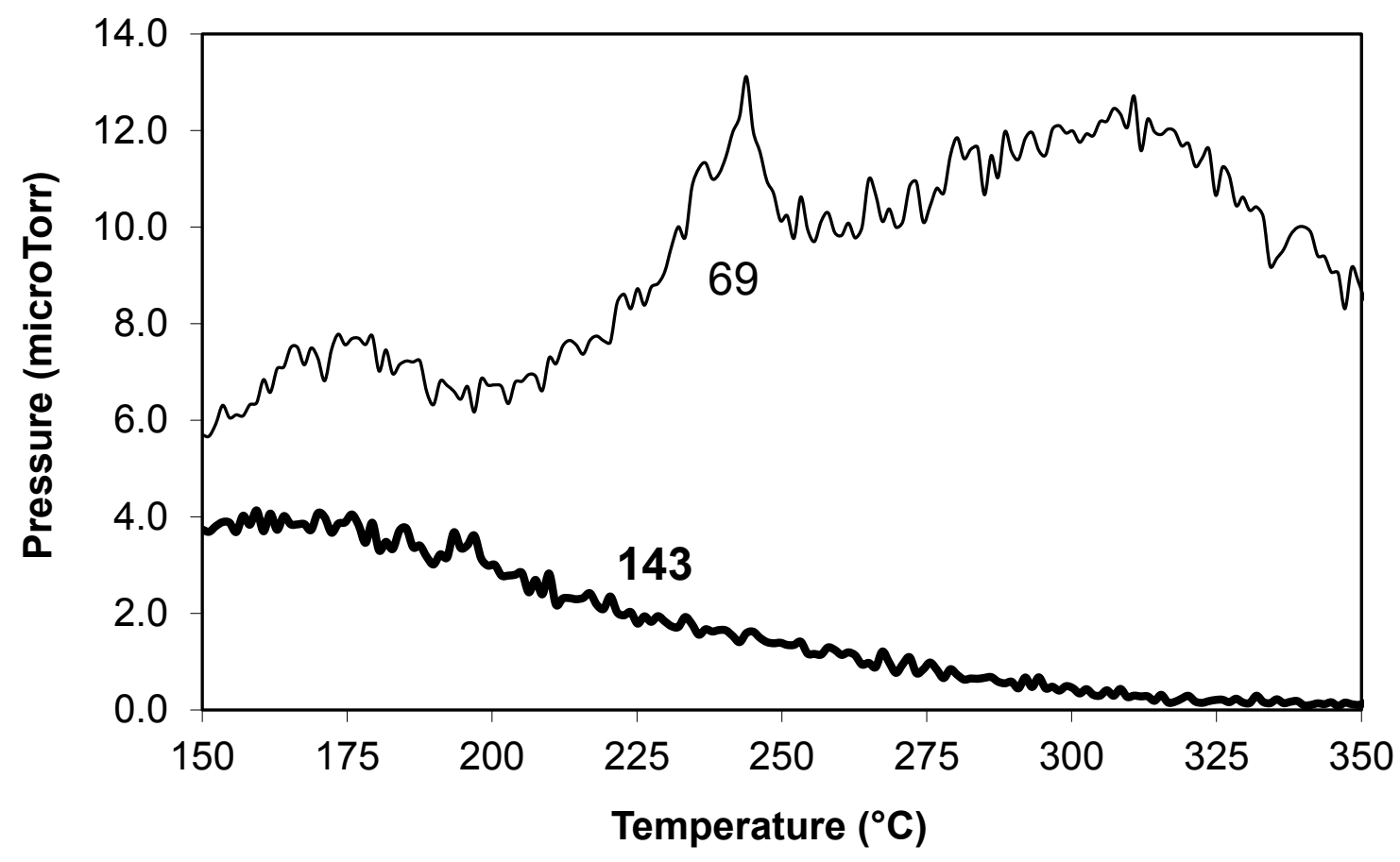

Figure 2.9 The temperature-resolved mass spectrum of $\mathbf{3}$ showing the increase in the ligand fragment " $\mathrm{C}=\mathrm{N}^{\mathrm{i}} \mathrm{Pr}$ " and a corresponding decrease in the protonated acetamidine cation.

Simple CVD experiments were undertaken to demonstrate the utility of $\mathbf{1}$ and $\mathbf{2}$ as precursors. The depositions were performed in a tube furnace at $225^{\circ} \mathrm{C}$ under roughing pump vacuum ( $\sim 10^{-3}$ Torr). Nitrogen carrier gas was used ( $\left.20 \mathrm{sccm}\right)$, and this was pulsed on and off at 3 second intervals. The pulsing was necessary to promote volatilization of the copper precursor due to the design of the reactor. Silicon (111) with a native oxide coating was used as a substrate. 
Both guanidinate precursors produced metallic, copper-coloured films. Scanning electron micrographs showed the films to be microcrystalline to nanocrystalline, which is commonly the case with vapour-deposited copper (Figure 2.10). ${ }^{17}$ X-ray diffraction identified the films as polycrystalline copper in both cases (Figure 2.11), and energy dispersive X-ray corroborated this fact. The growth is quite different in each case, with 2 showing much larger crystallite formation, a wide variety of shapes. The film grown by $\mathbf{1}$ consisted mainly of $>100 \mathrm{~nm}$ crystallites with a faceted "boulder" appearance, while the film grown from 2 showed "boulders" up to $500 \mathrm{~nm}$, as well as rods, some as long as 2 $\mu \mathrm{m}$.
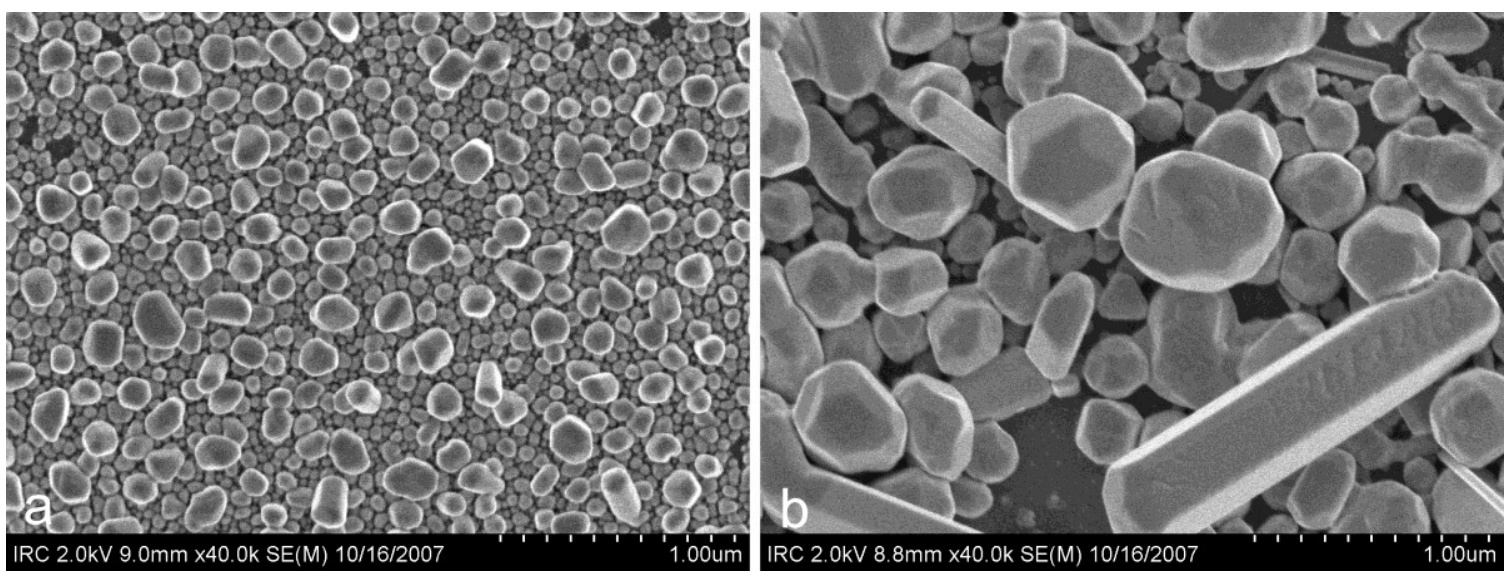

Figure 2.10 Scanning electron micrograph of copper films deposited by (a) 1 and (b) 2 . 


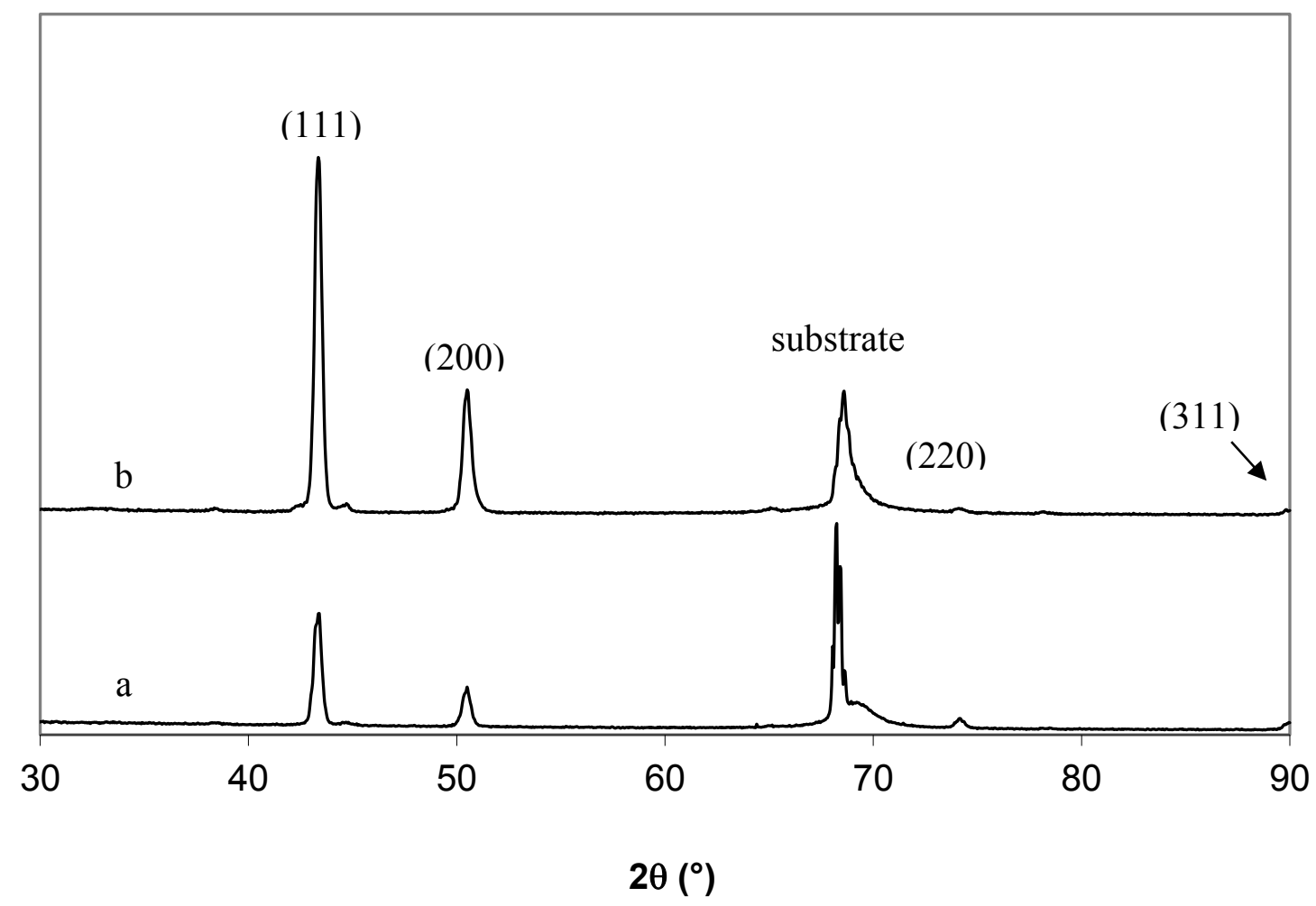

Figure 2.11 X-ray diffraction spectra of copper deposited in a CVD experiment using (a) $\mathbf{1}$ and (b) $\mathbf{2}$ as a precursor.

The mass spectrometer data for each compound supported the decomposition pathway suggested in Figure 2.6. The mass spectra showed parent peaks for the guanidinate ligands, as well as for CDI and its fragmentation products. The partial pressure for CDI was higher then that of the parent guanidinate ligands, which is a sign of deinsertion occurring before fragmentation in the mass spectrometer. Ideally, deposition of copper by a CDI deinsertion decomposition pathway would show only CDI as a significant peak in the in situ QMS data; however, a partial pressure of the guanidinate ligand was detectable during deposition but this was not surprising considering that the vaporous precursor is unlikely to be totally consumed by deposition. In the case of $\mathbf{1}$, a fragment with a mass of 44 amu was abundant in the mass spectrum, registering as high 
as $2 \times 10^{-4}$ Torr, which was a very high partial pressure compared to other mass channels. This could be attributed to the protonated form of the fragment $\mathrm{MeN}=\mathrm{CH}_{2}$, as speculated in Figure 2.6. Similarly for $\mathbf{2}$, a fragment with a mass of $58 \mathrm{amu}$ showed a partial pressure of $2 \times 10^{-5}$ Torr and was attributed to the protonated form of the fragment $\mathrm{HN}=\mathrm{CMe}_{2}$ (Figure 2.6). It should be noted that these masses are common fragments of their respective ligands, and thus we are presently studying this thermal decomposition pathway more closely to verify our suggested mechanism.

\subsection{Conclusion}

The copper (I) guanidinate compounds show a similar response to temperature as the copper (I) amidinate: they deposit copper metal at temperatures just above $100{ }^{\circ} \mathrm{C}$ in a sealed tube experiment. However, the guanidinates do so through a carbodiimide deinsertion reaction, while the amidinate undergoes a more complicated thermolysis, potentially involving the disproportionation of copper (I) to produce metallic copper and copper (II). Temperature-resolved mass spectrometry showed that the thermolysis of both the amidinate and guanidinates decomposed at higher temperatures in the dilute gas phase, albeit by mechanisms that were similar to their respective sealed-tube experiments. We attribute the higher decomposition temperature in the gas-phase experiment to the short exposure time of the precursor to an elevated temperature. This better emulates the conditions these species would encounter during a typical ALD experiment, and thus allows the determination of the temperature limit for these species as ALD precursors. Finally, both compounds $\mathbf{1}$ and $\mathbf{2}$ showed deposition of copper metal at $225^{\circ} \mathrm{C}$ without a secondary gas, demonstrating their utility as CVD precursors for copper metal films. 


\subsection{Experimental}

General Considerations: All manipulations were carried out in an MBraun Unilab inert atmosphere drybox. $\operatorname{Li}\left({ }^{i} \mathrm{PrN}\right)_{2} \mathrm{CN}(\mathrm{H}){ }^{i} \operatorname{Pr}$ was synthesized following literature preparation for general guanidinate synthesis. ${ }^{18}$ Lithium dimethyl amide, diisopropyl carbodiimide, copper (I) chloride were purchased from Aldrich Chemical Company and used as received. Hexanes and diethyl ether were purified with respect to oxygen and water using an MBraun solvent purification system, and stored on activated 4A sieves until used. Guelph Chemical Laboratories in Guelph, Ontario, Canada, performed combustion analyses.

$\left[\mathrm{Me}_{2} \mathrm{NC}(\mathrm{PrN})_{2} \mathrm{Cu}\right]_{2}$ (1): In a $50 \mathrm{~mL}$ flask, $\mathrm{LiNMe}_{2}(0.274 \mathrm{~g}, 5.10 \mathrm{mmol})$ was suspended in $30 \mathrm{~mL}$ of hexanes. Diisopropylcarbodiimide $(0.668 \mathrm{~g}, 5.30 \mathrm{mmol})$ was diluted in $8 \mathrm{~mL}$ of hexanes and added dropwise to the suspension of $\mathrm{LiNMe}_{2}$. The cloudy, pale yellow suspension cleared to a homogeneous pale yellow solution over $2 \mathrm{~h}$ of stirring. $\mathrm{CuCl}(0.521 \mathrm{~g}, 5.10 \mathrm{mmol})$ was added and the mixture was stirred for $18 \mathrm{~h}$, after which a cloudy light green solution was filtered to afford a light green solid and a slightly pale yellow solution. Volatiles were removed under reduced pressure to afford a crystalline off-white solid (1.14 g, 95.9 \% crude yield). The solid was dissolved in a minimal volume of diethyl ether and was kept at $-30{ }^{\circ} \mathrm{C}$ overnight. Compound 1 was collected as clear, colourless crystals $(0.86 \mathrm{~g}, 72 \%)$. mp $109{ }^{\circ} \mathrm{C}$. Anal. Calcd for $\mathrm{C}_{18} \mathrm{H}_{40} \mathrm{Cu}_{2} \mathrm{~N}_{6}: \mathrm{C}, 46.23 ; \mathrm{H}, 8.62 ; \mathrm{N}, 17.97$. Found: C, 46.51; H, 9.01; N, 18.22 Mass spectra m/e (relative abundance): $466(0.1) \mathrm{M}^{+} .{ }^{1} \mathrm{H}$ NMR $\left(300 \mathrm{MHz}, \mathrm{C}_{6} \mathrm{D}_{6}\right): \delta 3.41$ [sept, $\left.4 \mathrm{H}, \mathrm{NCH} H^{\mathrm{i}} \mathrm{Pr}\right], \delta 2.54\left[\mathrm{~s}, 12 \mathrm{H}, \mathrm{N}\left(\mathrm{CH}_{3}\right)_{2}\right], \delta 1.29\left[\mathrm{~d}, 24 \mathrm{H} \mathrm{NCH}\left(\mathrm{CH}_{3}\right)_{2}\right] .{ }^{13} \mathrm{C} \mathrm{NMR}(75 \mathrm{MHz}$, $\left.\mathrm{C}_{6} \mathrm{D}_{6}\right): \delta 48.22\left[\mathrm{CHCH}_{3}\right], \delta 27.59\left[\mathrm{CHCH}_{3}\right], \delta 40.92\left[\mathrm{~N}\left(\mathrm{CH}_{3}\right)_{2}, \delta 171.58\left[\left({ }^{\mathrm{i}} \mathrm{PrN}\right)_{2} C \mathrm{NMe}_{2}\right]\right.$. 
$\left.{ }_{[}^{i} \operatorname{PrN}(\mathrm{H}) \mathrm{C}\left({ }^{i} \operatorname{PrN}\right)_{2} \mathrm{Cu}\right]_{2}$ (2): In a $250 \mathrm{~mL}$ flask, $\mathrm{Li}\left({ }^{i} \operatorname{PrN}\right)_{2} \mathrm{CN}(\mathrm{H}){ }^{i} \operatorname{Pr}(7.727 \mathrm{~g}, 40.4$ mmol) was added to a suspension of $\mathrm{CuCl}(4.0 \mathrm{~g}, 40.4 \mathrm{mmol})$ in $100 \mathrm{~mL}$ hexanes. The solution was stirred for $18 \mathrm{~h}$. It was filtered to afford a pale yellow, clear solution. Volatiles were removed under reduced pressure to yield compound $\mathbf{2}$ as off-white crystalline mass $(8.9 \mathrm{~g}, 89 \%)$. The crude product was recrystallized from $10 \mathrm{ml}$ diethyl ether to afford clear, colourless crystals, $(6.69 \mathrm{~g}, 67 \%) \mathrm{mp} 79{ }^{\circ} \mathrm{C}$ Anal. Calcd for $\mathrm{C}_{18} \mathrm{H}_{40} \mathrm{Cu}_{2} \mathrm{~N}_{6}: \mathrm{C}, 48.46 ; \mathrm{H}, 8.95 ; \mathrm{N}, 16.95$. Found: $\mathrm{C}, 48.36 ; \mathrm{H}, 9.17 ; \mathrm{N}, 16.58$ Mass spectra m/e (relative abundance): $494(0.1) \mathrm{M}^{+} .{ }^{1} \mathrm{H}$ NMR $\left(300 \mathrm{MHz}, \mathrm{C}_{6} \mathrm{D}_{6}\right): \delta 3.64$ [sept, 4H, $\mathrm{NCHMe}$ ], $\delta 3.46$ [sept, 2H, $\mathrm{HNCHMe}_{2}$ ], $\delta 3.06$ [d, 2H $H \mathrm{NCHMe}_{2}$ ], $\delta 1.30$ [d, 24H $\left.\mathrm{NCH}\left(\mathrm{CH}_{3}\right)_{2}\right], \delta 0.97\left[\mathrm{~d}, 12 \mathrm{H} \mathrm{HNCH}\left(\mathrm{CH}_{3}\right)_{2}\right] .{ }^{13} \mathrm{C} \mathrm{NMR}\left(75 \mathrm{MHz}, \mathrm{C}_{6} \mathrm{D}_{6}\right): \delta 27.67$ $\left[\mathrm{NCHCH}_{3}\right], \delta 47.49\left[\mathrm{NCHCH}_{3}\right], \delta 23.55\left[\mathrm{HNCH}\left(\mathrm{CH}_{3}\right)_{2}, \delta 48.44\left[\mathrm{HNCH}\left(\mathrm{CH}_{3}\right)_{2}, \delta 171.58\right.\right.$ $\left[\left({ }^{\mathrm{i}} \mathrm{PrN}\right)_{2} \mathrm{CNMe}{ }_{2}\right]$.

Crystallography: Crystals were selected and mounted on plastic loops with viscous oil and cooled to the data collection temperature. Diffraction data were collected on a Bruker-AXS APEX CCD diffractometer. All data-sets were treated with SADABS absorption corrections. Unit-cell parameters were determined by sampling three different sections of the Ewald sphere. The systematic absences in the diffraction data were consistent for $C c$ and $C 2 / c$ for $\mathbf{2}$ and, uniquely, for $C 222_{1}$ for $\mathbf{1}$. Structural solution in the centrosymmetric space group option for $\mathbf{2}$ yielded chemically reasonable and computationally stable results of refinement. The anomalous dispersion factor refined to nil in $\mathbf{1}$ indicating that the true hand of the data was determined. The compound molecules were located on twofold symmetry. All non-hydrogen atoms were refined with 
anisotropic displacement parameters. All hydrogen atoms were treated as idealized contributions. Structure factors are contained in SHELXTL 6.12 program library. ${ }^{19}$

Sealed NMR tube thermolysis: NMR samples of 1, 2, and 3 were prepared in thick-walled NMR tubes using $\mathrm{C}_{6} \mathrm{D}_{6}$ as solvent. The tubes were sealed while frozen in liquid nitrogen and under reduced pressure. The three samples were left overnight in a furnace at the following temperatures $100,125,135,150,175,190,205,225$ and $235^{\circ} \mathrm{C}$. After each thermolysis, a ${ }^{1} \mathrm{H}$ NMR was collected

Gas Phase Thermolysis (RGA):A bubbler was charged with the compound of interest and heated to $5^{\circ} \mathrm{C}$ above the compound's melting point to increase vapour pressure. This was coupled through a high-vacuum fitting to our homemade ALD reactor, which was run with all of its valves open and with no carrier gas. The vapour was carried through a tube furnace that was ramped from $150{ }^{\circ} \mathrm{C}$ to $450{ }^{\circ} \mathrm{C}$ at $3{ }^{\circ} / \mathrm{min}$. The resulting gas phase species were sampled by a Microvision Plus 1000 e "SmartHead" 300 amu quadropole mass spectrometer, thus allowing a temperature-resolved, gas-phase thermolysis experiment to be carried out.

\subsection{References}

1 Dopplet, P. Coord. Chem. Rev. 1998, 1785.

2 (a) Leskela, M.; Ritala, M. Angew. Chem., Int. Ed. 2003, 42, 5548. (b) Ritala, M.; Leskela, M. In Handbook of Thin Film Materials; Nalwa, H. S., Ed.; Atomic Layer Deposition, Chapter 2; Academic Press: San Diego, CA, 2001; Vol. 1; p. 103.

32005 Edition of the International Technology Roadmap for Semiconductors; http://www.itrs.net/Links/2005ITRS/Home2005.htm.

4 (a) Kim, J. Y.; Kim, Y.-G.; Stickney, J. L. J. Electrochem. Soc. 2007, 154 , 260. (b) Park, K.-H.; Bradley, A. Z.; Thompson, J. S.; Marshall, W. J. Inorg. Chem. 2006, 45, 
8480. (c) Jezewski C.; Lanford W.A.; Wiegand C.J.; Singh J.P.; Wang P.I.; Senkevich J.J.; Lu T.M. J. Electrochem. Soc. 2005, 152, 60. (d) Mane A.U.; Shivashankar, S.A. Mat. Sci. Semicon. Proc. 2004, 7, 343. (e) Niskanen A.; Rahtu A.; Sajavaara T.; Arstila K.; Ritala M.; Leskela M. J. Electrochem. Soc. 2005, 152, 25 .

5 (a) Chen, T. Y.; Omnés, L.; Vaisserman, J.; Doppelt, P. Inorg. Chim. Acta 2004, 357, 1299. (b) Bollmann, D.; Merkel, R.; Klumpp, A. Microelectron. Eng. 1997, 37- 38, 105. (c) Lagalante, A. F.; Hansen, B. N.; Bruno, T. J.; Sievers, R. E. Inorg. Chem. 1995, 34, 5781. (d) Wenzel, T. J.; Williams, E. J.; Haltiwanger, R. C.; Sievers, R. E. Polyhedron 1985, 4, 369.

6 Lay, E.; Song, Y.-H.; Chiu, Y.-C.; Lin, Y.-M.; Chi, Y.; Carty, A. J. Inorg. Chem. 2005, 44, 7226.

7 Park, K.-H.; Marshall, W. J. J. Am. Chem. Soc. 2005, 127, 9330.

8 (a) Li, Z.W.; Rahtu, A.; Gordon, R.G. J. Electrochem. Soc. 2006, 153, 787. (b) Li, Z.; Barry, S. T.; Gordon, R. G. Inorg. Chem. 2005, 44, 1728. (c) Lim, B. S.; Rahtu, A.; Gordon, R. G. Nat. Mater. 2003, 2, 749. (d) Lim, B. S.; Rahtu, A.; Park, J.; Gordon, R. G. Inorg. Chem. 2003, 42, 7951.

9 Jiang, X.; Bollinger, J. C.; Baik, M.-H.; Lee, D. Chem. Commun. 2005, 1043.

10 Rowley, C. N.; DiLabio, G. A.; Barry, S. T. Inorg. Chem. 2005, 44, 1983.

11 Kenney, A. P.; Yap, G. P. A.; Richeson, D. S.; Barry, S. T. Inorg. Chem. 2005, 44, 2926.

12 Brazeau, A. L.; Wang, Z.; Rowley, C. N.; Barry, S. T. Inorg. Chem. 2006, 45, 2276.

13 Cotton, F. A.; Wilkinson, G; Murillo, C. A; Bochmann, M. Advanced Inorganic Chemistry; 6th ed.; Wiley-Interscience: New York, 1999, p 861.

14 Tsuda, T.; Wantanabe, K.; Miyata, K.; Yamamoto, H.; Saegusa, T. Inorg. Chem. 1981, 20, 2728.

15 Shimokawa, C.; Tachi, Y.; Nishiwaki, N.; Ariga, M.; Itoh, S. Bull. Chem. Soc. Jpn. 2006, 79, 118.

16 (a) Chen, T.-Y.; Vaissermann, J.; Ruiz, E.; Sénateur, J. P.; Doppelt, P. Chem. Mater. 2001, 13, 3993. (b) Chi, K. M.; Shin, H.-K.; Hampden-Smith, M. J.; Duesler, E. N. Polyhedron 1991, 19, 2293. 
17 Kaloyeros A. E.; Eisenbraun E. Annu. Rev. Mater. Sci. 2000, 30, 363.

18 Foley, S. R.; Yap, G. P. A.; Richeson, D. S. Chem. Commun. 2000, 1515 and references therein.

19 Sheldrick, G. M. SHELXTL 6.12; Siemens XRD: Madison, WI, 2001. 
3 Chapter: Gas-Phase Thermolysis of a Guanidinate Precursor of Copper Studied by Matrix Isolation, Time-of-Flight Mass Spectrometry and Computational Chemistry

Modified from original manuscript published as:

Coyle, J. P. ${ }^{1,2}$; Johnson, P. A. ${ }^{1,3}$; DiLabio, G. A. ${ }^{3}$; Barry, S. T. ${ }^{1}$; Muller, J. ${ }^{2}$ "Gas-Phase Thermolysis of a Guanidinate Precursor of Copper Studied by Matrix Isolation, Time-ofFlight Mass Spectrometry, and Computational Chemistry" Inorg. Chem. 2010, 49, 28442850

${ }^{1}$ Department of Chemistry, Carleton University, 1125 Colonel By Drive, Ottawa, Ontario, Canada K1S 5B6.

${ }^{2}$ Department of Chemistry, University of Saskatchewan, 110 Science Place, Saskatoon, Saskatchewan, S7N 5C9.

${ }^{3}$ National Institute for Nanotechnology, National Research Council of Canada, 11421

Saskatchewan Drive, Edmonton, Alberta, Canada T6G 2M9

\subsection{Abstract}

The fragmentation of the copper(I) guanidinate $\left[\mathrm{Me}_{2} \mathrm{NC}(\mathrm{NiPr})_{2} \mathrm{Cu}\right]_{2}(\mathbf{1})$ has been investigated with time-of-flight mass spectrometry (TOF MS), matrix-isolation FTIR spectroscopy (MI FTIR spectroscopy) and DFT calculations. Gas-phase thermolyses of $\mathbf{1}$ were performed in the temperature range of $100-800^{\circ} \mathrm{C}$. TOF MS and MI FTIR gave consistent results, showing that precursor $\mathbf{1}$ starts to fragment at oven temperatures above $150{ }^{\circ} \mathrm{C}$, with a close to complete fragmentation at $260^{\circ} \mathrm{C}$. Precursor $\mathbf{1}$ thermally fragments to $\mathrm{Cu}_{(\mathrm{s})}, \mathrm{H}_{2}(\mathrm{~g})$ and the oxidized guanidine $\mathrm{Me}_{2} \mathrm{NC}(=\mathrm{Ni} \mathrm{Pr})\left(\mathrm{N}=\mathrm{CMe}_{2}\right)(3)$. In TOF MS experiment 3 was clearly indentified by its molecular ion at $169.2 \mathrm{u}$. Whereas $\mathrm{H}_{2}{ }^{+}$was detected, atomic $\mathrm{Cu}$ was not found in gas-phase thermolysis. In addition the guanidine $\mathrm{Me}_{2} \mathrm{NC}(\mathrm{N} i \mathrm{Pr})(\mathrm{NH} i \mathrm{Pr})(2)$ was detected as a minor component among the thermolysis products. MI thermolysis experiments with precursor $\mathbf{1}$ were performed and species evolving from the thermolysis oven were trapped in solid argon at $20 \mathrm{~K}$. These species were characterized by FTIR spectroscopy. The most indicative feature of the 
resulting spectra from thermolysis above $150{ }^{\circ} \mathrm{C}$ was a set of intense and structured peaks between 1600 and $1700 \mathrm{~cm}^{-1}$, an area where precursor 1 does not have any absorbances. The guanidine $\mathbf{2}$ was matrix-isolated and a comparison of its FTIR spectrum with the spectra of the thermolysis of $\mathbf{1}$ indicated that species $\mathbf{2}$ was among the thermolysis products. However, the main IR bands in the range of 1600 and $1700 \mathrm{~cm}^{-1}$ appeared at $1687.9,1668.9,1635.1$, and $1626.6 \mathrm{~cm}^{-1}$ and were not caused by species $\mathbf{2}$. The oxidized guanidine $\mathbf{3}$ was synthesized for the first time and characterized by ${ }^{1} \mathrm{H}$ NMR and FTIR spectroscopy. A comparison of an FTIR spectrum of matrix isolated $\mathbf{3}$ with spectra of the thermolysis of 1 revealed that the main IR bands in the range of 1600 and $1700 \mathrm{~cm}^{-1}$ a due to the presence of 3. Based on DFT calculations at the BLYP/6-31G(d) level, the four bands could be assigned to $\mathrm{C}=\mathrm{N}$ stretching modes of one isomer of $\mathbf{3}$. The isomers exhibit the $\mathrm{NMe}_{2}$ group cis or trans to the $i$ Pr group, with cis-3 being significantly less stable than trans-3. At higher temperature secondary thermal fragments had been observed. For example at $700{ }^{\circ} \mathrm{C}$, TOF MS and MI FTIR data showed that species 2 and 3, both eliminate $\mathrm{HNMe}_{2}$ to give the carbodiimides $i \operatorname{PrNCN} i \operatorname{Pr}(\mathrm{CDI})$ and $i \operatorname{PrNCN}\left[\mathrm{C}\left(=\mathrm{CH}_{2}\right) \mathrm{Me}\right](4)$, respectively. A DFT study of the decomposition of compound 1 was undertaken at the B3LYP/6-31+G(d,p) level of theory employing dispersioncorrecting potentials (DCPs). The DFT study rationalized both carbodiimide deinsertion and $\beta$-hydrogen elimination as exergonic decomposition pathways at $260^{\circ} \mathrm{C}(\Delta \mathrm{G}=-44.4$ $\mathrm{kcal} / \mathrm{mol}$ in both cases), but experiment showed $\beta$-hydrogen elimination to be the favourable route. 


\subsection{Introduction}

Thin films of copper metal are of utmost interest in microelectronics, specifically for use as an interconnect material. Several copper(I) precursors have been reported for chemical vapour deposition (CVD) and atomic layer deposition (ALD), including $\beta$ diketonates, ${ }^{1-4} \beta$-diketiminates, ${ }^{5}$ amidinates, ${ }^{6-9}$ and guanidinates ${ }^{10}$.

The guanidinate dimer of copper(I), $\left[\mathrm{Me}_{2} \mathrm{NC}(\mathrm{N} i \mathrm{Pr})_{2} \mathrm{Cu}\right]_{2}(\mathbf{1})$, deposits copper in a CVD process above $225^{\circ} \mathrm{C}$ as a nanocrystalline film; ${ }^{10}$ similar reactivity is known for the acetamidinatocopper(I) dimer. ${ }^{9}$ An interesting aspect of the deposition is that these precursors act as single source precursors for copper, meaning that they do not require a second, reducing precursor to produce copper metal. The solution-phase thermolysis of the guanidinate compound $\mathbf{1}$ showed the production of diisopropylcarbodiimide (CDI), and thus we suggested a mechanism whereby CDI deinserted from the ligand to produce a copper amide intermediate, $\mathrm{Cu}_{2}\left(\mathrm{NMe}_{2}\right)_{2}$, which then eliminated an imine and an amine to produce metallic copper (Figure 3.1). ${ }^{10}$

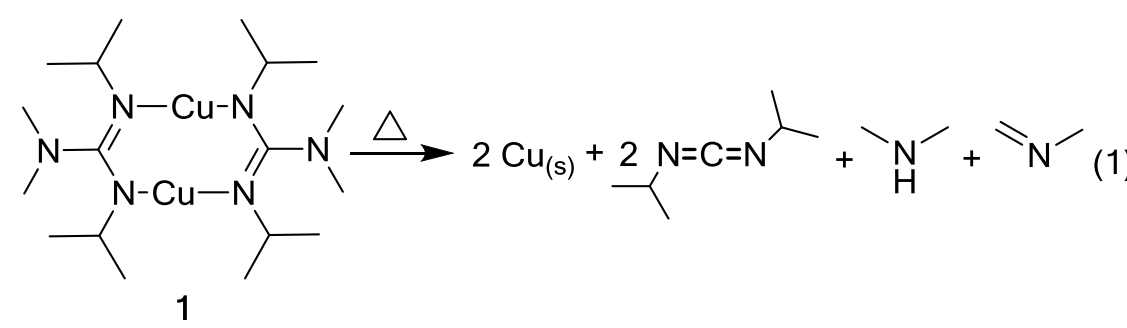

Figure 3.1 Decomposition of a Copper Guanidinate via CDI Deinsertion

We wanted to further investigate this mechanism, particularly in light of a recent report of $\beta$-hydrogen elimination as a potential thermolysis route. ${ }^{11}$ In addition, we recently unraveled the fragmentation of an alumina ALD precursor, the aluminum guanidinate $\left[\mathrm{Me}_{2} \mathrm{NC}(\mathrm{NiPr})_{2}\right] \mathrm{Al}\left(\mathrm{NMe}_{2}\right)_{2}$, by time-of-flight mass spectrometry (TOF MS) and matrix-isolation FTIR spectroscopy (MI FTIR spectroscopy). ${ }^{12}$ Using these 
techniques, we have shown that this precursor eliminates CDI to give the monomeric aluminum amide $\mathrm{Al}\left(\mathrm{NMe}_{2}\right)_{3}$ above $300{ }^{\circ} \mathrm{C} .{ }^{12}$ Thus, we employed these techniques to further investigate the gas-phase thermolyses of the copper precursor $\mathbf{1}$ in the temperature range of $100-800^{\circ} \mathrm{C}$. We report on results of TOF MS, MI FTIR spectroscopy, and DFT calculations.

\subsection{Results and Discussion}

Since Pimentel's pioneering work on matrix-isolation spectroscopy, ${ }^{13,14}$ this technique matured into a well-known method to trap and identify reactive intermediates. Mass spectrometry is a highly sensitive method to investigate gas-phase species and it has been used to elucidate thermal fragmentations of precursors. Recently, we combined both techniques in a unique apparatus in which the thermolysis of a CVD precursor can be investigated using MI FTIR spectroscopy and TOF MS. ${ }^{12}$ The facility was designed in such a way that a series of experiments can be performed using one method, which can then be followed by a similar series of experiments on the same precursor sample and carrier gas supply using the second method. Consequently, MI FTIR and TOF MS data are collected consecutively, but the conditions in both series of experiments are kept as similar as possible so that their data complement one another.

TOF Mass Spectrometry. The mass spectrum at the bottom of Figure 3.2 shows the typical ion pattern of precursor $\mathbf{1}$ in the absence of any thermal decomposition; all fragments of the molecular ion were caused by ion impact ionization at $70 \mathrm{eV}$ of the precursor $\mathbf{1}$. An oven temperature of $130^{\circ} \mathrm{C}$ was used to avoid any build-up of $\mathbf{1}$ in the apparatus through condensation. The highest detectable masses in this spectrum are due to $\mathrm{M}^{+}$of $\mathbf{1}$ (enlargement in Figure 3.2); its isotopic pattern agrees well with the calculated 
one (see Experimental Section). It should be noted that the ion at $465.3 \mathrm{u}$ is not part of the isotopic pattern of $\mathbf{1}^{+}$, but results from the loss of one $\mathrm{H}$ atom $\left(\mathbf{1}^{+}-\mathrm{H}\right)$. When the oven temperature was increased to $260{ }^{\circ} \mathrm{C}$ (Figure 3.2 top spectrum), $\mathbf{1}^{+}$could no longer be detected $^{15}$ and the highest mass appeared at $171.2 \mathrm{u}$ followed by a mass of higher intensity at $169.2 \mathrm{u}$. The mass of $171.2 \mathrm{u}$ matches with the empirical formula of $\mathrm{C}_{9} \mathrm{H}_{21} \mathrm{~N}_{3}{ }^{+}$ (calc. mass of $171.17 \mathrm{u}$ ) and can be interpreted as the cation of the protonated ligand $\mathbf{2}$ (Figure 3.3). The peak at $169.2 \mathrm{u}$ fits well to $\mathrm{C}_{9} \mathrm{H}_{19} \mathrm{~N}_{3}{ }^{+}$(calc. mass of $169.16 \mathrm{u}$ ) and can be interpreted as dehydrogenated $\mathbf{2}$. From the numerous possibilities to assign a structural formula to the mass of $169.2 \mathrm{u}$, we assign structure $\mathbf{3}$ (Figure 3.3) for reasons that will become apparent in the following discussion.

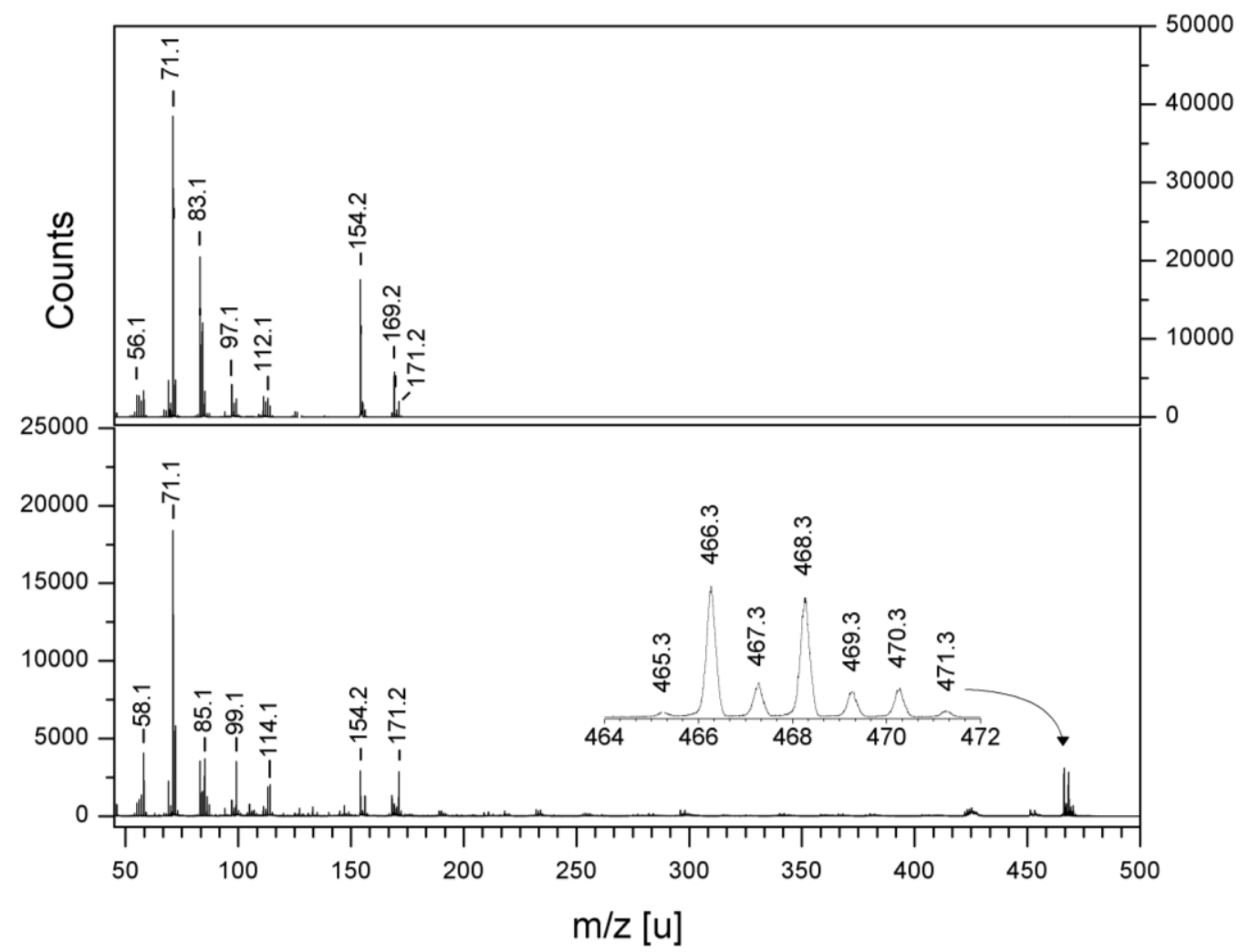

Figure 3.2 Overview MS of the copper guanidinate 1 with thermolysis oven at $130{ }^{\circ} \mathrm{C}$ (no thermal fragmentation; bottom spectrum; $\mathrm{M}^{+}$of 1 enlarged) and at $260^{\circ} \mathrm{C}$ (complete thermal fragmentation of $\mathbf{1}$; top spectrum). 


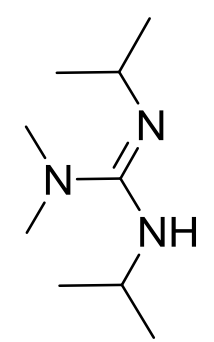

2 (171.17 u)

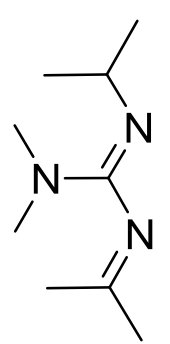

3 (169.16 u)

Figure 3.3 Protonated and oxidized ligand with calculated masses.

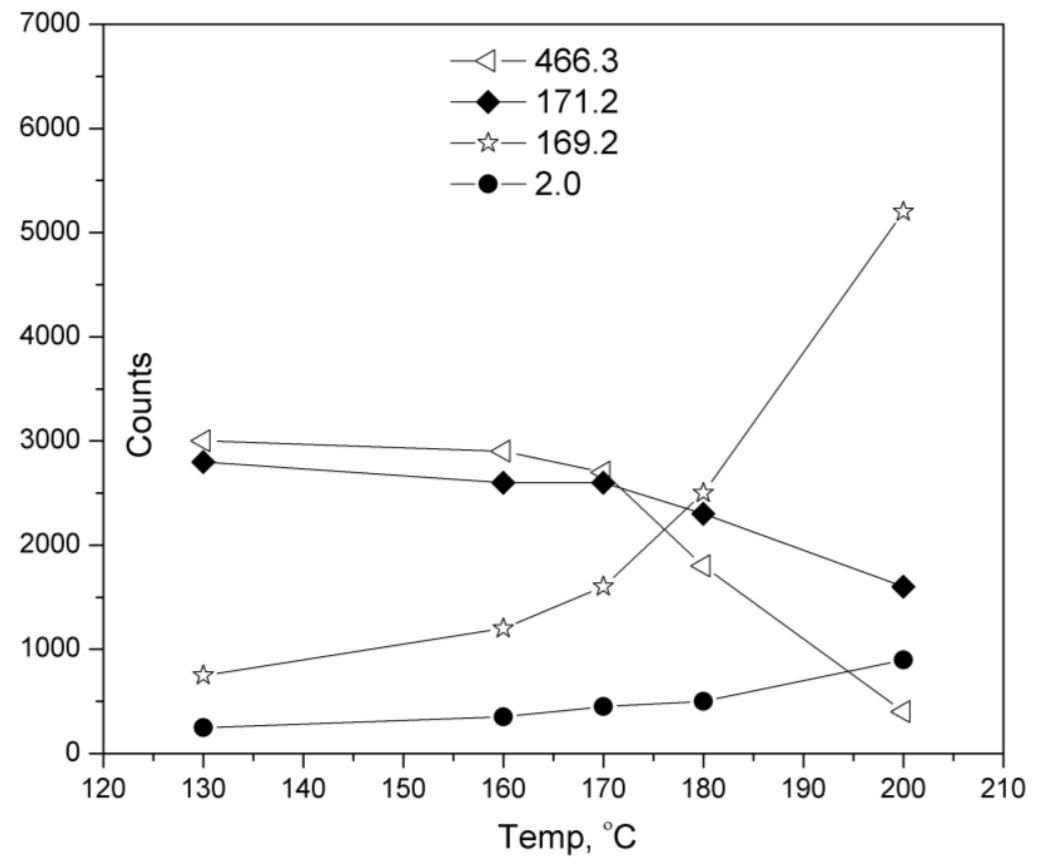

Figure 3.4 Temperature dependence of intensities of selected ions arising from the thermolysis of $\mathbf{1}\left[\mathrm{m} / \mathrm{z}=466.3\left(\mathbf{1}^{+}\right), 171.2\left(\mathbf{2}^{+}\right), 169.2\left(\mathbf{3}^{+}\right), 2.0\left(\mathrm{H}_{2}^{+}\right) \mathrm{u}\right]$.

Figure 3.4 shows how signal intensities of selected ions changed in the temperature range of $130-200{ }^{\circ} \mathrm{C}$. The signal intensity of $\mathrm{M}^{+}(466.3 \mathrm{u})$ of the precursor 1 decrease slightly from 160 to $170{ }^{\circ} \mathrm{C}$, followed by a steeper decrease at temperatures above $170{ }^{\circ} \mathrm{C}$. A comparable change of intensities was observed for the signal of $2^{+}$ $(171.2 \mathrm{u})$. At the same time, the signal of $\mathbf{3}^{+}(169.2 \mathrm{u})$ showed a significant increase in intensity, which indicates that $\mathbf{3}^{+}$is an ion of a thermolysis fragment of precursor $\mathbf{1}$. 
In addition to the identification of the peak at $169.2 \mathrm{u}$, which we assign to the structural formula 3 (Figure 3.3), there is a significant increase of the signal intensity at $2.0 \mathrm{u}$, which is due to the presence of $\mathrm{H}_{2}{ }^{+}$. Copper atoms were not detected in the gas phase; however, after a long series of thermolyses, the thermolysis oven was inspected and had a copper color at the oven orifice. This is not surprising, as it was shown that $\mathbf{1}$ is a precursor for the thermal deposition of $\mathrm{Cu}^{10}{ }^{10}$ Based on these data, we propose that the thermolysis of precursor 1 can be described as shown in Figure 3.5. We performed DFT calculations at the $\mathrm{B} 3 \mathrm{LYP} / 6-31+\mathrm{G}(\mathrm{d}, \mathrm{p})$ level of theory employing dispersion-correcting potentials (DCPs), which showed that the production of $\mathbf{3}$ through $\beta$-hydrogen elimination is thermodynamically favorable at $260{ }^{\circ} \mathrm{C}(\Delta \mathrm{G}=-44.4 \mathrm{kcal} / \mathrm{mol}) .{ }^{16}$

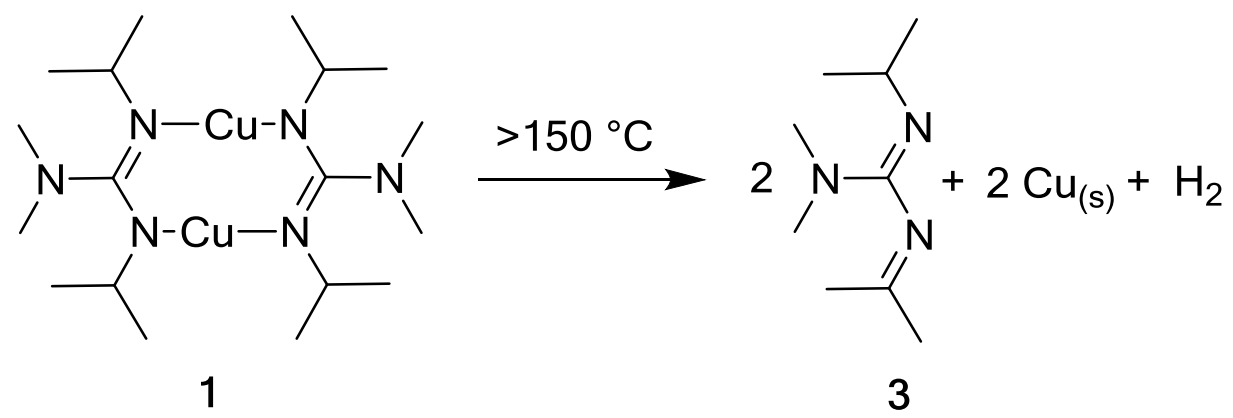

Figure 3.5 Decomposition of a Copper Guanidinate via $\beta$-hydride Elimination.

Compound $\mathbf{3}$ is the formal dehydrogenated product of the protonated ligand $\mathbf{2}$ and it is feasible that $\mathbf{2}$, instead of $\mathbf{3}$, is the primary thermolysis product of the precursor $\mathbf{1}$. Species $\mathbf{2}$ could further decompose to give the detected species $\mathbf{3}$. Therefore, an independent thermolysis series with the protonated ligand $\mathbf{2}$ as a starting compound was undertaken. This study revealed that, for temperatures of up to $800{ }^{\circ} \mathrm{C}$ in the presence of a copper metal surface, species $\mathbf{2}$ does not eliminate $\mathrm{H}_{2}$ to give $\mathbf{3}$. In all mass spectra of $\mathbf{2}$ 
the signal at $169.2 \mathrm{u}$ appeared only with negligible intensities compared to that at $171.2 \mathrm{u}$ $\left(2^{+}\right)$

When considering Figure 3.5, it might seem possible for the reaction to proceed by disproportion of $\mathrm{Cu}(\mathrm{I})$ to $\mathrm{Cu}(\mathrm{II})$ and $\mathrm{Cu}(0)$, giving an undetected (and perhaps unstable and transient) copper(II) species. This has been suggested for the amidinate depositing copper on silicon dioxide. ${ }^{17}$ However, our DFT calculations (at the B3LYP/6-31+G(d,p) level of theory with DCPs and making the same assumptions as previous) show this to be unlikely for a gas phase species: it would have a $\Delta \mathrm{G}$ of $10.9 \mathrm{kcal} / \mathrm{mol}$ (compared to -44.4 $\mathrm{kcal} / \mathrm{mol}$ for $\beta$-hydrogen elimination from a copper(I) species). As well, the FTIR spectra throughout the series of thermolysis experiments did not show any peaks that could not be assigned specifically to the reactants and products of Figure 3.5. Specifically, the region from $1500-1800 \mathrm{~cm}^{-1}$ (where a $\mathrm{C}=\mathrm{N}$ stretching mode for a $\mathrm{Cu}(\mathrm{II})$ guanidinate species would appear) contained no unassigned peaks. Finally, similar mechanistic work for surface-bound copper species on a nickel surface resulting from the thermolysis of a copper amidinate similarly found no experimental evidence for a copper(II) species. ${ }^{18}$ The sum of the evidence suggests that we do not see a copper(II) species in these experiments.

Considering Figure 3.5, it also seems possible that the decomposition might proceed by a process involving homolytic scission of the $\mathrm{Cu}-\mathrm{N}$ bonds to produce free radical intermediates, which may then disproportionate to form $\mathbf{2}$ and $\mathbf{3}$. DFT calculations at the B3LYP/6-31+G(d,p) level of theory with DCPs indicate that this process is endergonic by $33.6 \mathrm{kcal} / \mathrm{mol}$, and therefore also unlikely. The thermolysis of copper(I) alkyls has been studied by Whitesides et al. ${ }^{19-21}$ Their study of n-butylcopper(I) 
compounds revealed a $\beta$-hydride elimination pathway. ${ }^{19}$ The decomposition of 1propenylcopper(I) was also found not to proceed by a pathway involving free radical intermediates despite the stability of the vinyl radical. ${ }^{20}$ However, neophyl(tri-nbutylphosphine)copper(I) was found to produce the neophyl radical, which is known to rearrange to an exceptionally stable radical. ${ }^{21}$ These studies have shown that free radicals are unlikely intermediates in the thermolysis of copper(I) alkyls unless the free radical itself is very stable. Copper(I) amides have been found to decompose via $\beta$-hydride elimination and to be more stable than their corresponding alkyls. ${ }^{22}$ On this basis, it seems unlikely that a mechanism involving free radical intermediates is involved in the decomposition of $\mathbf{1}$.

Alternatively, one might expect the thermolysis reaction as illustrated in Figure 3.5 to be initiated by the decomposition of the dimer $\mathbf{1}$ to form two copper guanidinate monomers, which subsequently decompose. Using DFT calculations, we predict that this pathway is very unlikely at $260^{\circ} \mathrm{C}$, viz. $\Delta \mathrm{G}=66.7 \mathrm{kcal} / \mathrm{mol}$ for the formation of two copper(I) guanidinate monomers. In addition, there was no evidence of the monomer $\left[\mathrm{Me}_{2} \mathrm{NC}(\mathrm{NiPr})_{2} \mathrm{Cu}\right]$ in the thermolysis series of the dimer $\mathbf{1}$; the measured intensities of signals showing fragments with one $\mathrm{Cu}$ atom indicated that they were caused by the impact ionization of $\mathbf{1}$ alone.

MI FTIR Spectroscopy. The thermal decomposition of precursor 1 was also investigated with MI FTIR spectroscopy, using similar experimental conditions as for the TOF MS experiments discussed above. At oven temperatures of up to $150^{\circ} \mathrm{C}$, IR spectra did not change, thus indicating that precursor 1 did not decompose (see Supporting Information, Figure 3.11). At temperatures of $160{ }^{\circ} \mathrm{C}$, new IR peaks indicated the onset of 
the thermolysis. The most indicative feature of the resulting spectrum was a set of intense and structured peaks between 1600 and $1700 \mathrm{~cm}^{-1}$, an area where precursor 1 does not have any absorbances. Spectrum B of Figure 3.6 shows the result of a thermolysis of $\mathbf{1}$ at $260{ }^{\circ} \mathrm{C}$. The investigation of $\mathbf{1}$ by TOF MS revealed that the protonated ligand $\mathbf{2}$ was a product in the gas phase. In order to find out if $\mathbf{2}$ was also present in the matrices, a MI FTIR spectrum of the synthesized sample of 2 was measured (Figure 3.6, spectrum A). The comparison of spectrum A with B indicates that $\mathbf{2}$ was one of the thermolysis products of 1 . Species 2 has its most intense IR band at $1646.0 \mathrm{~cm}^{-1}$, which appears as a shoulder of the intense features between 1600 and $1700 \mathrm{~cm}^{-1}$ in spectrum B (see dotted line in Figure 3.6). The TOF MS experiments clearly indicated that $\mathbf{3}$ was one of the key fragments of the thermolysis of the copper precursor 1 (Figure 3.5). Similarly to the identification of $\mathbf{2}$ as a thermolysis product, we intended to identify $\mathbf{3}$ through comparison with FTIR spectra. To our surprise, species $\mathbf{3}$ was unknown in the literature, and consequently needed to be synthesized. 


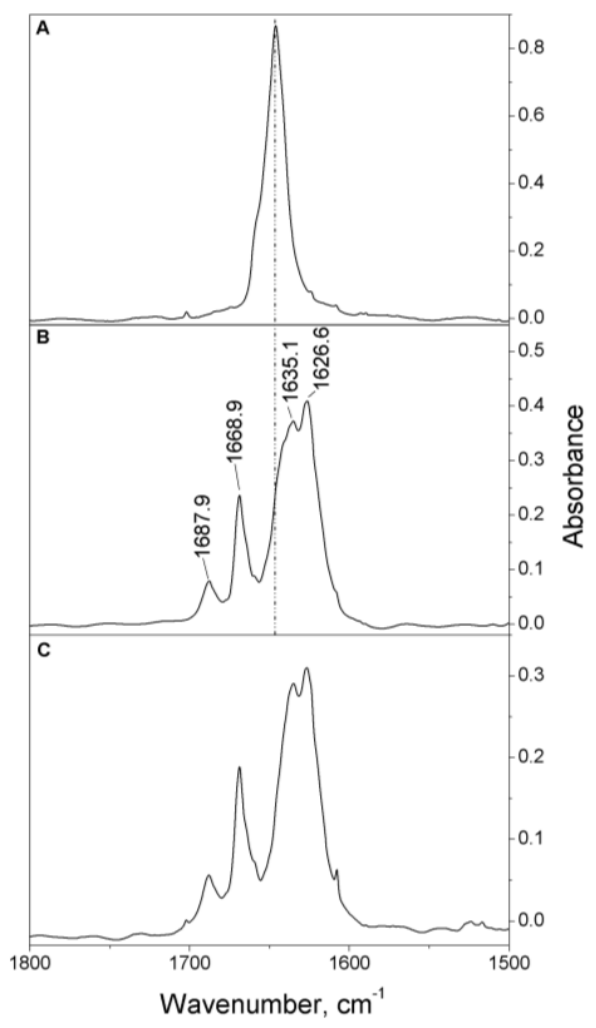

Figure 3.6 Measured IR spectra of copper guanidinate 1 and its thermolysis products $(x$ axis, $1500-1800 \mathrm{~cm}^{-1} ; y$ axis, absorbance). Spectrum A: 2 in solid argon (thermolysis oven at $260^{\circ} \mathrm{C}$ ); see Figure 3.13 for a complete IR spectrum. Spectrum B: results of a thermolysis of complex 1 at $260{ }^{\circ} \mathrm{C}$; see Figure 3.12 for a complete IR spectrum. Spectrum C: 3 in solid argon (thermolysis oven at $260{ }^{\circ} \mathrm{C}$ ); residual amounts of $\mathbf{2}$ have been electronically eliminated; see Figure 3.14 for a complete IR spectrum.

Based on a known method to synthesize imines from secondary amines, ${ }^{23} \mathbf{2}$ was oxidized using 2 equiv. of $\mathrm{CuBr}_{2}$ and 2 equiv. of $\mathrm{LiO} t \mathrm{Bu}$ to give species 3 . Species 3 was first characterized by ${ }^{1} \mathrm{H}$ NMR spectroscopy, which showed that in addition to the target compound $\mathbf{3}$, small amounts of the starting compound $\mathbf{2}$ were present. In particular, the two singlets of equal intensity at 1.43 and $1.72 \mathrm{ppm}$ (in $\mathrm{C}_{6} \mathrm{D}_{6}$ ) clearly revealed the two inequivalent Me groups of the $\mathrm{N}=\mathrm{CMe}_{2}$ moiety of $\mathbf{3}$ (Figure 3.3). This compound proved very difficult to isolate from the parent guanidine and ${ }^{\mathrm{t}} \mathrm{BuOH}$, all of which have very similar boiling points, but the IR was captured in matrix isolation, and matched the 
calculated vibrational frequencies such that we are satisfied of its formulation (Figure 3.7, Figure 3.14 in Supporting Information). We are continuing to try to isolate this material to further our thermolysis studies of this system.

A so-prepared sample was vaporized and trapped in solid argon; spectrum C of Figure 3.6 shows the resulting IR spectrum. All measured IR bands of $\mathbf{3}$ (spectrum C) are present in spectra of the thermolysis of precursor $\mathbf{1}$ (spectrum B), giving strong evidence that 3 is a thermolysis product. The structured IR band in the range of 1600 and $1700 \mathrm{~cm}^{-}$ ${ }^{1}$ with four maxima at $1687.9,1668.9,1635.1$, and $1626.6 \mathrm{~cm}^{-1}$ must be caused by $\mathrm{C}=\mathrm{N}$ stretching modes. As compound $\mathbf{3}$ exhibits two $\mathrm{C}=\mathrm{N}$ bonds, two $\mathrm{C}=\mathrm{N}$ stretching modes are expected in the IR range. The fact that the IR absorbance between 1600 and $1700 \mathrm{~cm}^{-}$ ${ }^{1}$ shows more than two bands, suggests that perhaps different isomers of $\mathbf{3}$ are present upon trapping. In order to better understand the structure of the IR band in the $\mathrm{C}=\mathrm{N}$ stretching region, DFT calculations at the BLYP/6-31G(d) level of theory were performed. The lowest energy structure of $\mathbf{3}$ (Figure 3.7) has a trans orientation of the $\mathrm{Me}_{2} \mathrm{~N}$ and the $i \operatorname{Pr}$ group, and was the only isomer that appeared in an appreciable amount (Figure 3.7). A second isomer with a cis orientation of the $\mathrm{MeN}_{2}$ and the $i \mathrm{Pr}$ group is likely to be trapped, as well, in the solid matrix.

The calculated frequencies of the $\mathrm{C}=\mathrm{N}$ stretches of trans-3 fit very well to the experimental data: the calculated (BLYP/6-31G(d)) $)^{24} /$ experimental frequencies for $v(\mathrm{C} 6=\mathrm{N} 1)$ were $1652.9 / 1668.9$ and for $v(\mathrm{C} 1=\mathrm{N} 2)$ they were $1598.8 / 1635.1 \mathrm{~cm}^{-1}$ (Figure 3.7). The calculated intensity pattern (ca. $3: 1$ ) also fits well to the measured peak ratios: the two IR bands at lower frequencies are more intense than those at higher frequencies. However, these assignments are only tentative. For an unambiguous assignment of 
experimental IR bands, a complete vibrational analysis of $\mathbf{3}$ would be necessary. Such an analysis would necessarily be based on experimental IR data of different isotopomers of 3, which would require extended synthetic work first. However, such an investigation is beyond the scope of our present work.
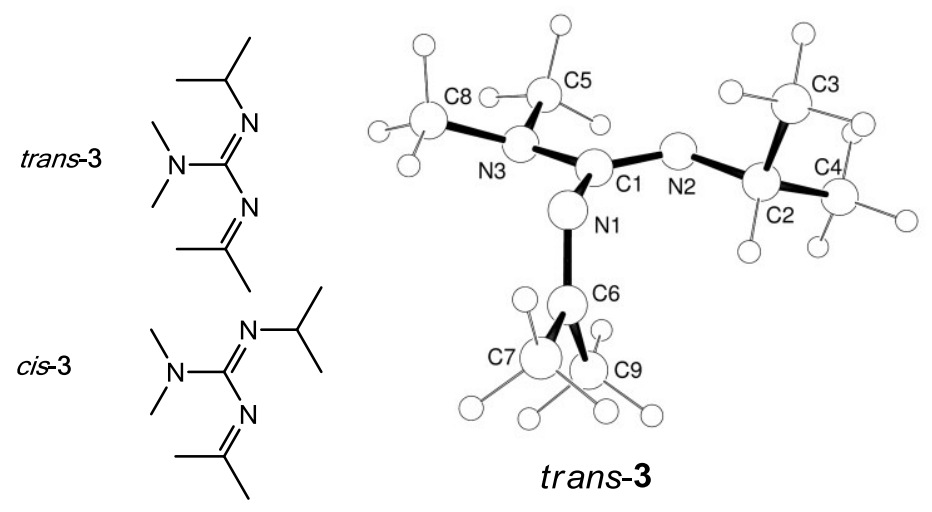

Figure 3.7 Possible isomers of $\mathbf{3}$ and the optimized structure for trans-3 obtained with B3LYP/6-31+G(d,p)-DCP.

It is interesting that the thermolysis of the copper(I) guanidinate at these temperatures found no indication of CDI, as was previously found in sealed NMR tube thermolysis experiments. ${ }^{11}$ An extensive DFT study of the decomposition of compound 1 was undertaken, and this helps rationalize these two different thermolysis pathways. These calculations were undertaken at the B3LYP/6-31+G(d,p) level of theory employing DCPs.

DFT Calculations. The calculated mechanism showed that 1 undergoes a shift of a nitrogen atom of one of the guanidinate ligands to a $\mu_{2}$ bonding arrangement, as well as a rotation of one of this ligand's isopropyl groups such that the hydrogen on this group is oriented toward one copper centre (Figure 3.8, path A). This step is calculated to be uphill in free energy by $22.5 \mathrm{kcal} / \mathrm{mol}$. The resulting species $\mathbf{A 1}$ is the branching point between $\beta$-hydrogen elimination (path B) and CDI elimination (path C). 


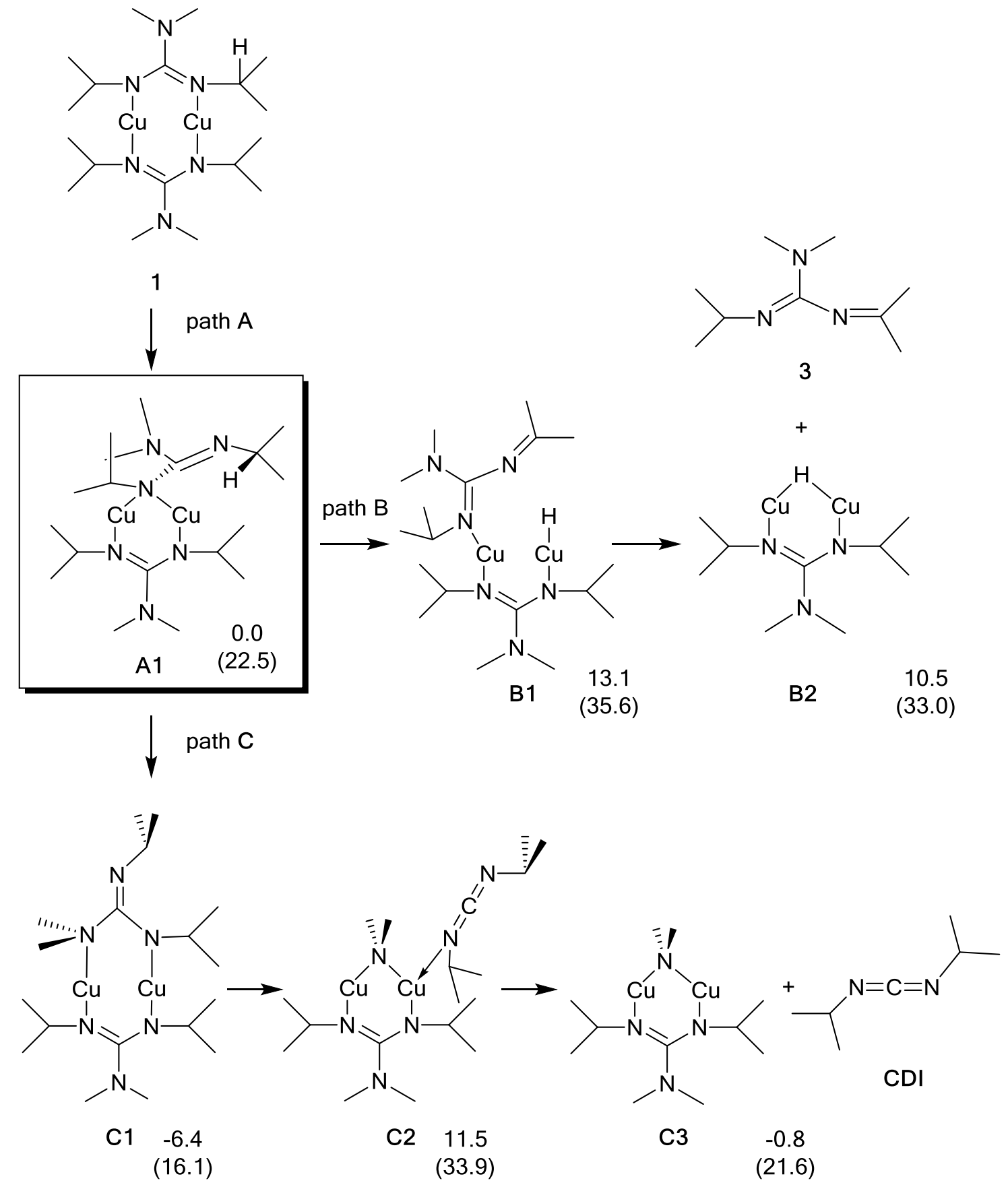

Figure 3.8 Calculated fragmentation pathways for precursor 1. Free energies relative to $\mathbf{A 1}$ for the reaction steps are shown (values in parentheses are relative to species 1)

As shown in path B of Figure 3.8, compound 3 can be formed through elimination of the isopropyl methine proton (located in a $\delta$-position) to form a copper hydride (B1), 
which is an uphill process by $13.1 \mathrm{kcal} / \mathrm{mol}$ (free energy). The hydride $\mathbf{B} 1$ eliminates $\mathbf{3}$ in a barrierless process. The path from $\mathbf{A} \mathbf{1}$ to the products $\mathbf{B} \mathbf{2}$ and $\mathbf{3}$ is endergonic by 10.5 $\mathrm{kcal} / \mathrm{mol}(33.0 \mathrm{kcal} / \mathrm{mol}$ from $\mathbf{1})$.

Instead of undergoing $\beta$-hydrogen elimination, $\mathbf{A 1}$ can undergo a shift of the $\mu_{2^{-}}$ nitrogen to coordinate one copper atom, with a concurrent rotation about the $\mathrm{Cu}-\mathrm{N}$ bond to allow the exocyclic dimethylamido to coordinate to the other copper atom to form species $\mathbf{C 1}$. This step is thermodynamically downhill by $6.4 \mathrm{kcal} / \mathrm{mol}$. Compound $\mathbf{C} 1$ can then eliminate the dimethylamido moiety to give the copper compound $\mathbf{C 2}$, which further reacts to form $\mathbf{C 3}$ and free CDI. Energetically, the process from $\mathbf{A 1}$ to products $\mathbf{C} 3$ and CDI is exergonic by $0.8 \mathrm{kcal} / \mathrm{mol}$ (but endergonic by $21.6 \mathrm{kcal} / \mathrm{mol}$ from $\mathbf{1}$ ).

These calculations are a good model for the vapour phase decomposition of $\mathbf{1}$ to metallic copper. However, the previously reported thermolysis to eliminate carbodiimide by deinsertion were performed at lower temperatures and in the solution phase. ${ }^{11}$ These calculations also show that carbodiimide deinsertion is reasonable as a decomposition mechanism, as demonstrated by the convincing divergence at species A1.

Fragmentation at higher temperatures. We investigated the fragmentation of the copper precursor $\mathbf{1}$ and the protonated ligand $\mathbf{2}$ at temperatures of up to $800{ }^{\circ} \mathrm{C}$, and new fragments appeared in the thermolyses of both $\mathbf{1}$ and 2. Figure 3.9 shows the results of two series of thermolysis, spectrum A for $\mathbf{2}$ and spectrum B for 1. As shown in Figure 3.9, the amount of species $3(171.2 \mathrm{u})$, which is a key fragment of the thermolysis of precursor 1, decreased with increasing temperature, while only two signals, at 124.1 and $45.0 \mathrm{u}$, showed a significant increase of intensity. The lower mass at $45.0 \mathrm{u}$ fits to $\mathrm{Me}_{2} \mathrm{NH}$ (calculated mass of $45.06 \mathrm{u}$ ) and the one at higher mass fits to the carbodiimide 4 
(calculated mass of $124.10 \mathrm{u}$, Figure 3.10). These data indicate that the thermolysis product 3 eliminates $\mathrm{Me}_{2} \mathrm{NH}$ to give the carbodiimide 4 . This interpretation is consistent with the decomposition of the protonated ligand 2 (Figure 3.9A). The signal for $\mathbf{2}^{+}$ dropped off, whereas signals at 126.1 and $45.0 \mathrm{u}$ rose with increasing temperatures, showing that 2 eliminates $\mathrm{Me}_{2} \mathrm{NH}$ to give the well-known diisopropylcarbodiimide (CDI, Figure 3.10). As species $\mathbf{2}$ is also produced in the thermolysis of $\mathbf{1}$, its signal at $171.2 \mathrm{u}$ decreased (Figure 3.9). The intensity for the signal of CDI (126.1 u) varies over the temperature range but does not show a trend, which might be due to its overall low intensity.

The TOF MS data and their interpretation are consistent with MI FTIR data obtained from high temperature thermolyses. For example, in the thermolysis of $\mathbf{2}$ at 700 ${ }^{\circ} \mathrm{C}$ we unambiguously identified $\mathrm{HNMe}_{2}$ and $\mathrm{CDI}$, trapped in solid argon (Figure 3.15 in Supporting Information). In the thermolysis of $\mathbf{1}, \mathrm{HNMe}_{2}$ was identified, but the unknown carbodiimide $\mathbf{4}$ could not be unambiguously identified, as a required IR spectrum for comparison is unknown in the literature. However, an intense and structured IR band with a maximum at $2127.7 \mathrm{~cm}^{-1}$ clearly indicates the presence of a carbodiimide (Figure 3.16 in Supporting Information). Moreover, an IR band in the typical range of $\mathrm{C}=\mathrm{C}$ valence vibrations at $1649.2 \mathrm{~cm}^{-1}$ supports the proposed structure of $\mathbf{4}$ (see Figure 3.10). Frequency calculations on 4 at the BLYP/6-31G(d) level of theory give a $\mathrm{C}=\mathrm{C}$ stretch at $1639.0 \mathrm{~cm}^{-1}$ and a $\mathrm{N}=\mathrm{C}=\mathrm{N}$ asymmetric stretch at $2164.7 \mathrm{~cm}^{-1}$. 


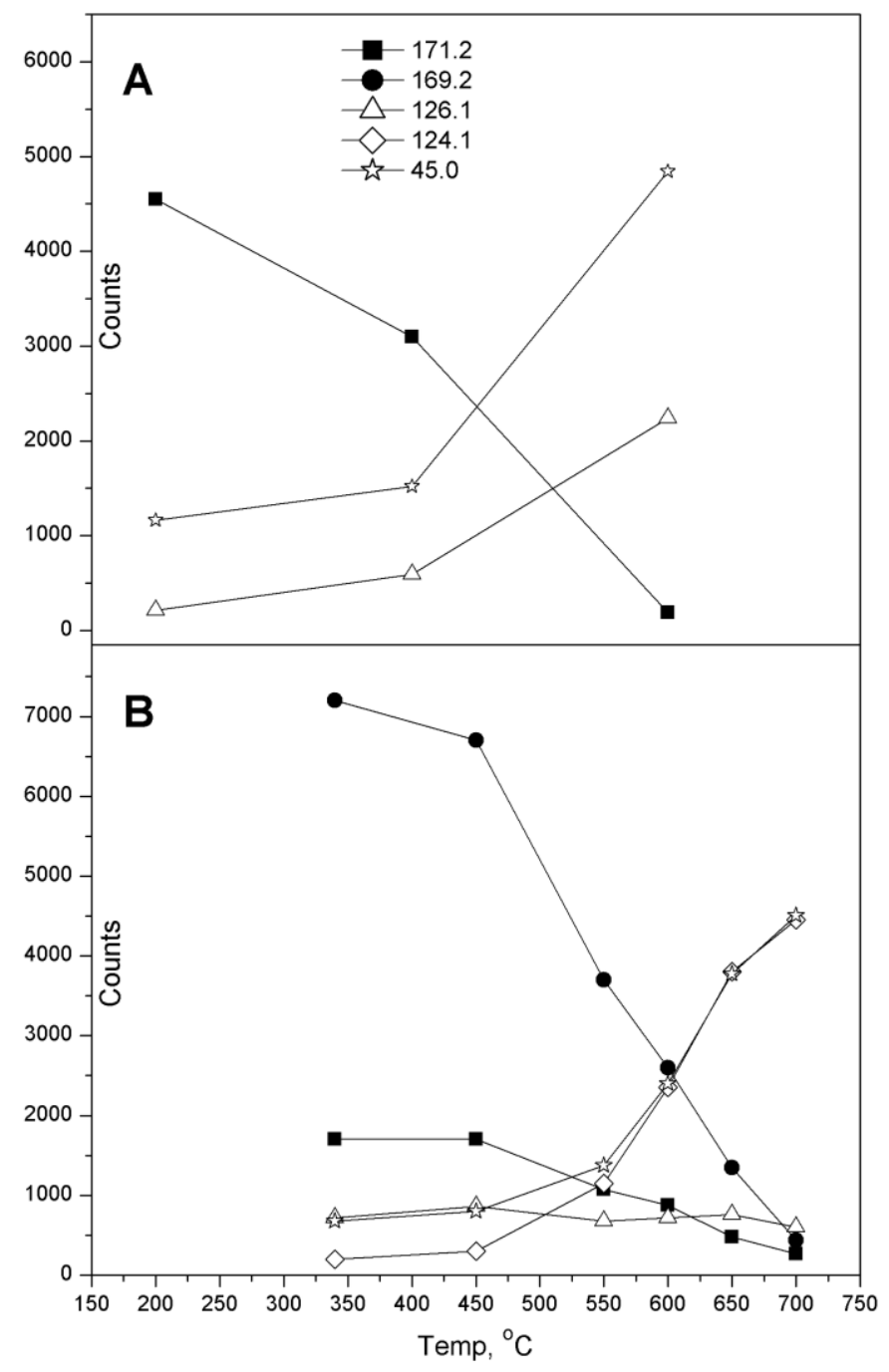

Figure 3.9 Temperature dependences of selected ions of the thermolysis at high temperatures of $\mathbf{1}$ (spectrum B) and $\mathbf{2}$ (spectrum A) $\left[\mathrm{m} / \mathrm{z}=171.2\left(\mathbf{2}^{+}\right), 169.2\left(\mathbf{3}^{+}\right), 126.2\right.$ $\left.\left(4^{+}\right), 124.1\left(\mathbf{C D I}^{+}\right) 45.0\left(\mathrm{HNMe}_{2}{ }^{+}\right) \mathrm{u}\right]$. The intensities of masses at 169.2 and $124.1 \mathrm{u}$ were negligible in spectrum A (counts between 2 and 22 at sweep rates of $8 \times 10^{6}$ ).

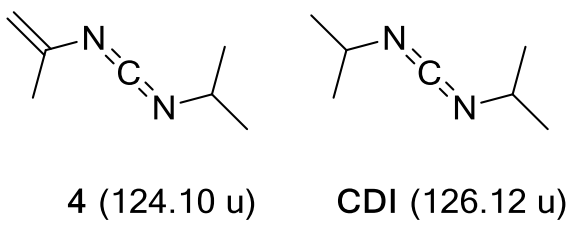

Figure 3.10 Carbodiimides. 


\subsection{Conclusion}

The fragmentation of the dimeric copper(I) precursor $\left[\mathrm{Me}_{2} \mathrm{NC}(\mathrm{NiPr})_{2} \mathrm{Cu}\right]_{2}(\mathbf{1})$ have been investigated with TOF MS, MI FTIR spectroscopy, and DFT calculations. The experimental results showed that precursor 1 fragments above oven temperatures of 150 ${ }^{\circ} \mathrm{C}$ to release the oxidized guanidine $\mathrm{Me}_{2} \mathrm{NC}(=\mathrm{Ni} \mathrm{Pr})\left(\mathrm{N}=\mathrm{CMe}_{2}\right)(\mathbf{3})$. In addition to 3, the formation of $\mathrm{H}_{2}$ was detected by TOF MS. The key intermediate 3 was synthesized independently in this study and through comparison of FTIR spectra of matrix-isolated $\mathbf{3}$ with those from thermolysis experiment with copper precursor $\mathbf{1}$, the formation of the oxidized guanidine $\mathbf{3}$ as a product of the thermolysis was proven. The structure of the major IR bands could be rationalized with the help of DFT calculations, which showed that species 3 exists as a predomination of the trans isomer. These findings can be explained by assuming a $\beta$-hydrogen elimination of the ligands in precursor 1 takes place. DFT calculations indicated that the two decomposition mechanisms result from the same parent species (A1), which rationalizes the fact that carbodiimide deinsertion is seen at lower temperatures in the solution phase, but $\beta$-hydrogen elimination is favoured in higher temperature, gas-phase reactions.

When higher temperatures were explored, it became evident that both species 2 and species 3 underwent deinsertion to form free amine and either CDI or 4, respectively. This important observation indicates that CDI detected at high temperatures can result from deinsertion from a thermolysis product, and not necessarily from the precursor itself. 


\subsection{Experimental}

General Remarks. Complex 1 was synthesized via known procedures ${ }^{11}$ and purified by sublimation prior to use $\left(1: 70-77^{\circ} \mathrm{C}\right.$, approximately $\left.10^{-2}-10^{-3} \mathrm{mbar}\right)$. Compound $\mathbf{2}$ and was synthesized via known procedures ${ }^{22}$ and purified via flask-to-flask condensation at ambient temperatures (approx. $10^{-2}-10^{-3}$ mbar).

The matrix isolation and the time-of-flight MS apparatus were separate instruments each equipped with its own thermolysis oven, both identical in construction. Both instruments used the same carrier gas supply and were in close proximity so that the heatable container, loaded with the compound of interest, could be moved from one to the other instrument without exposing the sample to air. Both instruments used the same thermolysis oven temperature controller. The facility was constructed in this way so that a series of experiments with one sample batch could be performed using one method, e.g. MI FTIR spectroscopy, followed by a series of experiments using the other method, e.g. TOF MS, and still being able to keep the experimental conditions similar in both consecutive series of experiments.

Each thermolysis oven was equipped with an $\mathrm{Al}_{2} \mathrm{O}_{3}$ tube $(\mathrm{OD}=4 \mathrm{~mm})$ with two parallel, inner canals $\left(\mathrm{ID}=1 \mathrm{~mm}\right.$ ). The overall length of the $\mathrm{Al}_{2} \mathrm{O}_{3}$ tube was $35 \mathrm{~mm}$ with the last $10 \mathrm{~mm}$ heated with a tungsten wire, coiled around the outside and coated with $\mathrm{ZrO}_{2}$ cement. One of the inner canals was equipped with a thermocouple (Thermocoax: $\mathrm{NiCrSi} / \mathrm{NiSi}$ ), while the second canal was used to let a carrier gas / sample mixture pass through. With this setup, reliable thermolysis temperatures at the inside of the thermolysis tube could be measured without exposing the thermocouple to the gaseous sample. A schematic drawing of the thermolysis oven can be found in reference 2423. 
A thermolysis series with compound $\mathbf{1}$ was done in the temperature range between 100 and $800^{\circ} \mathrm{C}$ for both MI FTIR spectroscopy and TOF MS. The thermolysis of compound 2 was investigated in the temperatures range 200 to $800{ }^{\circ} \mathrm{C}$ for both MI FTIR spectroscopy and TOF MS.

Matrix-isolation FTIR spectroscopy. The matrix apparatus consisted of a vacuum line (Varian TV 551 Navigator; Varian DS 402) and a Sumitomo CSW-71 cryogenic closed cycle system (Sumitomo Heavy Industries. Ltd.) including a Sumitomo RDK415D 4K cold head fitted with CsI windows (vacuum shroud design and fabrication by Janis Research Company, Inc.). The sample was loaded into a heatable container connected to the thermolysis oven. In a typical experiment, the evacuated container, loaded with the compound of interest, was kept at constant temperature $\left(1: 86^{\circ} \mathrm{C} ; 2: 0\right.$ $\left.{ }^{\circ} \mathrm{C}\right)$ while a flow of argon $(1.25 \mathrm{sccm}$; Praxair 6.0$)$ was conducted over the sample (massflow controller: MKS Type 1179A). The resulting gaseous mixture was passed through a thermolysis oven for 30-90 min and deposited onto a CsI window cooled to $20 \mathrm{~K}$. The distance between the end of the heated oven tube and the CsI window was $54 \mathrm{~mm}$. The so-prepared matrices were cooled to 4K for FTIR measurements (Bruker Tensor 27 FTIR; 200 to $5000 \mathrm{~cm}^{-1}$; resolution of $1 \mathrm{~cm}^{-1}$ ).

Time-of-flight mass spectrometry (TOF MS). The apparatus consisted of a custom designed vacuum chamber (designed by Stefan Kaesdorf, Geräte für Forschung und Industrie; built by Johnsen Ultravac Inc.), equipped with one roughing vacuum pump (Varian DS 602) and three turbo vacuum pumps, and a reflectron time-of-flight spectrometer with electron impact ionization (Stefan Kaesdorf, Geräte für Forschung und Industrie; time resolution under favorable conditions $\mathrm{T} / \Delta \mathrm{T}=10000)$. For more details 
please see reference 12 (Note that the distance between the end of the heated oven tube to the orifice of the skimmer was shortened from 12 to $8 \mathrm{~mm}$.). In a typical experiment, the sample container was kept at constant temperature $\left(1: 90-92{ }^{\circ} \mathrm{C} ; 2: 0{ }^{\circ} \mathrm{C}\right)$ while a flow of argon was conducted over the sample. To get acceptable signal to noise ratios, the container temperature for $\mathbf{1}$ needed for the TOF MS measurements was slightly higher than those for MI FTIR spectroscopy (see above). For all measurements electron impact of $70 \mathrm{eV}$ and 1 to 20 million sweeps per oven temperature were applied. The signal of the carrier gas argon at $40 \mathrm{u}$ was reduced in intensity by using a mass filter at $4.35 \mathrm{kV}$ (delay: $3.1000 \mu \mathrm{s}$; duration: $0.9000 \mu \mathrm{s})$. This prevented the MCP detector from exceeding its detection limit. Calibration of flight times was done within every new series of measurements using Xe as an additional standard. Calibrations were done with the following masses: ${ }^{129} \mathrm{Xe}^{+}(128.90478 \mathrm{u}),{ }^{129} \mathrm{Xe}^{2+}(64.45239 \mathrm{u}),{ }^{40} \mathrm{Ar}^{+}(39.96230 \mathrm{u})$ and $\mathrm{H}_{2}{ }^{16} \mathrm{O}^{+}(18.01056 \mathrm{u})$. As the calibration masses did not span the entire range of measured ions from 1, the experimental masses were usually within the range of $\pm 0.1 \mathrm{u}$ of the calculated ones.

Calculated masses of selected ions (intensities in parenthesis): $\mathbf{1}^{+}\left(\mathrm{C}_{18} \mathrm{H}_{40} \mathrm{Cu}_{2} \mathrm{~N}_{6}{ }^{+}\right)$: $m / z=466.19(100.0 \%), 467.19(19.5 \%), 467.19(2.2 \%), 468.19(89.1 \%), 468.20(1.8$ \%), 469.19 (17.4 \%), 469.19 (2.0 \%), 470.19 (19.9\%), 470.20 (1.6 \%), 471.19 (3.9\%).

DFT Calculations. All calculations were done using the GAUSSIAN 03 program package. ${ }^{24}$ The mechanism of thermolysis of $\mathbf{1}$ was studied with the $\mathrm{B} 3^{27} \mathrm{LYP}^{28}$ functional and the $6-31+G(d, p)$ basis set augmented with dispersion correcting potentials (DCPs). ${ }^{29}$ DCPs are atom-centered effective core potentials which are employed to better account for long-range dynamical correlation effects (i.e. dispersion interactions). 
Coefficients for DCPs have been optimized ${ }^{30}$ for a wide variety of functionals and basis sets and can be implemented into quantum chemical software simply by appending the input file. All thermodynamic values discussed in the text are calculated free energies at $260^{\circ} \mathrm{C}$. To help interpret the IR spectra acquired, molecular vibrations were studied at the BLYP/6-31G(d) level of theory. The BLYP functional was used rather than B3LYP as it is known that unscaled frequencies obtained from BLYP are more accurate than those from B3LYP. ${ }^{31}$ 


\subsection{Supplemental Information}

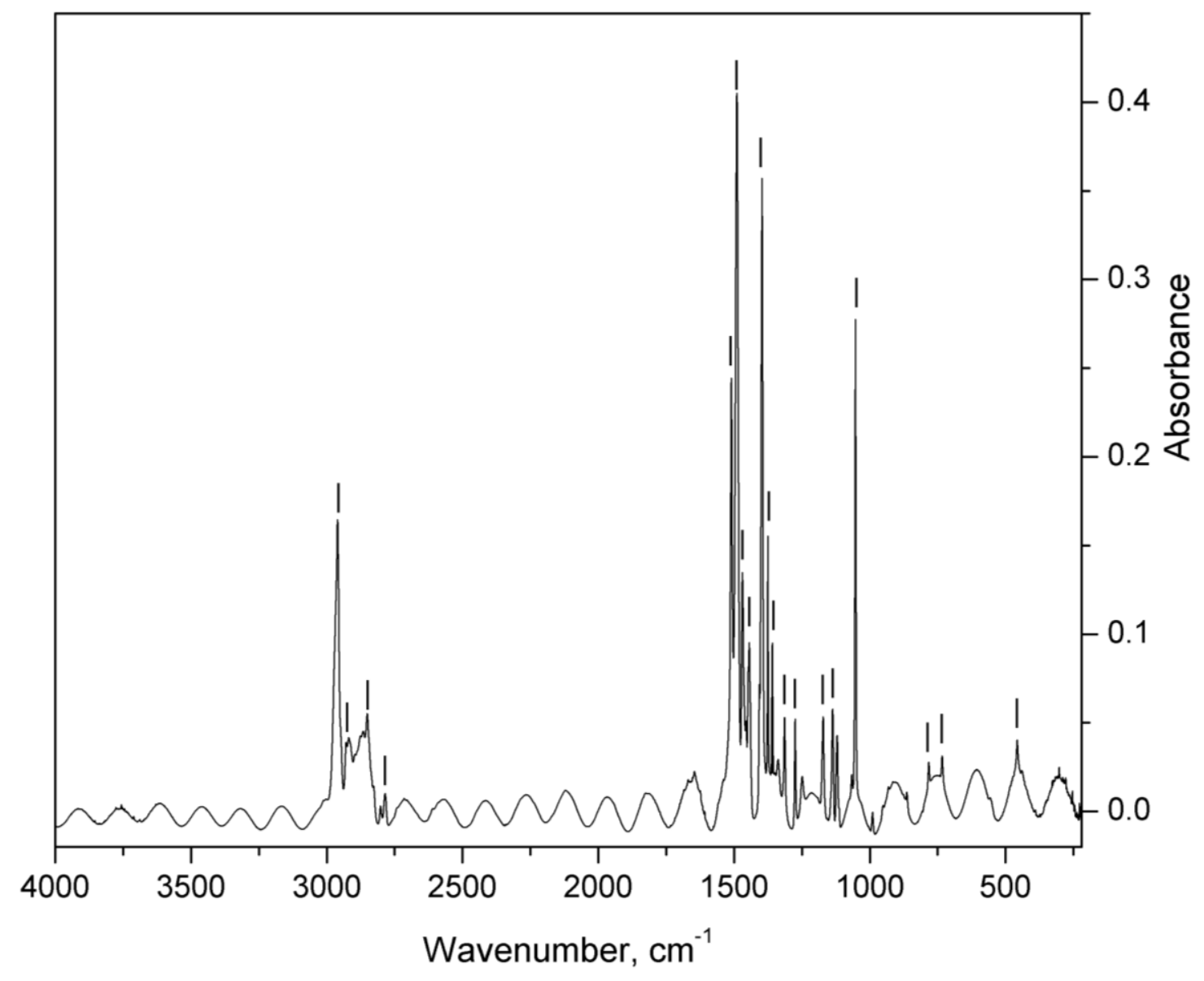

Figure 3.11 Species 1 in solid argon (oven temperature at $140{ }^{\circ} \mathrm{C}$; no decomposition; 220 $-4000 \mathrm{~cm}^{-1}$ ). Wavenumbers $\left[\mathrm{cm}^{-1}\right]$ of the marked IR bands are 2960.4, 2919.2, 2850.9, 2785.3, 1510.1, 1489.7, 1467.0, 1444.0, 1397.0, 1375.7, 1359.2, 1314.2, 1275.3, 1171.9, $1137.2,1053.0,783.2,733.8,457.4$. 


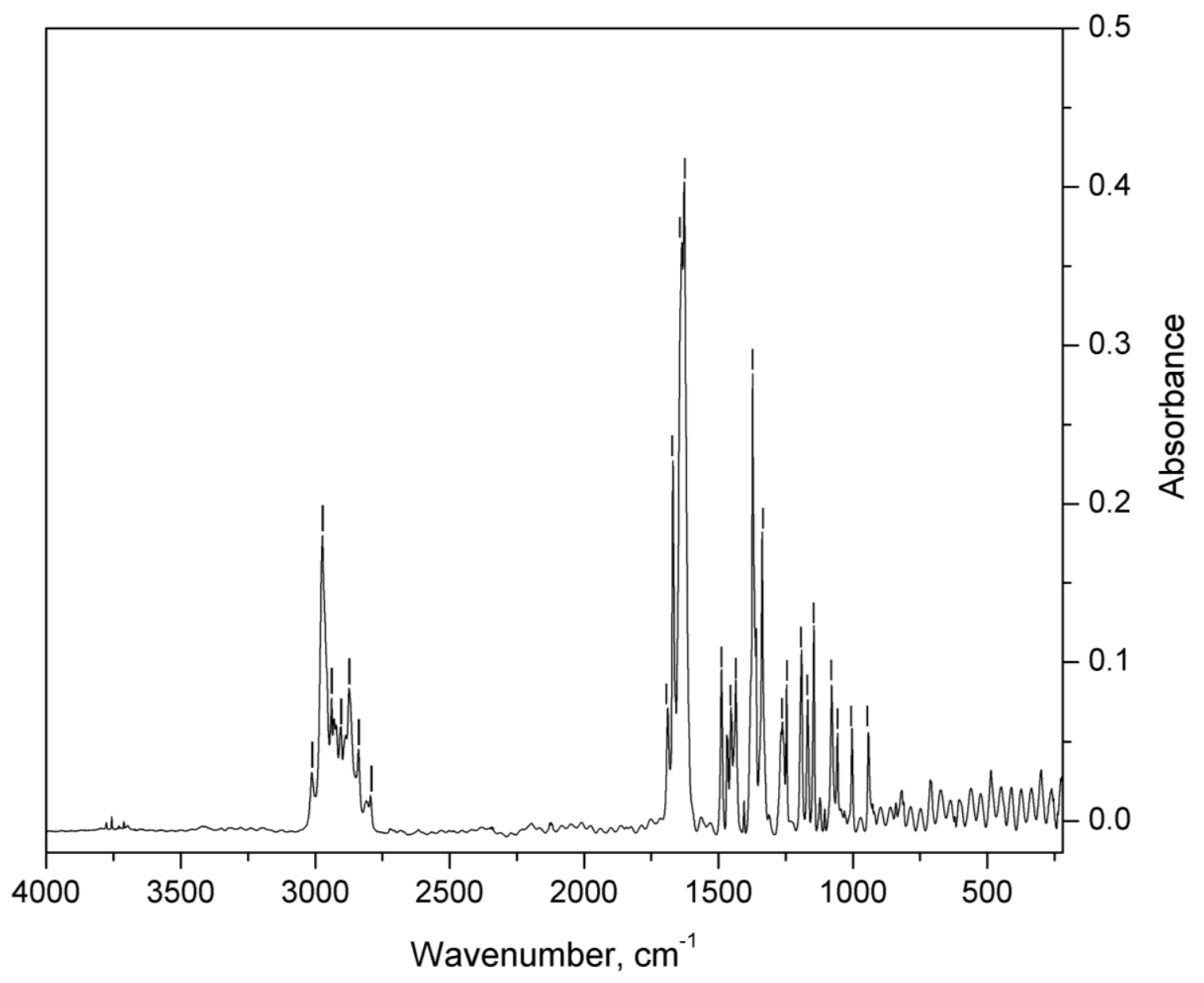

Figure 3.12 Results of a thermolysis of complex 1 at $260{ }^{\circ} \mathrm{C}\left(220-4000 \mathrm{~cm}^{-1}\right.$; same IR spectrum as Figure 3.6B). Wavenumbers $\left[\mathrm{cm}^{-1}\right]$ of the marked IR bands are 3012.8, 2972.8, 2939.2, 2904.6, 2874.2, 2839.4, 2794.6, 1687.9, 1668.9, 1635.1, 1626.6, 1488.4, $1452.9,1435.4,1372.5,1337.4,1261.9,1246.2$, 1190.7, 1167.9, 1144.8, 1079.1, 1056.6, 1003.2, 942.5 . 


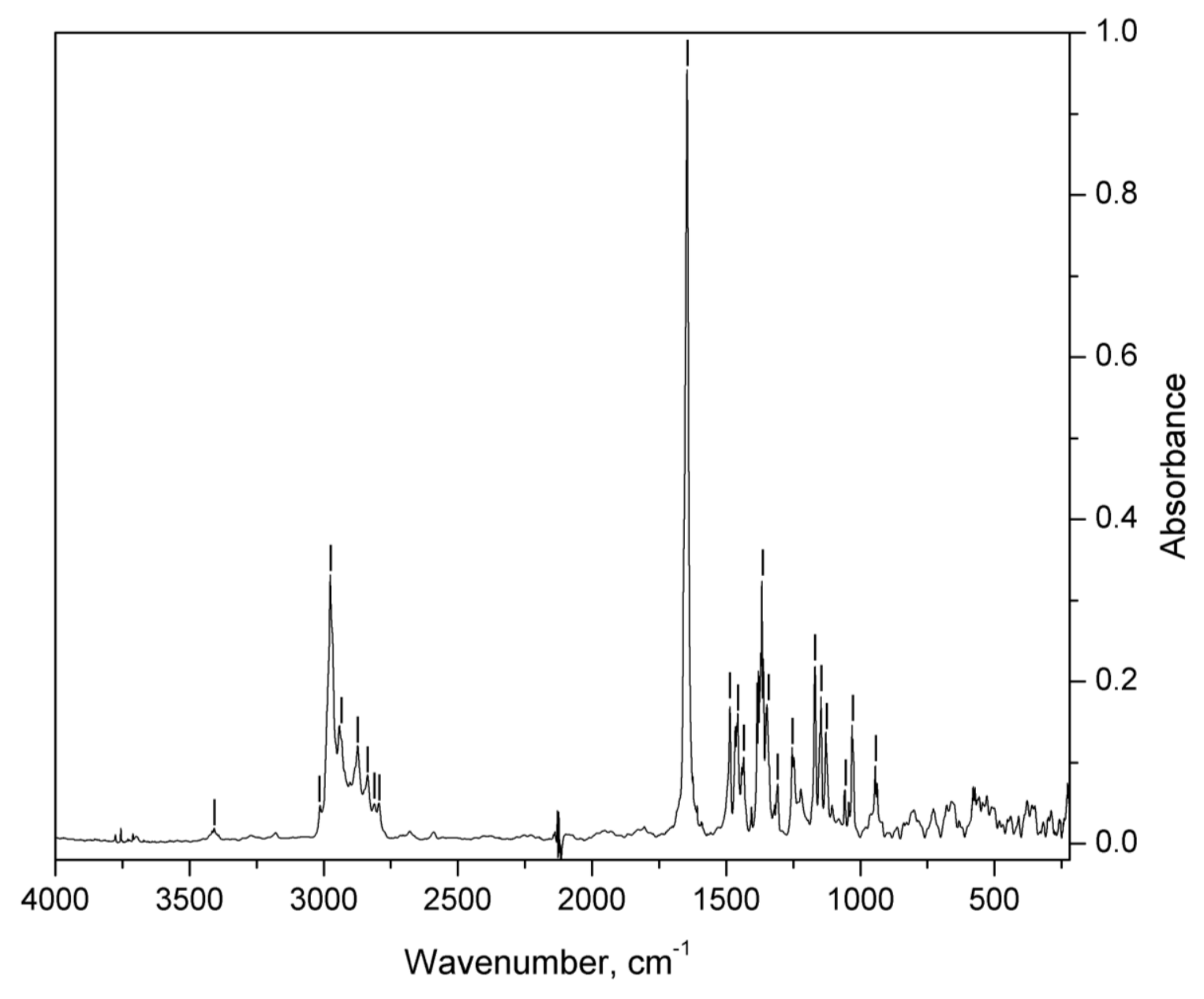

Figure 3.13 Species 2 in solid argon (thermolysis oven at $260{ }^{\circ} \mathrm{C}$; no decomposition; 220 $-4000 \mathrm{~cm}^{-1}$; same IR spectrum as Figure 3.6A). The sample of 2 contained residual amounts of $i \operatorname{PrNCN} i \operatorname{Pr}(\mathrm{CDI})$, which were electronically eliminated from the IR spectrum of 2. Wavenumbers [ $\mathrm{cm}^{-1}$ ] of the marked IR bands are 3408.7, 3013.9, 2975.9, 2941.7, 2873.5, 2836.6, 2811.4, 2794.2, 1646.0, 1486.2, 1457.0, 1435.1, 1367.3, 1348.3, 1308.1, $1254.3,1169.3,1146.8,1129.2,1058.7,1031.4,937.3$. 


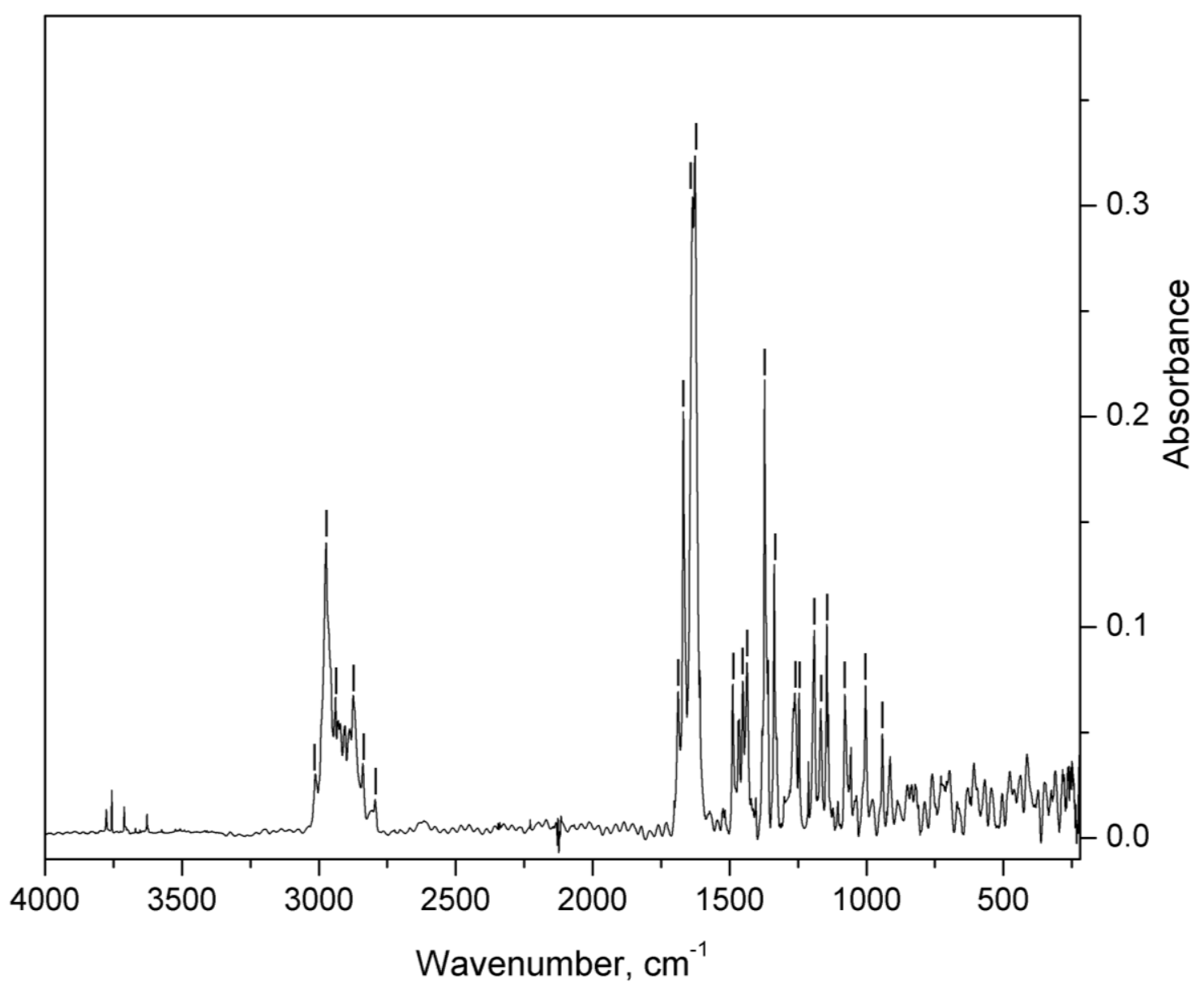

Figure 3.14 Species 3 in solid argon (thermolysis oven at $260{ }^{\circ} \mathrm{C}$; no decomposition; 220 $-4000 \mathrm{~cm}^{-1}$; same IR spectrum as Figure 3.6C). The sample of 2 contained small amounts of $i \operatorname{PrNCN} i \operatorname{Pr}(\mathrm{CDI})$, which were electronically eliminated from the spectrum of 3. Wavenumbers $\left[\mathrm{cm}^{-1}\right]$ of the marked IR bands are 3012.9, 2973.4, 2939.3, 2874.6, $2839.5,2794.5,1687.9,1668.9,1634.9,1626.7,1488.5,1452.2,1436.8,1372.3,1337.3$, $1261.9,1246.1,1190.7,1167.6,1144.6,1079.4,1003.2,942.5$. 


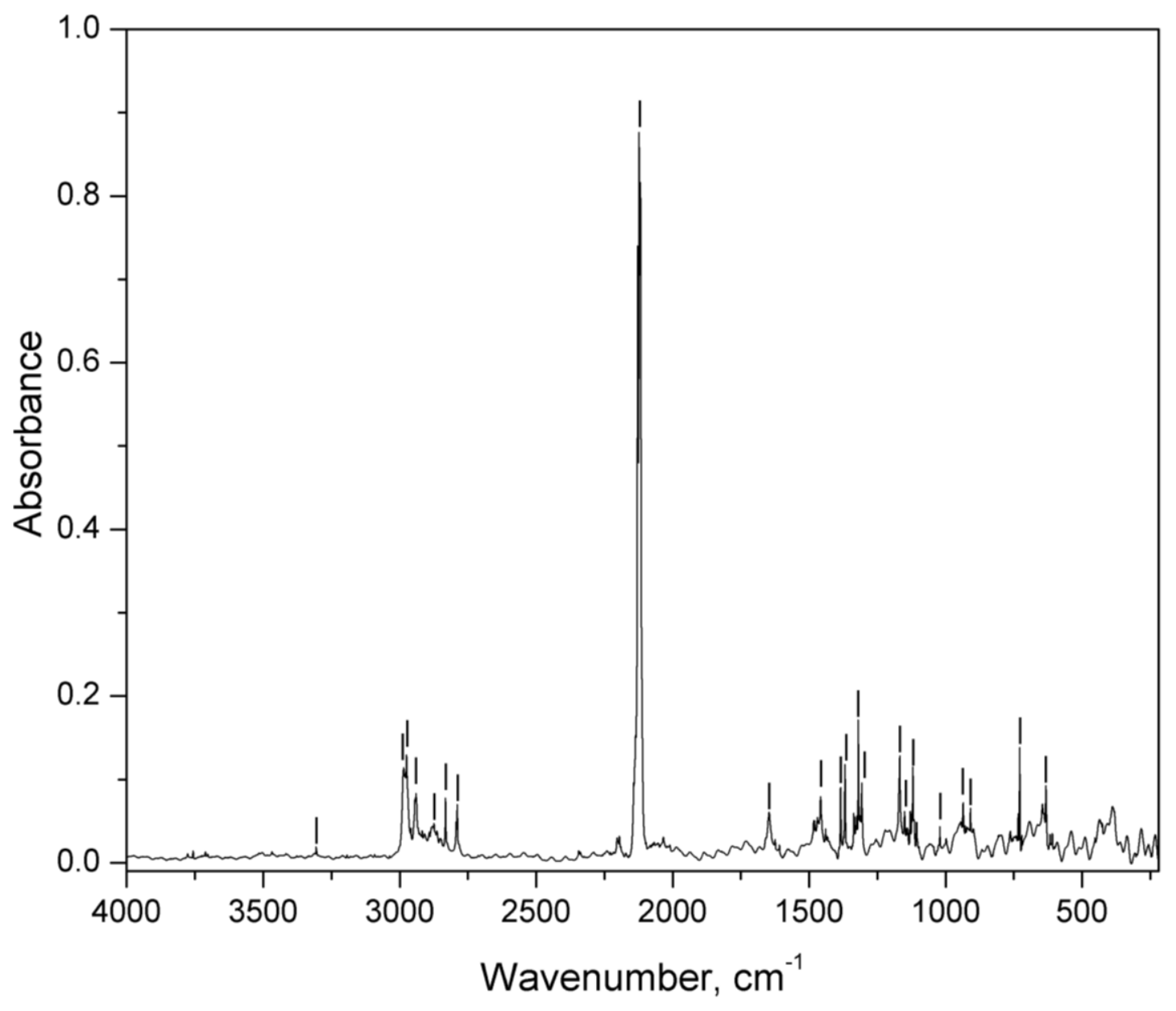

Figure 3.15 Results of a thermolysis of complex 2 at $700{ }^{\circ} \mathrm{C}\left(220-4000 \mathrm{~cm}^{-1}\right)$. Wavenumbers $\left[\mathrm{cm}^{-1}\right]$ of the marked IR bands are (assignments in parentheses) 3306.0, 2985.6 CDI), 2975.0 $\left(\mathrm{HNMe}_{2}\right), 2940.6\left(\mathrm{HNMe}_{2}\right), 2874.4\left(\mathrm{HNMe}_{2}\right), 2832.3\left(\mathrm{HNMe}_{2}\right)$, $2789.5\left(\mathrm{HNMe}_{2}\right), 2123.5$ (CDI; the structure of this IR band changes depending on the way CDI is deposited in matrices; see reference L. C. Ziffle, A. P. Kenney, S. T. Barry and J. Müller, Polyhedron, 2008, 27, 1832-1840.), 1645.9 (most intense peak of 2), 1457.6 ( $\mathrm{HNMe}_{2}$ ), 1384.5 (CDI), 1368.4 (CDI), 1319.6 (CDI), 1306.7 (CDI), 1167.7 (CDI), $1150.4\left(\mathrm{HNMe}_{2}\right), 1119.8$ (CDI), $1020.9\left(\mathrm{HNMe}_{2}\right), 935.8$ (CDI), 908.6, 728.6 (most intense peak of $\mathrm{HNMe}_{2}$ ), 632.6 (CDI). The assignment of IR bands to $\mathrm{HNMe}_{2}$ is based on an independently measured IR spectrum of matrix-isolated $\mathrm{HNMe}_{2}$ in argon (B. Wittig, J. Müller, unpublished results). 


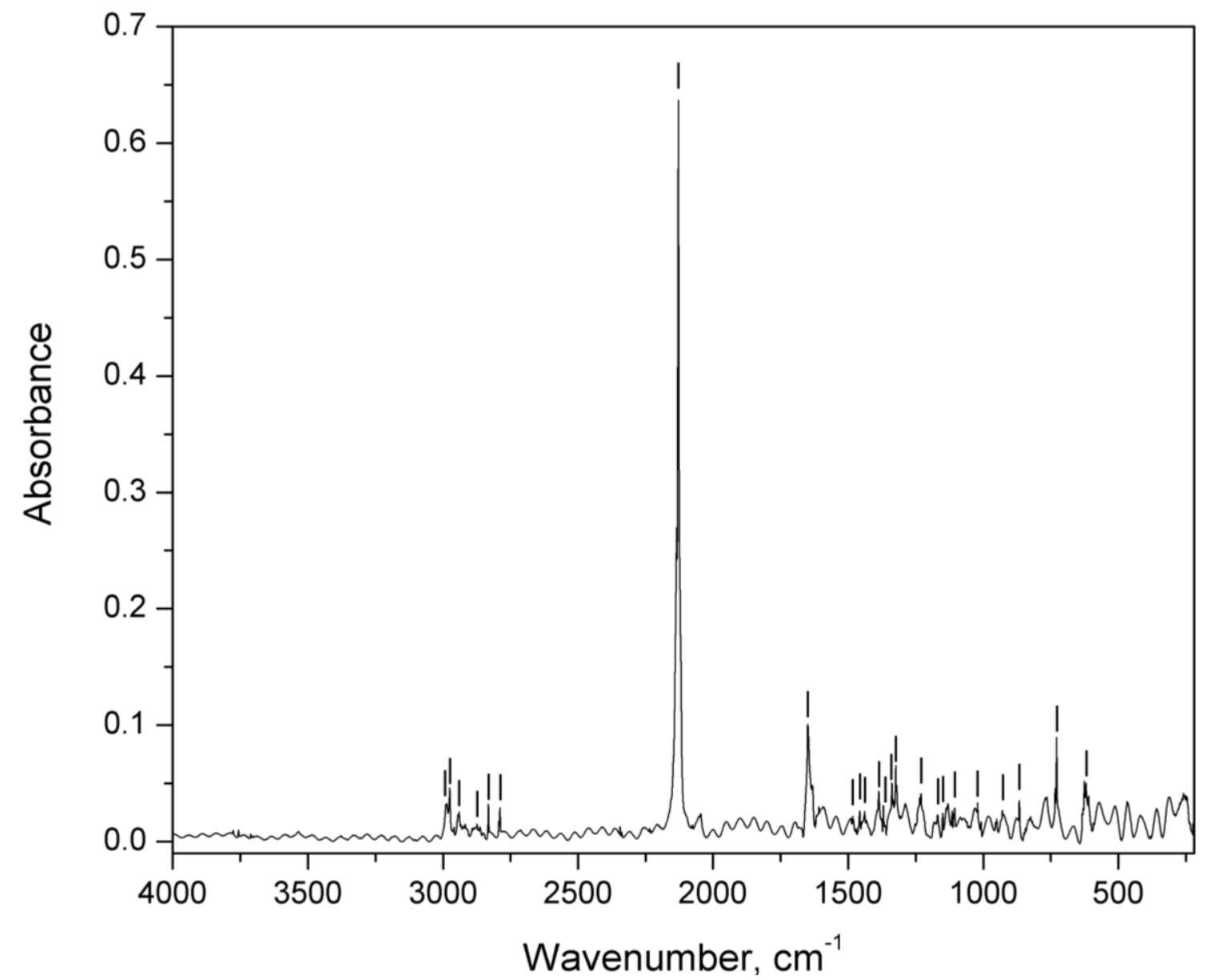

Figure 3.16 Results of a thermolysis of complex 1 at $700{ }^{\circ} \mathrm{C}\left(220-4000 \mathrm{~cm}^{-1}\right)$. Small amounts of $\mathbf{2}$ and $\mathbf{3}$ were electronically eliminated from this spectrum. Wavenumbers $\left[\mathrm{cm}^{-1}\right]$ of the marked IR bands are (assignments in parentheses) 2985.4, $2975.2\left(\mathrm{HNMe}_{2}\right)$, $2940.7\left(\mathrm{HNMe}_{2}\right), 2874.4\left(\mathrm{HNMe}_{2}\right), 2832.3\left(\mathrm{HNMe}_{2}\right), 2789.5\left(\mathrm{HNMe}_{2}\right), 2127.7,1649.2$, $1483.3\left(\mathrm{HNMe}_{2}\right), 1457.3\left(\mathrm{HNMe}_{2}\right), 1439.1\left(\mathrm{HNMe}_{2}\right), 1386.9,1369.6,1337.9,1323.8$, 1230.4, 1169.0, $1150.3\left(\mathrm{HNMe}_{2}\right)$, 1106.0, $1021.0\left(\mathrm{HNMe}_{2}\right), 929.1\left(\mathrm{HNMe}_{2}\right)$, 866.6, 728.7 (most intense peak of $\mathrm{HNMe}_{2}$ ), 626.1. Note: The marked, but unassigned IR bands could be tentatively assigned to the new carbodiimide 4. However, an unambiguous characterization of $\mathbf{4}$ on the basis of the IR data alone is impossible. The IR band at $1649.2 \mathrm{~cm}^{-1}$ could be caused by the $\mathrm{C}=\mathrm{C}$ stretching mode of 4 (see Figure 3.10). The assignment of IR bands to $\mathrm{HNMe}_{2}$ is based on an independently measured IR spectrum of matrix-isolated $\mathrm{HNMe}_{2}$ in argon (B. Wittig, J. Müller, unpublished results). 


\subsection{References}

1 Chen, T. Y.; Omnes, L.; Vaisserman, J.; Doppelt, P. Inorg. Chim. Acta 2004, 357, 1299-1302.

2 Bollmann, D.; Merkel, R.; Klumpp, A. Microelectron. Eng. 1997, 37-8, 105-110.

3 Lagalante, A. F.; Hansen, B. N.; Bruno, T. J.; Sievers, R. E. Inorg. Chem. 1995, 34, 5781-5785.

4 Wenzel, T. J.; Williams, E. J.; Haltiwanger, R. C.; Sievers, R. E. Polyhedron 1985, 4, 369-378.

5 Park, K. H.; Marshall, W. J. J. Am. Chem. Soc. 2005, 127, 9330-9331.

6 Li, Z. W.; Rahtu, A.; Gordon, R. G. J. Electrochem. Soc. 2006, 153, C787-C794.

7 Li, Z. W.; Barry, S. T.; Gordon, R. G. Inorg. Chem. 2005, 44, 1728-1735.

8 Lim, B. S.; Rahtu, A.; Gordon, R. G. Nature Materials 2003, 2, 749-754.

9 Lim, B. S.; Rahtu, A.; Park, J. S.; Gordon, R. G. Inorg. Chem. 2003, 42, 7951-7958.

10 Coyle, J. P.; Monillas, W. H.; Yap, G. P. A.; Barry, S. T. Inorg. Chem. 2008, 47, 683-689.

11 Wu, J. P.; Li, J. Y.; Zhou, C. G.; Lei, X. J.; Gaffney, T.; Norman, J. A. T.; Li, Z. W.; Gordon, R.; Cheng, H. S. Organometallics 2007, 26, 2803-2805.

12 Ziffle, L. C.; Kenney, A. P.; Barry, S. T.; Müller, J. Polyhedron 2008, 27, $1832-$ 1840 .

13 Whittle, E.; Dows, D. A.; Pimentel, G. C. J. Chem. Phys. 1954, 22, 1943.

14 Norman, I.; Pimentel, G. C. Nature 1954, 174, 508-509.

15 Only traces of $\mathbf{1}^{+}$were detectable; intensity of $466.3 \mathrm{u}$ was 25 counts out of 10 million sweeps.

16 This value was calculated using $\Delta \mathrm{G}=-127.3 \mathrm{kcal} / \mathrm{mol}$ for the process $2 \mathrm{Cu}_{(\mathrm{g})} \rightarrow 2$ $\mathrm{Cu}_{(\mathrm{s})}$ calculated from data tabulated in: Lide, D. R. Handbook of Chemistry and Physics $79^{\text {th }}$ Edition; CRC Press: Boca Raton, 1998. Using the same level of theory, $\Delta \mathrm{G}$ is computed to be $-44.4 \mathrm{kcal} / \mathrm{mol}$ for the CDI deinsertion process shown in Figure 3.1. 
17 Dai, M.; Kwon, J.; Halls, M. D.; Gordon, R. G.; Chabal, Y. J. Langmuir 2010, 26,3911-3917

18 Ma, Q.; Guo, H.; Gordon, R. G.; Zaera, F. Chem. Mater., 2010, 22, 352-359.

19 Whitesides, G. M.; Stedronsky, E. R.; Casey, C. P.; San Filippo, J. J. Am. Chem. Soc. 2002, 92, 1426-1427.

20 Whitesides, G. M.; Casey, C. P.; Krieger, J. K. J. Am. Chem. Soc. 2002, 93, 13791389.

21 Whitesides, G. M.; Panek, E. J.; Stedronsky, E. R. J. Am. Chem. Soc. 2002, 94, 232239.

22 Tsuda, T.; Watanabe, K.; Miyata, K.; Yamamoto, H.; Saegusa, T. Inorg. Chem. 1981, 20, 2728-2730.

23 Yamaguchi, J.; Takeda, T. Chem. Lett. 1992, 1933-1936.

24 Frisch, M. J.; Trucks, G. W.; Schlegel, H. B.; Scuseria, G. E.; Robb, M. A.; Cheeseman, J. R.; J. A. Montgomery, J.; Vreven, T.; Kudin, K. N.; Burant, J. C.; Millam, J. M.; Iyengar, S. S.; Tomasi, J.; Barone, V.; Mennucci, B.; Cossi, M.; Scalmani, G.; Rega, N.; Petersson, G. A.; Nakatsuji, H.; Hada, M.; Ehara, M.; Toyota, K.; Fukuda, R.; Hasegawa, J.; Ishida, M.; Nakajima, T.; Honda, Y.; Kitao, O.; Nakai, H.; Klene, M.; Li, X.; Knox, J. E.; Hratchian, H. P.; Cross, J. B.; Bakken, V.; Adamo, C.; Jaramillo, J.; Gomperts, R.; Stratmann, R. E.; Yazyev, O.; Austin, A. J.; Cammi, R.; Pomelli, C.; Ochterski, J. W.; Ayala, P. Y.; Morokuma, K.; Voth, G. A.; Salvador, P.; Dannenberg, J. J.; Zakrzewski, V. G.; Dapprich, S.; Daniels, A. D.; Strain, M. C.; Farkas, O.; Malick, D. K.; Rabuck, A. D.; Raghavachari, K.; Foresman, J. B.; Ortiz, J. V.; Cui, Q.; Baboul, A. G.; Clifford, S.; Cioslowski, J.; Stefanov, B. B.; Liu, G.; Liashenko, A.; Piskorz, P.; Komaromi, I.; Martin, R. L.; Fox, D. J.; Keith, T.; Al-Laham, M. A.; Peng, C. Y.; Nanayakkara, A.; Challacombe, M.; Gill, P. M. W.; Johnson, B.; Chen, W.; Wong, M. W.; Gonzalez, C.; Pople, J. A. Gaussian 03; Revision D.02 ed.; Gaussian, Inc.: Wallingford CT, 2004.

25 Farrugia, L. J. J. Appl. Crystallogr. 1997, 30, 565.

26 Müller, J.; Wittig, B.; Sternkicker, H.; Bendix, S. J. Phys. IV 2001, 11, 17-22.

27 Becke, A. D. J. Chem. Phys. 1993, 98, 5648-5652.

28 Lee, C.; Yang, W.; Parr, R. G. Phys. Rev. B 1988, 37, 785-789.

29 DiLabio, G. A. Chem. Phys. Lett. 2008, 455, 348-353. 
30 Mackie, I. D.; DiLabio, G. A. J. Phys. Chem. A 2008, 112, 10968-10976.

31 Rauhut, G.; Pulay, P. J. Phys. Chem. 1995, 99, 3093-3100. 


\section{Chapter: Copper Iminopyrrolidinates}

Modified from original manuscripts published as:

Coyle, J. P. ${ }^{1}$; Kurek, A. ${ }^{1}$; Pallister, P. J. ${ }^{1}$; Sirianni, E. R. ${ }^{2}$; Yap, G. P. A. ${ }^{2}$; Barry, S. T. ${ }^{1}$ "Preventing thermolysis: precursor design for volatile copper compounds" Chem. Commun. 2012, 48, 10440-10442.

Coyle, J. P. ${ }^{1}$; Pallister, P. J. ; Kurek, A. ${ }^{1}$; Sirianni, E. R. ${ }^{2}$; Yap, G. P. A. ${ }^{2}$; Barry, S. T. ${ }^{1}$ "Copper Iminopyrrolidinates: A Study of Thermal and Surface Chemistry" Inorg. Chem. 2013, 52, 910-917

${ }^{1}$ Department of Chemistry, Carleton University, 1125 Colonel By Drive, Ottawa, Ontario, Canada

${ }^{2}$ Department of Chemistry \& Biochemistry, University of Delaware, Newark, United States

\subsection{Abstract}

Several copper(I) iminopyrrolidinates have been evaluated by thermogravimetric analysis (TGA) and solution based ${ }^{1} \mathrm{H}$ NMR studies to determine their thermal stability and decomposition mechanisms. Iminopyrrolidinates were used as a ligand for copper(I) to block previously identified decomposition routes of carbodiimide deinsertion and $\beta$ hydrogen abstraction. The compounds copper(I) isopropyl-iminopyrrolidinate, (1), copper(I) tertbutyl-iminopyrrolidinate, (2) and copper (I) tert-butyl-imino-2,2dimethylpyrrolidinate, (3) were synthesized for this study, and compared to the previously reported copper(I) guanidinate $\left[\mathrm{Me}_{2} \mathrm{NC}\left({ }^{\mathrm{i}} \mathrm{PrN}\right)_{2} \mathrm{Cu}\right]_{2}(\mathbf{4})$. Compounds $\mathbf{1}$ and $\mathbf{2}$ were found to be volatile yet susceptible to decomposition during TGA. At $165^{\circ} \mathrm{C}$ in $\mathrm{C}_{6} \mathrm{D}_{6}$, they had half-lives of $181.7 \mathrm{~h}$ and $23.7 \mathrm{~h}$, respectively. The main thermolysis product of $\mathbf{1}$ and $\mathbf{2}$ was their respective protonated iminopyrrolidine ligand. $\beta$-hydrogen abstraction was proposed for the mechanism of thermal decomposition. Copper(I) tertbutyl-imino-2,2-dimethylpyrrolidinate (3) demonstrated superior thermal stability and 
showed negligible decomposition in TGA experiments up to $300{ }^{\circ} \mathrm{C}$ as well as no decomposition in solutions at $165^{\circ} \mathrm{C}$ over 3 weeks. Since compound $\mathbf{3}$ showed no thermolysis at $165^{\circ} \mathrm{C}$, it was further studied by chemisorption on high surface area silica. It was found to eliminate an isobutene upon chemisoption at $275^{\circ} \mathrm{C}$.

\subsection{Introduction}

Copper metal remains an interesting topic for chemical vapour deposition (CVD) and atomic layer deposition (ALD) due to its use in microelectronics, primarily as an interconnect. ${ }^{1}$ One class of particularly well-studied precursors are the amidinates, which originated from the Gordon group in the early 2000s. ${ }^{2}$ Copper amidinates have utility in $\mathrm{ALD}^{2 \mathrm{a}}$ and $\mathrm{CVD}^{3}$ processes between temperatures of $150-240^{\circ} \mathrm{C}$ when using hydrogen as a reducing agent. Copper(I)-N,N'-di-isopropylacetamidinate has been shown to undergo CVD deposition as low as $140^{\circ} \mathrm{C},{ }^{4}$ and produces free amidine, acetonitrile, propene and iminopropane when allowed to thermally decompose in the absence of a reducing agent. ${ }^{5}$ These findings have been corroborated in the surface study work of copper(I) acetamidinates on nickel where - above $130^{\circ} \mathrm{C}$ - the self-limiting nature of the monolayer is compromised and continuous uptake of the copper precursor is observed. Similar ligand fragments were identified in the surface work as in the gas phase work.

We have previously investigated the thermal decomposition of copper(I) guanidinates, which are similar to amidinates except that the exocyclic group is an amide, rather than an alkyl. ${ }^{6,7}$ Using a guanidinate with isopropyl groups on the chelating nitrogens, we found two distinct thermal decomposition mechanisms (Figure 4.1). In solution at lower temperatures, the guanidinate deinserted carbodiimide (CDI) and produced the parent amine of the exocyclic amide group. ${ }^{6}$ In the gas phase at greater 
than $150^{\circ} \mathrm{C}$, the methylene carbon of the isopropyl group on the chelating nitrogen loses a hydrogen and produces an oxidized guanidine. Hydrogen gas is also found as a thermolysis side product. ${ }^{7}$

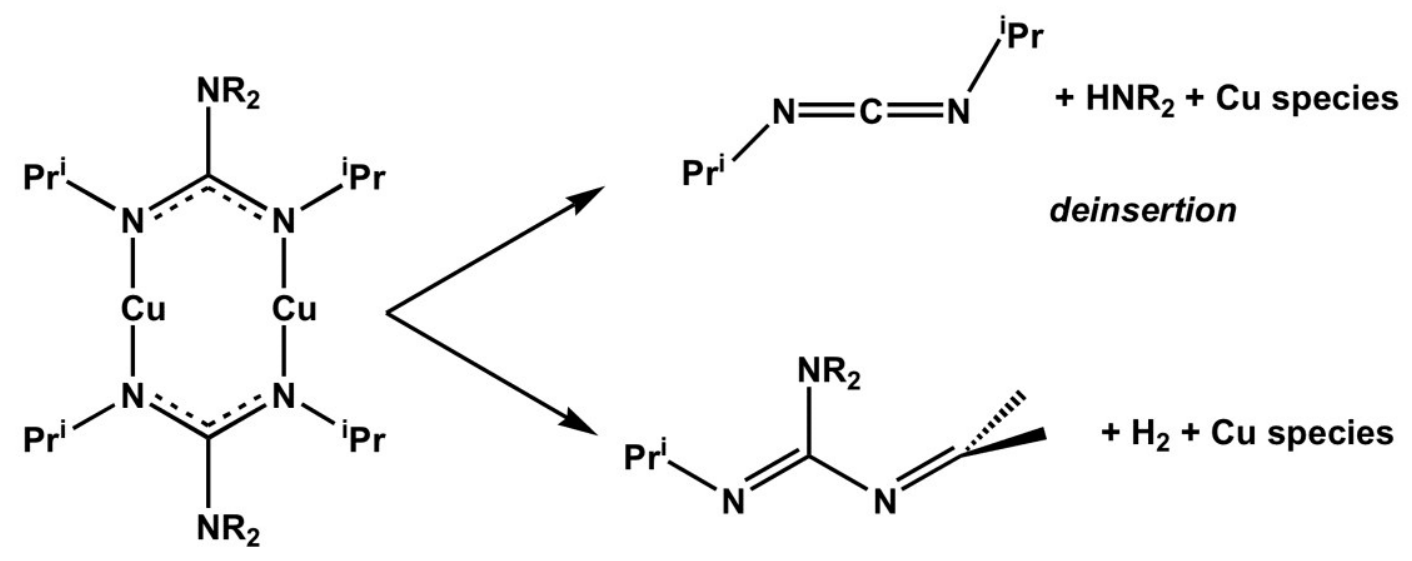

$\beta$-hydrogen elimination

Figure 4.1 Two Thermal Decomposition Pathways For a Copper(I) Guanidiante Species.

Both of these thermal decomposition mechanisms are troubling from the point of view of developing a copper precursor for ALD. In the first case, the elimination of the oxidized guanidinate by $\beta$-hydrogen abstraction produces a copper hydride, which readily eliminates dihydrogen or parent guanidine to produce copper metal. This circumscribes an ALD process in favour of a CVD process. CDI deinsertion also greatly limits the use of this precursor in thermal processes. The formation of a (transient) copper-amide bond creates a species that will readily decompose, ${ }^{8}$ thus also preventing ALD in favour of a CVD process.

A re-design of the basic amidinate framework is necessary to remove the known thermal decomposition pathways of this ligand family. Our group has developed and extensively characterized a novel iminopyrrolidinate ligand family that has evidenced good thermal stability. Recently, we have reported a family of volatile, stable aluminum 
compounds. ${ }^{9}$ This ligand has a five-membered ring linking one chelate nitrogen to the "bridgehead" (i.e., quaternary) carbon of the amidinate, thus preventing CDI deinsertion. Likewise, the ligand has been synthesized to allow control over the number of $\beta$ hydrogens, resulting in a series of three related copper(I) iminopyrrolidinates (Figure 4.2).
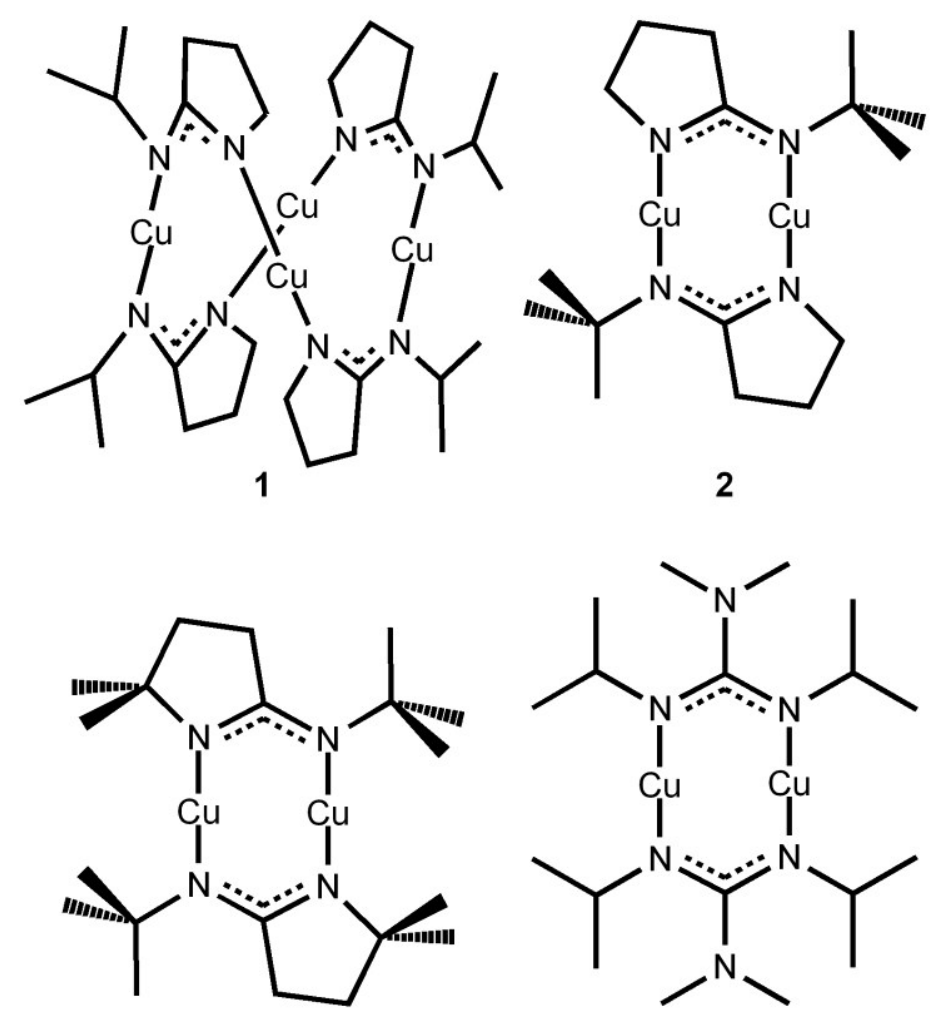

3

4

Figure 4.2 Series of Amidinate-Type Ligands with Differing Numbers of $\beta$-Hydrogens, Shown As Their Corresponding Copper(I) Compounds

Herein we introduce three novel copper iminopyrrolidinates (copper(I) isopropyliminopyrrolidinate, $\mathbf{1}$, copper(I) tertbutyl-iminopyrrolidinate, $\mathbf{2}$ and copper (I) tert-butylimino-2,2-dimethylpyrrolidinate, 3) to complement our previously reported copper (I) $\mathrm{N}^{\prime}, \mathrm{N}$ "-diisopropyl-N,N-dimethyl guanidinate (4). ${ }^{6}$ A thorough exploration of the thermal chemistry of these four compounds was made to demonstrate how the redesign of this ligand has influenced thermal behaviour. We have investigated the thermal stability of 
this series in the solid phase using a variety of thermal gravimetric methods, as well as in solution phase using high resolution ${ }^{1} \mathrm{H}$ NMR of sealed tubes that have been treated at elevated temperatures to study decomposition kinetics. Finally, due to the superior thermal stability of $\mathbf{3}$, we have undertaken a study of the nature of the chemisorbed species produced on silica by 3 as deposited at $275^{\circ} \mathrm{C}$.

\subsection{Results and Discussion}

The copper compounds $\mathbf{1}-\mathbf{3}$ were simply made by salt metathesis of copper(I) chloride with the in-situ generated lithium iminopyrrolidinates. These compounds were isolated as white crystalline materials in moderate to high yields. Similarly, a recent paper describes trimeric, heteroleptic copper(I) guanidinates isolated from the attempted synthesis of $\left[\mathrm{Me}_{2} \mathrm{NC}\left(\mathrm{N}^{\mathrm{t}} \mathrm{Bu}\right)_{2} \mathrm{Cu}\right]_{2} \cdot{ }^{10}$ We believe the ring of the iminopyrrolidinates reported herein was necessary to slightly reduce the steric bulk and permit the isolation of $\mathbf{2}$ and $\mathbf{3}$ as a dimeric species (see below).

The synthesis of several iminopyrrolidines was described previously and used 2pyrrolidone as a starting material. For the synthesis of tert-butyl-imino-2,2dimethylpyrrolidine, a different synthetic route was carried out to increase the conversion yield of 5,5-dimethyl-2-pyrrolidone (Figure 4.3). The decorated 2-pyrrolidone was not commercially available and the work-up for its synthesis was labour intensive. This alternative synthetic route is advantageous as it achieves higher yields at lower temperatures for the substitution of pyrrolidones with bulky amines. 

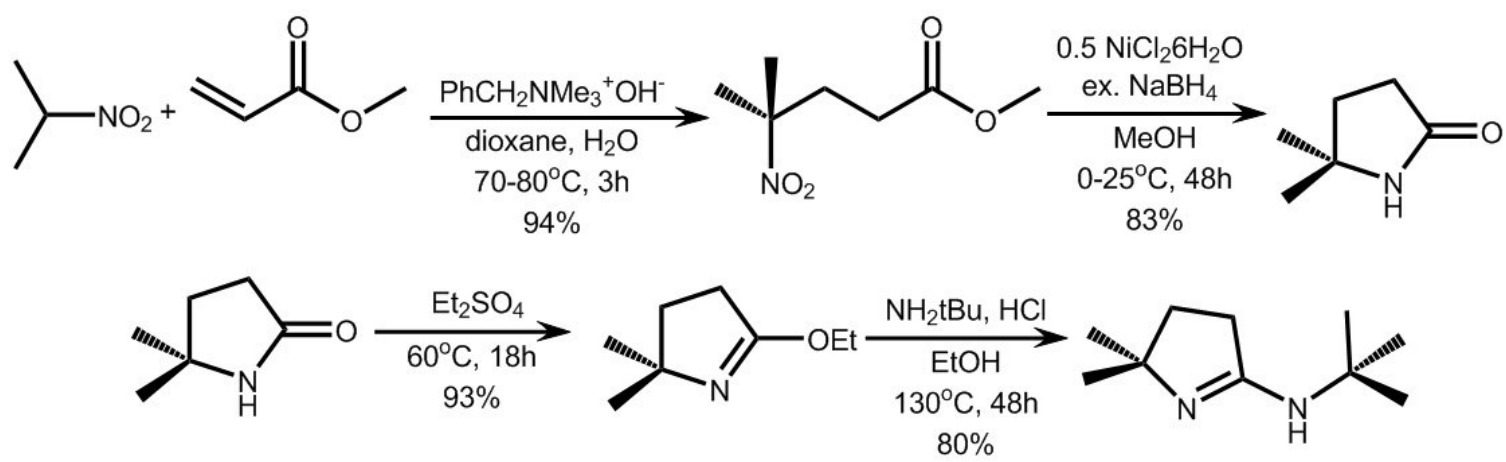

Figure 4.3 The synthetic procedure for producing the tert-butyl-imino-2dimethylpyrrolidine ligand.

The ${ }^{1} \mathrm{H}$ NMR of $\mathbf{2}$ and $\mathbf{3}$ was simple, suggesting a symmetrical oligomeric solution structure, similar to known copper guanidinate dimers. ${ }^{6}$ However, compound $\mathbf{1}$ showed a more complex ${ }^{1} \mathrm{H}$ NMR. The doublets for the methyls of the isopropyl moiety in 1 suggested several environments. It is unclear if these multiple environments were due to an equilibrium of oligomers or from asymmetry in the molecule, but the relative integrations of these doublets were dependant on the concentration of $\mathbf{1}$. Serial dilution of an NMR sample nor heating of the sample to $60^{\circ} \mathrm{C}$ did not yield a trivial set of peaks similar to the spectra of $\mathbf{2}$. Fortunately, $\mathbf{1}$ could be isolated as X-ray quality crystals.

The structure of 1 shows a tetrameric arrangement in the solid state with an orthorhombic Ccca space group (Figure 4.4, Table 4.1). There are 16 tetramers in the unit cell, but only two that are crystallographically distinct. These molecules each have a core of four copper atoms that lay in a rhombohedral plane, with the rhombus of one molecule centred perpendicular to a $\mathrm{C} 2$ axis (with a minor torsion angle of $1.31^{\circ}$ ), and the other parallel to a different $\mathrm{C} 2$ axis (with a torsion angle of $0^{\circ}$ ). The closest $\mathrm{Cu}-\mathrm{Cu}$ contact in the unit cell is $2.76 \AA$, which suggests there is no significant $\mathrm{Cu}-\mathrm{Cu}$ interactions. ${ }^{11}$ This is supported by the fact that the N-Cu-N bonds only deviate from linearity by about $10^{\circ}$, which is likely caused by steric hinderance in the ligands (see 
below). Since the connectivity of the molecules in the unit cell is very similar, only one molecule will be discussed in depth.

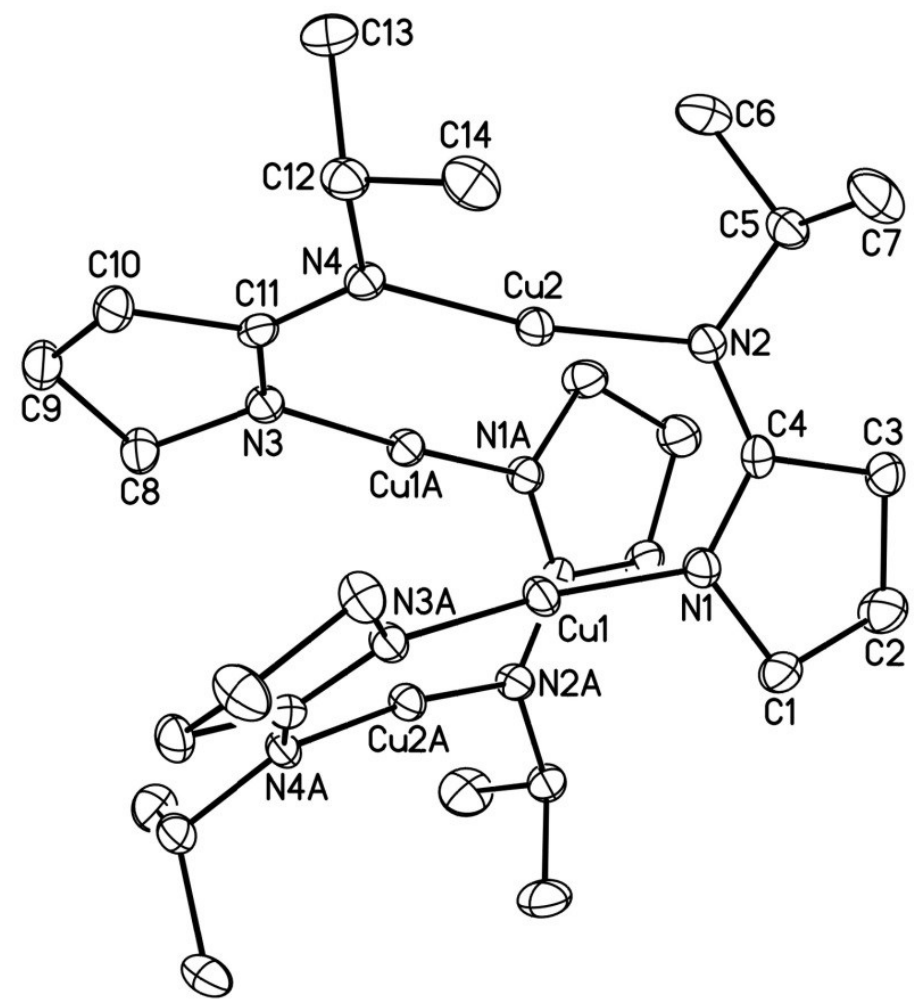

Figure 4.4 Structure of 1, shown with the hydrogens removed for clarity. The thermal ellipsoids are at $30 \%$. 
Table 4.1 Selected bond lengths and angles for $\mathbf{1}$ - $\mathbf{3}$.

\begin{tabular}{|c|c|c|c|}
\hline \multicolumn{2}{|c|}{ Compound 1} & \multicolumn{2}{|c|}{ Compound 2} \\
\hline Selected Bond & Length $(\AA)$ & Selected Bond & Length $(\AA)$ \\
\hline $\mathrm{Cu} 1-\mathrm{Cu} 2$ & $2.756(1)$ & $\mathrm{Cu}-\mathrm{Cu}$ & $2.481(2)$ \\
\hline $\mathrm{Cu} 1-\mathrm{Cu} 1 \mathrm{a}$ & 2.995 & $\mathrm{Cu}-\mathrm{N} 1$ & $1.862(2)$ \\
\hline $\mathrm{Cu} 1-\mathrm{N} 1$ & $1.871(5)$ & $\mathrm{Cu}-\mathrm{N} 2$ & $1.887(2)$ \\
\hline $\mathrm{Cu} 1-\mathrm{N} 3$ & $1.861(5)$ & $\mathrm{N} 1-\mathrm{C} 4$ & $1.336(2)$ \\
\hline $\mathrm{Cu} 2-\mathrm{N} 2$ & $1.885(5)$ & $\mathrm{N} 2-\mathrm{C} 4$ & $1.328(2)$ \\
\hline $\mathrm{Cu} 2-\mathrm{N} 4$ & $1.894(5)$ & Selected Angle & Angle $\left(^{\circ}\right)$ \\
\hline $\mathrm{N} 1-\mathrm{C} 4$ & $1.311(8)$ & $\mathrm{N} 1-\mathrm{Cu}-\mathrm{N} 2$ & $175.29(6)$ \\
\hline $\mathrm{N} 2-\mathrm{C} 4$ & $1.337(8)$ & $\mathrm{N} 1-\mathrm{C} 4-\mathrm{N} 2$ & $122.05(1)$ \\
\hline N3-C11 & $1.311(8)$ & Compound $\mathbf{3}$ & \\
\hline N4-C11 & $1.331(8)$ & Selected Bond & Length $(\AA)$ \\
\hline $\mathrm{N} 1-\mathrm{C} 1$ & $1.443(9)$ & $\mathrm{Cu}-\mathrm{Cu}$ & $2.4726(5)$ \\
\hline $\mathrm{N} 2-\mathrm{C} 5$ & $1.458(9)$ & $\mathrm{Cu}-\mathrm{N} 1$ & $1.867(1)$ \\
\hline $\mathrm{N} 3-\mathrm{C} 8$ & $1.462(8)$ & $\mathrm{Cu}-\mathrm{N} 2$ & $1.887(1)$ \\
\hline $\mathrm{N} 4-\mathrm{C} 12$ & $1.487(9)$ & $\mathrm{N} 1-\mathrm{C} 4$ & $1.330(2)$ \\
\hline Selected Angle & Angle $\left(^{\circ}\right)$ & $\mathrm{N} 2-\mathrm{C} 4$ & $1.328(2)$ \\
\hline $\mathrm{Cu} 2-\mathrm{Cu} 1-\mathrm{Cu} 2$ & $114.13(2)$ & Selected Bond & Length $(\AA)$ \\
\hline $\mathrm{Cu} 1-\mathrm{Cu} 2-\mathrm{Cu} 1$ & $65.86(1)$ & $\mathrm{N} 1-\mathrm{Cu}-\mathrm{N} 2$ & $175.59(6)$ \\
\hline $\mathrm{N} 3-\mathrm{Cu} 1-\mathrm{N} 1$ & $170.5(1)$ & $\mathrm{N} 1-\mathrm{C} 4-\mathrm{N} 2$ & $122.5(1)$ \\
\hline $\mathrm{N} 2-\mathrm{Cu} 2-\mathrm{N} 4$ & $170.5(1)$ & & \\
\hline $\mathrm{N} 1-\mathrm{C} 4-\mathrm{N} 2$ & $123.6(3)$ & & \\
\hline $\mathrm{N} 3-\mathrm{C} 11-\mathrm{N} 4$ & $123.1(3)$ & & \\
\hline
\end{tabular}

Interestingly, the two ligands bonded to any specific copper are bonded through the same type of nitrogen. For example $\mathrm{Cu} 1$ is bonded to the ring nitrogens $\mathrm{N} 1$ and N3, where one might expect the ligands to alternate. Additionally, the isopropyl groups of the ligand are all oriented towards the copper plane. These observations suggest that there is a fine balance of steric interference across the copper plane allowing a tetramer to form. 
This structure type has been seen previously for group 11.12 The $\mathrm{Cu}-\mathrm{N}$ bonds in this molecule have an average length of $1.88 \AA$, which is the same as in $\mathbf{2}$ and $\mathbf{3}$.

Considering the ligands oriented parallel to each other and on the same side of the copper plane, it is obvious that more crowding exists here. The ligands bend out slightly from one another, on average $26.5^{\circ}$ from parallel. As well, there is a slight twist of about $6^{\circ}$ across each ligand with respect to the line defined by the coppers which bind them.

The metallocycle $\mathrm{C}-\mathrm{N}$ bonds are all about equivalent in this molecule $(1.31 \AA$ $1.34 \AA$ ), suggesting complete delocalization of the double bond. The ligand's bridging N$\mathrm{C}-\mathrm{N}$ angle is about $123^{\circ}$, which is slightly larger than $\mathbf{2}-4^{6}{ }^{6}$ This is quite understandable in the case of the tetramer; however, since the ligand is bridging a significantly large $\mathrm{Cu}$ $\mathrm{Cu}$ distance.

The structures of $\mathbf{2}$ and $\mathbf{3}$ are more typical of the expected dimeric structures, considering 4 (Figure 4.5, Table 4.1). Both compounds crystallized in the monoclinic $\mathrm{P} 2{ }_{1} / \mathrm{c}$ space group with two molecules in the unit cell centred on inversion centres, where both molecules in the unit cell are identical. The $\mathrm{Cu}-\mathrm{Cu}$ distance are $\sim 2.5 \AA$, which is significantly closer than in $\mathbf{1}$. The copper geometry is very close to linear, contorting by 4. $7^{\circ}$ and $4.4^{\circ}$ to lengthen the $\mathrm{Cu}-\mathrm{Cu}$ bond for $\mathbf{2}$ and $\mathbf{3}$, respectively. The metallocycle cores are planar with the core N-C bonds all essentially equivalent $(1.33 \AA-1.34 \AA)$, suggesting that the inherent double bond character is delocalized between these two bonds. The parallelogram outlined by the four nitrogens of the metallocycle is more skewed in 2 than $\mathbf{3}\left(\mathrm{N} 2 \mathrm{~A}-\mathrm{N} 1-\mathrm{N} 2\right.$ angles; $95.0^{\circ}$ for $\mathbf{2}, 93.6^{\circ}$ for $\left.\mathbf{3}\right)$ which is a result of similar steric bulk provided by the pyrroline and imino groups in $\mathbf{3}$. 

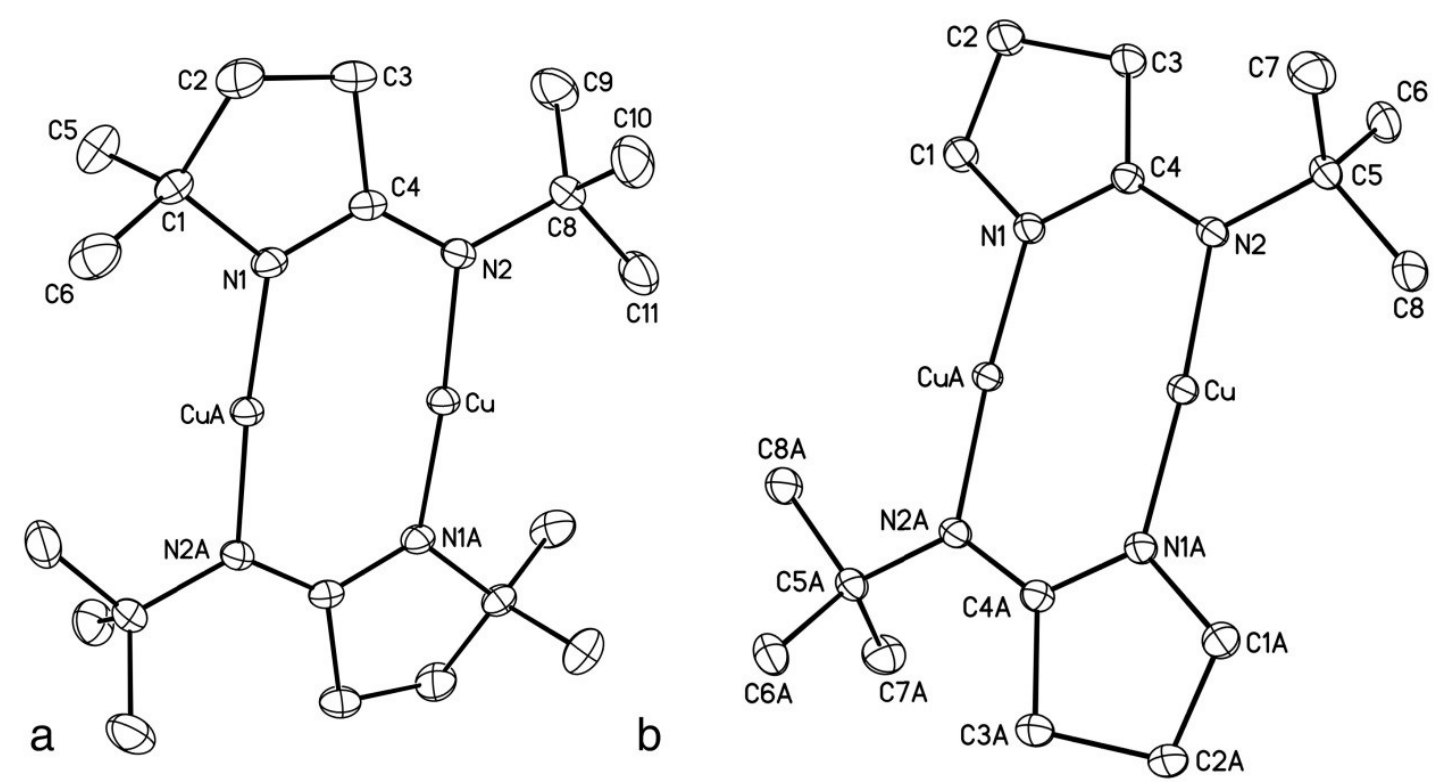

Figure 4.5 Structure of (a) 3 and (b) 4, shown with the hydrogens removed for clarity. The thermal ellipsoids are at $30 \%$.

The thermolyses of $\mathbf{1}-\mathbf{2}$ were quite encouraging. Compound $\mathbf{1}$ showed low residual mass using TGA, and a single feature in weight loss, suggesting that the species volatilized easily. This low residual mass was surprising. Not only does this compound possess $\beta$-hydrogen atoms for abstraction, but the tetrameric structure was expected to yield a lower vapour pressure from increased molecular mass. It is possible that there exists an oligomeric equilibrium that allows $\mathbf{1}$ to volatilize as a lower order oligomer (mass spectral analysis shows a dimer), thus providing a larger vapour pressure at lower temperature. This also explains why $\mathbf{1}$ has a similar onset of volatilization to the dimeric compounds 3 and $\mathbf{4}$.

Compound 2 showed more complex behaviour. The residual mass was $28.1 \%$, which was close to the percent mass of copper (31.3\%) in this compound. The residual mass was confirmed to be metallic copper by powder X-ray diffraction, suggesting that $\mathbf{2}$ undergoes a low temperature reduction to produce $\mathrm{Cu}(0)$. This has previously been observed in the case of both 4 and the copper amidinates. ${ }^{4}$ Due to the presence of the 
exocyclic pyrrolidine ring, the more favourable path for this thermal decomposition is $\beta$ hydrogen elimination rather than CDI deinsertion. This can be rationalized considering the crystal structure of $\mathbf{2}$. The distortion of the dimer core and lack of steric protection exposes the copper and enables it to interact with the $\beta$-hydrogen atoms of neighbouring molecules. On the other hand, $\mathbf{3}$ showed excellent volatile characteristics. Compound $\mathbf{3}$ has an onset of volatility at $165^{\circ} \mathrm{C}$ at atmospheric pressure, with a residual mass of $<1 \%$ of the sample mass. It could also be quantitatively sublimed at $130^{\circ} \mathrm{C}$ at a pressure of 20 mtorr.

These compounds were also investigated by a thermal stress test using TGA. Essentially, a compound is measured using the same temperature ramp rate $\left(10^{\circ} \mathrm{C} / \mathrm{min}\right)$ but using different sample masses. Thus, more sample would see higher temperatures due to the kinetics of thermolysis or volatilization. This valuable test can be used to gauge the behaviour of the compound with respect to thermal handling during a deposition process, whether in the source bubbler or during the volatilization and entrainment to the deposition zone. A good example of this is the thermal stress test of $\mathbf{1}$ (Figure 4.6b). Compound $\mathbf{1}$ was shown to have a higher residual mass as the initial pan loading of the TG was increased. This indicated that it underwent decomposition as more compound was exposed to higher temperature. Compound $\mathbf{3}$ (Figure 4.6a) was seen to undergo no decomposition as pan loading was increased, demonstrating a superior thermal stability. 

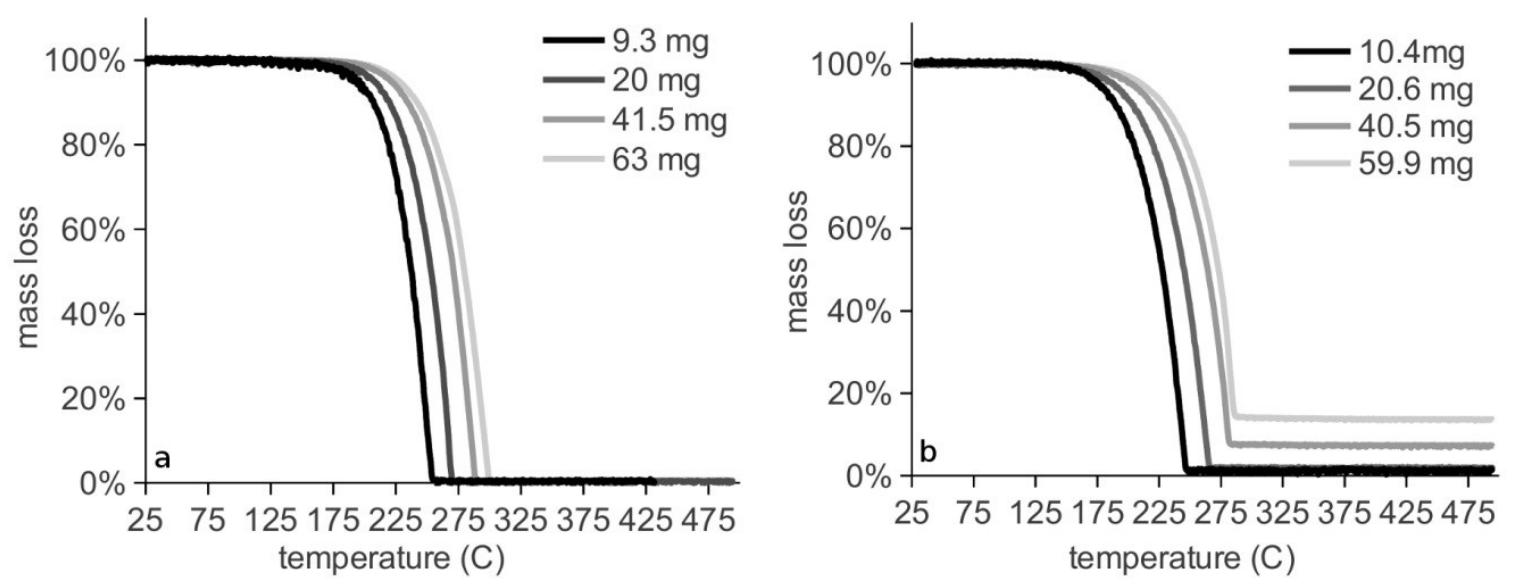

Figure 4.6 Thermal stress test of compounds (a) 3 showing no change in residual mass with increasing sample mass, and (b) 1, showing increasing residual mass with increasing initial sample mass.

The residual mass trends of each compound $\mathbf{1}-\mathbf{4}$ can be seen in Figure 4.7. It should be noted that the top sample mass that could be accommodated was about $65 \mathrm{mg}$; this filled the sample pan in all cases. With the exception of $\mathbf{1}$, the stress trends are quite linear with an $\mathrm{R}^{2}$ of 0.99 . Compound $\mathbf{1}$ showed a higher deviation from linearity with an $\mathrm{R}^{2}$ of 0.97 . This might be due to a higher preponderance of a dimeric rather than tetrameric species at higher temperature, caused by a shift in oligomer equilibrium.

Compounds 1 and $\mathbf{4}$ show the most stress, deviating from a negligible residual mass at low loadings to quite high residual masses (13.7\% for 1 and $9.0 \%$ for 4$)$ at high loadings. It is not surprising that these two compounds show similar thermal stress, since they both bear an isopropyl group for $\beta$-hydrogen elimination although CDI deinsertion cannot be ruled out in the case of 4 . 


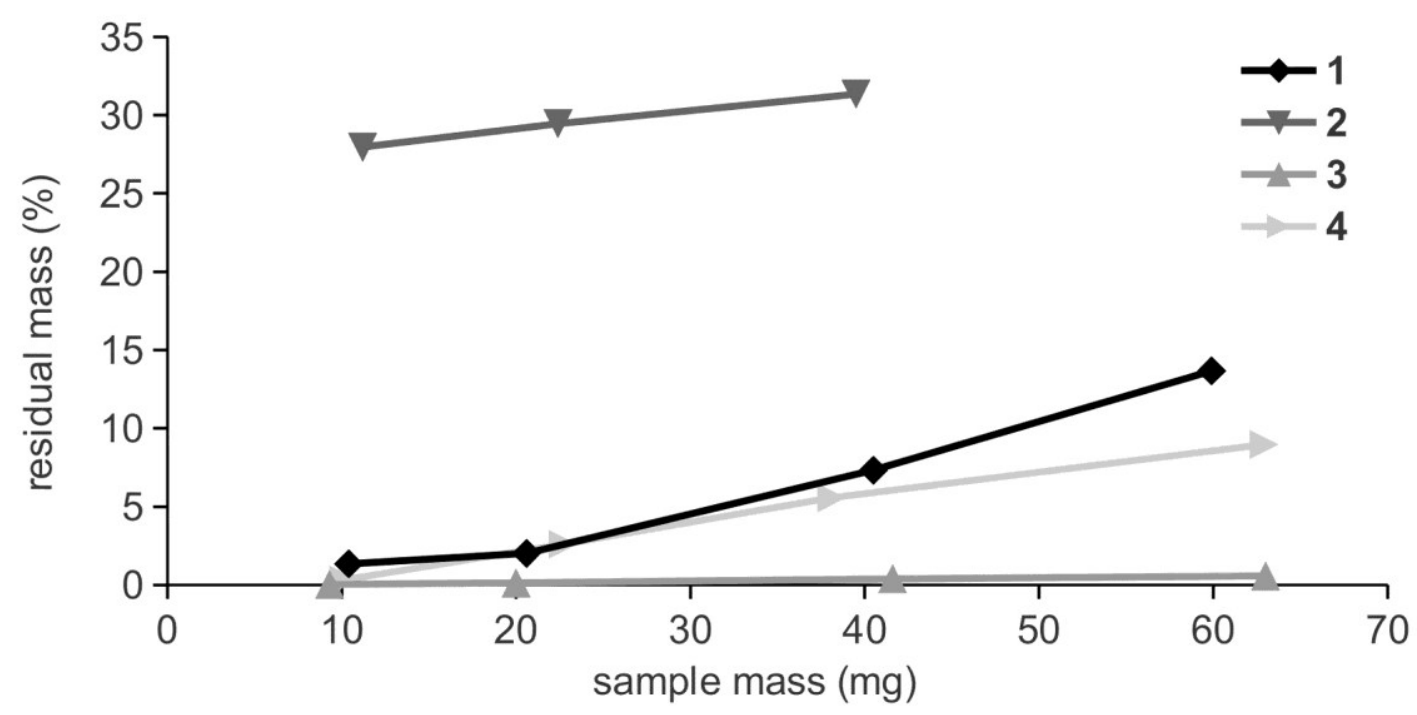

Figure 4.7 Thermal stress test trends for compounds 1-4. The lines are provided to guide the eye

Compound $\mathbf{2}$ again showed poor thermal behaviour with very high residual masses. Indeed, the highest loading was not attempted for 2 since the $39.5 \mathrm{mg}$ of sample gave $31.35 \%$ residual mass, which is the mass percent of copper in that compound $(31.32 \%)$. This was not surprising, given the high residual mass seen previously. This reactivity stands out due to the fact that $\mathbf{2}$ can be considered of intermediate reactivity with respect to number of $\beta$-hydrogens within this family. However, it has a dimeric structure (unlike 1, which exists in the solid as a tetramer) and so is less sterically protected at the copper centres. This would lend it worse thermal stability.

Compound 3 showed very good thermal stress resistance, rising only to $0.56 \%$ residual mass at $63.0 \mathrm{mg}$ sample mass. This supports our hypothesis that 3 will be the most thermally stable of this series of compounds due to its lack of reactive hydrogens and the robust nature of the core of this ligand with respect to CDI deinsertion.

Using TGA, the evaporation kinetics were evaluated for $\mathbf{1}-\mathbf{4}$ (Figure 4.8). Compound 4 showed the highest evaporation at the lowest temperatures. Compounds 1 


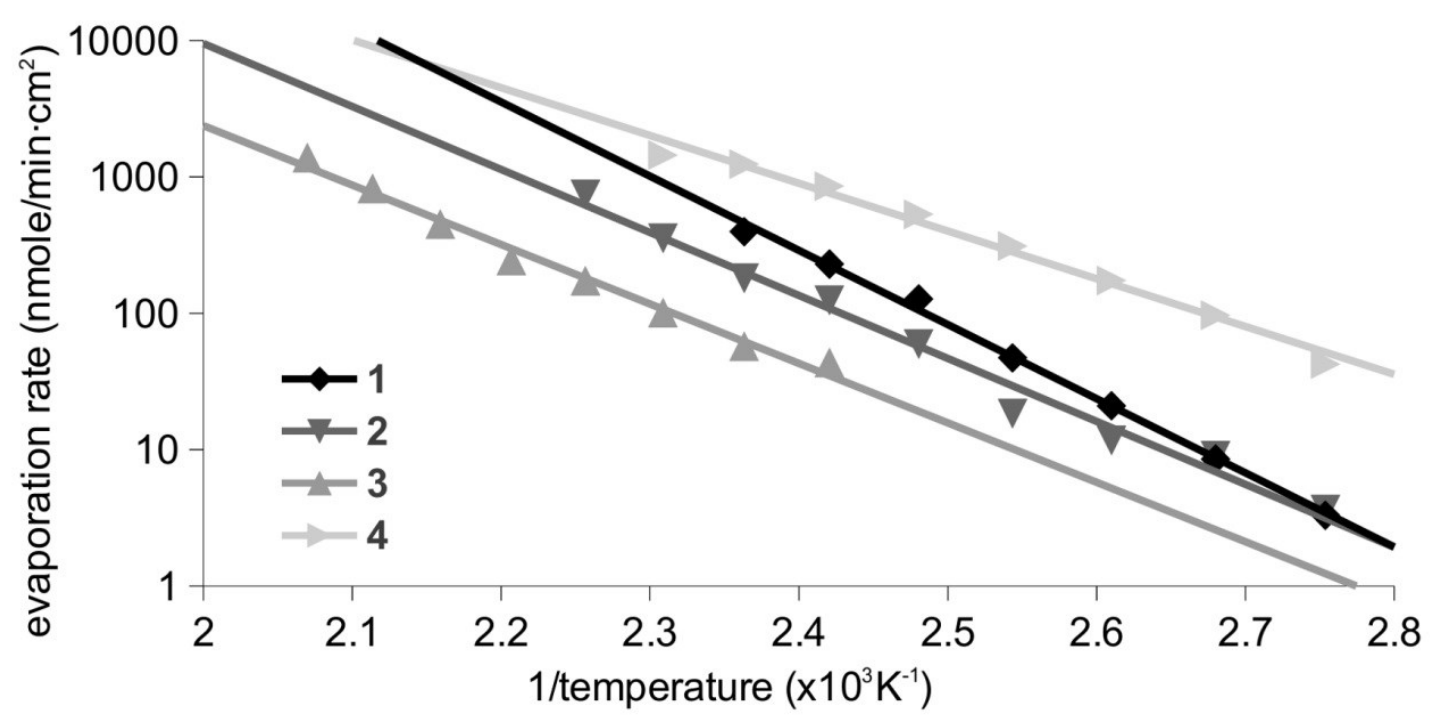

Figure 4.8 Evaporation kinetics of 1-4 by TGA.

and $\mathbf{2}$ showed similar evaporation kinetics within the temperatures studied. This is unsurprising since $\mathbf{1}$ and $\mathbf{2}$ are expected to both volatilize as dimers and have similar molecular masses (a mass difference of only two methyl groups). Surprisingly, $\mathbf{3}$ showed the slowest evaporation kinetics even though it has a mass close to that of $\mathbf{4}$. It is possible that the measured evaporation kinetics of the copper(I) iminopyrrolidinates are slower than 4 due to the more rigid framework of the iminopyrrolidinate ligand. The rigidity of the ligand would cause a compact, planar structure and allow for stronger intermolecular attraction within the solid. Additionally, the copper(I) iminopyrrolidinates would be expected to have less entropy gain during volatilization than 4 : the rigid ligand has fewer bonds that are free to rotate in the gas phase than in the solid state, compared to the relatively less constrained guanidinate of $\mathbf{4}$. From the isothermal TGA and using the Langmuir equation, ${ }^{13}$ the temperatures at which 1 Torr of vapour pressure was obtained was estimated to be $167^{\circ} \mathrm{C}, 173^{\circ} \mathrm{C}, 204^{\circ} \mathrm{C}$, and $138^{\circ} \mathrm{C}$ for $\mathbf{1}-4$, respectively..$^{14}$ 
To further illuminate the thermal chemistry of these compounds, they were each sealed in heavy walled NMR tubes and heated in an oven at $165^{\circ} \mathrm{C}$ over a period of days. An ${ }^{1} \mathrm{H}$ NMR was collected each day to observe their thermal decomposition (Figure 4.9, Table 4.2). Compound 4 revealed decomposition to produce diisopropylcarbodiimide, as previously seen. ${ }^{6}$ The decomposition of 4 that followed first order decomposition kinetics and had a calculated half-life of 33.8 hours. The parent guanidine was also observed as a product of thermolysis and its origin of production is discussed below.

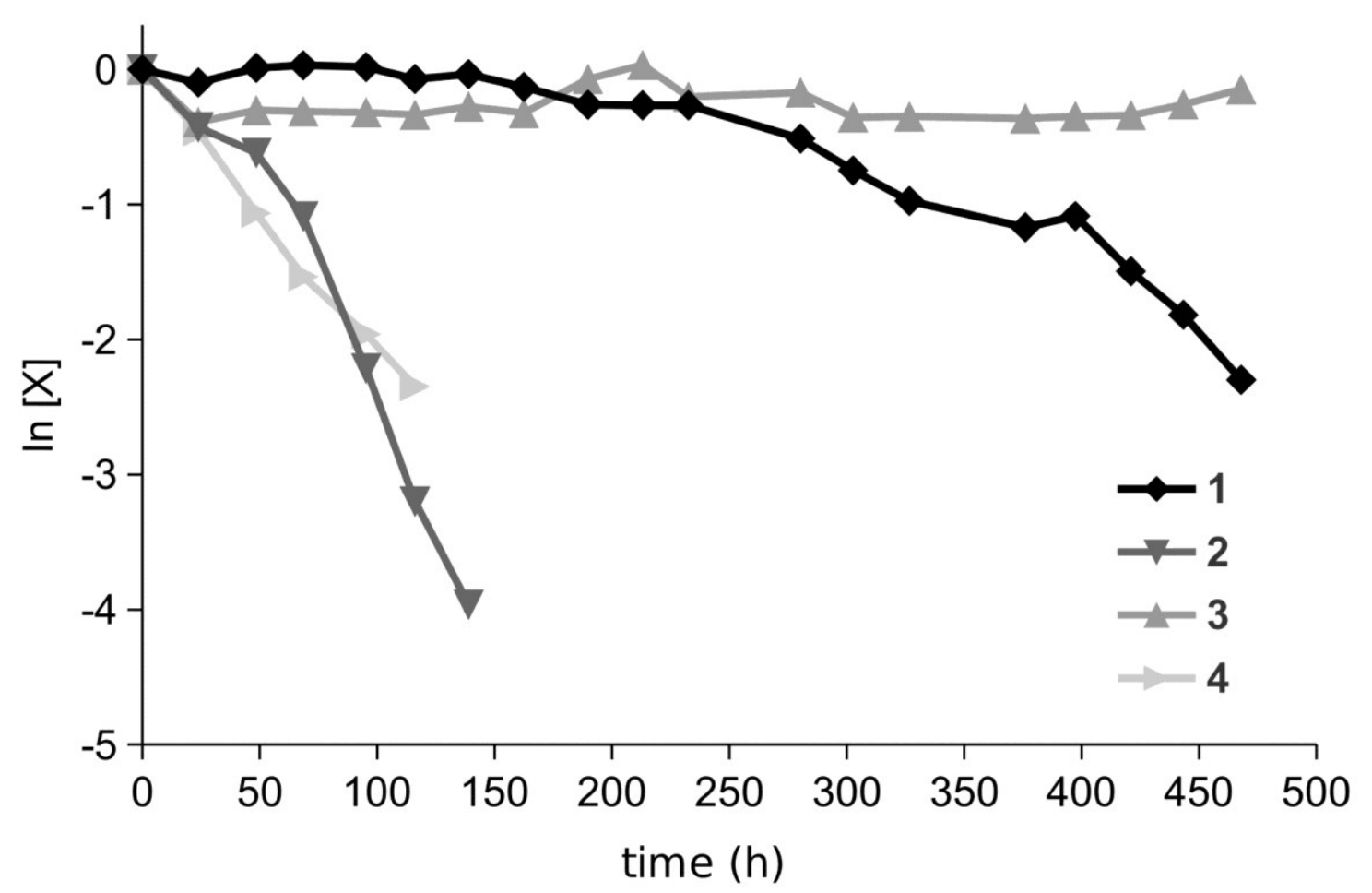

Figure 4.9 Thermal decomposition of $1-4$ in $\mathrm{C}_{6} \mathrm{D}_{6}$ at $165^{\circ} \mathrm{C}$ over 21 days.

Compound 1 showed much better thermal behaviour than $\mathbf{4}$, as was expected from the TG data. The compound decomposed following first order kinetics with a half-life of 181.1 hours, and there was very obvious plating of copper on the NMR tube walls. The main byproduct from the thermolysis was the parent ligand, isopropyliminopyrrolidine. There was no evidence of CDI nor any evidence of oxidized ligand. There was an 
obvious flocculant in the NMR tube after the thermolysis; this might be the oxidized ligand wherein a proton was lost. Compound $\mathbf{2}$ showed similar decomposition to $\mathbf{1}$, but much more quickly $\left(\mathrm{t}_{1 / 2}=23.7\right.$ hours). The NMR tube was again obscured with plated $\mathrm{Cu}(0)$ and had a flocculant precipitate, and the ${ }^{1} \mathrm{H}$ NMR showed free, protonated ligand. It is possible that since $\mathbf{1}$ forms a tetramer, this imparts thermal stability to the compound, perhaps because the tetramer has to dissociate to a smaller oligomer in order to thermalise. This would also account for the relatively poor linear fit to first order kinetics: if the tetramer forms at higher concentration, then there would be a slightly higher rate of decomposition as the overall concentration of $\mathbf{1}$ dropped and the dimertetramer equilibrium began to favour the dimer.

Table 4.2 Kinetic data for the thermal decomposition of $\mathbf{1}-\mathbf{4}$ at $165^{\circ} \mathrm{C}$. The "correlation coefficient" refers linear fit in Figure 4.9.

\begin{tabular}{cccc}
\hline Compound & Rate Constant & Calculated Half-life & Correlation Coefficient \\
\hline $\mathbf{1}$ & $1.06 \times 10^{-6}$ & 181.1 & 0.902 \\
$\mathbf{2}$ & $8.14 \times 10^{-6}$ & 23.7 & 0.944 \\
$\mathbf{3}$ & & no measurable decomposition & \\
$\mathbf{4}$ & $5.69 \times 10^{-6}$ & 33.8 & 0.995 \\
\hline
\end{tabular}

The full mechanism that produces the protonated ligand remains unclear. We propose $\beta$-hydrogens as the most likely source for protons for the origin of free ligand. Surface work by Gordon and Zaera ${ }^{4}$ found that butene elimination occurs from copper(I)$\mathrm{N}, \mathrm{N}$-di-secbutylacetamidinate leaving an N-H moiety, which might be another source for protons. However, no ${ }^{1} \mathrm{H}$ signals were observed in the NMR tube thermolysis experiment that suggest a similar mechanism occurring here. Evidence for disproportionation of $\mathrm{Cu}(\mathrm{I})$ acetamidinates has also been demonstrated in surface studies on $\mathrm{SiO}_{2}$ substrates. ${ }^{15}$ 
Since disproportionation of $\mathrm{Cu}(\mathrm{I})$ to $\mathrm{Cu}(\mathrm{II})$ and $\mathrm{Cu}(0)$ is a common thermolysis route for $\mathrm{Cu}(\mathrm{I})$ compounds, we offer the following insight. We observed a blue colour imparted to 2 during the solution based ${ }^{1} \mathrm{H}$ NMR thermolysis study (Figure 4.9), suggesting the formation of a $\mathrm{Cu}$ (II) species. Attempts to isolate a $\mathrm{Cu}$ (II) compound by crystallization failed and resulted only in the isolation of crystals of $\mathbf{2}$ with a blue impurity. Interestingly, we previously had prepared oxidized guanidine (i.e. the product of $\beta$ hydrogen elimination) from the reaction between guanidine and a $\mathrm{Cu}(\mathrm{II})$ compound. $^{7}$ Therefore, a $\mathrm{Cu}(\mathrm{II})$ intermediate might be involved in $\beta$-hydride abstraction thermolysis for this class of compound. We are continuing to study the thermolysis mechanisms of these compounds to gain a fuller understanding of this complicated thermal behaviour.

Compound 3 showed no decomposition at $165^{\circ} \mathrm{C}$ over 21 days. It was expected that its ligand (with no $\beta$-hydrogens and without the CDI deinsertion pathway) would impart the best thermal stability, however this marked difference was surprising. To further explore this, $\mathbf{3}$ was subjected to a rigorous surface study. This study used a combination of solid-state nuclear magnetic resonance (SS-NMR) and high-resolution NMR (HR-NMR) to determine the initial monolayer and surface chemistry of $\mathbf{3}$ on highsurface area silica. SS-NMR has been shown to be a useful tool in analysis of initial adsorption complexes on high-surface area substrates. ${ }^{16,17}$

To test compound $\mathbf{1}$ for vapour phase stability, it was heated in a bubbler at $160^{\circ} \mathrm{C}$ and the evolved vapour was entrained over high surface area silica for 17 hours to saturate the silica with a chemisorbed monolayer. Characterization was undertaken using ${ }^{1} \mathrm{H} /{ }^{13} \mathrm{C}$ cross-polarized magic-angle spinning solid state NMR, and there was only physisorbed species detected up to $200{ }^{\circ} \mathrm{C}$. At a deposition temperature of 275 
${ }^{\circ} \mathrm{C}$, solid state ${ }^{13} \mathrm{C}$ NMR showed signals attributable to the copper precursor (4.10a, top trace). These signals were similar to the high resolution ${ }^{13} \mathrm{C}$ NMR of $\mathbf{1}$ in deuterated benzene (Figure 4.10a, bottom trace). There was excellent corroboration between the solution NMR and the solid-state NMR. Although this suggested that the precursor chemisorbed "whole" at the surface, literature suggests that the alkyl group at the chelate position might be lost at high temperatures. ${ }^{4}$ The line width of the solid-state NMR did not permit unambiguous identification of the tertbutyl moiety, so another experiment was needed.

The silica with the deposited monolayer was rinsed with $\mathrm{D}_{2} \mathrm{O}$ to etch off the surface species, and a high resolution ${ }^{1} \mathrm{H}$ NMR was collected (Figure 4.10b, top trace), showing a singular surface species. Although the chemical shifts were different because of the necessity of using different solvents, the integration of the peaks for the surface species showed loss of the tertbutyl group: integrations of 2.1, 2.0, and 5.7 for the peaks at $2.94 \mathrm{ppm}, 2.07 \mathrm{ppm}$, and $1.36 \mathrm{ppm}$ respectively. The ${ }^{1} \mathrm{H}$ NMR of the ligand in deuterated chloroform clearly showed a singlet for the tertbutyl group (4.10b, bottom trace) with integration ratios of 2.0, 2.0, 9.4, and 6.1 for the peaks at $2.42 \mathrm{ppm}, 1.57 \mathrm{ppm}, 1.32 \mathrm{ppm}$, and $1.18 \mathrm{ppm}$ respectively. As well, ${ }^{29} \mathrm{Si}$ SSNMR of the silica showed no additional signals for silicon beyond what was detected for pure silica, suggesting nucleation did not occur at a silicon atom. This is reasonable, considering that the most likely nucleation point for this precursor on silica surface is at the oxygen of a silanol group. Given these data, nucleation likely occurs through the copper atom to a surface oxygen, and subsequently undergoes butene elimination (Figure 4.10c). This elimination occurs at $300{ }^{\circ} \mathrm{C}$ higher in 
temperature than shown for the copper amidinate on metallic surfaces, ${ }^{4}$ and highlights the excellent thermal stability designed into this ligand.

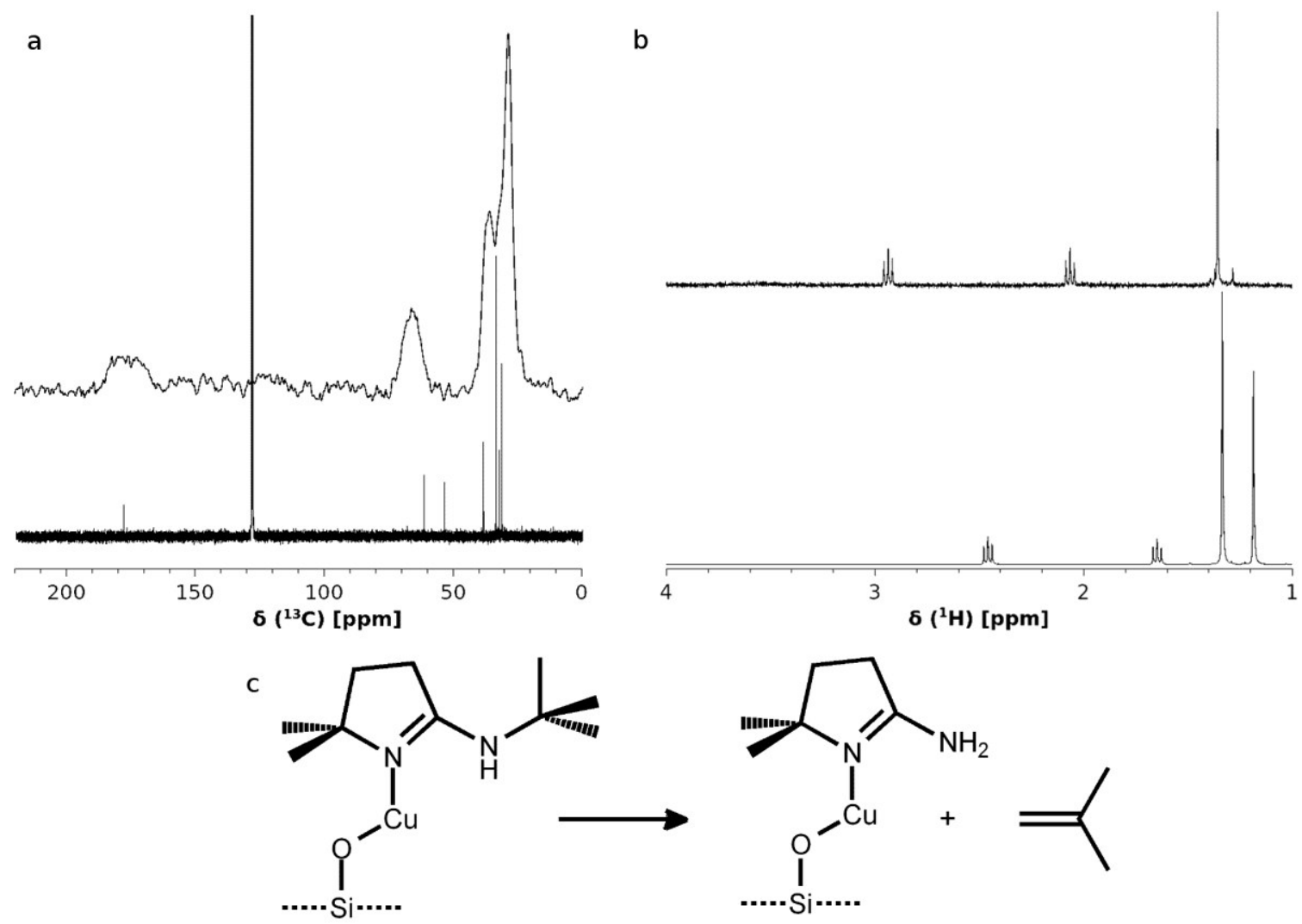

Figure 4.10 Reactivity of 1 with silica: (a) shows the $\mathrm{SS}-{ }^{13} \mathrm{C}$ NMR of silica after deposition of 1 at $275^{\circ} \mathrm{C}$ (top trace) compared to the $\mathrm{HR}-{ }^{13} \mathrm{C}$ NMR of $\mathbf{1}$ in deuterated benzene (bottom trace). (b) shows the HR- ${ }^{1} \mathrm{H}$ NMR of the surface species etched from the silica with $\mathrm{D}_{2} \mathrm{O}$ (in $\mathrm{D}_{2} \mathrm{O}$; top trace) compared to the HR- ${ }^{1} \mathrm{H}$ NMR of 1 in $\mathrm{CDCl}_{3}$ (bottom trace). (c) shows the proposed nucleation and chemisorption of $\mathbf{1}$ at a silanol group on a silica surface.

\subsection{Conclusion}

A series of copper(I) iminopyrrolidinates were synthesized and evaluated by thermogravimetric analysis and in $\mathrm{C}_{6} \mathrm{D}_{6}$ solutions by ${ }^{1} \mathrm{H}$ NMR to determine their thermal stability and suitability as precursors in CVD processes. The employed asymmetric, cyclic amidinate ligand proved to impart excellent thermal stability when no $\beta$-hydrogens 
were present. A direct comparison of the number of $\beta$-hydrogens to thermal stability was complicated by the tetrameric structure of $\mathbf{1}$, which was thought to influence thermal stability.

A valuable thermal stress test by TGA was devised and provided complimentary thermal stability trends to solution based thermolysis studies. Advantages of the thermal stress test by TGA was faster data collection, and use of conditions more pertinent to CVD processes. The solution based thermolysis studies identified the parent amidine and copper metal as thermolysis products of $\mathbf{1}$ and $\mathbf{2}$. The amidines were produced through an intramolecular hydrogen abstraction and a $\beta$-hydride abstraction mechanism was proposed. Compound $\mathbf{3}$ showed negligible decomposition in both the TGA and solution experiments, and is an excellent candidate for copper deposition experiments.

Gas-phase experiments suggests that 3 is stable at temperatures $>200^{\circ} \mathrm{C}$. Surface analysis suggested that $\mathbf{3}$ undergoes an alkene elimination from the chelating nitrogen atom during chemisorption on silica at $275^{\circ} \mathrm{C}$.

\subsection{Experimental}

General Considerations: All manipulations involving the synthesis and handling of copper(I) compounds were performed in a nitrogen filled drybox. All manipulations involving the synthesis of the pyrrole and the pyrrolidine were performed in air. The chemicals $\mathrm{CuCl}, 2.5 \mathrm{M}$ BuLi in hexanes, tert-butyl amine, and diethyl sulfate were purchased from Aldrich Chemical Company and used as received. All solvents used in manipulation of copper(I) compounds were ACS grade and purified from an Mbraun Solvent Purifier System. All other solvents were ACS grade and used as received. Nuclear Magnetic Resonance was done on $300 \mathrm{MHz}$ Avance 3 and $400 \mathrm{MHz}$ Bruker 
AMX. Canadian Microanalytical Service Ltd. performed combustion analyses. Therm al gravimetric analysis was performed on a TA Instruments Q50 apparatus located in an MBraun Labmaster 130 Dry box under a nitrogen atmosphere. 5, 5-dimethyl-2pyrrolidone was prepared by literature procedures. ${ }^{18}$ Tert-butyl ammonium chloride was prepared by addition of $\mathrm{HCl}_{\text {(aq) }}$ to tert-butyl amine in ethanol followed by solvent removal. Isopropyl-iminopyrrolidine and tert-butyl iminopyrrolidine were prepared by literature procedures. ${ }^{9}$

Copper(I) isopropyl-iminopyrrolidinate (1): Isopropyl-iminopyrrolidine $(5.11 \mathrm{~g}$, $40.49 \mathrm{mmol}$ ) was partially dissolved in $60 \mathrm{~mL}$ of $\mathrm{Et}_{2} \mathrm{O}$ and cooled to $0{ }^{\circ} \mathrm{C}$ in an ice bath. 16.2 $\mathrm{mL}$ of butyl lithium was added dropwise and a suspension formed after stirring for 2 h. In a separate flask, $\mathrm{CuCl}(4.2 \mathrm{~g}, 42.42 \mathrm{mmol})$ was suspended in $60 \mathrm{~mL}$ of THF and cooled to $0{ }^{\circ} \mathrm{C}$ in an ice bath. The suspension of lithium isopropyl-iminopyrrolidine was added via cannula to the cooled suspension of $\mathrm{CuCl}$ and was then allowed to warm to room temperature and stirred overnight. The volatiles were removed from the reaction flask by reduced pressure and the remaining solid was stirred in $100 \mathrm{~mL}$ of toluene for 10 minutes. The cloudy solution was filtered and the clear filtrate was concentrated under vacuum and kept at $-35^{\circ} \mathrm{C}$ for 2 days. A mass of small, needle crystals was collected by filtration, washed with pentane and then dried under vacuum to afford $5.03 \mathrm{~g}, 65.8 \%$. Mp $138^{\circ} \mathrm{C} .{ }^{1} \mathrm{H}$ NMR $\left(400 \mathrm{MHz}, \mathrm{C}_{6} \mathrm{D}_{6}\right): \delta 3.49, \delta 3.24\left(\mathrm{~m}, 1 \mathrm{H}, \mathrm{NCH}\left(\mathrm{CH}_{3}\right)_{2}\right) ; \delta 3.34, \delta 3.26($ t, $2 \mathrm{H}, \mathrm{NCH}_{2} \mathrm{CH}_{2} \mathrm{CH}_{2} \mathrm{C}$ ); 2.04 (t, $2 \mathrm{H}, \mathrm{NCH}_{2} \mathrm{CH}_{2} \mathrm{CH}_{2} \mathrm{C}$ ); $\delta 1.58$ (quintet, $2 \mathrm{H}$, $\left.\mathrm{NCH}_{2} \mathrm{CH}_{2} \mathrm{CH}_{2} \mathrm{C}\right) ; \delta 1.487, \delta 1.304, \delta 1.208, \delta 1.158\left(\mathrm{~d}, 6 \mathrm{H}, \mathrm{NCH}\left(\mathrm{CH}_{3}\right) 2\right) .{ }^{13} \mathrm{C} \mathrm{NMR}(300$ $\left.\mathrm{MHz}, \mathrm{C}_{6} \mathrm{D}_{6}\right) \delta 179.84, \delta 175.38, \delta 54.19, \delta 53.30, \delta 53.15, \delta 51.19, \delta 50.48, \delta 50.00, \delta$ $29.02, \delta 28.69, \delta 28.65, \delta 27.70, \delta 27.55, \delta 27.39, \delta 27.36, \delta 24.99, \delta 24.72, \delta 24.64$. 
Combustion analysis, found (calculated): C, 44.63(44.55); H, 7.11(6.94); N, 14.84(14.84).

Copper(I) tertbutyl-iminopyrrolidinate (2): Tert-butyl iminopyrrolidine (1.72 g, $12.26 \mathrm{mmol}$ ) was dissolved in $60 \mathrm{~mL}$ of THF. $4.9 \mathrm{~mL}$ of $2.5 \mathrm{M}$ butyl lithium was added and the solution was stirred for $2 \mathrm{~h} . \mathrm{CuCl}(1.25 \mathrm{~g}, 12.62 \mathrm{mmol})$ was added in one portion and the suspension was stirred overnight. Volatiles were removed under reduced pressure and the remaining solid was taken up in THF and filtered. The filtrate was concentrated and then kept at $-35^{\circ} \mathrm{C}$ for 1 day. Colourless, block crystals were collected by decanting the solution, washing with pentane, and then drying under vacuum; obtained $1.59 \mathrm{~g}, 63.9$ \%. Mp $148{ }^{\circ} \mathrm{C} .{ }^{1} \mathrm{H}$ NMR $\left(300 \mathrm{MHz}, \mathrm{C}_{6} \mathrm{D}_{6}\right): \delta 3.25$ (t, $\left.2 \mathrm{H}, \mathrm{NCH}_{2} \mathrm{CH}_{2} \mathrm{CH}_{2} \mathrm{C}\right), \delta 2.19(\mathrm{t}, 2 \mathrm{H}$, $\mathrm{NCH}_{2} \mathrm{CH}_{2} \mathrm{CH}_{2} \mathrm{C}$ ), $\delta 1.63$ (quintet, $2 \mathrm{H}, \mathrm{NCH}_{2} \mathrm{CH}_{2} \mathrm{CH}_{2} \mathrm{C}$ ), $\delta 1.32$ (s, $\left.9 \mathrm{H}, \mathrm{NC}\left(\mathrm{CH}_{3}\right)_{3}\right) \cdot{ }^{13} \mathrm{C}$ NMR (300 MHz, $\left.\mathrm{C}_{6} \mathrm{D}_{6}\right): \delta 180.01\left(\mathrm{NCH}_{2} \mathrm{CH}_{2} \mathrm{CH}_{2} \mathrm{C}\right), \delta 52.45\left(\mathrm{NCH}_{2} \mathrm{CH}_{2} \mathrm{CH}_{2} \mathrm{C}\right), \delta 33.72$ $\left(\mathrm{NC}\left(\mathrm{CH}_{3}\right)_{3}\right), \delta 33.26\left(\mathrm{NC}\left(\mathrm{CH}_{3}\right)_{3}\right), \delta 31.64\left(\mathrm{NCH}_{2} \mathrm{CH}_{2} \mathrm{CH}_{2} \mathrm{C}\right), \delta 26.33\left(\mathrm{NCH}_{2} \mathrm{CH}_{2} \mathrm{CH}_{2} \mathrm{C}\right)$. Combustion analysis, found (calculated): C, 47.08(47.39); H, 7.49(7.46); N, 13.69(13.82)

Copper(I) tertbutyl-imino-2,2-dimethylpyrrolidinate (3): Tertbutyl-imino-2,2dimethylpyrrolidine $(5.103 \mathrm{~g}, 13.07 \mathrm{mmol})$ was dissolved in $170 \mathrm{~mL}$ of toluene and the solution was cooled on an ice bath. $12.13 \mathrm{ml}$ of $2.5 \mathrm{M} \mathrm{BuLi}$ was added dropwise. The solution warmed to r.t as it stirred overnight. $\mathrm{CuCl}(3.26 \mathrm{~g}, 32.9 \mathrm{mmol})$ was added and stirring continued for $18 \mathrm{~h}$. The cloudy, light brown solution was filtered and the filter cake was extracted with $4 \times 20$ of THF. Volatiles were stripped from the combined toluene and THF solutions to afford an off-white solid. The solid was sublimed at $130^{\circ} \mathrm{C}$ at $40 \mathrm{mtorr}$ and $5.795 \mathrm{~g}, 82.7 \%$, of a white solid was obtained. $\mathrm{Mp}>270{ }^{\circ} \mathrm{C} .{ }^{1} \mathrm{H}$ NMR (300 MHz, $\left.\mathrm{C}_{6} \mathrm{D}_{6}\right): \delta 2.428\left(\mathrm{t}, 2 \mathrm{H}, \mathrm{NC}\left(\mathrm{CH}_{3}\right)_{2} \mathrm{CH}_{2} \mathrm{CH}_{2} \mathrm{C}\right), \delta 1.581$ (t, $2 \mathrm{H}$, 
$\left.\mathrm{NC}\left(\mathrm{CH}_{3}\right)_{2} \mathrm{CH}_{2} \mathrm{CH}_{2} \mathrm{C}\right), \delta 1.328\left(\mathrm{~s}, 9 \mathrm{H}, \mathrm{NC}\left(\mathrm{CH}_{3}\right)_{3}\right), \delta 1.196\left(\mathrm{~s}, 6 \mathrm{H}, \mathrm{NC}\left(\mathrm{CH}_{3}\right)_{2} \mathrm{CH}_{2} \mathrm{CH}_{2} \mathrm{C}\right)$.

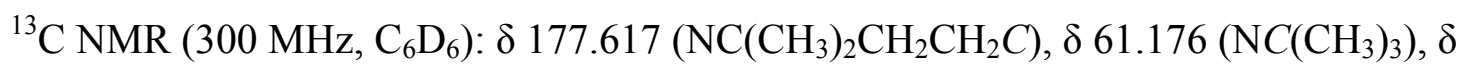
$53.332\left(\mathrm{NC}\left(\mathrm{CH}_{3}\right)_{2} \mathrm{CH}_{2} \mathrm{CH}_{2} \mathrm{C}\right), \delta 38.095\left(\mathrm{NC}\left(\mathrm{CH}_{3}\right)_{2} \mathrm{CH}_{2} \mathrm{CH}_{2} \mathrm{C}\right), \delta 33.215\left(\mathrm{NC}\left(\mathrm{CH}_{3}\right)_{3}\right), \delta$ $31.931\left(\mathrm{NC}\left(\mathrm{CH}_{3}\right)_{2} \mathrm{CH}_{2} \mathrm{CH}_{2} \mathrm{C}\right), \delta 30.940\left(\mathrm{NC}\left(\mathrm{CH}_{3}\right)_{2} \mathrm{CH}_{2} \mathrm{CH}_{2} \mathrm{C}\right)$. Combustion analysis, found (calculated): C, 52.44(52.04); H, 8.36(8.30); N, 12.27(12.14).

5-ethoxy-3, 4-dihydro-2,2-dimethyl-2H-pyrrole: Similar to the literature preparation for 5-ethoxy-3, 4-dihydro-2H-pyrrole: ${ }^{19}$ 5, 5-dimethyl-2-pyrrolidone $(9.82 \mathrm{~g}$, $86.8 \mathrm{mmol})$ and diethyl sulfate $(13.38 \mathrm{~g}, 86.8 \mathrm{mmol})$ were added to a pressure vessel and heated for $24 \mathrm{~h}$ at $60{ }^{\circ} \mathrm{C}$. $\mathrm{Et}_{2} \mathrm{O}(30 \mathrm{ml})$ was added and the biphasic mixture was cool in an ice bath. A $25 \mathrm{ml}$ aqueous solution of $\mathrm{Na}_{2} \mathrm{CO}_{3}(9.20 \mathrm{~g}, 86.8 \mathrm{mmol})$ was added drop wise. The top organic layer was separated and extraction continued with $3 \times 40 \mathrm{ml}$ of $\mathrm{Et}_{2} \mathrm{O}$. The combined extractions were dried over $\mathrm{MgSO}_{4}$. The majority of $\mathrm{Et}_{2} \mathrm{O}$ was removed on a roto-vap followed by condensation of the remaining volatiles into a cold trap under reduced pressure thereby transferring remaining $\mathrm{Et}_{2} \mathrm{O}$ and product to the cold trap. $\mathrm{A}$

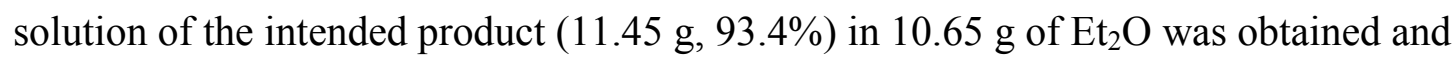
used without additional purification. ${ }^{1} \mathrm{H}$ NMR $\left(400 \mathrm{MHz}, \mathrm{CDCl}_{3}\right): \delta 4.16$ (q, $2 \mathrm{H}$, $\left.\mathrm{COCH}_{2} \mathrm{CH}_{3}\right), \delta 2.53\left(\mathrm{t}, 2 \mathrm{H}, \mathrm{NC}\left(\mathrm{CH}_{3}\right)_{2} \mathrm{CH}_{2} \mathrm{CH}_{2} \mathrm{C}\right), \delta 1.80\left(\mathrm{t}, 2 \mathrm{H}, \mathrm{NC}\left(\mathrm{CH}_{3}\right)_{2} \mathrm{CH}_{2} \mathrm{CH}_{2} \mathrm{C}\right), \delta$ $1.30\left(\mathrm{t}, 3 \mathrm{H}, \mathrm{COCH}_{2} \mathrm{CH}_{3}\right), \delta 1.23\left(\mathrm{~s}, 6 \mathrm{H}, \mathrm{NC}\left(\mathrm{CH}_{3}\right)_{2} \mathrm{CH}_{2} \mathrm{CH}_{2} \mathrm{C}\right) .{ }^{13} \mathrm{C} \mathrm{NMR}(400 \mathrm{MHz}$, $\left.\mathrm{CDCl}_{3}\right): \delta 170.05(\mathrm{NCO}), \delta 67.36\left(\mathrm{NC}\left(\mathrm{CH}_{3}\right)_{2}\right), \delta 63.67\left(\mathrm{OCH}_{2} \mathrm{CH}_{3}\right), \delta 36.73$

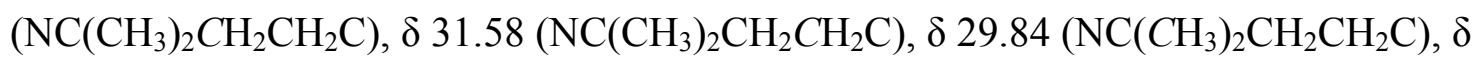
$14.41\left(\mathrm{OCH}_{2} \mathrm{CH}_{3}\right)$. HRMS (EI) $\mathrm{m} / z$ calcd for $\mathrm{C}_{8} \mathrm{H}_{15} \mathrm{NO} \mathrm{M}^{+} 141.1154$, found 141.1164 .

Tertbutyl-imino-2,2-dimethylpyrrolidine: In a pressure vessel, 5-ethoxy-3,4dihydro-2,2-dimethyl-2H-pyrrole (11.45 g, $81.1 \mathrm{mmol})$ in $10.65 \mathrm{~g}_{\mathrm{g}} \mathrm{Et}_{2} \mathrm{O}$, tert-butyl 
ammonium chloride ( $8.89 \mathrm{~g}, 81.1 \mathrm{mmol})$, and tert butyl amine $(11.86 \mathrm{~g}, 162.2 \mathrm{mmol})$ were dissolved in $80 \mathrm{~mL}$ of ethanol. The pressure vessel was heated to $130{ }^{\circ} \mathrm{C}$ for 2 days with stirring. The vessels was removed from heat and cooled to room temperature.

Volatiles were stripped under reduced pressure. The solid residue was dissolved in $80 \mathrm{~mL}$ of $\mathrm{H}_{2} \mathrm{O}$ and made basic with $40 \mathrm{~mL}$ of $6 \mathrm{M} \mathrm{NaOH}$. The aqueous solution was extracted with $3 \times 60 \mathrm{~mL}$ of $\mathrm{Et}_{2} \mathrm{O}$. The combined $\mathrm{Et}_{2} \mathrm{O}$ extractions were dried over $\mathrm{Na}_{2} \mathrm{SO}_{4}$ and then the solution was concentrated under reduced pressure to obtain $11.43 \mathrm{~g}$ of a light yellow liquid, $83.8 \% .{ }^{1} \mathrm{H}$ NMR (400 MHz, $\left.\mathrm{CDCl}_{3}\right): \delta 2.46\left(\mathrm{t}, 2 \mathrm{H}, \mathrm{NC}\left(\mathrm{CH}_{3}\right)_{2} \mathrm{CH}_{2} \mathrm{CH}_{2} \mathrm{C}\right), \delta$ $1.65\left(\mathrm{t}, 2 \mathrm{H}, \mathrm{NC}\left(\mathrm{CH}_{3}\right)_{2} \mathrm{CH}_{2} \mathrm{CH}_{2} \mathrm{C}\right), \delta 1.34\left(\mathrm{~s},\left(\mathrm{H}, \mathrm{NC}\left(\mathrm{CH}_{3}\right)_{3}, \delta 1.19(\mathrm{~s}, 6 \mathrm{H}\right.\right.$, $\left.\mathrm{NC}\left(\mathrm{CH}_{3}\right)_{2} \mathrm{CH}_{2} \mathrm{CH}_{2} \mathrm{C}\right){ }^{13} \mathrm{C} \mathrm{NMR}\left(400 \mathrm{MHz}, \mathrm{CDCl}_{3}\right): \delta 161.57(\mathrm{NCO}), \delta 69.03$ $\left(\mathrm{NC}\left(\mathrm{CH}_{3}\right)_{2}\right), \delta 50.81\left(\mathrm{NC}\left(\mathrm{CH}_{3}\right)_{3}\right), \delta 36.60\left(\mathrm{NC}\left(\mathrm{CH}_{3}\right)_{2} \mathrm{CH}_{2} \mathrm{CH}_{2} \mathrm{C}\right), \delta 34.62$ $\left(\mathrm{NC}\left(\mathrm{CH}_{3}\right)_{2} \mathrm{CH}_{2} \mathrm{CH}_{2} \mathrm{C}\right), \delta 29.85\left(\mathrm{NC}\left(\mathrm{CH}_{3}\right)_{2}\right), \delta 29.30\left(\mathrm{NC}\left(\mathrm{CH}_{3}\right)_{3}\right)$. HRMS (EI) $\mathrm{m} / z$ calcd for $\mathrm{C}_{10} \mathrm{H}_{20} \mathrm{~N}_{2} \mathrm{M}^{+}$168.1626, found 168.1618.

Crystallography: X-ray structural analysis for $\mathbf{1}-\mathbf{3}$ : Crystals were selected and mounted on plastic mesh using viscous oil flash-cooled to the data collection temperature. Data were collected on a Bruker-AXS APEX CCD diffractometer with graphite-monochromated Mo-K $\alpha$ radiation $(\lambda=0.71073 \AA)$. Unit cell parameters were obtained from 60 data frames, $0.3^{\circ} \omega$, from three different sections of the Ewald sphere. The systematic absences in the data and the unit cell parameters were uniquely consistent to $C c c a$ for $\mathbf{1}$ to $P 2_{1 / c}$ for $\mathbf{2}$ and $\mathbf{3}$. The data-sets were treated with SADABS absorption corrections based on redundant multiscan data.20 The structures were solved using direct methods and refined with full-matrix, least-squares procedures on $\mathrm{F}^{2}$. The compound molecules were each located on an inversion point in $\mathbf{2}$ and $\mathbf{3}$. Two symmetry unique but 
chemically identical molecules of the compound in $\mathbf{1}$ are each located on a two-fold rotation axis: in one case the two-fold axis is parallel to the $\mathrm{N}-\mathrm{Cu}-\mathrm{N}$ axis and in the other case the two-fold axis is perpendicular to the $\mathrm{N}-\mathrm{Cu}-\mathrm{N}$ axis bisecting the $\mathrm{Cu}$ atoms on the opposite distal positions of the tetracopper rhombus. One THF solvent molecule of crystallization per two tetrameric complexes in $\mathbf{1}$ was located severely disordered and treated as diffused contribution.21 One isopropyl group in $\mathbf{1}$ was located disordered with a 23/77 refined occupancy ratio. Chemically equivalent bond distances and angles in the disordered group were restrained to average values with equal atomic displacement atomic parameter contraints on equivalent atoms. All non-hydrogen atoms were refined with anisotropic displacement parameters. All hydrogen atoms were treated as idealized contributions. Atomic scattering factors are contained in the SHELXTL 6.12 program library. $^{20}$

Surface Exposure Experiments: The exposure experiments were performed in a home-built reactor. The reaction chamber consisted of a stainless steel ring support covered in 200 stainless steel mesh with a plug of glass wool to prevent loss of silica powder. The system had one inlet from a heated bubbler and one inlet for He (purity of 99.999 \%). All fittings used in this system were either CF or VCR to ensure a highvacuum seal. The system was leak checked using a gas thermal conductivity/ leak detector (Gow-Mac Instrument Co.) and an overpressure of He. For the exposure experiments, typically about $1 \mathrm{~g}$ of high surface area $\mathrm{SiO}_{2}$ powder (EP10X; PQ Corporation; $300 \mathrm{~m}^{2} / \mathrm{g}$ S.A.; $1.8 \mathrm{~cm}^{3} / \mathrm{g}$ P.V.; $24 \mathrm{~nm}$ P.S.; $100 \mu \mathrm{m}$ P.D.) was used. The powder was pre-treated in the reactor at $350{ }^{\circ} \mathrm{C}$ for $16 \mathrm{~h}$ under vacuum before exposure to the precursor. The reactor and lines were heated to temperature and allowed to equilibrate 
for 1-2 $\mathrm{h}$ before introduction of the precursor. The precursor (typically $0.6-0.8 \mathrm{~g}$ ) was then vaporized and transported to the substrate with the system under $10^{-4}$ Torr vacuum. The substrate was exposed to volatilized precursor for $17 \mathrm{~h}$ before the system was cooled to room temperature for handling. Both precursor and substrate were handled in inert atmosphere.

Characterization of Surface Species: Solid-state NMR experiments were performed at $4.7 \mathrm{~T}$ on a Bruker Avance III console. All spectra were obtained using a Bruker $7 \mathrm{~mm}{ }^{1} \mathrm{H} / \mathrm{X} / \mathrm{Y}$ probe. ${ }^{13} \mathrm{C}\left(v_{0}=50.3 \mathrm{MHz}\right)$ cross-polarization magic angle spinning (CP/MAS) experiments were collected at a spinning rate of $4.5 \mathrm{kHz}$ using a 3.4 $\mu$ s $90^{\circ}$ proton pulse with a contact time of 2 ms where the contact pulse was ramped on the ${ }^{1} \mathrm{H}$ channel. A relaxation delay of $2 \mathrm{~s}$ was sufficient to prevent saturation and typically total acquisition times were 16-30 h. Glycine was used as an external secondary reference for the ${ }^{13} \mathrm{C}$ chemical shift scale. Spectra were treated with $40 \mathrm{~Hz}$ line broadening during processing. All spectra were obtained with high power proton decoupling during acquisition.Samples were prepared for High Resolution NMR (HR-NMR) by adding a small amount, typically $40-50 \mathrm{mg}$, of modified silica powder to $2 \mathrm{~mL}$ of $\mathrm{D}_{2} \mathrm{O}$, agitating, and allowed to sit for $15 \mathrm{~min}$. The $\mathrm{D}_{2} \mathrm{O}$ solution was then decanted and studied via HRNMR. $\mathrm{d}_{4}$-TSP was used as an internal reference.

\subsection{References}

12011 Edition of the International Technology Roadmap for Semiconductors; http://www.itrs.net/Links/2011ITRS/Home2011.htm.

2 (a) Li, Z.; Rahtu, A.; Gordon, R. G..J. Electrochem. Soc. 2006, 153, C787, (b) Li, Z.; Barry, S. T.; Gordon, R. G. Inorg. Chem., 2005, 44, 1728. 
3 Krisyuk, V; Aloui, L.; Prud'homme, N.; Sysoev S.; Senocq, F.; Samélor, D.; Vahlas, C. Electrochem. Solid-State Lett. 2011, 14, D26.

4 (a) Ma, Q.; Guo, H.; Gordon, R. G.; Zaera, F. Chem. Mater. 2010, 22, 352, (b) Ma, Q.; Guo, H.; Gordon, R. G.; Zaera, F. Chem. Mater. 2011, 23, 3325.

5 Turgambaeva, A.; Prud'homme, N.; Krisyuk, V.; Vahlas, C. J. Nanosci. Nanotechnol. 2011, 11, 8198.

6 Coyle, J. P.; Monillas, W. H.; Yap, G. P. A.; Barry S. T. Inorg. Chem. 2008, 47, 683.

7 Coyle, J. P.; Johnson, P. A.; DiLabio, G. A.; Barry, S. T.; Muller, J. Inorg. Chem. 2010, 49, 2844.

8 Tsuda, T., Watanabe, K., Miyata, K., Yamamoto, H. and Saegusa, T. Inorg. Chem. 1981, 20, 2728.

9 Wasslen, Y. A.; Kurek, A.; Johnson, P. A.; Pigeon, T. C.; Monillas, W. H.; Yap, G. P. A.; Barry, S. T. Dalton Trans. 2010, 39, 9046.

10 Willcocks, A. M.; Robinson, T. P.; Roche, C.; Pugh, T.; Richards, S. P.; Kingsley, A. J.; Lowe, J. P.; Johnson, A. L. Inorg. Chem. 2012, 51, 246.

11 Cotton, F. A.; Daniels, L. M.; Feng, X.; Maloney, D. J.; Matonic, J. H.; Murilio, C. A. Inorg. Chim. Acta 1997, 256, 291.

12 (a) Abdou, H. E.; Mohamed, A. A.; Fackler, J. P. Jiang, X.; Bollinger, J. J. Cluster Sci. 2007, 18, 630, (b) Baik, M.-H. Lee, D. Chem. Commun. 2005, 1043, (c) Beck, J.; Strähle, J. Angew. Chem. Int. Ed. Engl. 1986, 25, 95.

13 Kunte, G. V.; Shivashanker, S. A.; Umarji, A. M. Meas. Sci. Tech. 2008, 19, 025704.

14 The 1 Torr values are corrected from our previous publication (Coyle et al. Inorg. Chem. 2013, 52, 910) because of a calculation error for the value of the sample pan surface area.

15 Dai, M.; Kwon, J.; Halls, M. D.; Gordon, R. G.; Chabal, Y. J. Langmuir 2010, 26, 3911. 
16 Haukka, S.; Lakomaa, E. L.; Root, A. J. Phys. Chem. 1993, 97, 5085.

17 Wasslen, Y. A.; Tois, E.; Haukka, S.; Kreisel, K. A.; Yap, G. P. A.; Halls, M. D.; Barry, S. T. Inorg. Chem. 2010, 49, 1976.

18 (a) Moffett, R. B. Organic Synthesis 1952, 32, 59. (b) Osby, J. O.; Ganem, B. Tetrahedron Lett. 1985, 26, 6413.

19 Beckman, U.; Eichberger, E.; Lindner, M.; Bongartz, M.; Kunz, P. C. Eur. J. Org. Chem. 2008, 24, 4139.

20 Sheldrick, G.M. Acta Cryst. 2008, A64, 112.

21 Spek, A. L. J. Appl. Cryst. 2003, 36, 7. 


\section{Chapter: Thermally Robust Gold and Silver Iminopyrrolidinates for}

Chemical Vapour Deposition of Metal Films

\section{Modified from original manuscript published as:}

Jason P. Coyle ${ }^{1}$, Peter G. Gordon ${ }^{1}$, Adam P. Wells ${ }^{1}$, David J. Mandia ${ }^{1}$, Eric R. Sirianni ${ }^{2}$, Glenn P. A. Yap ${ }^{2}$, Seán T. Barry “"Thermally Robust Gold and Silver Iminopyrrolidinates for Chemical Vapor Deposition of Metal Films" Chem. Mater. 2013, $25,4566-457$

${ }^{1}$ Department of Chemistry, Carleton University, 1125 Colonel By Drive, Ottawa, K1S 5B6, Canada

${ }^{2}$ Department of Chemistry \& Biochemistry, University of Delaware, Newark, Delaware 19716, United States

\subsection{Abstract}

Dimeric silver(I) and gold(I) tert-butyl-imino-2,2-dimethylpyrrolidinates were synthesised and characterised by thermal gravimetric analysis (TGA), differential scanning calorimetry (DSC), single crystal X-ray diffraction and chemical vapour deposition (CVD) experiments. The motivation for this work arose from the excellent thermal stability of the previously reported analogous copper(I) compound and to complete a series of potential precursors for atomic layer deposition. These compounds are stable in air and soluble in aromatic or chlorinated solvents. In the solid state, TGA and DSC showed these compounds to be thermally stable up to $170{ }^{\circ} \mathrm{C}$ and to have good evaporation yields up to $240{ }^{\circ} \mathrm{C}$. Gas phase decomposition temperatures were $140{ }^{\circ} \mathrm{C}$ for the silver compound and $300{ }^{\circ} \mathrm{C}$ for the gold compound, as determined by CVD experiments, where these compounds decomposed to produce metallic films. Reduction of the metal ions via dehydrogenation of the ligands is proposed as a thermolysis pathway.

\subsection{Introduction}


The preparation of metallic films of $\mathrm{Cu}, \mathrm{Ag}$, and $\mathrm{Au}$ have been a research interest for several decades. The coinage metals possess the lowest resistivities of all elements that are suitable for the interconnects in microelectronics. The surface plasmons of these noble metals ${ }^{1}$ are well known and they have been employed in photonic devices. ${ }^{2}$ Device fabrication, either for microelectronics or plasmonic devices, routinely employs chemical vapour deposition $^{3}$ (CVD) and atomic layer deposition ${ }^{4}$ (ALD) for thin film deposition as they are ideal technologies for coating geometrically complex substrates with high aspect ratio features in a wide variety of materials. ${ }^{5}$ The applicability of a CVD or ALD process relies heavily on its precursor chemistry which often has an important thermal component.

Precursors for CVD and ALD differ in their surface chemistry. A CVD precursor is thermally activated by a surface to form the target film either by decomposition or by reaction with a secondary gas. An ALD precursor is thermally activated to chemisorb to a surface forming a self-limiting monolayer that is stable throughout the subsequent purging steps until it reacts with a secondary precursor or reactant. The self-limiting monolayer consists of a surface species which is often poorly understood yet nevertheless prevents continuous uptake of gaseous precursor. Careful surface studies by sensitive techniques are required to fully understand the surface species' identity. ${ }^{6}$ The "ALD window" is a temperature range where the self-limiting monolayer exists and film growth can occur by reaction with a secondary precursor. This window is observed between the volatilisation temperature and decomposition temperature of the precursor. Generally, a precursor is limited for use in CVD when these two temperatures are not adequately separated. 
For copper thin films, the list of precursors for ALD and CVD include many examples of $\mathrm{Cu}(\mathrm{I})$ and $\mathrm{Cu}(\mathrm{II})$ homoleptic compounds including $\beta$-diketonates, ${ }^{7} \beta$ diketiminates, ${ }^{8}$ amidinates, ${ }^{9}$ guanidinates, ${ }^{10}$ aminoalkoxides ${ }^{11}$ and pyrrolylaldiminates. ${ }^{12}$ Interestingly, the list of homoleptic precursors for silver and gold is not as populated. This can be largely attributed to the larger ionic radii of silver and gold compared to copper. Typically, coinage metal precursors are found in the +1 oxidation state where copper commonly forms dimeric compounds with the ligands listed above. For silver(I) and gold(I), non-volatile oligomers or polymers are obtained which require stabilising Lewis bases to form monomeric or lower-order oligomeric volatile compounds. ${ }^{13}$ There exists a larger library of copper precursors due to an industry driven search for the optimal precursor for the future of interconnects in microchip fabrication. ${ }^{14}$ Identifying precursors for silver and gold thin films have generally been an academic pursuit with potential industrial application. Recently, a previously known CVD precursor for silver films has been reinvestigated and employed as a plasma enhanced-ALD precursor ${ }^{15}$ for the coating of high-aspect ratio substrates for surface-enhanced Raman spectroscopy (SERS) applications. ${ }^{16}$ A gold guanidinate ${ }^{17}$ has been used to coat fibre optics to build plasmonic devices. ${ }^{18}$

From the literature, there exist only a few examples of a ligand for which a complete analogous series of copper, silver, and gold compounds can be prepared for comparison. For example, homoleptic coinage metal hexamethyldisilazide compounds form exclusively tetramers. ${ }^{19}$ These compounds are thermally robust with high melting points; however, only $\left[\mathrm{CuN}\left(\mathrm{SiMe}_{3}\right)_{2}\right]_{4}$ has been used as a CVD precursor. ${ }^{19 \mathrm{a}}$ Coinage metal N, N' dialkylamidinates favour dimeric structures with one example of a silver 
amidinate existing as a dimer/trimer mixture in solution and the solid state. ${ }^{9 \mathrm{~d}}$ Copper amidinates are well-known precursors for $\mathrm{ALD}^{9 \mathrm{a}}$ but silver and gold amidinates volatilize and decompose within the same temperature range. Coinage metal alkyl substituted guanidinates (Figure 5.1) have shown very similar behavior to these alkyl amidinates and have been used as CVD precursors in homebuilt reactors. ${ }^{10,17}$ Similarly, coinage metal carboxylates and perfluorinated carboxylates show a similar trend within this chemical group $^{20}$ - generally, the copper compounds are volatile and useful as precursors for CVD whereas the silver and gold analogues suffer from low thermal stability or low volatility which impacts their utility as precursors.
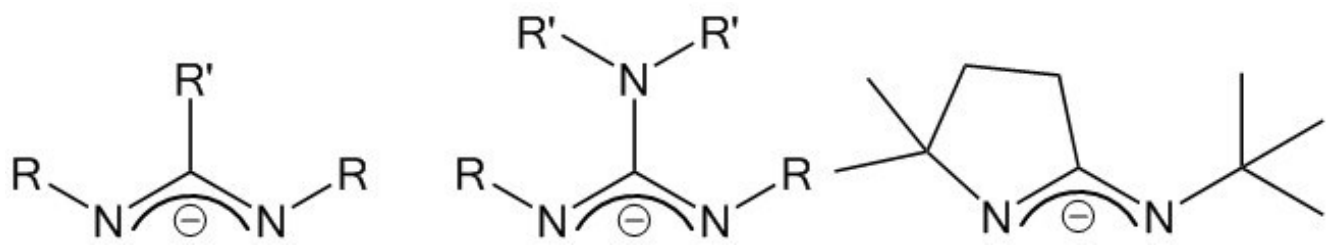

Figure 5.1 Amidinates, Guanidinates, and Iminopyrrolidinates Used as Ligands For Precursors in CVD and ALD (R, R' = any alkyl group).

Since it is rare for one ligand system to work well across group 11, precursor design has been developed individually for copper, silver and gold heteroleptic ligand systems. In addition to the library of homoleptic copper precursors mentioned above, there are additional entries from monomeric copper(I) compounds stabilised by Lewis bases. CupraSelect ${ }^{\mathrm{TM}}$, (hfac)Cu(TMVS), is a well-known copper precursor for $\mathrm{CVD}^{21}$ tethering of the vinyl silane Lewis base to a $\beta$-ketoiminate ligand increases thermal stability. ${ }^{22}$ Successful silver precursors have been synthesised using oxygen based ligands (carboxylates, $\beta$-diketonates) stabilized by phosphines, ${ }^{13 a}$ alkenes,${ }^{23}$ alkynes,${ }^{24}$ or isocyanides. ${ }^{25}$ Gold precursors can be achieved by ligand systems analogous to silver precursors ${ }^{26}$ or by exploiting the stability of methyl $\mathrm{Au}(\mathrm{I})^{27}$ and $\mathrm{Au}(\mathrm{III})^{28}$ compounds. A 
variety of $\mathrm{Me}_{2} \mathrm{Au}(\mathrm{III}) \mathrm{L}$ compounds have been tested as precursors (where $\mathrm{L}=\beta$ diketonates, carboxylates, thiocarbamates, salicylaldiminates, and dialkyldithiophosphates).

Recently, our group has studied the thermolysis mechanisms of a copper(I) amidinate $^{29}$ and a guanidinate ${ }^{30}$ in the gas phase. These compounds can undergo $\beta$ hydride elimination during decomposition producing metallic films. Redesigning the amidinate framework to replace $\beta$-hydrogen atoms with methyl groups affords an exceptionally stable, volatile copper compound. ${ }^{31}$ This redesign also incorporated a five-

membered ring which would prevent carbodiimide deinsertion ${ }^{30,32}$ and it also lessened the steric bulk of the ligand to facilitate the formation of a dimer. Herein, we present the analogous silver(I) and gold(I) compounds and report on their thermal stability, preliminary results from CVD, and considerations as potential ALD precursors.

\subsection{Results and Discussion}

Compounds $\mathbf{1}$ and $\mathbf{2}$ were generated by salt metathesis of lithium tert-butyl-imino2,2-dimethylpyrrolidinate and a metal chloride salt in fair yields (Figure 5.2). The isolated compounds were air stable and their solubility was limited to benzene, toluene or methylene chloride. These compounds were insoluble in saturated, aliphatic solvents, and generally unstable in polar solvents with the exception of methylene chloride. The isolated yields were lower than other silver and gold amidinates ${ }^{17}$ and this difference was attributed to difficulties extracting the product from the crude mixture. The ${ }^{1} \mathrm{H}$ NMR spectrum of $\mathbf{1}$ and $\mathbf{2}$ in $\mathrm{C}_{6} \mathrm{D}_{6}$ was simple to interpret and consistent with the expectation for a single chemical environment. Compound $\mathbf{2}$ could also be measured in $\mathrm{CDCl}_{3}$ and $\mathbf{1}$ decomposed immediately in $\mathrm{CDCl}_{3}$. 


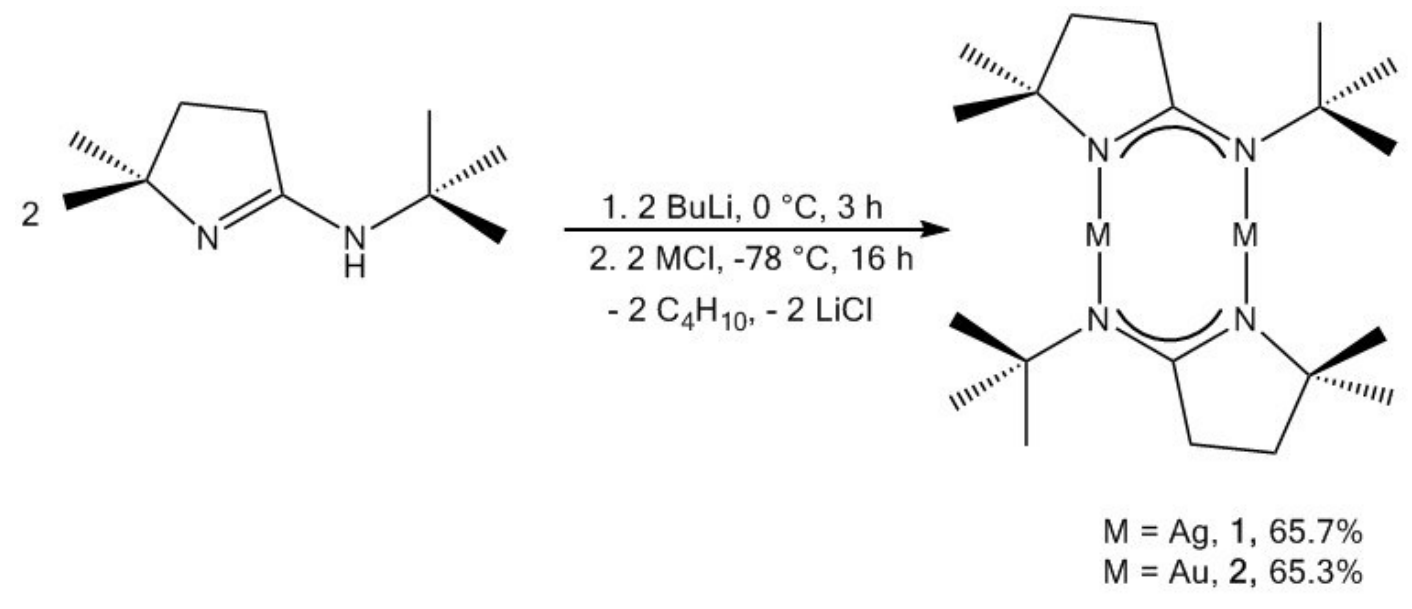

Figure 5.2 Synthesis of Silver(I) and Gold(I) tert-butyl-imino-2,2-dimethylpyrrolidinate.

Compounds 1 and $\mathbf{2}$ share an isomorphic dimeric structure with the previously reported analogous copper compound ${ }^{31}(3)$ in the monoclinic $\mathrm{P} 2{ }_{1} / \mathrm{c}$ space group with two molecules in the unit cell each located on inversion centres (Figure 5.3, Table 5.1 \& 5.2). The metal-metal distances are 2.677(3) and 2.6801(4) A for Ag and Au, respectively, which is similar to previously reported dimeric amidinates. ${ }^{9 \mathrm{~d}, 17}$ In both structures, the metal ions deviate from the planar metallocycle core bending the $\mathrm{N}-\mathrm{M}-\mathrm{N}$ ideally linear geometry by $\sim 9^{\circ}$. The core $\mathrm{C}-\mathrm{N}$ bonds range from $1.32 \AA-1.34 \AA$ showing the expected delocalized bond character within the iminopyrrolidinate. The parallelogram formed by the four nitrogen atoms in the dimeric molecular core deviates from an ideal rectangle by $4.9(2)^{\circ}$ for 1 and $3.8(2)^{\circ}$ for $\mathbf{2}$ to accommodate for the slight differences in steric bulk at each end of the asymmetric ligand. 


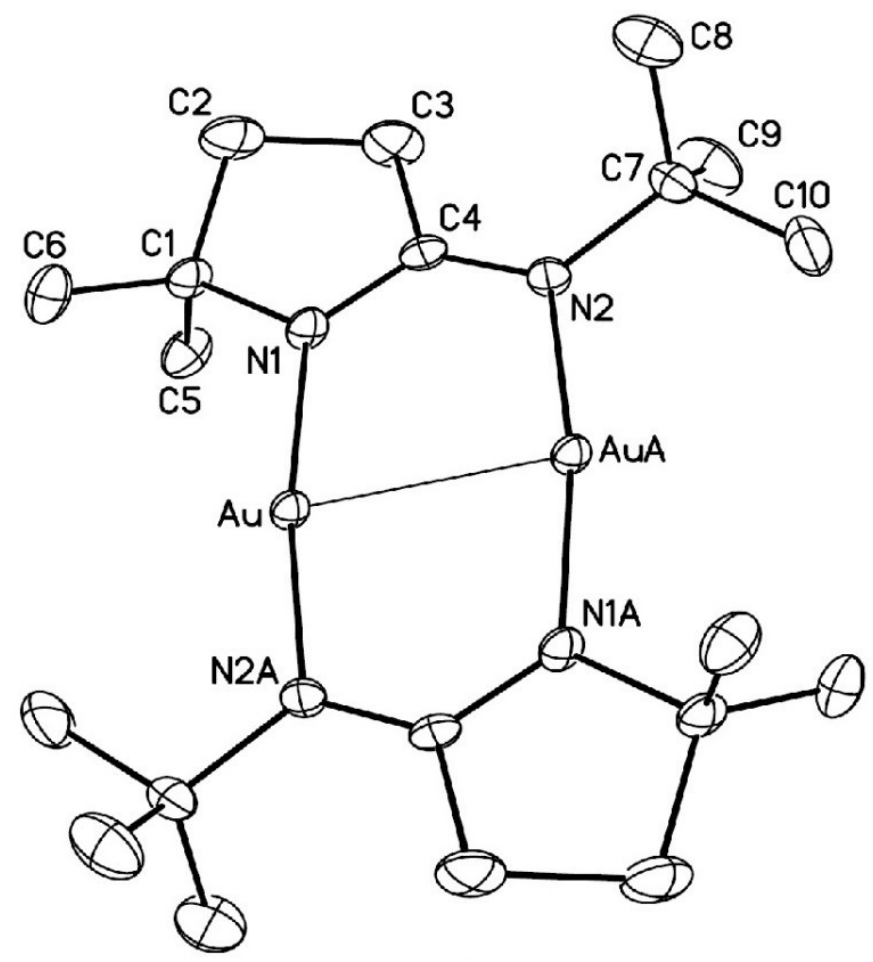

Figure 5.3 Molecular diagram of 2 with thermal ellipsoids at 30\% probability. H-atoms omitted for clarity. Ag analogue compound $\mathbf{1}$ is isomorphic with 2.

Table 5.1 Crystal Data and Refinement Details of 1 and 2.

\begin{tabular}{lll}
\hline & \multicolumn{1}{c}{1} & \multicolumn{1}{c}{$\mathbf{2}$} \\
\hline formula & $\mathrm{C}_{20} \mathrm{H}_{38} \mathrm{Ag}_{2} \mathrm{~N}_{4}$ & $\mathrm{C}_{20} \mathrm{H}_{38} \mathrm{Au}_{2} \mathrm{~N}_{4}$ \\
crystal system & monoclinic & monoclinic \\
space group & $P 2{ }_{1} / c$ & $P 2{ }_{1} / c$ \\
$a(\AA)$ & $10.124(10)$ & $10.1692(14)$ \\
$b(\AA)$ & $9.681(9)$ & $9.7129(13)$ \\
$c(\AA)$ & $12.008(12)$ & $12.0353(17)$ \\
$\beta\left({ }^{\circ}\right)$ & $106.047(13)$ & $106.605(2)$ \\
$V\left(\AA^{3}\right)$ & $1131.0(19)$ & $1139.2(3)$ \\
$Z$ & 2 & 2 \\
$\rho\left(\mathrm{g} / \mathrm{cm}^{3}\right)$ & 1.616 & 2.124 \\
$\mathrm{goodness}$ of fit & 1.082 & 1.039 \\
$\mathrm{R}\left(\mathrm{F}_{\mathrm{o}}\right)$ & 0.0235 & 0.0141 \\
$\mathrm{wR}\left(\mathrm{F}_{\mathrm{o}}\right)$ & 0.0564 & 0.0335 \\
\hline
\end{tabular}


Table 5.2 Selected Bond lengths and Angles for $\mathbf{1}$ and $\mathbf{2}$

\begin{tabular}{lclc}
\hline \multicolumn{2}{c}{$\mathbf{}$} & $\mathbf{2}$ \\
\hline selected bond & length $(\AA)$ & selected bond & length $(\AA)$ \\
\hline $\mathrm{Ag}-\mathrm{Ag}$ & $2.677(3)$ & $\mathrm{Au}-\mathrm{Au}$ & $2.6801(4)$ \\
$\mathrm{Ag}-\mathrm{N} 1$ & $2.085(2)$ & $\mathrm{Au}-\mathrm{N} 1$ & $2.026(2)$ \\
$\mathrm{Ag}-\mathrm{N} 2$ & $2.102(2)$ & $\mathrm{Au}-\mathrm{N} 2$ & $2.047(2)$ \\
$\mathrm{N} 1-\mathrm{C} 4$ & $1.324(3)$ & $\mathrm{N} 1-\mathrm{C} 4$ & $1.337(3)$ \\
$\mathrm{N} 2-\mathrm{C} 4$ & $1.323(3)$ & $\mathrm{N} 2-\mathrm{C} 4$ & $1.333(3)$ \\
\hline selected angle & angle $(\mathrm{deg})$ & $\mathrm{selected}$ angle & angle $(\mathrm{deg})$ \\
\hline $\mathrm{N} 1-\mathrm{Ag}-\mathrm{N} 2$ & $170.43(7)$ & $\mathrm{N} 1-\mathrm{Au}-\mathrm{N} 2$ & $170.90(9)$ \\
$\mathrm{N} 1-\mathrm{C} 4-\mathrm{N} 2$ & $123.6(2)$ & $\mathrm{N} 1-\mathrm{C} 4-\mathrm{N} 2$ & $124.7(2)$ \\
\hline
\end{tabular}

TGA of $\mathbf{1}$ and $\mathbf{2}$ showed single-feature weight loss curves with residual masses lower than the amount of metal contained in the sample. Equal molar amounts of $\mathbf{1}$ and $\mathbf{2}$ were heated under identical conditions and compared (Figure 5.4). The onset of mass loss ( $0.5 \%$ weight loss) for $\mathbf{1}$ was $172{ }^{\circ} \mathrm{C}$ and $209^{\circ} \mathrm{C}$ for $\mathbf{2}$. The residual mass from $\mathbf{1}$ was 13 $\%$ which indicates that at least $67 \%$ by mass of the silver in the sample volatilized. Likewise, the residual mass from 2 was $40 \%$ which indicates at least $26 \%$ by mass of the gold volatilized. Both compounds decomposed to metal as confirmed by powder Xray diffraction $(\mathrm{P}-\mathrm{XRD}){ }^{33}$ 


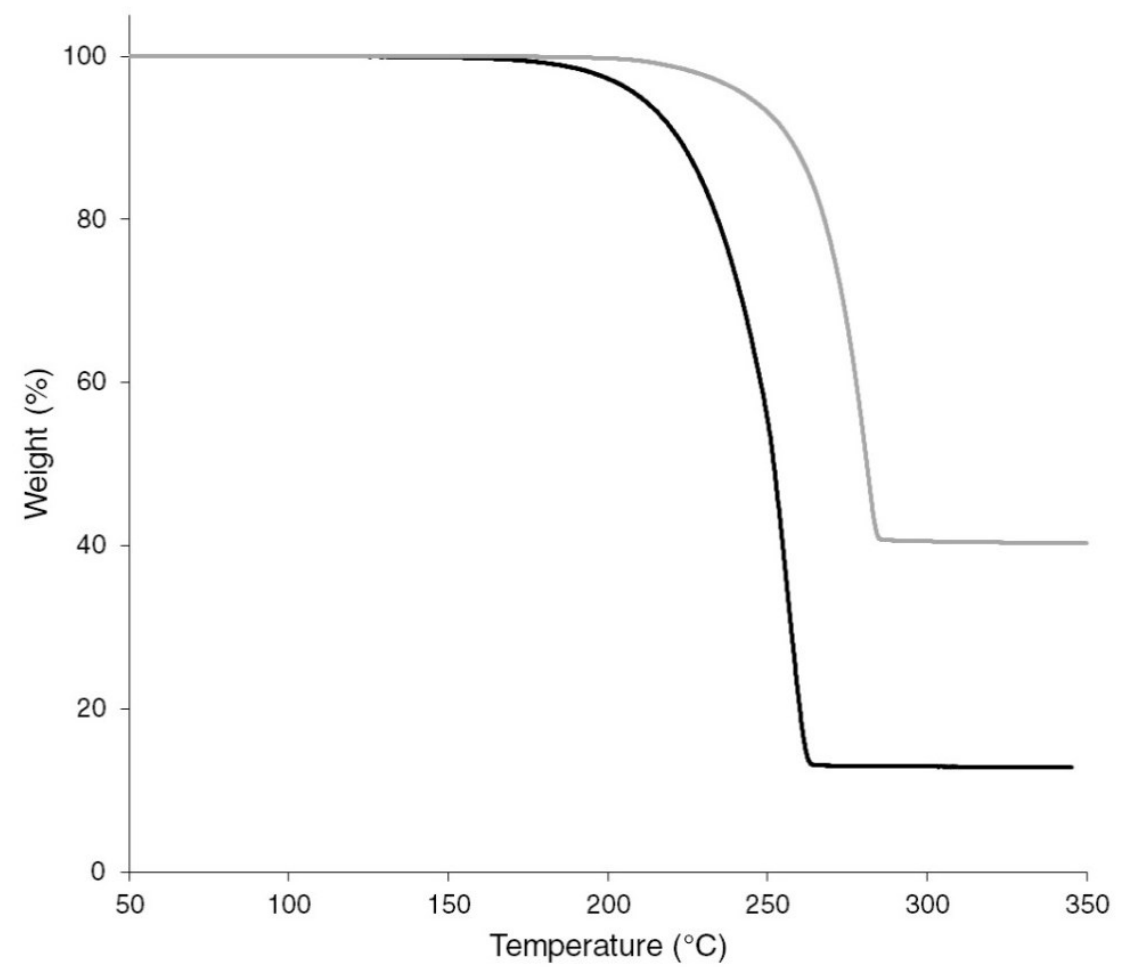

Figure 5.4 Thermogravimetric analysis of 1 (black) and $\mathbf{2}$ (gray). Experiments were run under $1 \mathrm{~atm}$ of nitrogen with a ramp rate of $10^{\circ} \mathrm{C} / \mathrm{min}$. Sample sizes for $\mathbf{1}$ and $\mathbf{2}$ were $10.49 \mathrm{mg}$ and $13.65 \mathrm{mg}$, respectively.

Isothermal data for 2 at $210^{\circ} \mathrm{C}, 230^{\circ} \mathrm{C}$, and $250^{\circ} \mathrm{C}$ (Figure 5.5 , b) yielded improved residual masses of $18 \%$ (at least $67 \%$ by mass of the gold volatilized). For $\mathbf{1}$, isothermal data at $180{ }^{\circ} \mathrm{C}, 210^{\circ} \mathrm{C}$, and $240{ }^{\circ} \mathrm{C}$ yielded residual masses of $7 \%, 6 \%$, and $17 \%$, respectively (Figure 5.5, a). The residual mass from $210^{\circ} \mathrm{C}$ shows an improved evaporation yield to $85 \%$ by mass of the silver contained in the sample whereas the isotherm at $240{ }^{\circ} \mathrm{C}$ shows increased decomposition than what was observed at $10{ }^{\circ} \mathrm{C} / \mathrm{min}$ (Figure 5.4). Comparison of the isothermal TGA of $\mathbf{1}$ and $\mathbf{2}$ highlights $\mathbf{2}$ as the more thermally robust compound. 

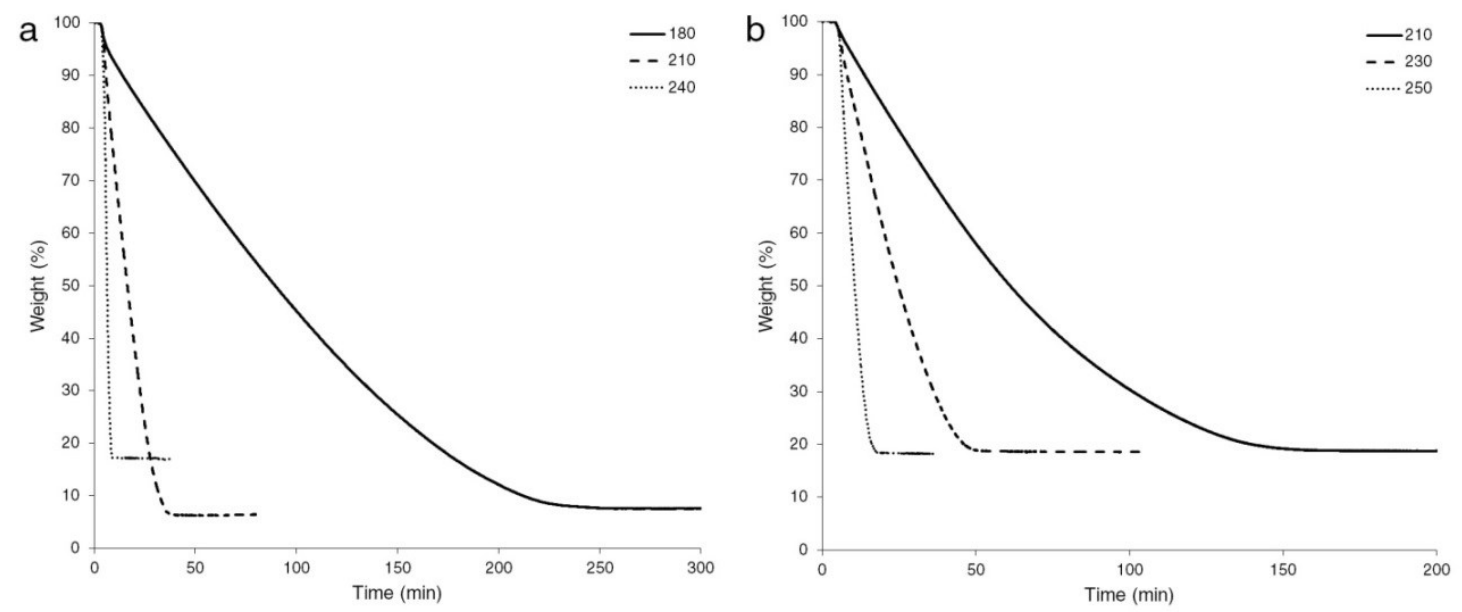

Figure 5.5 . Isothermal TGA of (a) 1 and (b) 2. The furnace was heated at $40{ }^{\circ} \mathrm{C} / \mathrm{min}$ up to the isothermal temperature. Isothermal temperatures are indicated in the right hand legend $\left({ }^{\circ} \mathrm{C}\right)$. Samples sizes were between $3.10 \mathrm{mg}$ to $3.67 \mathrm{mg}$.

DSC was performed to further evaluate the decomposition temperatures of $\mathbf{1}$ and 2 and compared to copper(I) tert-butyl-imino-2,2-dimethylpyrrolidinate (3) (Figure 5.6). DSC traces of 1 showed a broad exothermic peak between $180-253{ }^{\circ} \mathrm{C}$; whereas compound 2 showed a sharper decomposition event at $290{ }^{\circ} \mathrm{C}$. The DSC trace for 3 was strikingly different and showed endothermic events at $243{ }^{\circ} \mathrm{C}$ and $260{ }^{\circ} \mathrm{C}$ which were attributed to melting and boiling points, respectively. For $\mathbf{3}$, the endothermic events were repeatable once the sample pan was cooled to room temperature whereas the exothermic events for $\mathbf{1}$ and $\mathbf{2}$ were not repeatable thus indicative of decomposition. 


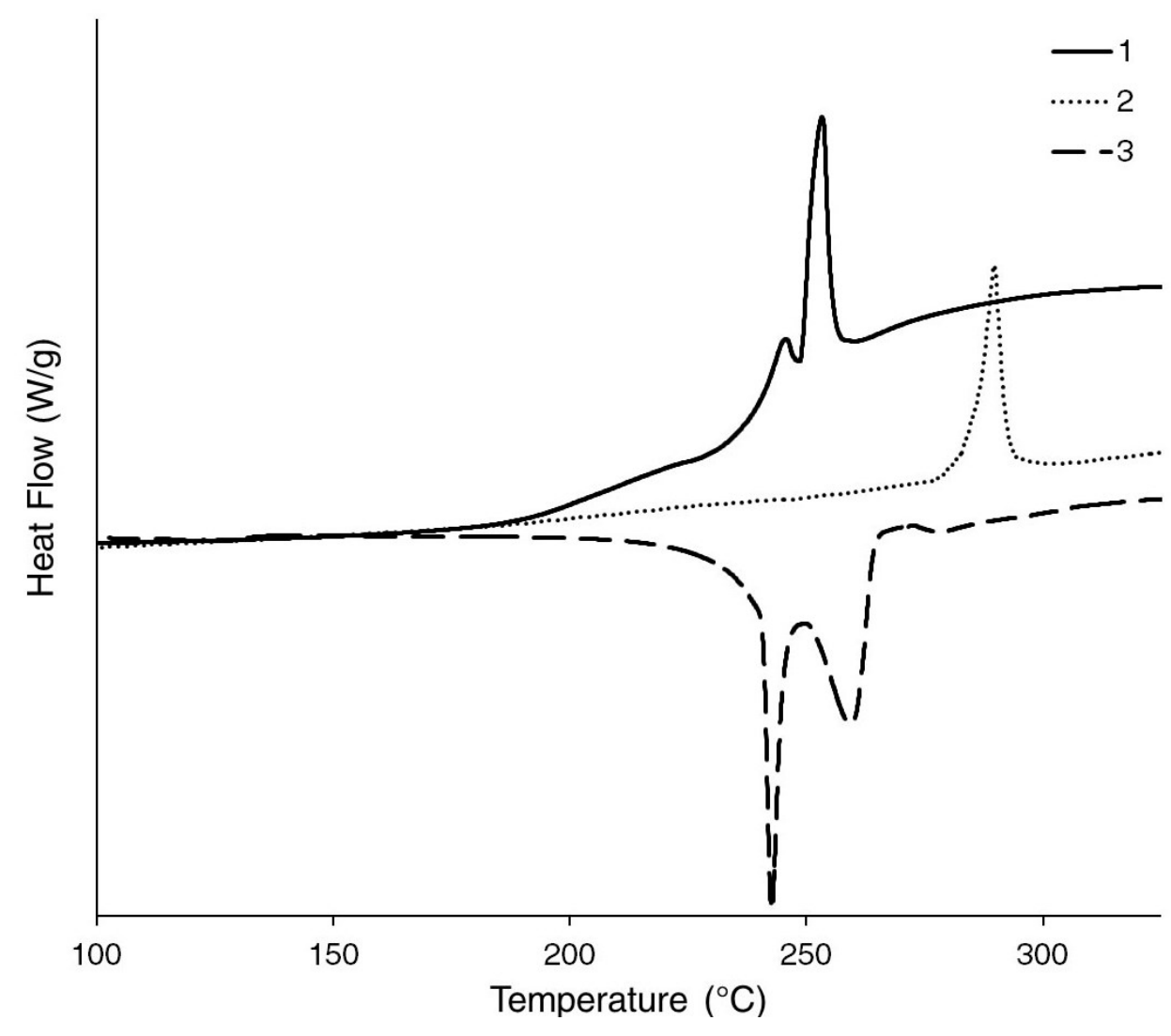

Figure 5.6 Differential scanning calorimetry of 1, 2, and $\mathbf{3}$ in hermetically sealed aluminum pans. Temperature ramp was $10{ }^{\circ} \mathrm{C} / \mathrm{min}$ up to $325^{\circ} \mathrm{C}$. Sample masses were $1.066 \mathrm{mg}, 1.625 \mathrm{mg}$, and $3.790 \mathrm{mg}$ for 1, 2, and 3, respectively. Exotherms are up.

The evaporation kinetics of $\mathbf{1}$ and $\mathbf{2}$ were evaluated by stepped isothermal TGA between $130{ }^{\circ} \mathrm{C}-190{ }^{\circ} \mathrm{C}$ (Figure 5.7) and compared to $3 .{ }^{34}$ Both compounds showed evaporation rates with Arrhenius temperature dependence and the expected trend for increasing molecular mass. Typically, iminopyrolidinates demonstrate decreased volatility when compared to acyclic amidinates and guanidinates largely due to their planar and rigid molecular structure. ${ }^{34}$ The design features of iminopyrrolidinates sufficiently increase thermal stability to allow for higher temperature volatility. From the stepped isothermal TGA and using the Langmuir equation, ${ }^{35}$ the temperature at which 1 Torr of vapor pressure could be obtained was estimated to be $222^{\circ} \mathrm{C}$ and $238^{\circ} \mathrm{C}$ for $\mathbf{1}$ and $\mathbf{2}$, respectively. 


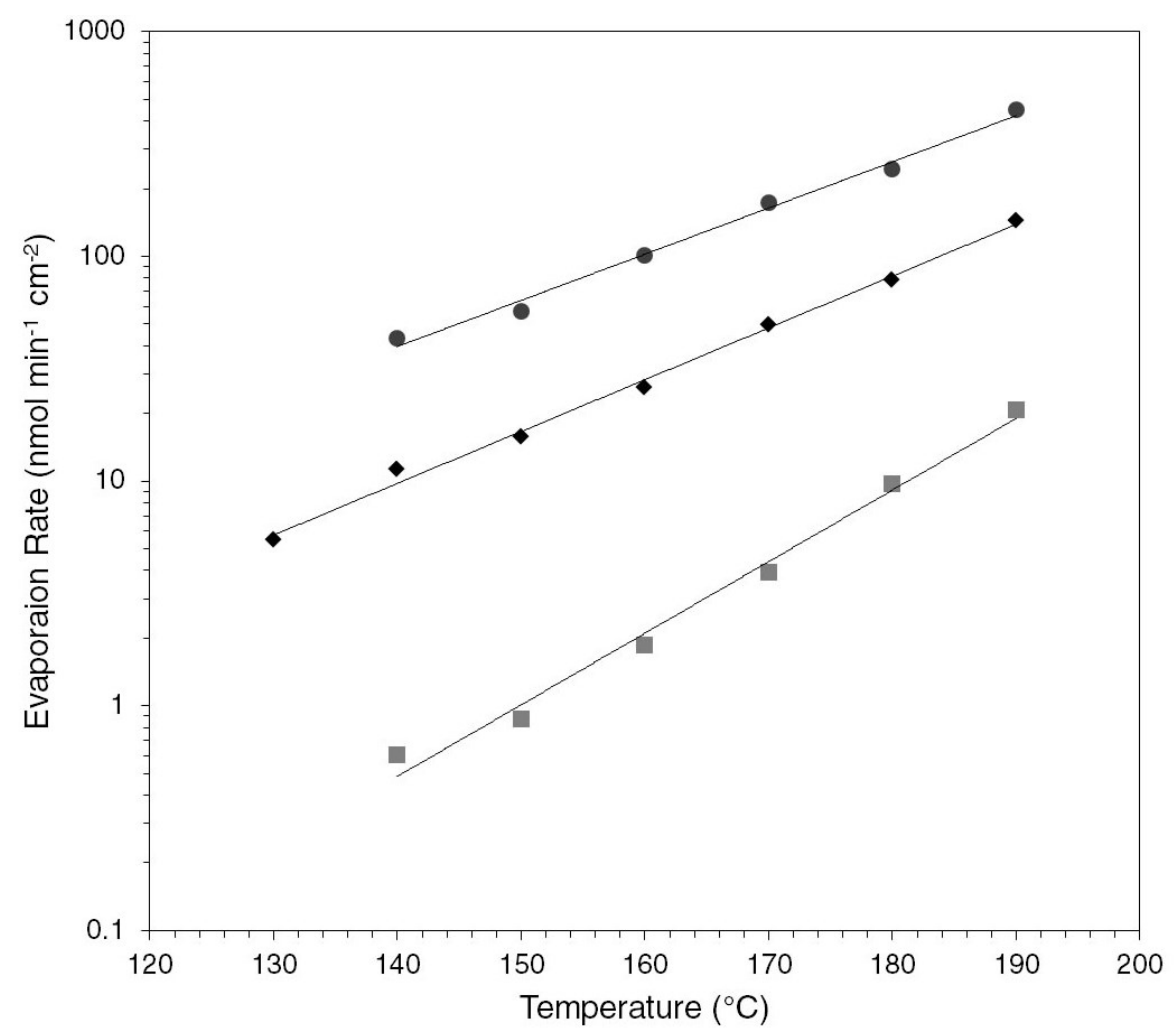

Figure 5.7 Evaporation kinetics of $\mathbf{1}(\bullet), 2(\square)$ and $\mathbf{3}(\bullet)$.

To further our understanding of the decomposition mechanism, solutions of $\mathbf{1}$ and 2 in $\mathrm{C}_{6} \mathrm{D}_{6}$ were sealed in heavy walled NMR tubes and heated in an isothermal oven equipped with a light bulb. Compound 2 decomposed at $100{ }^{\circ} \mathrm{C}$ whereas 1 required heating at $120^{\circ} \mathrm{C}$ to decompose within the same time frame as 2 (Figure 5.8). Solutions exposed to light completely decomposed in less time than solutions protected from light. Both compounds decomposed affording a metallic mirror plated on the NMR tube walls and protonated ligand as the only observable product by ${ }^{1} \mathrm{H}$ NMR. 


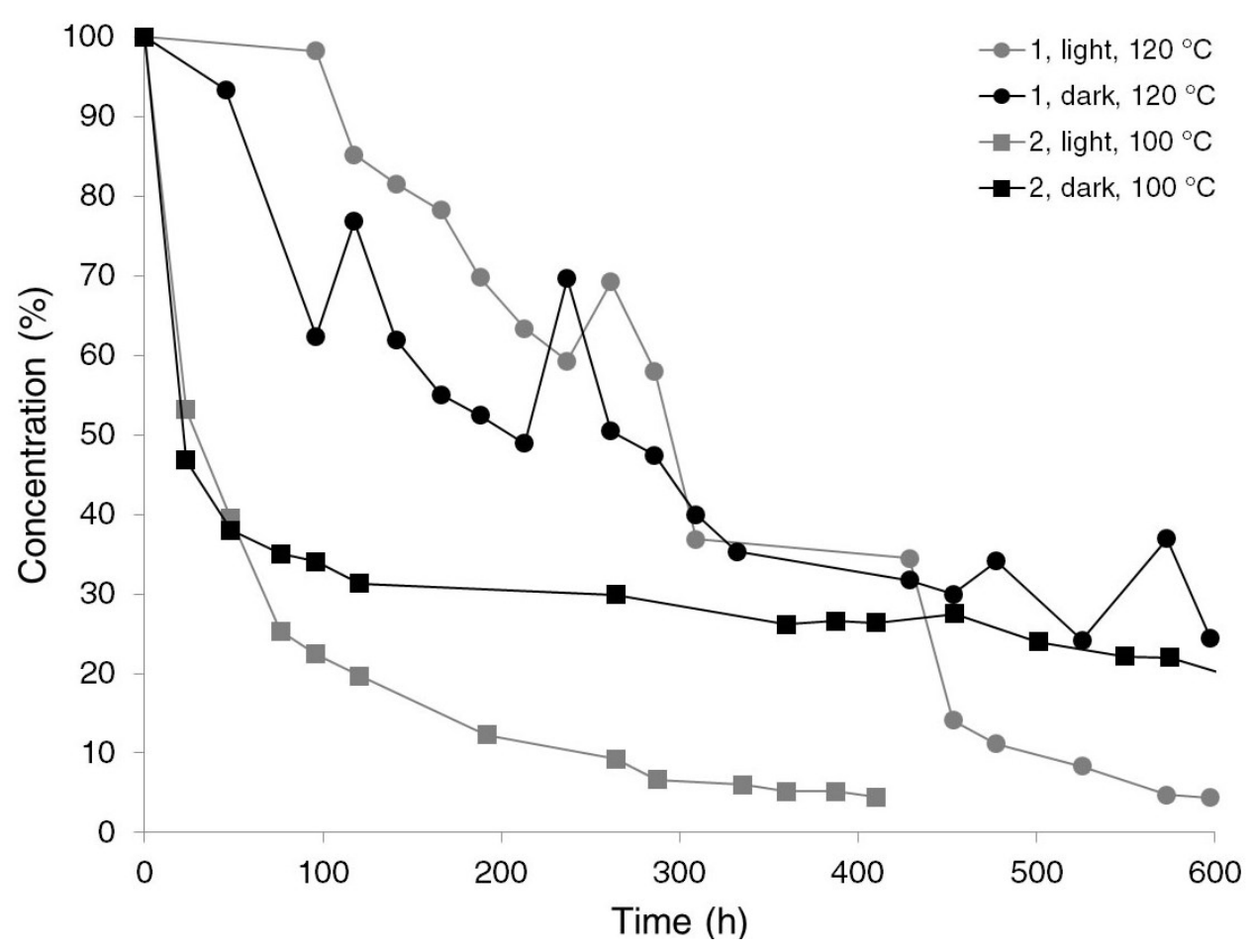

Figure 5.8 Decomposition of 1 and 2 in solution $\left(\mathrm{C}_{6} \mathrm{D}_{6}\right)$ measured by ${ }^{1} \mathrm{H}$ NMR.

The mechanism of decomposition remains unclear; however, these results are consistent with the thermolysis results of previously reported of copper(I) iminopyrrolidinates. ${ }^{34}$ Compounds $\mathbf{1}$ and $\mathbf{2}$ produced metallic residues during TGA and solution studies. Since the metal(I) ions were reduced, the ligand must have undergone sacrificial oxidation. Protonated ligand was the other mechanistic clue which suggests dehydrogenation of the sacrificial ligand. The calculated yields for protonated ligand in solution were between $30-80 \%$ of the initial concentration which suggests the unaccounted ligand could have been surface bound in a dehydrogenated state. Similar chemistry has been proposed for the surface decomposition of copper(I) amidinates, guanidinates, and iminopyrrolidinates. ${ }^{36}$

The promising results from thermolyses studies prompted preliminary investigations of $\mathbf{1}$ and $\mathbf{2}$ as precursors for CVD of metallic films. CVD experiments was 
carried out in a tube furnace reactor ${ }^{37}$ on $\mathrm{SiO}_{2}$ and $\mathrm{Si}$ substrates. The reactor was slightly modified and an external container was attached via a KF flange upstream on the tube furnace to allow for a separate heated zone for the precursor. For $\mathbf{1}$, the container was heated up to $110^{\circ} \mathrm{C}$ and maintained for $3 \mathrm{~h}$ at $35 \mathrm{mtorr}$. Approximately 50 to $60 \mathrm{mg}$ of 1

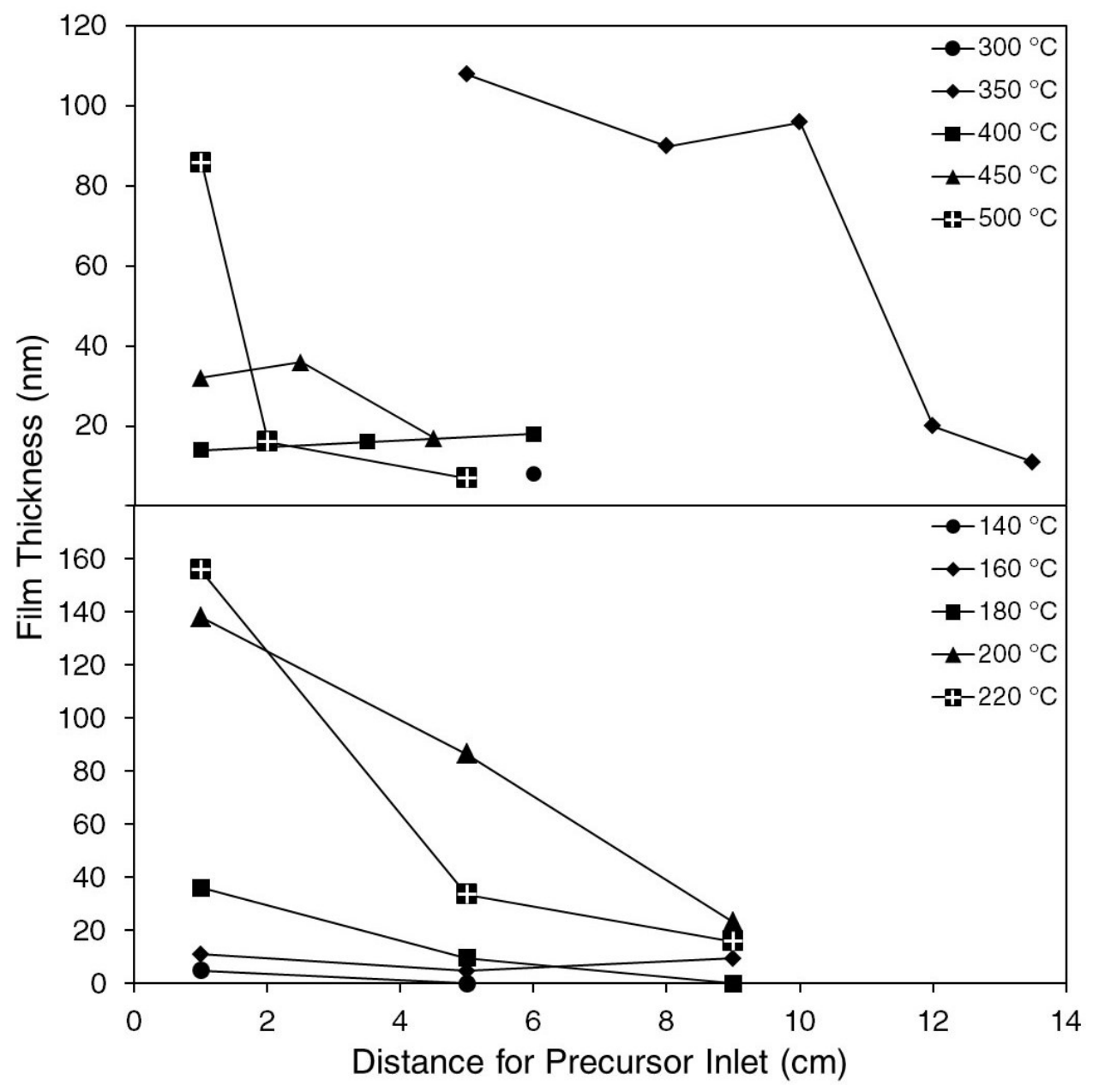

Figure 5.9 Film thickness determined from the CVD of 1 (bottom) and $\mathbf{2}$ (top) at different furnace temperatures. Depositions with 1 consumed $50 \mathrm{mg}-60 \mathrm{mg}$ of compound. Depositions from 2 consumed varying amounts: $13.9 \mathrm{mg}\left(300^{\circ} \mathrm{C}\right) ; 21.8 \mathrm{mg}$ $\left(350{ }^{\circ} \mathrm{C}\right) ; 5.9 \mathrm{mg}\left(400^{\circ} \mathrm{C}\right) ; 20.6 \mathrm{mg}\left(450^{\circ} \mathrm{C}\right) ; 23.5 \mathrm{mg}\left(500^{\circ} \mathrm{C}\right)$.

in an open glass vial was completely volatilized under these conditions for each deposition. The onset for film deposition was observed at $140{ }^{\circ} \mathrm{C}$ which afforded films less than $5 \mathrm{~nm}$ thick. Increasing the substrate temperature in $20^{\circ} \mathrm{C}$ increments from 140 
to $220^{\circ} \mathrm{C}$ afforded increasingly thicker films each with a thickness gradient receding from the precursor inlet (Figure 5.9, bottom). This series of depositions demonstrates 1 to be stable in the gas phase and at the substrate surface below $140{ }^{\circ} \mathrm{C}$ but is completely consumed in the tube furnace above temperatures of $200^{\circ} \mathrm{C}$. The deposited films were confirmed to be metallic silver by P-XRD.$^{33}$ Images obtained by scanning electron microscopy (SEM) showed the silver films deposited at $200{ }^{\circ} \mathrm{C}$ to be granular with particle sizes corresponding to film thickness (Figure 5.10; a, b).

Compound $\mathbf{2}$ was tested in a similar manner as a precursor for CVD of gold films on Si substrates. Approximately $30 \mathrm{mg}$ of 2 was heated to $130{ }^{\circ} \mathrm{C}$ over a $16 \mathrm{~h}$ deposition at 35 mtorr. Upon inspection of the precursor vial it was noticed that the mass of precursor was not entirely consumed; however, the material remaining consisted of completely intact precursor as determined by ${ }^{1} \mathrm{H}$ NMR analysis. The onset of film deposition was found to occur at $300{ }^{\circ} \mathrm{C}$. Increasing the tube furnace temperature in 50 ${ }^{\circ} \mathrm{C}$ increments from $300{ }^{\circ} \mathrm{C}$ to $500{ }^{\circ} \mathrm{C}$ afforded a thickness profile with a thickness maximum that migrated towards the precursor inlet at higher temperatures (Figure 5.9, top). The thickness profile observed for $\mathbf{2}$ was significantly different than $\mathbf{1}$ and we attribute this to a more pronounced temperature gradient across the tube furnace at higher temperatures. This series of depositions demonstrates $\mathbf{2}$ to be stable in the gas phase and at the substrate's surface below $300{ }^{\circ} \mathrm{C}$. Film thickness at the centre of the tube furnace reached a maximum at a temperature of $350{ }^{\circ} \mathrm{C}$ and deposition occurred predominately at the precursor inlet at a temperature of $500{ }^{\circ} \mathrm{C}$. The deposited films were confirmed to be metallic gold by P-XRD.$^{33}$ Images obtained by SEM of the gold films deposited at 350 ${ }^{\circ} \mathrm{C}$ consisted of densely packed particles that were $<50 \mathrm{~nm}$ (Figure $5.10 ; \mathrm{c}, \mathrm{d}$ ). 
Interestingly, film thickness did not correspond to particle size and the thicker areas appeared more continuous than granular. The films showed good adhesion by passing a Scotch tape test.

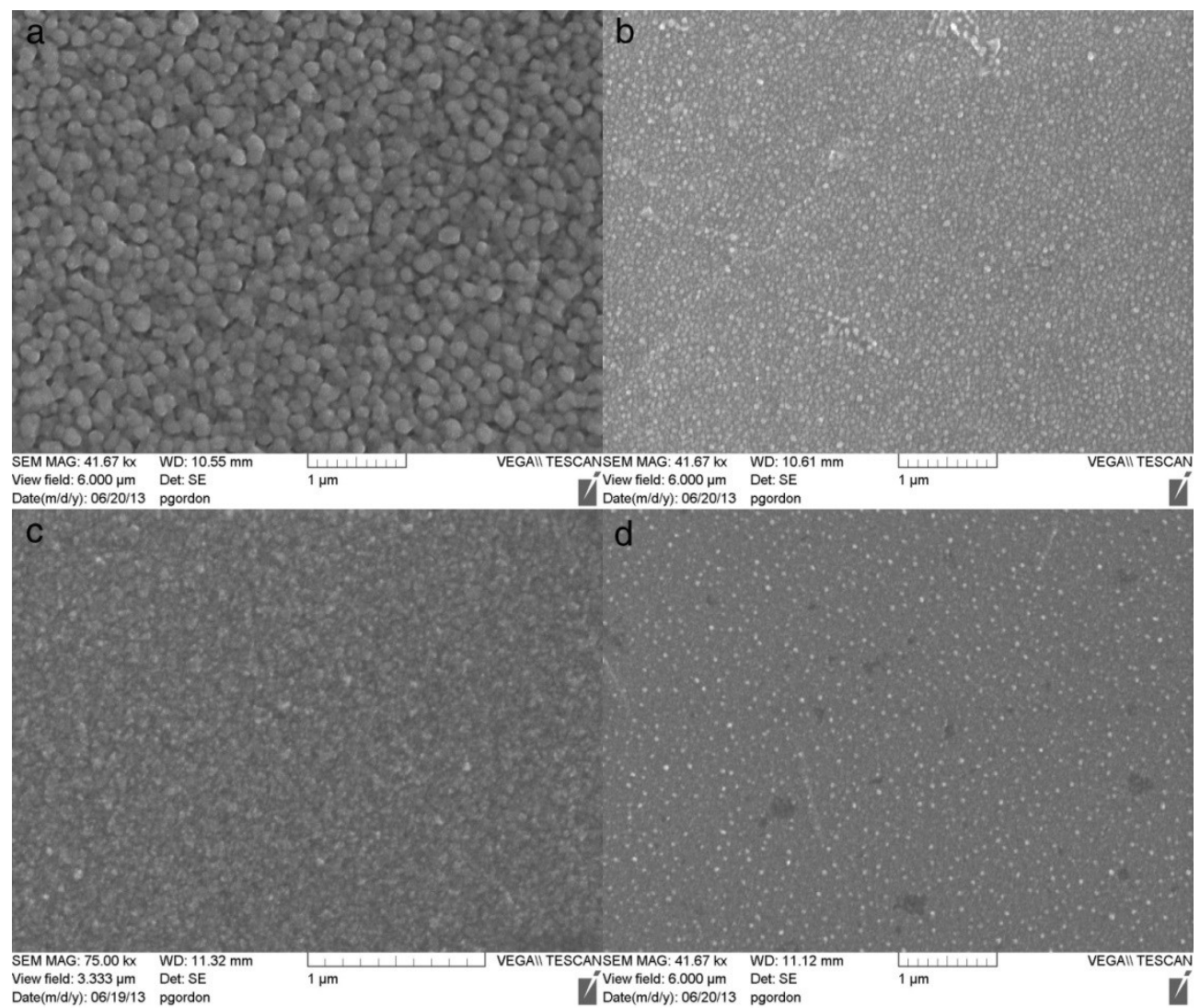

Figure 5.10 SEM images for thin films deposited from (a, b) 1 and (c, d) 2 at $200{ }^{\circ} \mathrm{C}$ and $350{ }^{\circ} \mathrm{C}$, respectively. Images from the CVD of $\mathbf{1}$ were measured (a) $0.75 \mathrm{~cm}$ and (b) 7.75 $\mathrm{cm}$ from the precursor inlet. Images from the CVD of 2 were measured (a) $5 \mathrm{~cm}$ and (b) $11 \mathrm{~cm}$ from the precursor inlet.

X-ray photoelectron spectroscopy (XPS) analysis after sputtering was performed to determine the bulk composition of the deposited films. The silver and gold films were of similar purity and contained only carbon as an impurity incorporated from the ligand system. A film deposited from $\mathbf{1}$ at $220{ }^{\circ} \mathrm{C}$ was determined to be 96.8 at. $\%$ silver and 2.7 at.\% carbon (Figure 5.12 in Supporting Information) and a film deposited from 2 at 350 ${ }^{\circ} \mathrm{C}$ was determined to be 96.8 at.\% gold and 3.2 at.\% carbon (Figure 5.13 in Supporting 
Information). The remaining composition of these films was oxygen $(<0.5$ at.\%) which either arose from exposure to air post-deposition or from background concentrations in the deposition system. Conductivity measurements of a $156 \mathrm{~nm}$ silver film deposited at $220{ }^{\circ} \mathrm{C}$ on a glass substrate gave a resistivity of $2.46 \mu \Omega \cdot \mathrm{cm}$ which is close to the value for bulk silver $(1.59 \mu \Omega \cdot \mathrm{cm})$. Conversely, a $96 \mathrm{~nm}$ gold film deposited at $350{ }^{\circ} \mathrm{C}$ on a silicon substrate gave a resistivity of $5.58 \mathrm{~m} \Omega \cdot \mathrm{cm}$ which is significantly higher than bulk gold $(2.44 \mu \Omega \cdot \mathrm{cm})$. The higher resistivity compared to bulk gold are probably not due to impurities but rather to poorly connected particles in the film.

In light of the film thickness gradient inherent to the crude reactor design, CVD experiments using a low flow of $\mathrm{N}_{2}$ gas were performed which successfully minimized thickness gradients across $20 \mathrm{~cm}$ of the furnace by increasing the rate of mass transport of 1 (Figure 5.11). The tube supplying $\mathrm{N}_{2}$ was heated prior to leaking $\mathrm{N}_{2}$ into the vacuum system behind the precursor. Negligible gradients were observed between $250{ }^{\circ} \mathrm{C}$ and $300{ }^{\circ} \mathrm{C}$; above $300{ }^{\circ} \mathrm{C}$ the rate of decomposition surpassed the rate of mass transport and a gradient was observed again. CVD with $\mathbf{1}$ under $\mathrm{N}_{2}$ flow required higher substrate temperatures either because the gas flow cooled the surfaces or because mass transport of the precursor drastically improved. Growth rates calculated from the data in Figure 5.11 gave values between $0.8 \mathrm{~nm} / \mathrm{min}$ and $1.1 \mathrm{~nm} / \min$ across $17 \mathrm{~cm}$ of the tube furnace $\left(\mathrm{R}^{2}\right.$ values of the linear fits for growth rate at each distance were between $0.87-0.98)$. A flow of $\mathrm{N}_{2}$ did not affect the film gradients or temperatures for CVD with $\mathbf{2}$. 


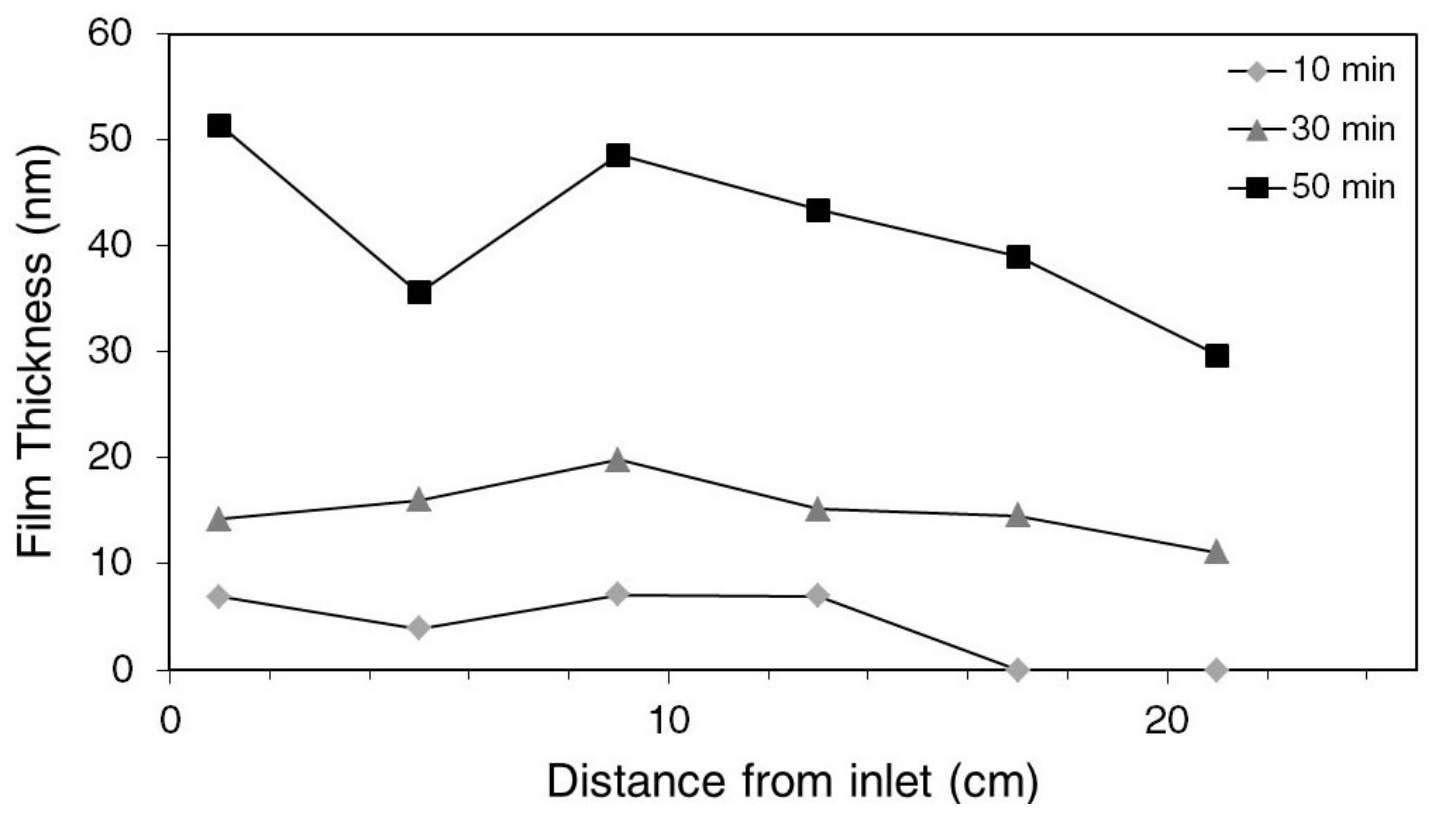

Figure 5.11 Film thickness determined from timed CVD experiments of $\mathbf{1}$ under a low flow of $\mathrm{N}_{2}$ at $300{ }^{\circ} \mathrm{C}$ with a system pressure of 100 mtorr.

These new compounds are significantly different than the commonly used precursors for the CVD of silver and gold mentioned above. The formulation of $\mathbf{1}$ and $\mathbf{2}$ as N-bonded, homoleptic dimers that are volatile and thermally robust is quite rare for silver and gold precursors. In consideration of volatility, $\mathbf{1}$ and $\mathbf{2}$ are less volatile than existing precursors; however, they do not require a fluorinated ligand or a Lewis base. These helpful strategies to increase volatility can lead to film impurities and precursor decomposition via dissociation of the Lewis base. Compound 1 deposits films at lower temperatures compared to previously reported precursors, which typically have minimum deposition temperatures $>200{ }^{\circ} \mathrm{C}$. On the other hand, $\mathbf{2}$ was found to require higher substrate temperatures than any previously reported gold precursor - a promising result for a potential ALD precursor. 


\subsection{Conclusion}

Two thermally robust CVD precursors for silver and gold thin films were synthesised and thermally characterized. Despite a lack of suitable solvents, the synthetic yields were satisfactory for our studies. Compounds $\mathbf{1}$ and $\mathbf{2}$ are thermally stable up to $170{ }^{\circ} \mathrm{C}$ as pure solids. Under low pressure CVD conditions, $\mathbf{1}$ and $\mathbf{2}$ could be volatilized without decomposition between $110{ }^{\circ} \mathrm{C}-130{ }^{\circ} \mathrm{C}$. Furthermore, these precursors remained predominately volatile up to $240{ }^{\circ} \mathrm{C}$ during atmospheric TGA.

TGA, DSC and CVD experiments determined $\mathbf{2}$ to be more thermally stable than 1. The series of coinage metal precursors had the following trend in thermal stability $\mathrm{Cu}$ $>\mathrm{Au}>\mathrm{Ag}$ in the solid or gaseous state. Solution based thermal studies revealed metallic mirrors and protonated ligand as the observable decomposition products. The order of thermal stability for $\mathbf{1}$ and $\mathbf{2}$ was reversed in $\mathrm{C}_{6} \mathrm{D}_{6}$ solutions and both compounds demonstrated enhanced decomposition when exposed to light.

Compounds $\mathbf{1}$ and $\mathbf{2}$ are improved precursors for CVD applications when compared to other amidinate and guanidinate compounds - their evaporation temperature and gas phase decomposition temperatures are well separated which is desirable for CVD and ALD. The employed ligand design was intended to block low temperature decomposition pathways of $\beta$-hydrogen abstraction and CDI de-insertion. ${ }^{30}$ Despite this attempt, $\mathbf{1}$ and $\mathbf{2}$ could still be thermally decomposed affording metallic residues and thin metal films - presumably by dehydrogenation of the ligand at higher temperatures. Metallic thin films of silver and gold consisting of nanoparticles were deposited between $140{ }^{\circ} \mathrm{C}-220{ }^{\circ} \mathrm{C}$ and $300{ }^{\circ} \mathrm{C}-500{ }^{\circ} \mathrm{C}$, respectively. Low film resistivities for silver films were achieved while the gold films gave resistivity values two orders of magnitude 
higher than bulk gold. This striking difference was likely due to differences in film morphology. Current investigations are focusing on optimizing CVD conditions and exploring reducing agents for ALD processes.

\subsection{Experimental}

General Considerations: Some manipulations (loading, filtering, and isolation) were performed in an MBraun Labmaster ${ }^{\mathrm{TM}} 130$ Dry box (mBraun, Stratham, NH, U.S.A.) under a nitrogen atmosphere while additions were performed by standard Schlenk techniques. NMR spectra were recorded on a $400 \mathrm{MHz}$ Bruker AMX spectrometer. NMR spectra were measured in $\mathrm{C}_{6} \mathrm{D}_{6}$ or $\mathrm{CDCl}_{3}$ and were referenced against residual protonated solvent or TMS, respectively. Tert-butyl-imino-2,2dimethylpyrrolidine ${ }^{31}$ and $\mathrm{THT} \cdot \mathrm{AuCl}^{38}$ were prepared according to literature. $\mathrm{AgCl}$ was purchased from Strem Chemicals and used as received. Toluene and diethyl ether were purchased as ACS grade and purified from an Mbraun Solvent Purifier System. Dichloromethane and pentane were purchased as anhydrous from Sigma Aldrich Chemical Company and used as received. Canadian Microanalytical Service Ltd. performed combustion analysis. Thermal gravimetric analysis was performed on a TA Instruments Q50 apparatus located in an MBraun Labmaster 130 Dry box under a nitrogen atmosphere. Differential scanning calorimetry was performed on a DSC Instruments Q10 apparatus.

Silver(I) tert-butyl-imino-2,2-dimethylpyrrolidinate (1): Tert-butyl-imino-2,2dimethylpyrrolidine $(3.364 \mathrm{~g}, 20.0 \mathrm{mmol})$ was dissolved in $60 \mathrm{~mL}$ of toluene and cooled to $0{ }^{\circ} \mathrm{C}$. Butyl lithium in hexanes $(2.5 \mathrm{M}, 8.0 \mathrm{~mL}, 20 \mathrm{mmol})$ was added dropwise and the solution was stirred for $3 \mathrm{~h}$ at room temperature. In a separate $500 \mathrm{~mL}$ Schlenk flask 
wrapped in aluminum foil, $\mathrm{AgCl}(2.865 \mathrm{~g}, 20.0 \mathrm{mmol})$ was suspended in $200 \mathrm{~mL}$ of toluene. The lithiated imino-pyrrolidinate solution was added dropwise via cannula over $1 \mathrm{~h}$ and the resulting mixture was stirred for $16 \mathrm{~h}$. The reaction mixture was filtered and the insoluble solids were washed with $20 \mathrm{~mL}$ of toluene. Volatiles were vacuum distilled from the combined washings and filtrate to afford a light brown solid. The insoluble solids were washed further with dichloromethane $(3 \times 20 \mathrm{~mL})$. These washings were combined and the volatiles were vacuum distilled to afford more of the light brown solid. The two portions of solid were combined and washed with pentane $(4 \times 10 \mathrm{~mL})$. The crude solid was recrystallized from $20 \mathrm{~mL}$ of hot toluene in a pressure vessel to afford colourless, block crystals; $3.615 \mathrm{~g}, 65.7 \% .{ }^{1} \mathrm{H}$ NMR $\left(400 \mathrm{MHz}, \mathrm{C}_{6} \mathrm{D}_{6}\right): \delta 1.16(\mathrm{~s}, 6 \mathrm{H}$, $\left.\mathrm{NC}\left(\mathrm{CH}_{3}\right)_{2} \mathrm{CH}_{2} \mathrm{CH}_{2} \mathrm{C}\right), \delta 1.29\left(\mathrm{~s}, 9 \mathrm{H}, \mathrm{NC}\left(\mathrm{CH}_{3}\right)_{3}, \delta 1.65\left(\mathrm{t}, 2 \mathrm{H}, \mathrm{NC}\left(\mathrm{CH}_{3}\right)_{2} \mathrm{CH}_{2} \mathrm{CH}_{2} \mathrm{C}\right), \delta\right.$ $2.54\left(\mathrm{t}, 2 \mathrm{H}, \mathrm{NC}\left(\mathrm{CH}_{3}\right)_{2} \mathrm{CH}_{2} \mathrm{CH}_{2} \mathrm{C}\right){ }^{13} \mathrm{C} \mathrm{NMR}\left(400 \mathrm{MHz}, \mathrm{C}_{6} \mathrm{D}_{6}\right): 32.9$ $\left(\mathrm{NC}\left(\mathrm{CH}_{3}\right)_{2} \mathrm{CH}_{2} \mathrm{CH}_{2} \mathrm{C}\right), 33.8\left(\mathrm{NC}\left(\mathrm{CH}_{3}\right)_{2} \mathrm{CH}_{2} \mathrm{CH}_{2} \mathrm{C}\right), 34.0\left(\mathrm{NC}\left(\mathrm{CH}_{3}\right)_{3}\right), 38.3$

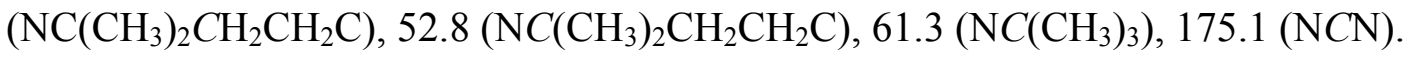
Combustion analysis, found(calculated): C, 43.21(43.65); H, 7.04(6.96); N, 10.15(10.18). Gold(I) tert-butyl-imino-2,2-dimethylpyrrolidinate (2): Tert-butyl-imino-2,2dimethylpyrrolidine (2.874 g, $17.1 \mathrm{mmol})$ was dissolved in $60 \mathrm{~mL}$ of toluene and cooled to $0{ }^{\circ} \mathrm{C}$. Butyl lithium in hexanes $(2.5 \mathrm{M}, 6.83 \mathrm{~mL}, 17 \mathrm{mmol})$ was added dropwise and the solution was stirred for $3 \mathrm{~h}$ at room temperature. In a separate $500 \mathrm{~mL}$ Schlenk flask wrapped in aluminum foil, THT $\mathrm{AuCl}(5.475 \mathrm{~g}, 17.1 \mathrm{mmol})$ was suspended in $175 \mathrm{~mL}$ of toluene and cooled to $-78^{\circ} \mathrm{C}$. The lithiated imino-pyrrolidinate solution was added dropwise via cannula over $1 \mathrm{~h}$ and the resulting mixture was stirred for $16 \mathrm{~h}$ while gradually warming to room temperature. Volatiles were vacuum distilled to afford a dark 
solid which was dried under high-vac to remove residual THT. The solid was stirred with warm toluene $(4 \times 50 \mathrm{~mL})$ for $15 \mathrm{~min}$ and filtered. The extractions were combined and volatiles were vacuum distilled to afford a solid that was washed with diethyl ether (4 $\mathrm{x}$ $5 \mathrm{~mL}$ ). The solid was dissolved in $20 \mathrm{~mL}$ of hot toluene in a pressure vessel and left to stand for $12 \mathrm{~h}$ cooling to room temperature. A crop of colourless, block crystals were isolated by decanting the supernatant, washing with diethyl ether $(3 \times 5 \mathrm{~mL})$ and dried under vacuum to afford $2.100 \mathrm{~g}$. A second crop of product was obtained from the insoluble solid that remained post toluene extraction. The remaining insoluble solids were stirred with dichloromethane $(4 \times 50 \mathrm{~mL})$ for $15 \mathrm{~min}$ and filtered. The extractions were combined and volatiles were vacuum distilled to afford a solid that was washed with diethyl ether $(4 \times 5 \mathrm{~mL})$. The solid was dissolved in $20 \mathrm{~mL}$ of hot toluene in a pressure vessel and left to stand for $12 \mathrm{~h}$ cooling to room temperature. A second crop of colourless, block crystals were isolated by decanting the supernatant, washing with diethyl ether $(3 \times 5 \mathrm{~mL})$ and drying under vacuum to afford $1.960 \mathrm{~g}$; total yield was 4.060 g, 65.3\%. ${ }^{1} \mathrm{H}$ NMR (400 MHz, $\left.\mathrm{CDCl}_{3}\right): \delta 1.18\left(\mathrm{~s}, 6 \mathrm{H}, \mathrm{NC}\left(\mathrm{CH}_{3}\right)_{2} \mathrm{CH}_{2} \mathrm{CH}_{2} \mathrm{C}\right), \delta 1.38$ (s, $\left.9 \mathrm{H}, \mathrm{NC}\left(\mathrm{CH}_{3}\right)_{3}\right), \delta 1.72\left(\mathrm{t}, 2 \mathrm{H}, \mathrm{NC}\left(\mathrm{CH}_{3}\right)_{2} \mathrm{CH}_{2} \mathrm{CH}_{2} \mathrm{C}\right), \delta 2.73\left(\mathrm{t}, 2 \mathrm{H}, \mathrm{NC}\left(\mathrm{CH}_{3}\right)_{2} \mathrm{CH}_{2} \mathrm{CH}_{2} \mathrm{C}\right)$ ${ }^{13} \mathrm{C}$ NMR $\left(400 \mathrm{MHz}, \mathrm{CDCl}_{3}\right): \delta 30.5\left(\mathrm{NC}\left(\mathrm{CH}_{3}\right)_{2} \mathrm{CH}_{2} \mathrm{CH}_{2} \mathrm{C}\right), \delta 33.7\left(\mathrm{NC}\left(\mathrm{CH}_{3}\right)_{3}\right), \delta 34.2$ $\left(\mathrm{NC}\left(\mathrm{CH}_{3}\right)_{2} \mathrm{CH}_{2} \mathrm{CH} \mathrm{H}_{2} \mathrm{C}\right), \delta 36.8\left(\mathrm{NC}\left(\mathrm{CH}_{3}\right)_{2} \mathrm{CH}_{2} \mathrm{CH}_{2} \mathrm{C}\right), \delta 56.0\left(\mathrm{NC}\left(\mathrm{CH}_{3}\right)_{2} \mathrm{CH}_{2} \mathrm{CH}_{2} \mathrm{C}\right), \delta$ $63.4\left(\mathrm{NC}\left(\mathrm{CH}_{3}\right)_{3}\right), \delta 176.5(\mathrm{NCN})$ Combustion analysis, found(calculated): $\mathrm{C}$, 32.85(32.98); H, 5.23(5.26); N, 7.67(7.69).

Crystallography Crystal Structure studies for compounds 1 and 2. Crystals were selected, sectioned as required, and mounted on plastic mesh with viscous oil and flashcooled to the data collection temperature. Diffraction data were collected on a Bruker- 
AXS APEX II Duo CCD diffractometer with graphite-monochromated Mo-Ka radiation $(\lambda=0.71073 \AA)$. The data-sets were treated with SADABS absorption corrections based on redundant multiscan data. The unit cell parameters and systematic absences in the diffraction data were uniquely consistent for $P 2_{1} / c$. The isomorphic structures were solved using direct methods and refined with full-matrix, least-squares procedures on $F^{2}$. Unit cell parameters were determined by sampling three different sections of the Ewald sphere. Nonhydrogen atoms were refined with anisotropic displacement parameters. Hydrogen atoms were treated as idealized contributions. Structure factors and anomalous dispersion coefficients are contained in the SHELXTL 6.12 program library. ${ }^{39}$ The structures have been deposited at the Cambridge Crystallographic Data Centre under CCDC $949353 \& 949354$

Chemical Vapor Deposition: All depositions were performed in a home built low pressure hot-walled CVD apparatus. ${ }^{37} \mathrm{Si}\left(\begin{array}{lll}1 & 0 & 0\end{array}\right)$ wafers and $\mathrm{SiO}_{2}$ soda-lime glass microscope slides were cleaned with piranha solution $\left(\mathrm{H}_{2} \mathrm{SO}_{4}\right.$ and $\mathrm{H}_{2} \mathrm{O}_{2}$ in a 3:1 mixture) for 20 minutes and air dried after rinsing with copious amounts of distilled water. Substrates were allowed to equilibrate in a removable stainless steel boat at each furnace temperature for 30 minutes under high vacuum. The external precursor container was heated independently with resistive heating tape. Timing for each deposition experiment began once the precursor container reached the setpoint temperature and finished when 1 atm of $\mathrm{N}_{2}$ was admitted to the apparatus. The substrates were removed once cooled.

Film Characterization: Scanning electron microscopy (SEM, TESCAN) and atomic force microscopy (AFM, Nanosurf) were used to study the surface morphology and film thicknesses. The AFM software analysis package WSxM 5.0 was used for film 
thickness and roughness calculations. ${ }^{40}$ To determine film thickness the film was first scratched with a knife and the step height was measured by AFM. A histogram (count of feature heights) was extracted from the AFM data for a field of view that included roughly equal areas of film and substrate along the scratch. The difference between the two histogram maxima was then taken as the thickness of the film. Film thickness values corroborated with values determined from SEM interrogation of cross-sections. Sheet resistance was measured with a four-point probe. The elemental composition was determined by XPS using a custom-built, ultra-high vacuum SPECS (GmbH) XPS system (see Supporting Information). XPS spectra were collected after cycles of 2 min of sputtering with Ar at $0.7 \mathrm{keV}$ until a constant composition was obtained. Spectral fitting and processing was performed using the CasaXPS Software Package. All high-resolution scans and survey scans were fitted to a Shirley background and all component peaks during the fitting process were generated by mixed Gaussian-Lorentz (GL) functions and fit according to the Scofield-based relative sensitivity factors (RSFs) built into CasaXPS.

Thermal Decomposition Solution Studies: Solutions of 1 and $\mathbf{2}$ were prepared by adding a known mass of compound to a known mass of $\mathrm{C}_{6} \mathrm{D}_{6}$. A drop of TMS was added as an internal standard. The concentrations for $\mathbf{1}$ and $\mathbf{2}$ were $7.8 \mathrm{mM}$ and $1.8 \mathrm{mM}$, respectively. The solutions were frozen in heavy walled NMR tubes in liquid $\mathrm{N}_{2}$ and flamed sealed under $100 \mathrm{mtorr}$ dynamic vacuum. The isothermal oven was illuminated with a $60 \mathrm{~W}$ incandescent light bulb and NMR tubes were wrapped with aluminum foil to exclude light. ${ }^{1} \mathrm{H}$ NMR spectra were measured on a $300 \mathrm{MHz}$ Avance 3 every $24 \mathrm{~h}$ after the tubes cooled to room temperature for 20 mins. The 2.5 ppm methylene peak in the ${ }^{1} \mathrm{H}$ NMR spectra was measured daily to track decomposition rates and products. 


\subsection{Supplemental Information}

Estimation of Vapour Pressure The procedure was followed as described in Ref. 34 with the modification that evaporation rates were measured from stepped isothermal experiments. The Langmuir equation was used to relate experimentally determined isothermal evaporation rates to vapour pressure:

$$
P=\frac{d m}{d t} \sqrt{\frac{T}{M}} \frac{\sqrt{2 \pi R}}{\alpha_{1}}
$$

where; $\mathrm{P}=$ vapour pressure

$\mathrm{T}=$ isothermal temperature

$\mathrm{dm} / \mathrm{dt}=$ evaporation rate determined from isothermal TGA

$\mathrm{M}=$ molecular mass

$\mathrm{R}=$ gas constant

$\alpha_{1}=$ vaporization coefficient

The vaporization coefficient, $\alpha_{1}$, accounts for the flowing gas environment of the

TG instrument. When considered along with $\sqrt{2 \pi R}$, the parameter $k=\frac{\sqrt{2 \pi R}}{\alpha_{1}}$ is defined as the material-independent part of Equation (1). The value for $k$ for the TGA instrument was determined from isothermal evaporation rates of benzoic acid and vapour pressure values determined from Antoine constants $(A=7.454, \mathrm{~B}=1820, \mathrm{C}=147.96)$. The value for $k$ was determined from the average of two stepped isothermal runs to be $65830 \mathrm{~kg}^{1 / 2}$ $\mathrm{m} \mathrm{s}^{-1} \mathrm{~K}^{-1 / 2}$. The stepped isothermal experiments were conducted between $60-120{ }^{\circ} \mathrm{C}$ with $10{ }^{\circ} \mathrm{C}$ increments and a ramp rate of $40{ }^{\circ} \mathrm{C} / \mathrm{min}$ was used between isothermal temperatures.

To validate this method, we compared our estimated vapor pressure data of $\mathrm{Cu}(\mathrm{tmhd})_{2}$ to known vapour pressure data (Colominas et al. J. Chem. Eng. Data 2001, 46, 446-450). Good agreement was obtained between these two sets of data. The temperature 
of 1 torr of vapour pressure was calculated to be $167^{\circ} \mathrm{C}$ and Colominas et al. measured 1 torr of vapor pressure at $152^{\circ} \mathrm{C}$.

The stepped isothermal experiments for $\mathbf{1}$ and $\mathbf{2}$ were conducted between 130-190 ${ }^{\circ} \mathrm{C}$ with $10{ }^{\circ} \mathrm{C}$ increments and a ramp rate of $40{ }^{\circ} \mathrm{C} / \mathrm{min}$ was used between isothermal temperatures. A $1 \mathrm{~cm} \mathrm{Pt} \mathrm{pan} \mathrm{with} \mathrm{a} \mathrm{surface} \mathrm{area} \mathrm{of} 0.785 \mathrm{~cm}^{2}$ was used for all experiments. The calibration constant, $k$, and Equation (1) were used to convert the isothermal evaporation rates to values for vapour pressure for $\mathbf{1}$ and $\mathbf{2}$ from which a 1 torr temperature could be obtained by extrapolation.

XPS and $\mathrm{Ar}^{+}$-sputtering: Chemical composition analysis of the $\mathrm{Au}$ and $\mathrm{Ag}$ films was carried out in a custom-built, ultra-high vacuum SPECS (GmbH) XPS system. Samples were mounted to an iron sample holder and held in place using molybdenum clips. For this study we used an Al Ka X-ray source operated at $\sim 14 \mathrm{keV}$ (26 mA) under a base pressure of $1 \times 10^{-10}$ Torr. Survey spectra $(0-1000 \mathrm{eV})$ and high-resolution scans of $\mathrm{Au}, \mathrm{Ag}, \mathrm{C}, \mathrm{N}$, and $\mathrm{O}$ regions were collected in SpecsLab2 (v. 2.7) software. Spectra were collected at a fixed sample holder angle of $0^{\circ}$ with the $\mathrm{x}$-ray probe-to-sample distance of $\sim 12 \mathrm{~mm}$. Optical parameters of the PHOIBOS hemispherical analyzer 3500 (HSA3500) and scanning parameters used in SpecsLab2 are found in the following table:

\begin{tabular}{lc|lcc}
\hline \multicolumn{2}{c}{ HSA3500 parameters } & \multicolumn{3}{c}{ scan mode (SpecsLab2) } \\
\hline entrance slit & $7 \mathrm{~mm} \times 20 \mathrm{~mm}$ & scan parameter & survey & component \\
\cline { 3 - 5 } aperture & medium & energy step (eV) & $0.2-0.4$ & $0.05-0.1$ \\
exit slit & open & dwell time (s) & 0.3 & $0.1-0.3$ \\
iris aperture & $32 \mathrm{~mm}$ & number of scans & 1 & $2-5$ \\
lens mode & medium & pass energy (eV) & 10 & 30 \\
analysis mode & $\begin{array}{c}\text { fixed analyzer } \\
\text { transmission }\end{array}$ & & & \\
\hline
\end{tabular}


The films were subjected to $\mathrm{Ar}^{+}$-sputtering cycles which last $2 \mathrm{~min} /$ cycle at a base pressure of $1 \times 10^{-5}$ Torr of Ar and $0.7 \mathrm{kV}$ filament potential. The sputtering depth of the $\mathrm{Ar}^{+}$is typically $10-20 \AA$ and was used to remove impurities such as $\mathrm{C}, \mathrm{O}$, and $\mathrm{N}$ that may have been initially present on the surface of the films. This would also yield the accurate bulk composition of the pristine $\mathrm{Au}$ and $\mathrm{Ag}$ films. Spectral fitting and processing was performed using the CasaXPS Software Package. All high-resolution scans and survey scans were fitted to a Shirley background and all component peaks during the fitting process were generated by mixed Gaussian-Lorentz (GL) functions and fit according to the Scofield-based relative sensitivity factors (RSFs) built into CasaXPS.

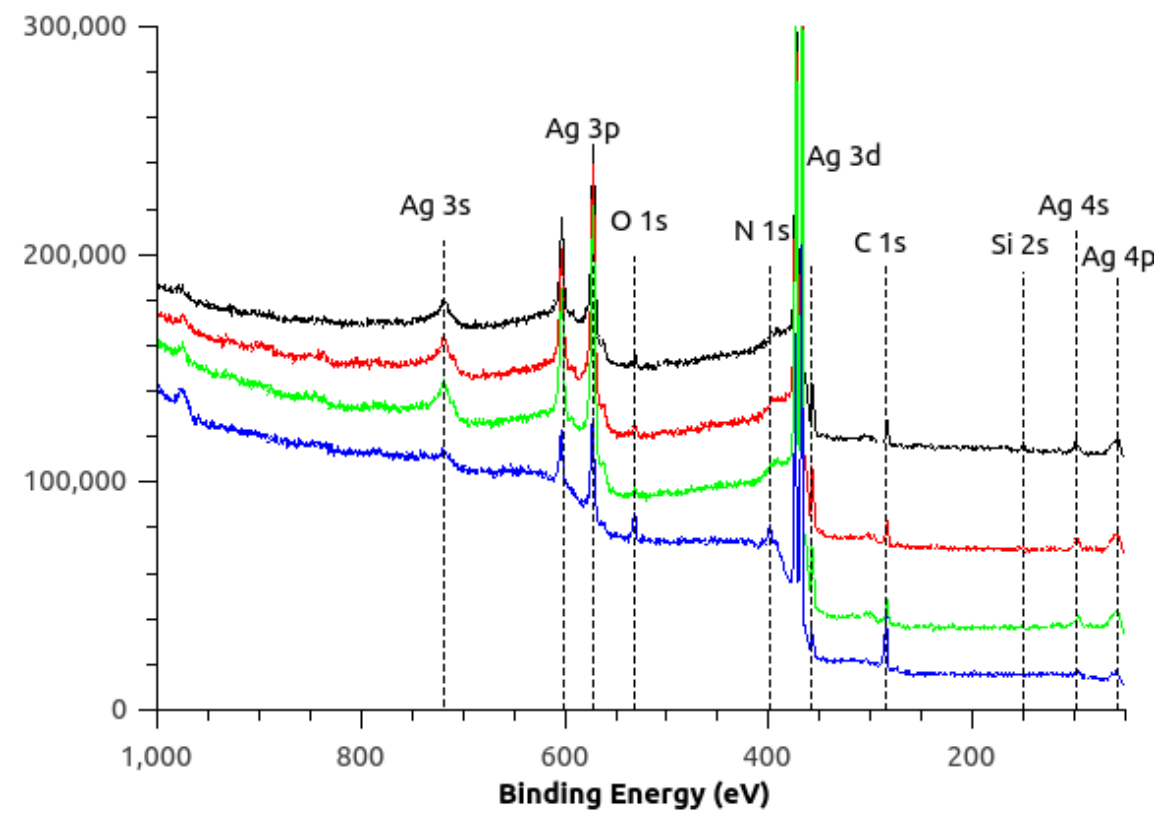

Figure 5.12 XPS survey spectrum of $\mathrm{Ag}$ on $\mathrm{Si}\left(\begin{array}{lll}1 & 0 & 0\end{array}\right)$ substrate as-deposited (blue), after 1 sputtering cycle (green), after 2 sputtering cycles (red), and after 3 sputtering cycles (black). $\mathrm{Ar}^{+}$sputtering cycles lasted $2 \mathrm{~min}$ and were done under base Ar pressures of $1 \mathrm{x}$ $10^{-5}$ Torr. 


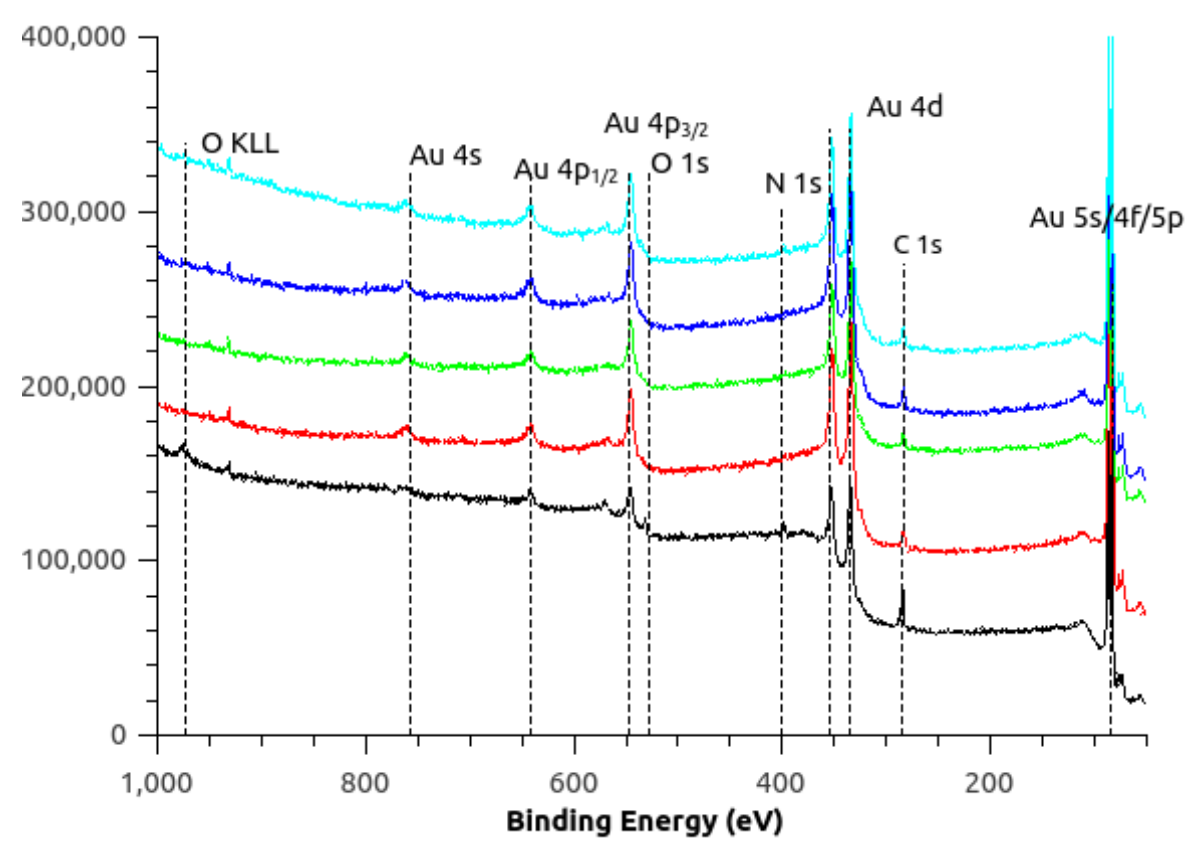

Figure 5.13 XPS survey spectrum of Au on Si (lll $\left.\begin{array}{ll}1 & 0\end{array}\right)$ substrate as deposited (black), after 1 sputtering cycle (red), after 2 sputtering cycles (green), after 3 sputtering cycles (blue), and after 4 sputtering cycles (cyan). $\mathrm{Ar}^{+}$sputtering cycles lasted $2 \mathrm{~min}$ and were done under Ar pressures of $1 \times 10^{-5}$ Torr.

\subsection{References}

1 Weber, W. H.; McCarthy, S. L. Phys. Rev. B. 1975, 12, 5643.

2 Ozbay, E. Science 2006, 311, 189.

3 Doppelt, P. Coord. Chem. Rev. 1998, 178-80, 1785.

4 George, S. M. Chem. Rev. 2010, 110, 111.

5 Miikkulainen, V.; Leskelä, M.; Ritala, M.; Puurunen, R. L. J. Appl. Phys. 2013, 113, 021301.

6 (a) Zaera, F. Coor. Chem. Rev. 2013 doi: 10.1016/j.ccr2013.04.006, (b) Lei, Y.; Lu, J.; Zhao, H.; Liu, B.; Low, K. -B.; Wu, T.; Libera, J. A.; Greely, J. P.; Chupas, P. J.; Miller, J. T.; Elam, J. W. J. Phys. Chem. C 2013, 117, 11141, (c) Geyer, S. M.; Methaapanon, R.; Shong, B.; Pianetta, P. A.; Bent, S. F. J. Phys. Chem. Lett. 2013, 4, 176. 
7 (a) Wenzel, T. J.; Williams, E. J.; Haltiwanger, R. C.; Sievers, R. E. Polyhedron 1985, 4, 369, (b) Martensson, P.; Carlsson, J.-O. J. Electrochem. Soc. 1998, 145 , 2926.

8 Park, K. H.; Marshall, W. J. J. Am. Chem. Soc. 2005, 127, 9330.

9 (a) Li, Z. W.; Rahtu, A.; Gordon, R. G. J. Electrochem. Soc. 2006, 153, C787,(b) Li, Z. W.; Barry, S. T.; Gordon, R. G. Inorg. Chem. 2005, 44, 1728, (c) Lim, B. S.; Rahtu, A.;Gordon, R. G. Nat. Mater. 2003, 2, 749,(d) Lim, B. S.; Rahtu, A.; Park, J. S.; Gordon, R. G. Inorg. Chem. 2003, 42, 7951.

10 Coyle, J. P.; Monillas, W. H.; Yap, G. P. A.; Barry, S. T. Inorg. Chem. 2008, 47, 683.

11 Park, J. W.; Jang, H. S.; Kim, M.; Sung, K.; Lee, S. S.; Chung, T.; Koo, S.; Kim, C. G.; Kim, Y. Inorg. Chem. Commun. 2004, 7, 463.

12 Grushin, V. V.; Marshall, W. J. Adv. Synth. Catal. 2004, 346, 1457.

13 (a) Yuan, Z.; Dryden, N. H.; Vittal, J. J.; Puddephatt, R. J. Chem. Mater. 1995, 7, 1696, (b) Chiong, H. A.; Daugulis, O. Organometallics 2006, 25, 4054.

14 International Technology Road for Semiconductors; 2011; http://www.itrs.net/.

15 Kariniemi, M.; Niinistö, J.; Hatanpää, T.; Kemell, M.; Sajavaara, T.; Ritala, M.; Leskelä, M. Chem. Mater. 2011, 23, 2901.

16 (a) Caldwell, J. D.; Glembocki, O. J.; Francisco, J. B.; Kariniemi, M. I.; Niinistö, J. T.; Hatanpaa, T. T.; Rendell, R. W.; Ukaegbu, M.; Ritala, M. K.; Prokes, A. M.; Hosten, C. M.; Leskelä, M. A.; Kasica, R. Opt. Express 2011, 19, 26056. (b) Prokes, S. M.; Glembocki, O. J.; Cleveland, E.; Caldwell, J. D.; Foos, E.; Niinistö, J.; Ritala, M. Appl. Phys. Lett. 2012, 100, 053106, (c) Cleveland, E. R.; Glembocki, O.; Prokes, S. M. Proceedings of the SPIE - Nanoepitaxy: Materials and Devices 2012, 8467, 84670H/1, (d) Prokes, S. M.; Glembocki, O. J.; Cleveland, E. Proceedings of the SPIE - Nanoepitaxy: Materials and Devices 2012, 8467, $84670 \mathrm{~F} / 1$

17 Whitehorne, T. J. J.; Coyle, J. P.; Mahmood, A.; Monillas, W. H.; Yap, G. P. A.; Barry, S. T. Eur. J. Inorg. Chem. 2011, 3240

18 (a) Shao, L. -Y.; Coyle, J. P.; Barry, S. T.; Albert, J. Opt. Mater. Express 2011, 1, 128, (b) Zhou, W.; Mandia, D. J.; Griffiths, M. B. E.; Bialiayeu, A.; Zhang, Y.; Gordon, P. G.; Barry, S. T.; Albert, J. Opt. Express 2013, 21, 245 
19 (a) James, A. M.; Laxman, R. K.; Fronczek, F. R.; Maverick, A. W. Inorg. Chem. 1998, 37, 3785, b)Hitchcock, P. B.; Lappert, M. F.; Pierssens, L. J. -M. Chem.

Commun. 1996, 1189, (c) Bunge, S. D.; Just, O.; Rees, W. S. Jr. Angew. Chem. Int. Ed. 2000, 39, 3082.

20 Grodzicki, A.; Łakomska, I.; Piszczek, P.; Szymańska, I.; Szłyk, E. Coord. Chem. Rev. 2005, 249, 2232.

21 Bollmann, D.; Merkel, R.; Klumpp, A. Microelectron. Eng. 1997, 37-8, 105

22 Norman, J. A.T.; Perez, M.; Schulz, S. E.; Waechtler, T. Microelectron. Eng. 2008, 85,2159

23 Gao, L.; Härter, P.; Linsmeier, Ch.; Wiltner, A.; Emling, R.; Schmitt-Landsiedel, D. Microelectron. Eng. 2005, 82, 296

24 Chi, K.-M.; Lu, Y.-H. Chem. Vap. Dep. 2001, 7, 117.

25 Yuan, Z.; Dryden, N. H.; Li, X.; Jagadese, J. V.; Puddephatt, R. J. J. Mater. Chem. 1995, 5, 303.

26 Szlyk, E.; Piszczek, P.; Lakomska, I.; Grodzicki, A.; Szatkowski, J.; Blaszczyk, T. Chem. Vap. Dep. 2000, 6, 105.

27 (a) Banaszak Holl, M. M.; Seidler, P. F.; Kowalczyk, S. P.; McFeely, F. R. Inorg. Chem. 1994, 33, 510, (b) Jansen, F.; Kruck, T. Adv. Mater. 1995, 7, 298, (c) Au, R. H. W.; Puddephatt, R. J. Chem. Vap. Dep. 2007, 13, 20.

28 (a) Larson, C. E.; Baum, T. H.; Jackson, R. L. J. Electrochem. Soc. 1987, 134, 266, (b) Bessonov, A. A.; Morozova, N. B.; Gelfond, N. V.; Semyannikov, P. P.; Trubin, S. V.; Shevtsov, Yu. V.; Shubin, Yu. V.; Igumenov, I. K. Surf. Coat. Technol. 2007, 210, 9099, (c) Bessonov, A. A.; Morozova, N. B.; Gelfond, N. V.; Semyannikov, P. P.; Baidina, I. A.; Trubin, S. V.; Shevtsov, Yu. V.; Igumenov, I. K. J. Organomet. Chem. 2008, 693, 2572, (d) Parkhomenko, R. G.; Morozova, N. B.; Zharkova, G. I.; Shubin, Y. V.; Trubin, S. V.; Kriventsov, V. V.; Kuchumov, B. M.; Koretskaya, T. P.; Igumenov, I. K. Chem. Vap. Dep. 2012, 18, 336, (e) Parkhomenko, R. G.; Turgambaeva, A. E.; Morozova, N. B.; Trubin, S. V.; Krisyuk, V. V.; Igumenov, I. K. Chem. Vap. Dep. 2013, 19, 38.

29 Coyle, J. P.; Johnson, P.; DiLabio, G.; Müller, J.; Barry, S. T. Proceedings of the AVS Atomic Layer Deposition Conference 2009. 
30 Coyle, J. P.; Johnson, P.A.; DiLabio, G. A.; Barry, S. T.; Müller, J. Inorg. Chem. 2010, 49, 2844.

31 Coyle, J. P.; Kurek, A.; Pallister, P. J.; Sirianni, E. R.; Yap, G. P. A.; Barry, S. T. Chem. Commun. 2012, 48, 10440.

32 Ziffle, L. C.; Kenney, A. P.; Barry, S. T.; Müller, J. Polyhedron 2008, 27, 1832.

33 JCPDS Card File No. 00-004-0784 (Au) and 01-089-3722 (Ag).

34 Coyle, J. P.; Pallister, P. J.; Kurek, A.; Sirianni, E. R.; Yap, G. P. A.; Barry, S. T. Inorg. Chem. 2013, 52, 910.

35 Kunte, G. V.; Shivashankar, S. A.; Umarji, A. M. Meas. Sci. Technol. 2008, 19, 025704 .

36 (a) Ma, Q.; Guo, H.; Gordon, R. G.; Zaera, F. Chem. Maters. 2011, 23, 3325, (b) Kim, T.; Yao, Y.; Coyle, J. P.; Barry, S. T.; Zaera, F. Chem. Maters. 2013, 25, 3630 .

37 Gordon, P. G.; Ward, M. J.; Heikkila, M. J.; Monillas, W. H.; Yap, G. P. A.; Ritala, M.; Leskelä, M.; Barry, S. T. Dalton Trans. 2011, 40, 9425.

38 Usón, R.; Laguna, A.; Laguna, A. M.; Briggs, D. A.; Murray, H. H.; Fackler, J. P. Inorg. Synth. 1989, 26, 85.

39 Sheldrick, G.M. Acta Cryst. 2008, A64, 112-122.

40 Horcas, I.; Fernandez, R.; Gomez-Rodriguez, J. M.; Colchero, J.; Gomez-Herrero, J.; Baro, A. M. Rev. Sci. Instrum. 2007, 78, 013705. 


\section{Chapter: Deposition of Copper by Plasma-Enhanced Atomic Layer Deposition Using a Novel N-Heterocyclic Carbene Precursor}

Modified from original manuscript published as:

Jason P. Coyle ${ }^{1}$, Gangotri Dey ${ }^{2}$, Eric R. Sirianni ${ }^{3}$, Marianna L. Kemell ${ }^{4}$, Glenn P. A. Yap ${ }^{3}$, Mikko Ritala $^{4}$, Markku Leskelä ${ }^{4}$, Simon D. Elliott ${ }^{2}$, Sean T. Barry "Deposition of Copper by PlasmaEnhanced Atomic Layer Deposition Using a Novel N-Heterocyclic Carbene Precursor" Chem. Mater. 2013 25, 1132-1138

${ }^{1}$ Department of Chemistry, Carleton University, 1125 Colonel By Drive, Ottawa, K1S 5B6, Canada.

${ }^{2}$ Tyndall National Institute, University College Cork, Dyke Parade, Cork, Ireland

${ }^{3}$ Department of Chemistry \& Biochemistry, University of Delaware, Newark, DE 19716, United States

${ }^{4}$ Laboratory of Inorganic Chemistry, Department of Chemistry, University of Helsinki P.O. Box 55, FI-00014 Helsinki, Finland

\subsection{Abstract}

Two novel N-heterocyclic carbene (NHC)-containing copper(I) amides are reported as atomic layer deposition (ALD) precursors. 1,3-diisopropyl-imidazolin-2ylidene copper hexamethyldisilazide (1) and 4,5-dimethyl-1,3-diisopropyl-imidazol-2ylidene copper hexamethyldisilazide (2) were synthesized and structurally characterized. The thermal behaviour of both compounds was studied by thermogravimetric analysis (TGA), and they were both found to be reasonably volatile compounds. Compound $\mathbf{1}$ had no residual mass in the TGA and showed long-term stability at temperatures as high as $130{ }^{\circ} \mathrm{C}$, while 2 had a residual mass of $7.4 \%$. Copper metal with good resistivity was deposited using 1 by plasma-enhanced atomic layer deposition. The precursor demonstrated self-limiting behaviour indicative of ALD, and gave a growth rate of 0.2 $\AA$ Acycle. Compound $\mathbf{2}$ was unsuccessful as an ALD precursor under similar conditions. Density functional theory calculations showed that both compounds adsorb dissociatively 
onto a growing copper film as long as there is some atomic roughness, via cleavage of the $\mathrm{Cu}$-carbene bond.

\subsection{Introduction}

Copper metal remains an interesting topic for chemical vapour deposition (CVD) and atomic layer deposition (ALD) due to its use in microelectronics, primarily as an interconnect. ${ }^{1}$ Several potential copper(I) and copper(II) precursors have been reported for chemical vapor deposition (CVD) and atomic layer deposition (ALD), including $\beta$ diketonates, ${ }^{2} \beta$-diketiminates, ${ }^{3}$ amidinates, ${ }^{4}$ guanidinates, ${ }^{5}$ aminoalkoxides, ${ }^{6}$ and pyrrolylaldiminates. ${ }^{7}$ Of these precursors, the exclusively N-bonded ligands are interesting due to their lack of $\mathrm{Cu}-\mathrm{O}$ bonds, making them less susceptible to oxygen inclusion in the deposited copper film as well as in the barrier and adhesion layers.

ALD has been proposed as an alternative method to CVD for depositing conformal, ultra-thin films at comparatively lower temperatures. ALD is similar to CVD except that the substrate is sequentially exposed to one reactant at a time, or one dose of a reactant at a time. Conceptually, it is a simple process: a first reactant is introduced to a heated substrate whereby it forms a monolayer on the surface of the substrate. Excess reactant is pumped out (e.g., evacuated). Next a second reactant is introduced and reacts with the existing monolayer to form a sub-monolayer of a desired reaction product through a self-limiting surface reaction. The process is self-limiting since the deposition reaction halts once the initially adsorbed (physisorbed or chemisorbed) monolayer of the first reactant has fully reacted with the second reactant. Finally, the excess second reactant is evacuated. This sequence constitutes one deposition cycle. The desired film thickness is obtained by repeating deposition cycles as necessary. As is apparent, the 
sequential layer-by-layer nature of ALD has the disadvantage of being slower than some other deposition techniques. However it is this cycle of building up highly uniform layers one at a time that allows ALD to produce films of a surface uniformity, smoothness and thinness that is impossible to achieve with other techniques. This makes ALD uniquely valuable in demanding coating applications. In the specific application of ALD used for this study, the "second reactant" in this case is a hydrogen plasma, generated remotely from the growing surface from dihydrogen carried by argon. This plasma is screened by a grounded grid, filtering out ions from the plasma, and permitting only the radicals (and light) produced in the plasma to impinge on the surface. The role of the the hydrogen plasma is to scour off the ligand system, as well as reducing the copper surface species to metal. Given the complex nature of the plasma, the mechanistic reaction at the surface is likely complex. An excellent review of plasma ALD processes can be found here. ${ }^{8}$

It is interesting that copper amides have not been prevalent precursors for ALD. It is known that copper(I) amides typically produce a tetrameric structure, ${ }^{9}$ and copper(I) amides are more thermally stabile than alkyl or alkoxo species. ${ }^{10}$ Thus, it has been difficult to isolate a thermally stable copper amide precursor that exhibits sufficient volatility to be considered for CVD or ALD. However, monomeric copper(I) species are well-studied in catalysis. Specifically, copper(I) NHCs are well-known to exhibit good thermal stability during $\mathrm{C}-\mathrm{H}$ bond activation ${ }^{11}$ and can drastically increase the thermal stability in sensitive $\mathrm{Cu}(\mathrm{I})$ species. ${ }^{12,13}$ Thus, the synthesis and thermal characterization of copper(I) amides stabilized by NHCs was undertaken to examine their suitability as 
ALD precursors. This design strategy is common for group 11 precursors, and recent success has been shown for the plasma enhanced ALD of Ag films. ${ }^{14}$
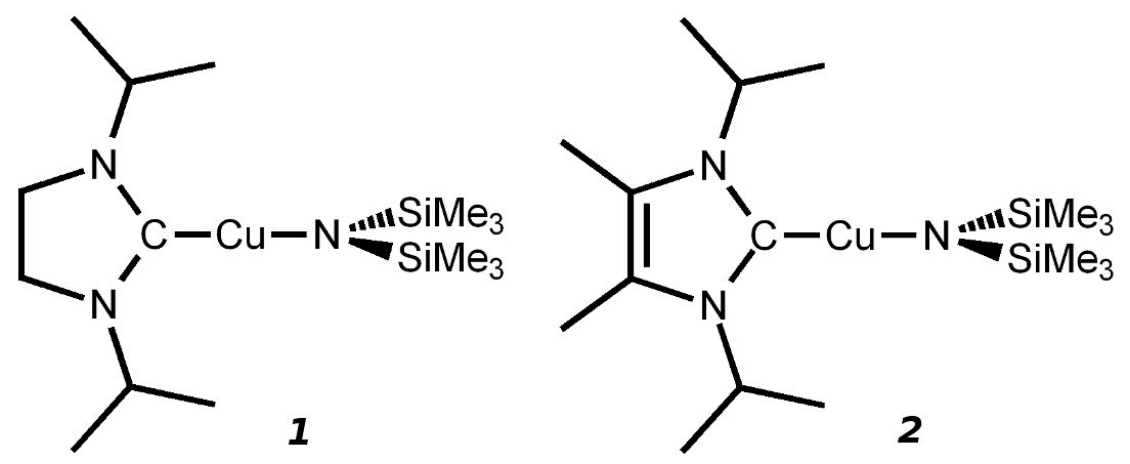

Figure 6.1 The structures of 1,3-diisopropyl-imidazolin-2-ylidene copper hexamethyldisilazide (1) and 4,5-dimethyl-1,3-diisopropyl-imidazol-2-ylidene copper hexamethyldisilazide (2).

Herein is reported novel copper(I) NHCs: 1,3-diisopropyl-imidazolin-2-ylidene copper hexamethyldisilazide (1) and 4,5-dimethyl-1,3-diisopropyl-imidazol-2-ylidene copper hexamethyldisilazide (2) (Figure 6.1). The choice of ligands was paramount for the design of this new copper precursor. Preliminary screening showed that hexamethyldisilazide $\left(\mathrm{N}\left(\mathrm{SiMe}_{3}\right)_{2}\right)$ provided excellent thermal stability to $\mathrm{Cu}(\mathrm{I})$, where alkyl amides are known to allow plating of copper metal. ${ }^{10}$ It was less clear from the outset whether an unsaturated NHC (imidazol-2-ylidene) or saturated NHC (imidazolin2-ylidene) would allow for better precursor properties and for better metal deposition. Preliminary screening of unsaturated NHCs showed that an imidazol-2-ylidene with a methyl-substituted backbone provided a more thermally stable copper compound than an unsubstituted imidazol-2-ylidene. Preliminary screening showed backbone substitution of imidazolin-2-ylidenes proved to be unnecessary as such NHCs afforded copper compounds with sufficient thermal stability. Interestingly, our preliminary screening led 
us to choose NHCs with different stabilities towards dimerization; the dimer from the carbene in $\mathbf{2}$ is unknown, whereas the dimer from the carbene in $\mathbf{1}$ is a volatile olefin. ${ }^{15}$

Some $\mathrm{Cu}(\mathrm{I})$ precursors are well known to undergo disproportionation reactions to $\mathrm{Cu}(0)$ and $\mathrm{Cu}(\mathrm{II})$, which has been exploited in the CVD of copper metal films. ${ }^{16}$ This process relies on the clean purging from the deposition zone of the $\mathrm{Cu}(\mathrm{II})$ product along with any stabilizing Lewis base employed in the $\mathrm{Cu}(\mathrm{I})$ precursor. $\mathrm{Cu}(\mathrm{II})$ hexamethyldisilazide compounds are unknown and $\mathrm{Cu}(\mathrm{II})$ amide compounds are exceptionally rare. ${ }^{17}$ The attempted synthesis of $\mathrm{Cu}\left[\mathrm{N}\left(\mathrm{SiMe}_{3}\right)_{2}\right]_{2}$ results in the isolation of $\left[\mathrm{CuN}\left(\mathrm{SiMe}_{3}\right)_{2}\right] 4 \cdot{ }^{18} \mathrm{CVD}$ of copper metal films from $\left[\mathrm{CuN}\left(\mathrm{SiMe}_{3}\right)_{2}\right]_{4}$ have been reported at $200^{\circ} \mathrm{C}$ under a flow of $\mathrm{H}_{2}$ without any indication of a disproportionation component to film growth. ${ }^{18}$ Both of the NHCs used in $\mathbf{1}$ and $\mathbf{2}$ are volatile at room temperature when not coordinated; however their affinities for a copper surface are investigated herein.

Additionally, explicit atomic-scale insight into ALD chemistry has been obtained through simulations using Density Functional Theory (DFT). ${ }^{19}$ Most DFT studies have been related to the ALD of binary compounds such as $\mathrm{HfO}_{2}{ }^{20}$ and $\mathrm{Al}_{2} \mathrm{O}_{3}$, ${ }^{21}$ but there are relatively few simulations on the ALD of pure metals (e.g. $\mathrm{Ni}^{22}$ and $\mathrm{Co}^{23}$ ). Possible $\mathrm{Cu}$ ALD reactions have been computed by Mårtensson et al. for $\mathrm{CuCl}$ as the precursor and $\mathrm{H}_{2}{ }^{24}$ as the reducing agent. The formation of $\mathrm{Cu}$ from amidinate ${ }^{25}$ and $\beta$-diketonate ${ }^{26,27}$ based precursors has also been simulated. Recently, Dey et al. have computed a detailed mechanism for $\mathrm{Cu} \mathrm{ALD}$ using $\mathrm{ZnEt}_{2}$ as the reducing agent, ${ }^{28}$ following experiments by Lee et al. ${ }^{29}$ Here we use DFT to compare the probable adsorption mechanism of the carbene-based $\mathrm{Cu}$ precursors $\mathbf{1}$ and $\mathbf{2}$. 


\subsection{Results and Discussion}

Both compounds can be made by simple salt metathesis from the NHC copper chloride and $\mathrm{Et}_{2} \mathrm{O} \cdot \mathrm{LiN}\left(\mathrm{SiMe}_{3}\right)_{2}$. The resulting ${ }^{1} \mathrm{H}$ spectra were simple and reflected the expected formulations in both cases. Compound $\mathbf{1}$ easily underwent sublimation under reduced atmosphere at $90^{\circ} \mathrm{C}$, and was sublimed quantitatively. It melted at $51^{\circ} \mathrm{C}$, which is beneficial for a vapour phase precursor: having the precursor as a liquid during the deposition process ensures a uniform production of vapour pressure. Thermogravimetric analysis (TGA) showed the onset of volatility at $91{ }^{\circ} \mathrm{C}$ and a residual mass of $0 \%$ (Figure 6.2, black trace). Compound 2 could also be quantitatively sublimed at $95^{\circ} \mathrm{C}$ and had a melting point of $119^{\circ} \mathrm{C}$. TGA showed an onset of volatility at $87^{\circ} \mathrm{C}$, but with a residual mass of $7.4 \%$. Since the mass of copper in 2 comprises $15.72 \%$ of the total molecular mass, this compound was possibly undergoing volatilization and decomposition over the thermal range of the TGA.
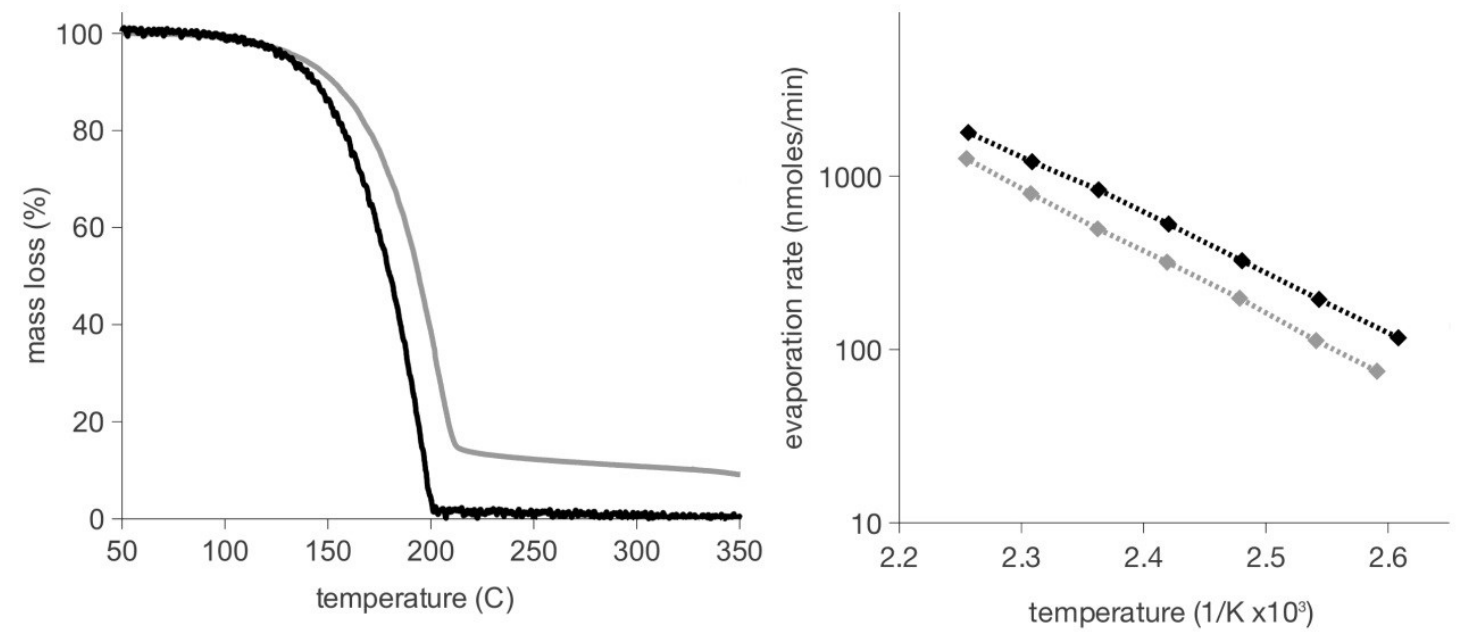

Figure 6.2 Thermogravimetric analyses and evaporation rates of $\mathbf{1}$ (black) and $\mathbf{2}$ (grey) run at $10{ }^{\circ} \mathrm{C} / \mathrm{min}$ ramp rate, with $10{ }^{\circ} \mathrm{C}$ increments for the stepped isotherm used to determine evaporation rate. 
It was possible to isolate crystals of both $\mathbf{1}$ and $\mathbf{2}$ that were sufficient for structural analysis (Figure 6.3, Table 6.1). In both cases, the copper is in a linear geometry with the ligands staggered and roughly orthogonal. The $\mathrm{Cu}-\mathrm{N}$ bonds $(1.84 \AA$ for $\mathbf{1}$ and $1.87 \AA$ for 2) are shorter than reported for the copper(I) hexamethyldisilazide tetramer (1.92 - 1.93 $\AA),{ }^{18}$ but similar to copper alkyl amide compounds. ${ }^{9}$ The longer amide contact in 2 might be due to the steric bulk of the backbone methyl groups forcing the carbene's isopropyl groups to orient toward the amide moiety, in turn forcing back the amide group. The copper-carbon bonds fall in the range of other monomeric, linear N-bonded $\mathrm{Cu}-\mathrm{NHC}$ complexes, ${ }^{30,12}$ suggesting that the shortened $\mathrm{Cu}-\mathrm{N}$ bond might be due to enhanced $\pi$ donation to the copper. This also suggests that the copper amide bond might be stabilized to cleavage upon adsorption of the compound at a growing copper surface, suggesting that the carbene will be lost upon chemisorption.
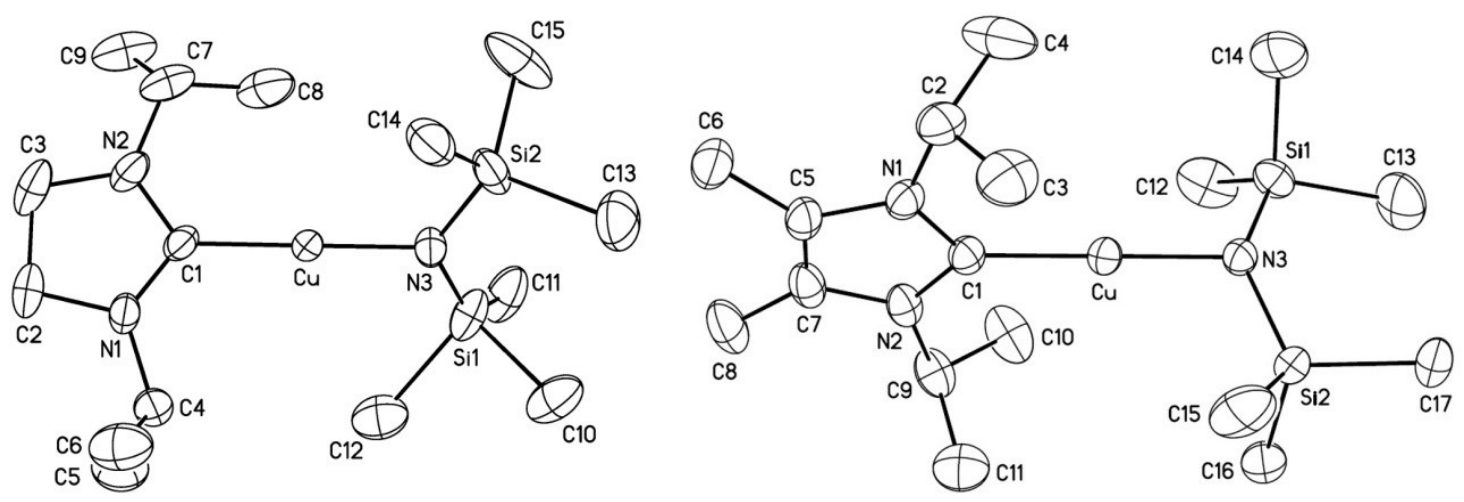

Figure 6.3 The X-ray structure of $\mathbf{1}$ (left) and 2, (right), with H-atoms and minor disordered contributions omitted for clarity and at $30 \%$ probability ellipsoids. 
Table 6.1 Selected bond lengths and bond angles for the structures of $\mathbf{1}$ and $\mathbf{2}$.

\begin{tabular}{|c|c|c|c|c|c|}
\hline \multicolumn{3}{|c|}{ Compound 1} & \multicolumn{3}{|c|}{ Compound 2} \\
\hline & experimental & computational & & experimental & computational \\
\hline \multicolumn{6}{|c|}{ selected bond lengths $(\AA)$} \\
\hline $\mathrm{Cu}-\mathrm{N} 3$ & $1.836(4)$ & 1.869 & $\mathrm{Cu}-\mathrm{N} 3$ & $1.870(2)$ & 1.869 \\
\hline $\mathrm{Cu}-\mathrm{C} 1$ & $1.870(5)$ & 1.897 & $\mathrm{Cu}-\mathrm{C} 1$ & $1.881(2)$ & 1.897 \\
\hline $\mathrm{N} 1-\mathrm{C} 1$ & $1.330(7)$ & 1.356 & $\mathrm{~N} 1-\mathrm{C} 1$ & $1.360(3)$ & 1.367 \\
\hline $\mathrm{N} 1-\mathrm{C} 2$ & $1.472(7)$ & 1.470 & $\mathrm{~N} 1-\mathrm{C} 5$ & $1.404(3)$ & 1.397 \\
\hline $\mathrm{N} 2-\mathrm{C} 1$ & $1.336(6)$ & 1.356 & $\mathrm{~N} 2-\mathrm{C} 1$ & $1.365(3)$ & 1.372 \\
\hline $\mathrm{N} 2-\mathrm{C} 3$ & $1.444(9)$ & 1.471 & $\mathrm{~N} 2-\mathrm{C} 7$ & $1.394(3)$ & 1.399 \\
\hline Si1-N3 & $1.680(6)$ & 1.742 & $\mathrm{~N} 3-\mathrm{Si} 2$ & $1.692(2)$ & 1.742 \\
\hline Si2-N3 & $1.701(5)$ & 1.742 & N3-Si1 & $1.697(2)$ & 1.741 \\
\hline $\mathrm{C} 2-\mathrm{C} 3$ & $1.531(12)$ & 1.538 & $\mathrm{C} 5-\mathrm{C} 7$ & $1.338(4)$ & 1.384 \\
\hline \multicolumn{6}{|c|}{ selected bond angles (deg) } \\
\hline $\mathrm{N} 3-\mathrm{Cu}-\mathrm{C} 1$ & $179.2(2)$ & 178.3 & $\mathrm{~N} 3-\mathrm{Cu}-\mathrm{C} 1$ & $178.60(9)$ & 175.5 \\
\hline $\mathrm{C} 1-\mathrm{N} 1-\mathrm{C} 2$ & $114.2(5)$ & 112.1 & $\mathrm{C} 1-\mathrm{N} 1-\mathrm{C} 5$ & $111.0(2)$ & 111.4 \\
\hline $\mathrm{C} 1-\mathrm{N} 2-\mathrm{C} 3$ & $114.7(6)$ & 112.5 & $\mathrm{C} 1-\mathrm{N} 2-\mathrm{C} 7$ & $111.6(2)$ & 111.4 \\
\hline Si1-N3-Si2 & $128.2(3)$ & 128.2 & Si2-N3-Si1 & $131.26(13)$ & 128.1 \\
\hline $\mathrm{Si} 1-\mathrm{N} 3-\mathrm{Cu}$ & $116.5(3)$ & 116.0 & $\mathrm{Si} 1-\mathrm{N} 3-\mathrm{Cu}$ & $113.19(11)$ & 113.0 \\
\hline $\mathrm{Si} 2-\mathrm{N} 3-\mathrm{Cu}$ & 115.3(3) & 115.1 & $\mathrm{Si} 2-\mathrm{N} 3-\mathrm{Cu}$ & 111.21(11) & 113.0 \\
\hline $\mathrm{N} 1-\mathrm{C} 1-\mathrm{N} 2$ & $106.6(5)$ & 107.9 & $\mathrm{~N} 1-\mathrm{C} 1-\mathrm{N} 2$ & $103.9(2)$ & 104.5 \\
\hline $\mathrm{N} 1-\mathrm{C} 1-\mathrm{Cu}$ & $127.0(4)$ & 128.0 & $\mathrm{~N} 1-\mathrm{C} 1-\mathrm{Cu}$ & $127.26(19)$ & 130.6 \\
\hline $\mathrm{N} 2-\mathrm{C} 1-\mathrm{Cu}$ & $126.3(5)$ & 128.0 & $\mathrm{~N} 2-\mathrm{C} 1-\mathrm{Cu}$ & 128.88(19) & 130.6 \\
\hline
\end{tabular}

A "stepped isotherm" TGA was employed to establish the evaporation rate and vapour pressures of these compounds. A stepped isotherm is a thermogravimetric experiment where the temperature is ramped by $10{ }^{\circ} \mathrm{C}$ and held for 10 minutes while the compound volatilizes; the number of steps gives the number of data points from which a Clausius-Clapeyron curve can be constructed. This experiment can be used to relate the mass lost as vapour to the Langmuir equation, to establish vapour pressure as a function 
of temperature. ${ }^{31}$ Compound $\mathbf{1}$ was shown to be more volatile at lower temperatures, and to have 1 Torr of vapour pressure at $149^{\circ} \mathrm{C}$ (using benzoic acid as a standard). ${ }^{32}$ Compound $\mathbf{2}$ had a slightly lower volatility, and gave 1 Torr of vapour pressure at 158 ${ }^{\circ} \mathrm{C}$. However, both of these compounds can be expected to give reasonably high vapour pressures at normal bubbler temperatures, and the similarity of the slopes of their evaporation curves suggests that they experience similar intermolecular attraction during evaporation.

Since the TGA of 1 showed no thermal decomposition, a thermal "delivery stability" test was performed to determine the long-term stability of this compound at reasonable bubbler temperatures. It is desirable for ALD precursors to demonstrate stability under bubbler conditions to ensure predictable and consistent vapour pressures delivered with each pulse. The thermal stress test used a stainless steel vessel which was heated in an isothermal oven. Samples were taken using a glovebox every $48 \mathrm{~h}$ for 2 weeks, and the samples were analysed by TGA and ${ }^{1} \mathrm{H}$ NMR. A $90{ }^{\circ} \mathrm{C}$ oven temperature was chosen as this was the delivery temperature in plasma-enhanced (PE) ALD experiments described below. The TGA showed an increasing trend in residual mass from $2 \%$ to $3 \%$ over two weeks, which is an excellent thermal stability at delivery temperatures. The ${ }^{1} \mathrm{H}$ NMR spectra measured on day 1 and day 14 both showed only peaks for $\mathbf{1}$. This delivery stability test was repeated using an oven temperature of 130 ${ }^{\circ} \mathrm{C}$. Sampling over two weeks showed the residual mass increasing from $1 \%$ to $3 \%$. Again, ${ }^{1} \mathrm{H}$ NMR spectra corroborated the excellent thermal stability displayed at $130{ }^{\circ} \mathrm{C}$. A delivery stability test was not performed for $\mathbf{2}$, as decomposition was evident from preliminary TGA. 
Compound 1 was successful at depositing copper metal using PE-ALD. A typical experiment saw $0.3-0.5 \mathrm{~g}$ of 1 loaded into a Beneq TFS 200 reactor equipped with a capacitively coupled plasma source. The substrate was heated to $225{ }^{\circ} \mathrm{C}$ and the precursor was heated to $90{ }^{\circ} \mathrm{C}$ to ensure sufficient vapour of 1 reaching the deposition chamber. A pulse of 1 was followed by a 3 s purge with nitrogen gas, alternating with a $6 \mathrm{~s}$ pulse of hydrogen plasma and a second purge. Varying the pulse length of $\mathbf{1}$ allowed a saturation curve to be collected (Figure 6.4). The growth rate plateaued at $0.2 \AA$ /cycle after

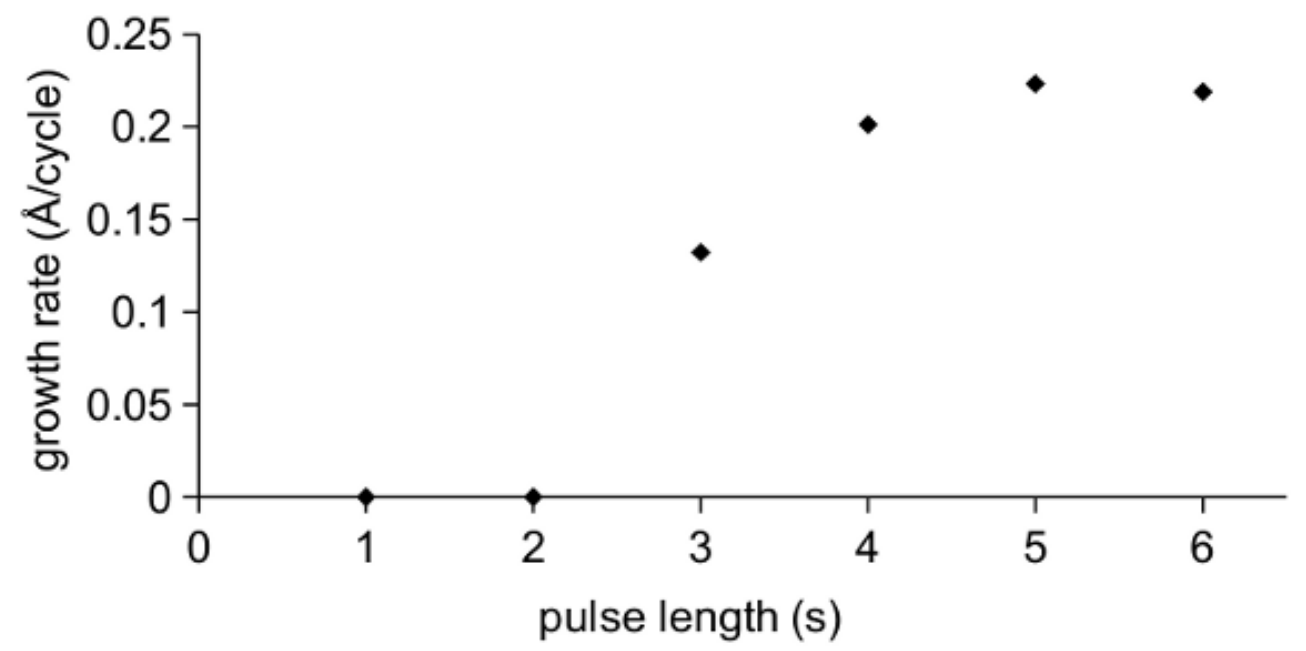

Figure 6.4 ALD saturation curve for 1 as the pulse length of 1 was varied.

4 seconds, corresponding to about $10 \%$ of a monolayer of $\mathrm{Cu}$. ALD-grown copper has been reported with growth rates of $0.12 \AA$ /cycle (on Ru), ${ }^{33} 0.18 \AA$ /cycle,${ }^{34}$ and 0.90 $\AA /$ cycle $^{35}$, showing this growth rate to be within this range. Energy dispersive X-ray spectroscopy (EDS) showed the resulting material to be copper metal, with some minor carbon impurities $(<5 \%)$. Since the thicknesses were measured using the k-ratios from EDS, the growth from $1 \mathrm{~s}$ and $2 \mathrm{~s}$ pulse lengths gave no thickness. However, scanning electron microscopy showed nucleation of copper nanoparticles (Figure 6.5). It was 
obvious that the deposited copper was crystalline over all deposition ranges, and X-ray diffraction showed typical copper signals, and demonstrated the absence of copper oxide crystalline phases. Many of the deposited films were too thin and non-continuous for resistivity measurements, but the film deposited with a $5 \mathrm{~s}$ pulse had a thickness of $35 \mathrm{~nm}$ and gave a resistivity of $11.23 \mu \Omega \cdot \mathrm{cm}$. This is not unusual for the deposition of copper metal on silicon: the high mobility of copper atoms on the growing surface result in an island growth mechanism that causes non-uniformity.
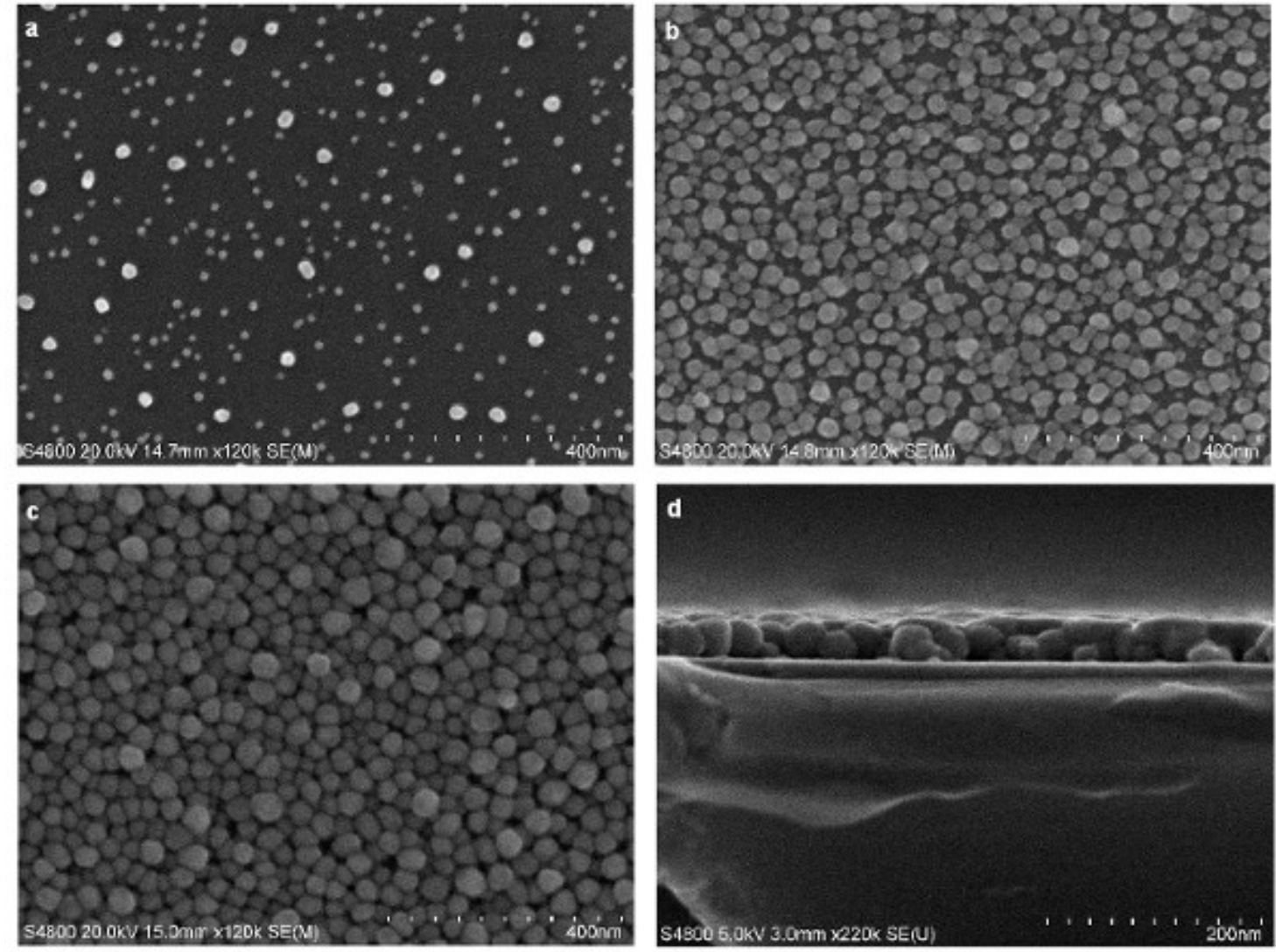

Figure 6.5 Scanning electron micrographs of deposited copper films. (a) Plan view of copper nanoparticles deposited using a $1 \mathrm{~s}$ pulse length. (b) Plan view of crystalline copper deposited using a $4 \mathrm{~s}$ pulse length. (c) Plan view of crystalline copper deposited using a 6 s pulse length. (d) A profile of crystalline copper deposited using a $6 \mathrm{~s}$ pulse length. 
Compound $\mathbf{2}$ could not be used under any tested conditions as a copper metal precursor. The resulting depositions were not metallic, but showed an interference colour pattern similar to non-uniform deposition of a transparent material. A typical "blank" experiment was performed where by the hydrogen pulse was not activated to plasma. No film growth was observed during this experiment for either $\mathbf{1}$ or $\mathbf{2}$. The difference in the deposition results between $\mathbf{1}$ and $\mathbf{2}$ was attributed to the NHC, since the rest of the molecule is unchanged.

DFT calculations were carried out on these precursors in the gas phase and adsorbed to model copper surfaces. The computed structures were in good agreement with the X-ray structural analysis, with all bond lengths agreeing within $5 \mathrm{pm}$ (Table 6.1). The dissociation energy of each ligand from the copper centre was calculated to determine which bond was likely to break upon chemisorption at a copper surface. The chemical equations considered were heterolysis of the amide bond (1), homolysis of the amide bond (2), and loss of the NHC (3):

$$
\begin{aligned}
& \mathrm{NHC}-\mathrm{Cu}(\mathrm{I})-\mathrm{N}\left(\mathrm{SiMe}_{3}\right)_{2} \rightarrow[\mathrm{NHC}-\mathrm{Cu}(\mathrm{I})]^{+}+\left[\mathrm{N}\left(\mathrm{SiMe}_{3}\right)_{2}\right]^{-} \\
& \mathrm{NHC}-\mathrm{Cu}(\mathrm{I})-\mathrm{N}\left(\mathrm{SiMe}_{3}\right)_{2} \rightarrow[\mathrm{NHC}-\mathrm{Cu}(0)]+\left[\mathrm{N}\left(\mathrm{SiMe}_{3}\right)_{2}\right]^{\cdot} \\
& \mathrm{NHC}-\mathrm{Cu}(\mathrm{I})-\mathrm{N}\left(\mathrm{SiMe}_{3}\right)_{2} \rightarrow \mathrm{NHC}+\mathrm{Cu}(\mathrm{I})-\mathrm{N}\left(\mathrm{SiMe}_{3}\right)_{2}
\end{aligned}
$$

In compounds $\mathbf{1}$ and $\mathbf{2}$, it was found that the copper-nitrogen bond (Eqs. 1 and 2) was much stronger than the copper-C bond to NHC (Eq. 3), (646 and $428 \mathrm{~kJ} / \mathrm{mol}$ vs. 293 $\mathrm{kJ} / \mathrm{mol}$ for 1; 636 and $427 \mathrm{~kJ} / \mathrm{mol}$ vs. $298 \mathrm{~kJ} / \mathrm{mol}$ for 2). Thus, the amide will remain bound to the $\mathrm{Cu}$ atom during adsorption, which likely forms new $\mathrm{Cu}-\mathrm{Cu}$ bonds with the 
surface, while the carbene may dissociate from the adsorbing molecule and bond separately to the surface or evaporate.
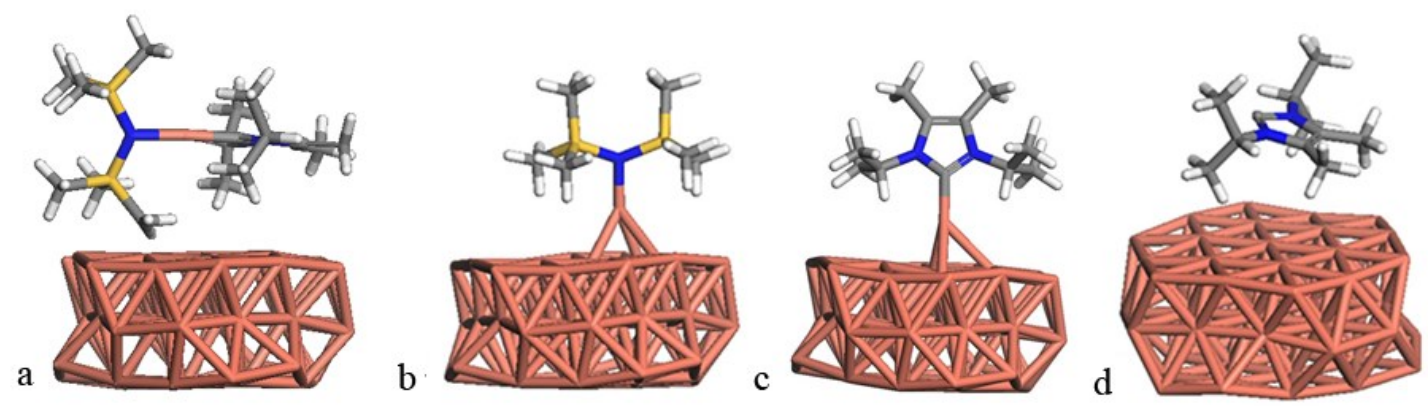

Figure 6.6 Optimized geometries of (a) 2 molecularly physisorbed on a smooth $\mathrm{Cu}$ (111) surface; (b) $\mathrm{CuN}\left(\mathrm{SiMe}_{3}\right)_{2}$ chemisorbed on the smooth surface; (c) $\mathrm{NHC}$ from 2 chemisorbed onto a rough surface; (d) NHC from 2 physisorbed on the smooth surface.

The precursors were brought to a model copper surface and relaxed with DFT in order to determine their adsorption energy $\left(\Delta E_{a d}\right)$. In both cases, the $\mathrm{Cu}$ atom of the precursor optimized to a distance of $5.4 \AA$ from the nearest surface $\mathrm{Cu}$ atom (Figure 6.6). The precursor contacted the surface via the alkyl substituent of the $\mathrm{N}\left(\mathrm{SiMe}_{3}\right)_{2}$, with a shortest $\mathrm{Cu}-\mathrm{H}$ distance of $2.4 \AA$ to the nearest surface $\mathrm{Cu}$ atom. These are non-bonded distances, indicative of molecular physisorption. We can see from the DFT energetics (Table 6.2) that the precursors have weak molecular adsorption to the surface for both $\mathbf{1}$ and 2, although there is some uncertainty in this value because of the poor description of physisorption by DFT. Explicit inclusion of van der Waals interactions in the DFT functional may give a more accurate value ${ }^{36,37}$, but the use of such functionals for organometallic reagents adsorbed onto metal surfaces is not yet well established and hence is beyond the scope of our study. ${ }^{38}$ 
It appears that steric hindrance in the ${ }^{-} \mathrm{N}\left(\mathrm{SiMe}_{3}\right)_{2}$ moiety prevents the coordinatively unsaturated $\mathrm{Cu}, \mathrm{C}$ and $\mathrm{N}$ atoms of the precursor from coming close to the surface (Figure 6.6). The only pathway to chemisorption is therefore through dissociation of the precursor at the $\mathrm{Cu}-\mathrm{NHC}$ bond. The adsorption energies show that the carbene of $\mathbf{1}$ and $\mathbf{2}$ can adsorb to the smooth surface with comparable energies (Table 6.2).

Table 6.2 Computed Adsorption Energy $\Delta E_{\text {ad }}(\mathrm{kJ} / \mathrm{mol})$ of the Precursors and Their Probable by-Products on Two Surface Models - $\mathrm{Cu}_{55}$ with a Smooth (111) Surface and $\mathrm{Cu}_{56}$ with One Extra Atom Making the Surface Rough. ${ }^{\mathrm{a}}$

\begin{tabular}{lcccc}
\multicolumn{1}{c}{ adsorbate } & surface model & structure & compound $\mathbf{1}$ & compound 2 \\
\hline $\mathrm{Cu}(\mathrm{NHC})\left[\mathrm{N}\left(\mathrm{SiMe}_{3}\right)_{2}\right]$ & smooth $\mathrm{Cu}_{55}$ & Fig 6.6a & $(-174)$ & $(-174)$ \\
$\mathrm{NHC}$ & smooth $\mathrm{Cu}_{55}$ & Fig 6.6d & $(-61)$ & $(-83)$ \\
$\mathrm{NHC}$ & rough $\mathrm{Cu}_{56}$ & Fig 6.6c & -255 & -256 \\
$\mathrm{NHC}($ dimer $)$ & smooth $\mathrm{Cu}_{55}$ & & $(-113)$ & $(-101)$ \\
$\mathrm{Cu}(\mathrm{I})(\mathrm{NHC})^{+}$ & smooth $\mathrm{Cu}_{55}$ & Fig 6.6c & -364 & -353 \\
{$\left[\mathrm{~N}\left(\mathrm{SiMe}_{3}\right)_{2}\right]^{-}$} & smooth $\mathrm{Cu}_{55}$ & & & -378 \\
{$\left[\mathrm{~N}\left(\mathrm{SiMe}_{3}\right)_{2}\right]^{-}$} & rough $\mathrm{Cu}_{56}$ & Fig 6.6b & & -385 \\
$\mathrm{Cu}(\mathrm{I})\left[\mathrm{N}\left(\mathrm{SiMe}_{3}\right)_{2}\right]$ & smooth $\mathrm{Cu}_{55}$ & Fig 6.6b & \multicolumn{3}{c}{-308} \\
\hline
\end{tabular}

${ }^{a}$ Values for physisorption are in brackets because of the limited accuracy of the PBE functional in describing van der Waals interactions.

For a smooth copper surface, the adsorption energies are low and - consistent with this the computed distances indicate that no chemical bonds are formed to the $\mathrm{Cu}$ surface. However, when a rough surface (modeled by an additional copper(I) adatom) is considered, the NHC ligands bind to this surface site with adsorption energies that are comparable to those of the $\mathrm{Cu}-\mathrm{N}\left(\mathrm{SiMe}_{3}\right)_{2}$ moiety. The DFT data do not show any significant difference between the saturated carbene (from 1) and unsaturated carbene (from 2) in terms of energetics of dissociation or adsorption during the $\mathrm{Cu}$ pulse. This 
indicates that differences between the ALD chemistry of these two precursors arise after the $\mathrm{Cu}$ pulse, probably during the plasma $\mathrm{H}_{2}$ pulse. Further studies are under way to determine the differences in the surface chemistry of these two compounds.

\subsection{Conclusion}

Two novel NHC-containing compounds 1,3-diisopropyl-imidazolin-2-ylidene copper hexamethyldisilazide (1) and 4,5-dimethyl-1,3-diisopropyl-imidazol-2-ylidene copper hexamethyldisilazide (2) were synthesized and structurally characterized. Compound $\mathbf{1}$ was found to have excellent volatility and thermal stability over an extended period of time. It was successfully employed as an ALD precursor using hydrogen plasma as a reducing agent. The deposited films had a growth rate of 0.2 $\AA /$ cycle and were crystalline. The films showed good resistivity.

Compound $\mathbf{2}$ showed excellent volatility but poorer thermal stability than $\mathbf{1}$. Under similar conditions to the ALD of $\mathbf{1}$, it did not afford a copper metal film. DFT studies of both precursors showed good dissociative chemisorption to a copper surface, with cleavage of the precursor at the carbene-copper bond, indicating similar surface chemistry during the $\mathrm{Cu}$ precursor pulse in ALD.

\subsection{Experimental}

General Considerations: All manipulations involving the synthesis and handling of copper(I) compounds were performed in an MBraun LabmasterTM 130 Dry box (mBraun, Stratham, NH, U.S.A.) under a nitrogen atmosphere. NMR spectra were recorded on a $400 \mathrm{MHz}$ Bruker AMX spectrometer. NMR spectra were measured in $\mathrm{C}_{6} \mathrm{D}_{6}$ and were referenced against residual protonated solvent. 1,3-diisopropyl-imidazolin-2- 
ylidene copper chloride was prepared from a literature method substituting imidazolinium tetrafluoroborate for imidazolinium chloride. ${ }^{39}$ 4,5-dimethyl-1,3-diisopropyl-imidazol-2ylidene copper chloride was prepared by following established literature methods for $\mathrm{NHC} \mathrm{CuCl} .^{40} 4,5$-dimethyl-1,3-diisopropyl-imidazol-2-ylidene ${ }^{41}$ and 1,3-diisopropylimidazolinium chloride ${ }^{42}$ were prepared according to literature. The diethyl ether adduct of lithium hexamethyldisilazide was prepared according to literature. ${ }^{43}$ All reagents were purchased from Sigma Aldrich (Oakville, Ontario, Canada) and used as received. All solvents were purchased as ACS grade and purified from an Mbraun Solvent Purifier System.

\section{1,3-diisopropyl-imidazolin-2-ylidene copper hexamethyldisilazide (1): 1,3-} diisopropyl-imidazolin-2-ylidene copper chloride (4.456 g, $17.52 \mathrm{mmol}$ ) was partially dissolved in $130 \mathrm{~mL}$ of toluene and cooled in the glove box freezer to $-35^{\circ} \mathrm{C}$ before use. In a separate flask, $\mathrm{Et}_{2} \mathrm{O} \cdot \mathrm{LiN}\left(\mathrm{SiMe}_{3}\right)_{2}(4.248 \mathrm{~g}, 17.6 \mathrm{mmol})$ was dissolved in $70 \mathrm{~mL}$ of toluene. The amide solution was added dropwise over the course of a hour and the cloudy solution was stirred for $24 \mathrm{~h}$, after which the solution was filtered and the insoluble material was washed with $3 \times 10 \mathrm{~mL}$ of toluene. The washings were combined with the filtrate and volatiles were stripped off under reduced pressure. The crude product was purified by sublimation $\left(\mathrm{T}_{\text {sub }}=90^{\circ} \mathrm{C}, 35 \mathrm{mTorr}\right)$ using a dry ice/acetone cold finger and collected as a colourless solid (6.322 g, 95 \%); m.p. $49-51{ }^{\circ} \mathrm{C} .{ }^{1} \mathrm{H}$ NMR $(400 \mathrm{MHz}$, $\left.\mathrm{C}_{6} \mathrm{D}_{6}\right): \delta 4.46\left[\mathrm{sept}, 2 \mathrm{H}, \mathrm{NCH}\left(\mathrm{CH}_{3}\right)_{2}\right], \delta 2.50\left[\mathrm{~s}, 4 \mathrm{H}, \mathrm{N}\left(\mathrm{CH}_{2}\right)_{2} \mathrm{~N}\right], \delta 0.81[\mathrm{~d}, 12 \mathrm{H}$, $\left.\mathrm{NCH}\left(\mathrm{CH}_{3}\right)_{2}\right], \delta 0.54\left[\mathrm{~s}, 18 \mathrm{H}, \mathrm{N}\left(\mathrm{Si}\left(\mathrm{CH}_{3}\right)_{3}\right)_{2}\right] .{ }^{13} \mathrm{C} \mathrm{NMR}\left(100 \mathrm{MHz}, \mathrm{C}_{6} \mathrm{D}_{6}\right): \delta 200.39$ $[\mathrm{NCN}], \delta 51.16\left[\mathrm{NCH}\left(\mathrm{CH}_{3}\right)_{2}\right], \delta 41.91\left[\mathrm{~N}\left(\mathrm{CH}_{2}\right)_{2} \mathrm{~N}\right], \delta 20.68[\mathrm{NCH}(\mathrm{CH} 3) 2], \delta 7.20$ [N( $\left.\left.\mathrm{Si}\left(\mathrm{CH}_{3}\right)_{3}\right)_{2}\right]$. HRMS (EI) m/z calcd for $\mathrm{C}_{15} \mathrm{H}_{36} \mathrm{~N}_{3} \mathrm{Si}_{2} \mathrm{Cu} \mathrm{M}^{+}$377.1744, found 377.1763. 


\section{4,5-dimethyl-1,3-diisopropyl-imidazol-2-ylidene copper hexamethyldisilazide (2):}

Compound $\mathbf{2}$ was prepared in an analogous manner as compound $\mathbf{1}$ substituting: 4,5dimethyl-1,3-diisopropyl-imidazol-2-ylidene copper chloride (0.518 g, $1.85 \mathrm{mmol})$ partially dissolved in $15 \mathrm{~mL}$ of toluene, $\mathrm{Et}_{2} \mathrm{O} \cdot \mathrm{LiN}\left(\mathrm{SiMe}_{3}\right)_{2}(0.449 \mathrm{~g}, 1.86 \mathrm{mmol})$ dissolved in $8 \mathrm{~mL}$ of toluene. Compound 2 was isolated by sublimation $\left(\mathrm{T}_{\text {sub }}=95^{\circ} \mathrm{C}, 25\right.$ mTorr) as a white solid $(0.630 \mathrm{~g}, 84.0 \%)$; m.p. $119{ }^{\circ} \mathrm{C} .{ }^{1} \mathrm{H}$ NMR $\left(400 \mathrm{MHz}, \mathrm{C}_{6} \mathrm{D}_{6}\right): \delta$ 4.08 [sept, 2H, $\mathrm{NCH}\left(\mathrm{CH}_{3}\right)_{2}$ ], $\delta 1.41\left[\mathrm{~s}, 6 \mathrm{H}, \mathrm{N}\left(\mathrm{CCH}_{3}\right)_{2} \mathrm{~N}\right], \delta 1.37$ [d, $\left.12 \mathrm{H}, \mathrm{NCH}\left(\mathrm{CH}_{3}\right)_{2}\right]$, $\delta 0.59\left[\mathrm{~s}, 18 \mathrm{H}, \mathrm{N}\left(\mathrm{Si}\left(\mathrm{CH}_{3}\right)_{3}\right)_{2}\right] .{ }^{13} \mathrm{C} \mathrm{NMR}\left(100 \mathrm{MHz}, \mathrm{C}_{6} \mathrm{D}_{6}\right): \delta 173.21[\mathrm{NCN}], \delta 123.08$ $\left[\mathrm{N}\left(\mathrm{CCH}_{3}\right)_{2} \mathrm{~N}\right], \delta 50.67\left[\mathrm{NCH}\left(\mathrm{CH}_{3}\right)_{2}\right], \delta 24.02\left[\mathrm{NCH}\left(\mathrm{CH}_{3}\right)_{2}\right], \delta 8.80\left[\mathrm{~N}\left(\mathrm{CCH}_{3}\right)_{2} \mathrm{~N}\right], \delta$ 7.12[N( $\left.\left.\mathrm{Si}\left(\mathrm{CH}_{3}\right)_{3}\right)_{2}\right]$. HRMS (EI) $\mathrm{m} / \mathrm{z}$ calcd for $\mathrm{C}_{17} \mathrm{H}_{38} \mathrm{~N}_{3} \mathrm{Si}_{2} \mathrm{Cu} \mathrm{M}^{+} 403.1900$, found 403.1924 .

Computational Study: The ground state electronic wave function of each molecule was calculated self-consistently within Kohn-Sham DFT using the TURBOMOLE suite of quantum chemical programs. ${ }^{44,45}$ The Perdew-Burke-Ernzerhof functiona ${ }^{46}{ }^{4}$ with the resolution-of-the-identity approximation ${ }^{47,48}$ and an all-electron valence double-zeta with polarization def-SV(P) basis set ${ }^{49}$ was considered the most suitable level of calculation. No basis set superposition error correction was required for a basis of this size. An even larger def-TZV $(\mathrm{P})^{47}$ basis set gives reaction energies that agree to less than $10 \%$, but are an order of magnitude more costly in computational time. Structures are relaxed using DFT with no constraints, except to stop adsorbates drifting towards the edge of the cluster. All the neutral $\mathrm{Cu}(\mathrm{I})$ carbene precursor molecules are closed shell compounds. A 55 copper atom cluster of $\mathrm{C}_{3 \mathrm{v}}$ symmetry, which is in the shape of a coin $\left(\mathrm{Cu}_{55}\right)$, is used as a model of the (llll 111$)$ surface to see the effect of adsorption of 
various copper precursors. A rough surface has been generated by the addition of one $\mathrm{Cu}$ atom $\left(\mathrm{Cu}_{56}\right)$. The copper coin is an open shell system with the HOMO LUMO energy difference of $<2 \mathrm{~kJ} / \mathrm{mol}$. NPA ${ }^{50}$ has been carried out in order to obtain a qualitative understanding of the charge distribution .

Crystallography: X-ray structural analysis: Crystals were selected and mounted on plastic mesh using viscous oil flash-cooled to the data collection temperature. Data were collected on a Brüker-AXS APEX CCD diffractometer with graphitemonochromated Mo-K $\alpha$ radiation $(\lambda=0.71073 \AA)$. Unit cell parameters were obtained from 60 data frames, $0.3^{\circ} \omega$, from three different sections of the Ewald sphere. The systematic absences in the data and the unit cell parameters were uniquely consistent to $P 21_{1} / n$. The data-sets were treated with absorption corrections based on redundant multiscan data. The structures were solved using direct methods and refined with fullmatrix, least-squares procedures on $F^{2}$. In $\mathbf{1}$, one isopropyl and both trimethylsilyl groups, and in 2, one trimethylsilyl group, were rotationally disordered with 1(isopropyl:trimethyl:tri-methyl):2(isopropyl)::88/12:63/37:60/40:51/49 refined site occupancies. Chemically equivalent bond distances and angles in the disordered group were constrained to average values and with equal atomic displacement atomic parameter restraints on equivalent atoms. All atoms were treated in $\mathbf{1}$ were treated with rigid bond restraints. All non-hydrogen atoms were refined with anisotropic displacement parameters. All hydrogen atoms were treated as idealized contributions. Atomic scattering factors are contained in the SHELXTL 6.12 program library. ${ }^{51}$

PE ALD: Copper thin films were deposited on silicon (100) substrates in a Beneq TFS200 ALD-reactor, capable of depositing on $200 \mathrm{~mm}$ wafers, with a remote plasma 
configuration. ${ }^{14 \mathrm{~b}}$ Plasma was generated with capacitive coupling with a $13.56 \mathrm{MHz}$ rf power source. The plasma power was $170 \mathrm{~W}$. The distance between the substrate and the grid, which was the bottom electrode, was $4 \mathrm{~cm}$. Plasma activated hydrogen was used as the reducing agent. Hydrogen gas $(99.999 \%$, AGA) was mixed with argon $(99.999 \%$, AGA) to ensure plasma ignition. In general, the hydrogen flow was $20 \mathrm{sccm}$ and the argon plasma gas flow $140 \mathrm{sccm}$. The hydrogen flow was not pulsed because no reaction between the precursor and molecular hydrogen was noticed at the applied growth temperatures. Argon carrier gas flow was typically $330 \mathrm{sccm}$. Gases were purified onsite before mixing with Aeronex GateKeeper and Entergris GateKeeper purifiers.

\subsection{References}

1 International Technology Roadmap for Semiconductors; 2011; http://www.itrs.net/.

2 (a) Chen, T. Y.; Omnes, L.; Vaisserman, J.; Doppelt, P. Inorg. Chim. Acta. 2004, 357, 1299, (b) Bollmann, D.; Merkel, R.; Klumpp, A. Microelectron. Eng. 1997, 378, 105, (c) Lagalante, A. F.; Hansen, B. N.; Bruno, T. J.; Sievers, R. E. Inorg. Chem. 1995, 34, 5781 (d) Wenzel, T. J.; Williams, E. J.; Haltiwanger, R. C.; Sievers, R. E. Polyhedron 1985, 4, 369.

3 Park, K. H.; Marshall, W. J. J. Am. Chem. Soc. 2005, 127, 9330.

4 (a)Li, Z. W.; Rahtu, A.; Gordon, R. G. J. Electrochem. Soc. 2006, 153, C787, (b) Li, Z. W.; Barry, S. T.; Gordon, R. G. Inorg. Chem. 2005, 44, 1728, (c) Lim, B. S.; 
Rahtu, A.;Gordon, R. G. Nat. Mater. 2003, 2, 749, (d) Lim, B. S.; Rahtu, A.; Park, J. S.; Gordon, R. G. Inorg. Chem. 2003, 42, 7951.

5 Coyle, J. P.; Monillas, W. H.; Yap, G. P. A.; Barry, S. T. Inorg. Chem. 2008, 47, 683.

6 Park, J. W.; Jang, H. S.; Kim, M.; Sung, K.; Lee, S. S.; Chung, T.; Koo, S.; Kim, C. G.; Kim, Y. Inorg. Chem. Commun. 2004, 7, 463.

7 Grushin, V. V.; Marshall, W. J. Adv. Synth. Catal. 2004, 346, 1457.

8 Knoops, H. C. M.; Langereisb, E.; van de Sandenb, M. C. M.; Kessels, W. M. M J. Electrochem. Soc. 2010, 157, 12, G241.

9 Gambarotta, S.; Bracci, M.; Floriani, C.; Chiesi-Villa, A.; Guastini, C. J. Chem. Soc., Dalton Trans. 1987, 1883.

10 Tsuda, T.; Watanabe, K.; Miyata, K.; Yamamoto, H.; Saegusa, T. Inorg. Chem. 1981, 20, 2728.

11 Gaillard, S.; Cazin, C. J.; Nolan, S. P. Acc. Chem. Res. 2012, 45, 778, and references therein.

12 (a) Goj, L. A.; Blue, E. D.; Delp, S. A.; Gunnoe, T. B.; Cundar, T. R.; Pierpont, A. W.; Petersen, J. L.; Boyle, P. D. Inorg. Chem. 2006, 45, 9032, (b) Goj, L. A.; Blue, E. D.; Munro-Leighton, C.; Gunnoe, T. B.; Petersen , J. L. Inorg. Chem. 2005, 44, 8647.

13 Mankad, N. P.; Laitar, D. S.; Sadighi, J. P. Organometallics 2004, 23, 3369.

14 (a) Niskanen, A.; Hatanpää, T.; Arstila, K.; Leskelä, M.; Ritala, M. Chem. Vap. Deposition 2007, 13, 408, (b) Kariniemi, M.; Niinistö, J.; Hatanpää, T.; Kemell, M.; Sajavaara, T.; Ritala, M.; Leskelä, L. Chem. Mater. 2011, 23, 2901

15 Denk, M. K.; Thadani, A.; Hantano, K.; Lough, A. J. Angew. Chem. Int. Ed. 1997, 36,2607

16 Jain, A.; Chi, K. -M.; Kodas, T. T.; Hampden-Smith, M. J. J. Electronchem. Soc. 1993, 140,1434

17 (a) Nyburg, S. C.; Parkins, A. W.; Sidi-BouMedine, M. Polyhedron 1993, 12, 1119 , (b) Melzer, M. M.; Mossin, S.; Day, X.; Bartell, A. M.; Kapoor, P.; Meyer, K.; Warren, T. H. Angew. Chem. Int. Ed. 2010, 49, 904, (c) Harkins, S. B.; Mankad, N. P.; Miller, A. J. M.; Szilagyi, R. K.; Peters, J. C. J. Am.Chem. Soc. 2008, 130, 3478. 
18 James, A. M.; Laxman, R. K.; Fronczek, F. R.; Maverick, A. W. Inorg. Chem. 1998, 37,3785

19 Elliott, S. D. Semicond. Sci. Tech. 2012, 27, 074008.

20 Widjaja, Y; Musgrave, C. B J. Chem. Phys. 2002, 117, 1931.

21 Jeloaica, L.; Esteve, A.; Rouhani, M. D.; Esteve, D. Appl. Phys. Lett. 2003, 83, 542.

22 Li, J.; Wu, J.; Zhou, C.; Han, B.; Lei, X.; Gordon, R.; Cheng, H. Int. J. Quantum Chem. 2009, 109, 756.

23 Kwon, J.; Saly, M.; Halls, M. D.; Kanjolia, R. K.; Chabal, Y. J. Chem. Mater. 2012, $24,1025$.

24 (a) Mårtensson, P.; Larsson, K.; Carlsson, J.-O. Appl. Surf. Sci. 1998, 136, 137, (b) Mårtensson, P.; Larsson, K.; Carlsson, J.-O. Applied Surface Science 1999, 148, 9, (c) Mårtensson, P.; Larsson, K.; Carlsson, J.-O. Appl. Surf. Sci. 2000, 157, 92.

25 Dai, M.; Kwon, J.; Halls, M. D.; Gordon, R. G.; Chabal, Y. J. Langmuir 2010, 26, 3911.

26 Orimoto, Y.; Toyota, A.; Furuya, T.; Nakamura, H.; Uehara, M.; Yamashita, K.; Maeda, H. Ind. Eng. Chem. Res. 2009, 48, 3389.

27 Machado, E.; Kaczmarski, M.; Ordejón, P.; Garg, D.; Norman, J.; Cheng, H. Langmuir 2005, 21, 7608.

28 Dey, G.; Elliott, S. D. J. Phys. Chem. A 2012, 116, 8893.

29 Lee, B. H.; Hwang, J. K.; Nam, J. W.; Lee, S. U.; Kim, J. T.; Koo, S.-M.; Baunemann, A.; Fischer, R. A.; Sung, M. M. Angew. Chem. Int. Ed. 2009, 48, 4536.

30 a) Lin, J. C. Y.; Huang, R. T. W.; Lee, C. S.; Bhattacharyya, A.; Hwang, W. S.; Lin, I. J. B. Chem. Rev. 2009, 109, 3561, b) An, D.; Wang, J.; Dong, T.; Yang, Y.; Wen, T.; Zhu, H.; Lu, X.; Wang, Y. Eur. J. Inorg. Chem. 2010, 4506

31 Kunte, G. V.; Shivashankar, S. A.; Umarji, A. M. Meas. Sci. Technol. 2008, 19, 025704 .

32 The 1 Torr values for $\mathbf{1}$ and $\mathbf{2}$ are corrected from our previous publication (Coyle et al. Chem. Mater. 2013, 25, 1132) because of a calculation error for the value of the sample pan surface area. 
33 Park, K.-M.; Kim, J.-K.; Han, B.; Lee, W.-J.; Kim, J.; Shin, H.-K. Microelectron. Eng. 2012, 89, 27.

34 Niskanen, A.; Rahtu, A; Sajavaara, T.; Arstila, K.; Ritala, M.; Leskela, M. J. Electrochem. Soc. 2005, 152, G25.

35 Li, Z.; Rahtu, A.; Gordon, R. G. J. Electrochem Soc. 2006, 153, C787

36 Lundqvist, B. I.; Andersson, Y.; Shao, H.; Chan, S.; Langreth, D. C. Int. J. Quantum Chem. 1995, 56, 247.

37 Ruiz, V. G.; Liu, W.; Zojer, E.; Scheffler, M.; Tkatchenko, A. Phys. Rev. Lett. 2012, $108,146103$.

38 Marom, N.; Tkatchenko, A.; Rossi, M.; Gobre, V. V.; Hod, O.; Scheffler, M.; Kronik, L. J. Chem. Theory Comput. 2011, 7, 3944.

39 Dubinina, G.G.; Furutachi, H.; Vicic, D. A. J. Am. Chem. Soc. 2008, 130, 8600.

40 Díez-Gonzáez, S.; Escudero-Adán, E. C.; Benet-Buchholz, J.; Stevens, E. D.; Slawin, A. M. Z.; Nolan, S. P. Dalt. Trans. 2010, 39, 7595.

41 Kuhn, N.; Kratz, T. Synthesis 1993, 561.

42 Bittermann, A.; Baskakov, D.; Herrmann, W. A. Organometallics 2009, 28, 5107.

43 Lappert, M. F.; Slade, M. J.; Singh A.; Atwood, J. L.; Rogers, R. D.; Shakir, R. J. Am. Chem. Soc. 1983, 105, 302.

44 Schafer, A.; Huber, C.; Ahlrichs, R. J. Chem. Phys. 1994, 100, 5829.

45 Ahlrichs, R.; Bär, M.; Häser, M.; Horn, H.; Kölmel, C. Chem. Phys. Lett. 1989, 162, 165.

46 Perdew, J. P.; Burke, K.; Ernzerhof, M. Phys. Rev. Lett. 1996, 77, 3865.

47 Eichkorn, K.; Weigend, F.; Treutler, O.; Ahlrichs, R. Theor. Chim. Acta. 1997, 97, 119.

48 Sierka, M.; Hogekamp, A.; Ahlrichs, R. J. Chem. Phys. 2003, 118, 9136.

49 Schafer, A.; Horn, H.; Ahlrichs, R. J. Chem. Phys. 1992, 97, 2571.

50 Cioslowski, J. J. Am. Chem. Soc. 1989, 111, 8333. 
51 Sheldrick, G.M. Acta Cryst. 2008, A64, 112-122. 


\section{Chapter: Synthesis and Thermal Gravimetric Studies of Monomeric $\mathrm{Cu}(\mathrm{I}) \mathrm{N}\left(\mathrm{SiMe}_{3}\right)_{2}$ Complexes Supported by N-Heterocyclic and Acyclic Diamino Carbenes.}

A manuscript for the following work is in preparation. Co-contributors to this work include Eric R. Sirianni ${ }^{l}$, Ilia Korobkov ${ }^{2}$, Glenn P.A. Yap ${ }^{1}$, Seán T. Barry ${ }^{3}$.

${ }^{1}$ Department of Chemistry \& Biochemistry, University of Delaware, Newark, Delaware 19716, United States

${ }^{2}$ Department of Chemistry, University of Ottawa, Ottawa, K1N 6N5, Canada

${ }^{3}$ Department of Chemistry, Carleton University, 1125 Colonel By Drive, Ottawa, K1S 5B6, Canada

\subsection{Abstract}

Thermal properties of a series of monomeric copper(I) hexamethyldisilazide complexes supported by N-heterocyclic and acyclic diamino carbenes were evaluated for consideration as ALD precursors. Ten NHCs and ADCs were selected to study the impact of $\mathrm{N}$-alkyl substituent and backbone character on volatility and thermal stability. The series of complexes had melting points of $45-184{ }^{\circ} \mathrm{C}$ while several complexes were liquids. Vaporizations rates were measured by stepped-isothermal TGA experiments between $110-170{ }^{\circ} \mathrm{C}$. Enthalpies of vaporization were determined to be $63-90 \mathrm{~kJ} / \mathrm{mol}$. Temperatures for 1 Torr of vapor pressure were estimated to be $143-172{ }^{\circ} \mathrm{C}$ showing a general dependence on molecular weight. The imidazolylidene complexes were thermally unstable with strong evidence indicating the unsaturated backbone as a point of weakness. The imidazolinylidene complexes showed excellent thermal stability with comparable results for the formamidinylidenes complexes. The steric parameter of the

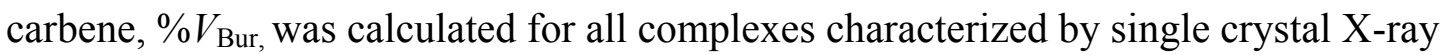
diffraction. NHCs and ADCs are suitable additions to the library of ligands available to precursor design for ALD. 


\subsection{Introduction}

Copper precursors for atomic layer deposition (ALD) and chemical vapour deposition (CVD) remain a subject of research internationally due to the importance of copper as an interconnect material in microelectronics. ${ }^{1}$ Copper is easily reduced to a metallic film, and this has led to an abundance of copper precursors, including $\beta$ diketonates, ${ }^{2} \beta$-diketiminates, ${ }^{3}$ amidinates, ${ }^{4}$ guanidinates, ${ }^{5}$ aminoalkoxides, ${ }^{6}$ pyrrolylaldiminates, ${ }^{7}$ and disilylamides ${ }^{8}$ to name a few.

Three main criteria outline the design of a copper precursor capable of meeting the requirements for industrial application. These criteria centre on the precursor's volatility, thin film deposition chemistry, and chemical composition. The first two criteria are temperature dependent preferably met at low temperatures to reduce potential issues related to thermal stability and thin film morphology. The precursor needs to generate sufficient vapour pressure at a temperature where negligible decomposition or molecular rearrangement occurs. Precursor chemisorption and reactivity of the surface species to copper metal at appreciable surface coverages will enable an acceptable growth rate. Lastly, the precursor molecule must not contain atoms that contaminate or degrade the barrier and adhesion layers, increase the resistance of the copper interconnect, or compromise the longevity of the microelectronic devices.

Recently, our efforts have been focused on N-heterocyclic carbenes (NHC) supported copper(I) amide compounds as volatile precursors for ALD. The typical copper(I) amide is a tetramer, ${ }^{9}$ but 1,3-diisopropyl-imidazolin-2-ylidene copper hexamethyldisilazide (HMDS) was successful employed as a plasma enhanced atomic layer deposition precursor. ${ }^{1}$ This compound was shown to have good volatility and 
excellent thermal stability over a long time and temperature range. A growth rate for copper metal at $225^{\circ} \mathrm{C}$ of $0.2 \AA /$ cycle was achieved. Interestingly, the similar compound 4,5-dimethyl-1,3-diisopropyl-imidazol-2-ylidene copper HMDS was found to leave a high residual mass in thermal gravimetric analysis. The main structural difference between these two compounds was the existence of a double bond in the backbone of the carbene moiety (Figure 7.1).
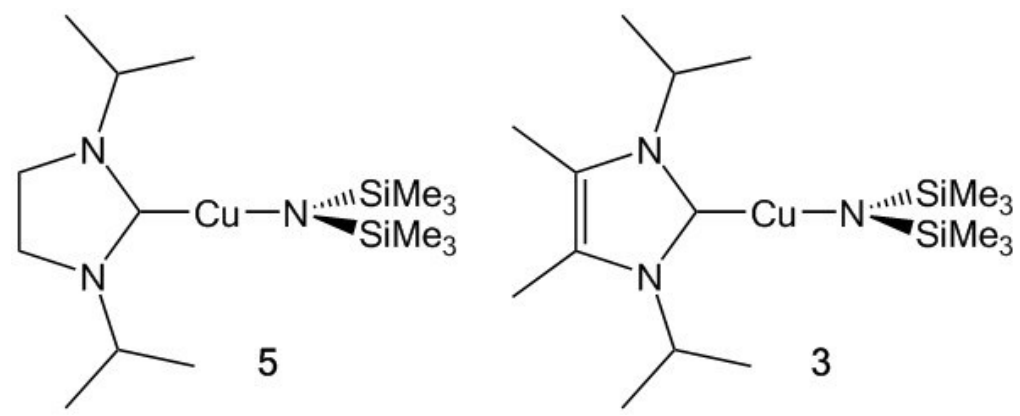

Figure 7.1 Structural differences between 1,3-diisopropyl-imidazolin-2-ylidene copper hexamethyldisilazide (5) and 4,5-dimethyl-1,3-diisopropyl-imidazol-2-ylidene copper hexamethyldisilazide (3).

As a concept for a copper precursor, the formulation of a NHC copper(I) amide matches well with the criteria mentioned above. The tendency of NHCs to form linear, monomeric complexes with copper ensures a low molecular mass to aid volatility. The high basicity of a metal amide bond can engender surface species amenable to reaction with reducing agents. Common contaminates, such as halides and oxygen, are avoided. The variable structural features of NHCs (N-substituents, backbone, and ring size) allows for tuneable physical characteristics such as thermal stability and volatility. Although, the strong copper-NHC bond may present a potential drawback as it could hamper precursor chemisorption. ${ }^{8,10}$

N-heterocyclic carbenes have risen to become workhorse ligands in organometallic catalysis. ${ }^{11}$ Research efforts have mainly focused on the stereo-electronic 
effects of the metal-NHC bond to tune catalyst performance with high turnover rates for a multitude of transformations. ${ }^{12}$ Decomposition routes of NHC metal complexes ${ }^{13}$ are also important to understand in order to circumvent catalyst deactivation. ${ }^{14}$ Copper NHC complexes are extremely well studied ${ }^{15}$ and exploited in catalysis. ${ }^{16}$ The copper-NHC bond is very strong $(\sim 290 \mathrm{~kJ} / \mathrm{mol})^{8,17}$ and these catalysts have not shown a high susceptibility to decomposition; therefore, screening of copper NHC catalyst systems have been performed to optimize catalytic activity ${ }^{18}$ and not stability.

This encouraged us to explore the effect of the structure of the carbene on the principle precursor characteristics of these types of compounds. In general, a wide variety of NHCs can be synthesized. ${ }^{19}$ We undertook to study the effects of steric bulk on the nitrogen atoms and at the backbone carbons, as well as the bond order of the carboncarbon backbone bond. From the excellent work of Alder et al., ${ }^{20}$ acyclic diamino carbenes (ADC) are also accessible, and this broadened the scope of this study.

Herein we report a series of heterocyclic and acyclic carbenes supporting copper(I) HMDS. The synthesis, structures, and thermal chemistries are compared across this family of copper compounds to highlight their potential utility as vapour precursors for CVD and ALD.

\subsection{Results and Discussion}

A straightforward approach was used to synthesize the NHC and ADC copper(I) HMDS complexes. The target complexes were generated by adding 2 equivalents of lithiated HMDS to solutions of the respective carbene salt and copper chloride in THF (Figure 7.2). ${ }^{21}$ Non-nucleophilic lithium amides are suitable bases to generate free carbenes $^{19,20 \mathrm{a}}$ and prior isolation of the carbene copper chlorides was unnecessary to 
achieve satisfactory yields. Most complexes were sublimed or distilled above $75{ }^{\circ} \mathrm{C}$ under reduced pressure ( $<35$ mTorr) in fair to very good yields, except for $\mathbf{1}$ which decomposed when heated. The procedure for obtaining compound $\mathbf{1 0}$ was slightly modified due to a mixture of counterions in the formamidinium salt. ${ }^{20 \mathrm{c}}$ These colourless compounds quickly turned brown when exposed to air. There are some reports of NHCcopper complexes reacting readily with $\mathrm{O}_{2}$ to produce the corresponding ketone ${ }^{22}$ while free NHCs are sensitive towards $\mathrm{H}_{2} \mathrm{O} .{ }^{22 b}$ Additionally, $\left[\mathrm{CuN}\left(\mathrm{SiMe}_{3}\right)_{2}\right]_{4}$ decomposes slowly in air. ${ }^{23}$ Compounds $\mathbf{8}-\mathbf{1 0}$ are the first reported examples of ADC copper complexes.

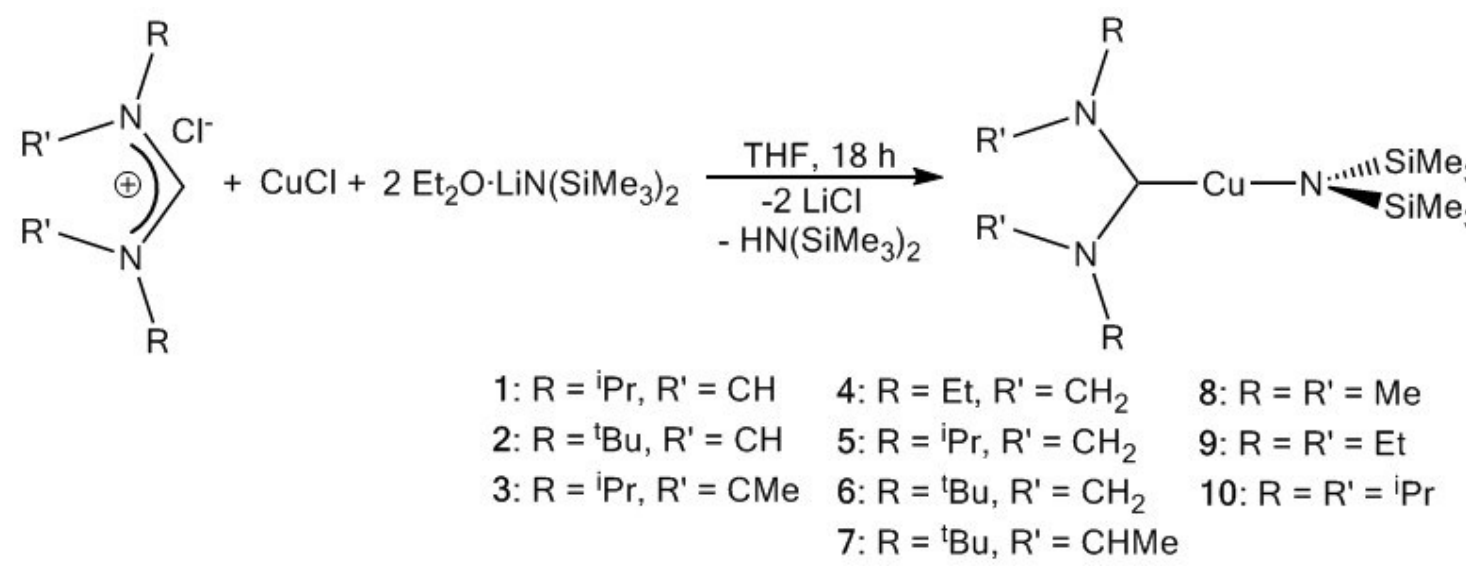

Figure 7.2 General Synthetic Conditions for 1-10.

All imidazolium, imidazolinium, formamidinium chlorides were prepared from known literature methods with the exception of 4,5-dimethyl-1,3-ditertbutylimidazolinium chloride. This new imidazolinium was developed from known synthetic methods (Figure 7.3). Briefly, a condensation reaction between formaldehyde and 


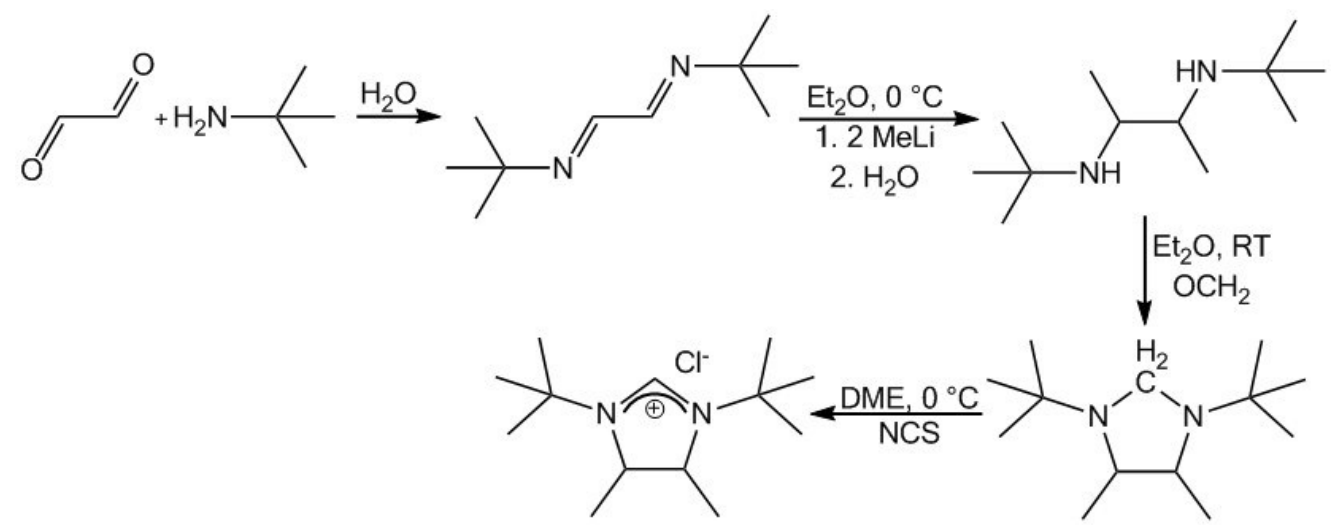

Figure 7.3 Synthesis of 4,5-dimethyl-1,3-ditertbutyl-imidazolinium chloride.

rac-N,N'-di-tert-butyl-2,3-dimethylethylenediamine ${ }^{24}$ generated the corresponding aminal which was oxidized by $\mathrm{N}$-chlorosuccinimide. ${ }^{25}$ Attempts involving the traditional orthoformate route failed presumably due to steric crowding preventing imidazolinium salt formation.

Table 7.1 Properties of Diamino Carbene Cu(I) HMDS Compounds $\mathbf{1}-\mathbf{1 0 .}$

\begin{tabular}{ccccccccc}
\hline Compound & TGA & ${ }^{13} \mathrm{C}_{\mathrm{NHC}}$ & ${ }^{13} \mathrm{C}_{\mathrm{NHC}}$ & $\Delta^{13} \mathrm{C}$ & $\% V_{\text {bur }}{ }^{\mathrm{c}}$ & $\mathrm{Mp}$ & 1 Torr & $\Delta \mathrm{H}_{\text {vap }}$ \\
\hline $\mathbf{1}$ & - & 210.5 & 176.9 & 33.6 & - & - & - & - \\
$\mathbf{2}$ & 14.7 & 213.2 & 177.0 & 36.2 & 41.5 & 119 & - & - \\
$\mathbf{3}$ & 4.4 & 206.8 & 173.2 & 33.6 & 40 & 115 & 158 & 71.3 \\
$\mathbf{4}$ & 1.5 & 237.7 & 201.3 & 36.4 & - & liquid & 153 & 66.1 \\
$\mathbf{5}$ & 1.1 & 236.8 & 200.4 & 36.4 & 35.7 & 51 & 149 & 66.5 \\
$\mathbf{6}$ & 0.7 & 238.2 & 201.5 & 36.7 & - & 98 & 172 & 63.0 \\
$\mathbf{7}$ & 1.0 & 238.6 & 198.3 & 40.3 & 42.3 & 118 & 162 & 66.4 \\
$\mathbf{8}$ & 1.6 & 259.7 & 214.1 & 45.6 & 30.7 & 45 & 143 & 65.5 \\
$\mathbf{9}$ & 0.6 & 252.0 & 211.4 & 40.6 & - & liquid & 145 & 70.5 \\
$\mathbf{1 0}$ & 1.1 & 255.5 & 221.0 & 34.6 & 45.6 & 184 & 169 & 89.6 \\
\hline
\end{tabular}

${ }^{\text {a }} \mathrm{TG}$ experiments were preformed with a heating rate of $10^{\circ} \mathrm{C} / \mathrm{min}$. Sample masses were $1.3 \mu \mathrm{mol}(4-6 \mathrm{mg})$. Residual mass recorded at $350^{\circ} \mathrm{C}$.

${ }^{b} \mathrm{C}$ chemical shifts for $\mathrm{C} 2$ of the free diamino carbenes were taken from Ref. 28(a) except for 7 which is reported here. ${ }^{26}$

${ }^{\mathrm{c}} \% V_{\mathrm{Bur}}$ calculated with scaled (by 1.17 ) Bondi radii, sphere radius $\mathrm{R}=3.5 \AA$, and diamino carbene - metal bond length of $2.00 \AA$. 
${ }^{1} \mathrm{H}$ NMR analysis of 1-10 displayed a single set of peaks consistent with the expectation for a single solution species. The singlet peak for HMDS in $\mathbf{1}-\mathbf{1 0}$ was found between $0.54-0.62 \mathrm{ppm}$. In $\left[\mathrm{CuN}\left(\mathrm{SiMe}_{3}\right)_{2}\right]_{4}$, this peak was reported at $0.505 \mathrm{ppm} .{ }^{27}$ For $\mathbf{8}$ - 10, a single set of peaks for the ADC alkyl groups were observed showing free rotation around the central $\mathrm{C}-\mathrm{N}$ bonds. ${ }^{13} \mathrm{C}$ NMR spectra were also simple to interpret. The ${ }^{13} \mathrm{C}$ carbenic $(\mathrm{C} 2)$ peaks were found within the ranges of $173-177 \mathrm{ppm}$ for the imidazolylidenes (1-3), 198- 201 ppm for the imidazolinylidenes (4-7), and 211- 221 ppm for the formamidinylidenes $(\mathbf{8}-\mathbf{1 0})$ (Table 7.1) which is consistent with further downfield shifts for the stronger $\sigma$-donor diamino carbenes. ${ }^{28}$

Interestingly, the $\mathrm{C} 2$ chemical shifts for the free imidazolylidenes in $\mathbf{1}$ - $\mathbf{3}$ were shifted upfield 34 - 36 ppm upon complexation. Similarly, an upfield shift of 36 - 40 ppm was observed for the imidazolinylidenes in $\mathbf{4}-\mathbf{7}$. The upfield shifts for the formamidinylidenes varied. Compounds $\mathbf{8}$ and $\mathbf{9}$ displayed large upfield shifts of 45.6 ppm and 40.5 ppm, respectively, while an upfield shift of only 34 ppm was observed in 10. This trend in upfield shifts coincides with the $\sigma$-donor and $\pi$-acceptor abilities of the diamino carbenes. ${ }^{29}$ Intuitively, this trend can be linked to the $\pi$-acceptor property of the carbene as $\pi$-back donation from the copper center would increase the shielding around C2. $\pi$-back bonding from group 11 metals to NHCs is no longer considered as negligible. ${ }^{17,30}$ In fact, $\pi$-back-bonding has been studied in a series of tripodal NHC group 11 complexes $^{31}$ and the effects of this phenomenon have manifested in gold catalysis. ${ }^{32}$ The outlier data point from $\mathbf{1 0}$ is possibly due to steric hindrance preventing appropriate orbital overlap for sufficient $\pi$-back-bonding to occur. A more rigorous theoretical and 
structural analysis is warranted to fully understand this apparent trend in ${ }^{13} \mathrm{C}$ chemical shifts. $^{28 \mathrm{a}}$
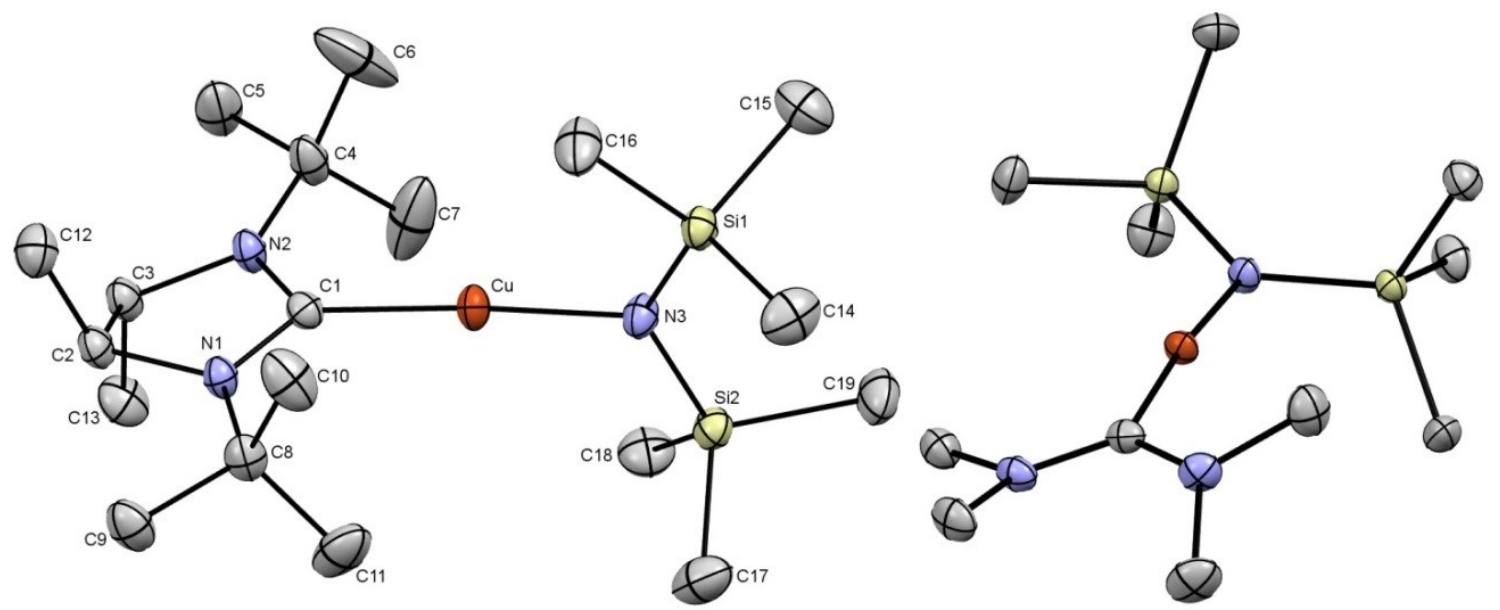

Figure 7.4 Molecular diagram of 7 (left) and 8 (right) with thermal ellipsoids at $30 \%$ probability. H-atoms omitted for clarity.

Crystals were isolated for several of the compounds $(\mathbf{2}, \mathbf{7}, \mathbf{8}$, and 10) and these structures were very similar regardless of alkyl groups and indeed regardless of whether the carbene was heterocyclic or acyclic (Figure 7.4; Table 7.2 \& 7.3 in Supporting Information). In general, the structures deviated only slightly from our previously reported copper carbene compounds (3 and 5). ${ }^{8}$

All of the compounds have a roughly linear coordination of copper $\left(\sim 174^{\circ}-\right.$ $180^{\circ}$ ), with 8 showing the highest deviation from linear. This is interesting, since $\mathbf{8}$ has the least constraint of the carbene groups, but does not show as high steric bulk as $\mathbf{1 0}$ does. This deviation also does not appear to corroborate with the torsion of the hexamethyldisilazido ligand to the carbene (i.e. $\mathrm{N}-\mathrm{C}-\mathrm{N}-\mathrm{Si}$ ), which rules out hyperconjugation through the copper as having a primary effect on the coordination geometry. Both the copper carbene bonds $(1.87 \AA-1.91 \AA)$ and the copper amide bonds $(1.84 \AA-$ $1.87 \AA$ ) are in a close range across this family of compounds. The $\mathrm{Si}-\mathrm{N}-\mathrm{Si}$ bond angles 
range between $126-131^{\circ}$. This bond angle is $117.9^{\circ}$ in $\left[\mathrm{CuN}\left(\mathrm{SiMe}_{3}\right)_{2}\right]_{4}{ }^{23}$ This difference likely indicates the amido ligand geometry is relaxed from steric pressure in a monomeric compound.

One interesting aspect of these structures is the angle between the ligand planes. In all cases, the carbenes were trigonal planar around the carbenic carbon, and in general the HMDS was trigonal planar around the nitrogen (with $\mathbf{3}$ showing the largest deviation at $356^{\circ}$ ). Most of the compounds had an angle between planes of $74^{\circ}-88^{\circ}$ (including the previously reported compound $\mathbf{3}^{8}$ ), while $\mathbf{5}$ showed only $56^{\circ}$ angle and $\mathbf{8}$ showed $23^{\circ}$.

This is obviously an effect of steric bulk (and possibly crystalline packing) since these are the two examples of lower steric bulk carbenes for heterocyclic and acyclic respectively.

Table 7.2 Selected Bond Lenghts and Bond Angles for the Structures of 2, 3, 5, 7, 8 and 10. ${ }^{\mathrm{a}}$ Data was previously reported and provided for convenience. ${ }^{8}$

\begin{tabular}{|c|c|c|c|c|c|c|}
\hline & 2 & $3^{\mathrm{a}}$ & $5^{\mathrm{a}}$ & 7 & 8 & 10 \\
\hline \multicolumn{7}{|c|}{ selected bond lengths $(\AA)$} \\
\hline $\mathrm{Cu}-\mathrm{C} 1$ & $1.894(2)$ & $1.881(2)$ & $1.870(5)$ & $1.897(2)$ & $1.905(1)$ & $1.908(1)$ \\
\hline $\mathrm{Cu}-\mathrm{N} 3$ & $1.864(1)$ & $1.870(2)$ & $1.836(4)$ & $1.870(2)$ & $1.856(1)$ & $1.873(1)$ \\
\hline $\mathrm{C} 1-\mathrm{N} 1$ & $1.369(3)$ & $1.360(3)$ & $1.330(7)$ & $1.343(3)$ & $1.352(2)$ & $1.355(1)$ \\
\hline $\mathrm{C} 1-\mathrm{N} 2$ & $1.362(2)$ & $1.365(3)$ & $1.336(6)$ & $1.354(3)$ & $1.336(2)$ & $1.355(1)$ \\
\hline N3-Si1 & $1.698(2)$ & $1.697(2)$ & $1.680(6)$ & $1.695(2)$ & $1.700(1)$ & $1.662(4)$ \\
\hline N3-Si2 & $1.705(2)$ & $1.692(2)$ & $1.701(5)$ & $1.702(2)$ & $1.704(1)$ & $1.708(4)$ \\
\hline \multicolumn{7}{|c|}{ selected bond angles (deg) } \\
\hline $\mathrm{C} 1-\mathrm{Cu}-\mathrm{N} 3$ & $179.07(7)$ & $178.59(9)$ & $179.2(2)$ & $175.8(1)$ & $173.78(5)$ & 180.0 \\
\hline $\mathrm{N} 1-\mathrm{C} 1-\mathrm{N} 2$ & $104.2(1)$ & $103.8(2)$ & $106.6(5)$ & $108.0(2)$ & $118.6(1)$ & $116.95(18$ \\
\hline Si1-N3-Si2 & $125.86(9)$ & $131.3(1)$ & $128.2(3)$ & 127.1(1) & $126.10(6)$ & $127.45(7)$ \\
\hline \multicolumn{7}{|c|}{ sum of angles (deg) } \\
\hline N1 & 360.0 & 360.0 & 360.0 & 359.3 & 359.1 & 358.2 \\
\hline N2 & 360.0 & 359.9 & 359.9 & 359.7 & 358.8 & 360.0 \\
\hline N3 & 357.6 & 355.7 & 360.0 & 359.4 & 358.6 & 360.0 \\
\hline $\mathrm{C} 1$ & 360.0 & 360.0 & 360.0 & 359.9 & 359.7 & 359.9 \\
\hline \multicolumn{7}{|c|}{ angle between planes (deg) } \\
\hline $\begin{array}{l}(\mathrm{Si} 1, \mathrm{~N} 3, \mathrm{Si} 2) \\
(\mathrm{N} 1, \mathrm{C} 1, \mathrm{~N} 2)\end{array}$ & 73.9 & 82.4 & 56.6 & 87.9 & 21.5 & 82.9 \\
\hline \multicolumn{7}{|c|}{ torsion angles (deg) } \\
\hline N1-C1-N3-Si1 & $82.5(2)$ & $88.4(2)$ & $55.9(5)$ & $85.4(2)$ & $33.4(1)$ & $80.3(9)$ \\
\hline $\mathrm{N} 1-\mathrm{C} 2-\mathrm{C} 3-\mathrm{N} 2$ & $0.2(2)$ & $0.8(3)$ & $8.5(7)$ & $28.7(2)$ & - & - \\
\hline
\end{tabular}


The percentage of buried volume $\left(\% V_{\mathrm{Bur}}\right)$ is a steric parameter model that calculates the percentage of a sphere around a metal atom occupied by a ligand..$^{33}$ This model can be used for a variety of ligands and has been used extensively in the characterisation of metal-NHC complexes. The $\% V_{\text {Bur }}$ values were calculated from experimental structure data and were between 30.7 and $45.6 \%$ (Table 7.1). $\mathrm{I}^{t} \mathrm{Bu}_{2} \mathrm{Me}_{2}$ is a new NHC with a value of $40.7 \%$. The series of NHCs in $\mathbf{2}, \mathbf{3}$, and $\mathbf{7}$ displays excellent fine tuning of the steric parameter. The $\mathrm{ADC}, \mathrm{AMe}_{4}$, had a value of $30.7 \%$ which is a higher value than some larger $\mathrm{NHCs}^{34}$ and is likely because of the difference in their core $\mathrm{N}-\mathrm{C}-\mathrm{N}$ bond angles.

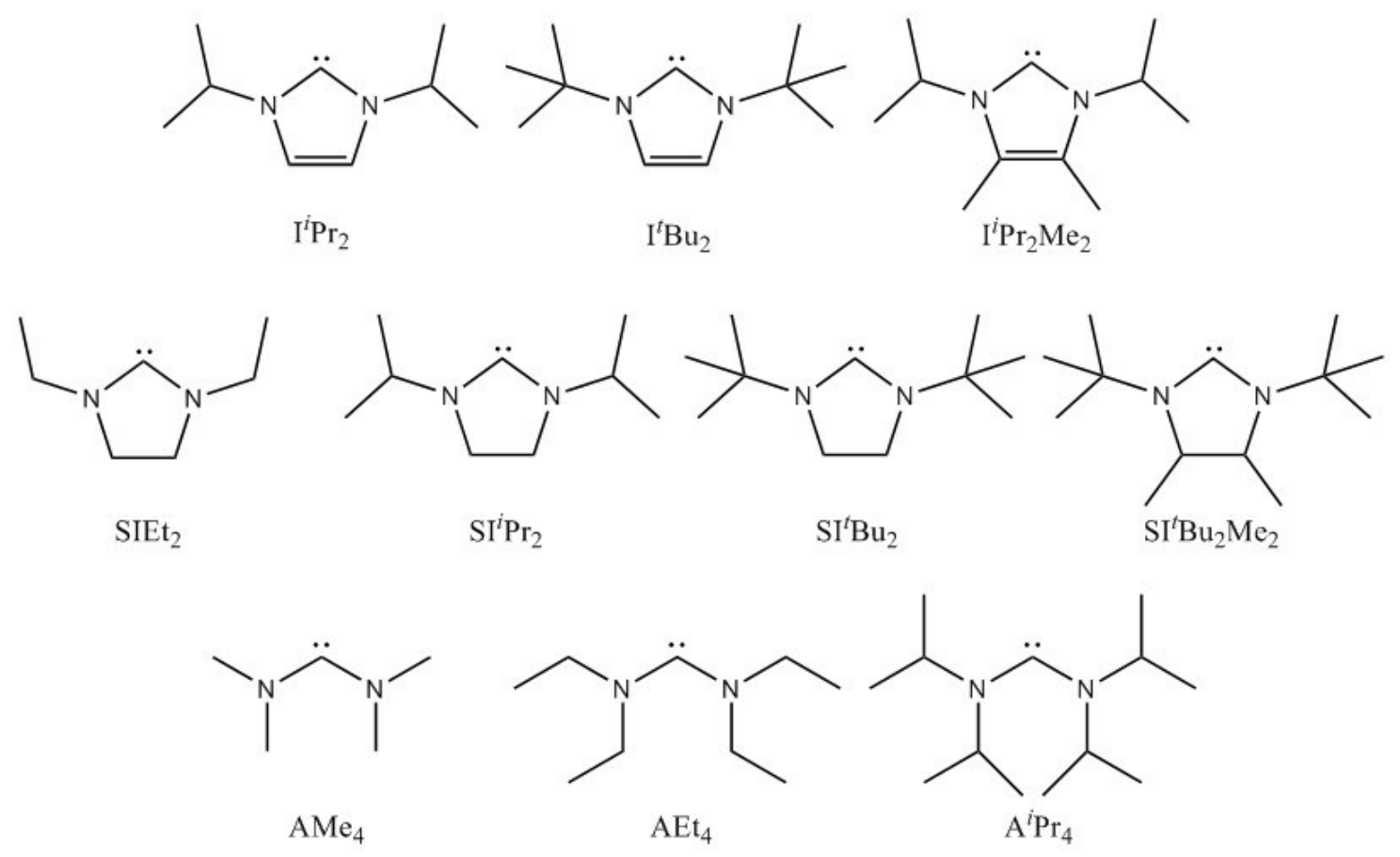

Figure 7.5 Imidazolylidenes and Imidazolinylidenes, N-heterocyclic Carbenes (NHC); and the Formamidinylidenes, Acyclic Diamino Carbenes (ADC), Evaluated in Monomeric $\mathrm{Cu}(\mathrm{I}) \mathrm{HMDS}$ Complexes 1-10.

All the carbenes employed in this study (Figure 7.5) were systematically selected concurrent with thermal gravimetric analysis (TGA) of the corresponding copper complex. The thermal stability of each complex influenced the subsequent choice of 
carbene. Our starting point was ${ }^{i} \operatorname{Pr}_{2}$ simply because imidazolylidenes and ${ }^{i} \operatorname{Pr}$ substituents are ubiquitous in their respective fields of NHCs and CVD precursors. Fittingly, $\mathbf{1}$ proved to be the least thermally stable. All attempts to purify $\mathbf{1}$ by vacuum techniques resulted in a low yield of viscous oil which contained $\mathbf{1}$ amongst decomposition products, as determined by ${ }^{1} \mathrm{H}$ NMR. Decomposition was thwarted by increasing the steric bulk of the carbene, hence 2 was easily purified in high yields by sublimation. Interestingly, TGA of 2 showed a two feature weight loss curve indicative of decomposition (Figure 7.6).

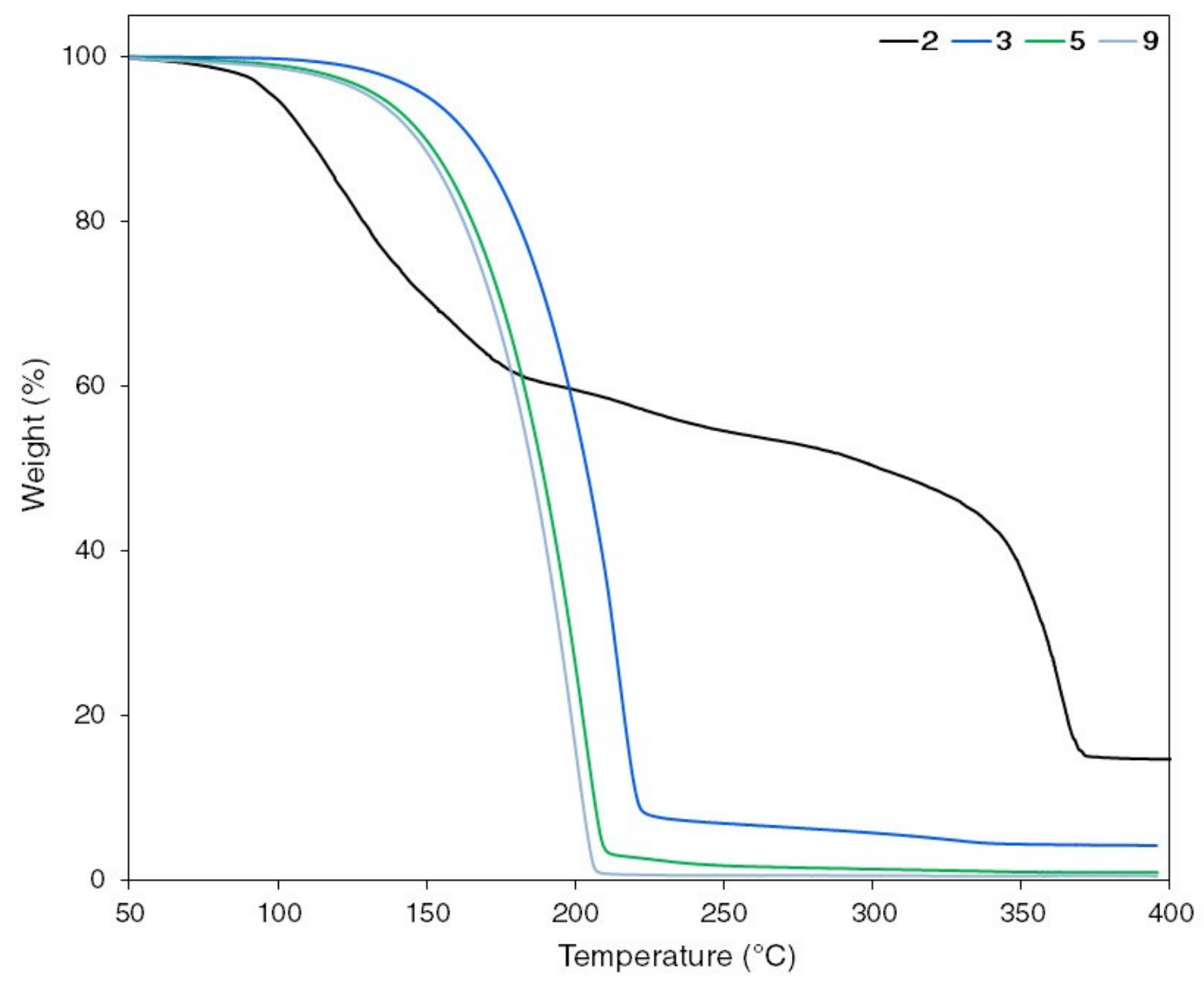

Figure 7.6 Thermal gravimetric analysis of $\mathbf{2}, \mathbf{3}, \mathbf{5}$, and $\mathbf{9}$. Experiments were run under 1 atm nitrogen with a ramp rate of $10^{\circ} \mathrm{C} / \mathrm{min}$. Sample sizes were between $5-7 \mathrm{mg}$. 
An isothermal TGA of 2 at $110^{\circ} \mathrm{C}$ showed the first mass loss feature to plateau at about $60 \%$ residual mass (Figure 7.10 in Supporting Information). The NHC and HMDS moieties account for $45 \%$ and $40 \%$ of the total mass, respectively. ${ }^{1} \mathrm{H}$ and ${ }^{13} \mathrm{C} \mathrm{NMR}$ analysis in $\mathrm{CDCl}_{3}$ of the residual material found peaks with chemical shifts matching exactly those for $\left[\mathrm{I}^{t} \mathrm{Bu}_{2}\right] \mathrm{CuCl} .{ }^{35}$ No remnant peaks for HMDS were observed. The protons residing in the unsaturated backbone of imidazolylidenes are known to be acidic. They are capable of undergoing deuterium exchange, ${ }^{36}$ silylation, ${ }^{37}$ and metalation affording an anionic N-heterocyclic dicarbene (NHDC). ${ }^{38}$ Our proposed decomposition route for $\mathbf{2}$ is via deprotonation of the unsaturated backbone and elimination of hexamethyldisilazane forming an anionic NHDC species (Figure 7.7). The $\left[\mathrm{I}^{t} \mathrm{Bu}_{2}\right] \mathrm{CuCl}$ species which was identified during NMR analysis likely formed due to $\mathrm{HCl}$ in the $\mathrm{CDCl}_{3}$ and verifies that the $\mathrm{N}$-alkyl substituents of $\mathrm{I}^{t} \mathrm{Bu}_{2}$ were intact.
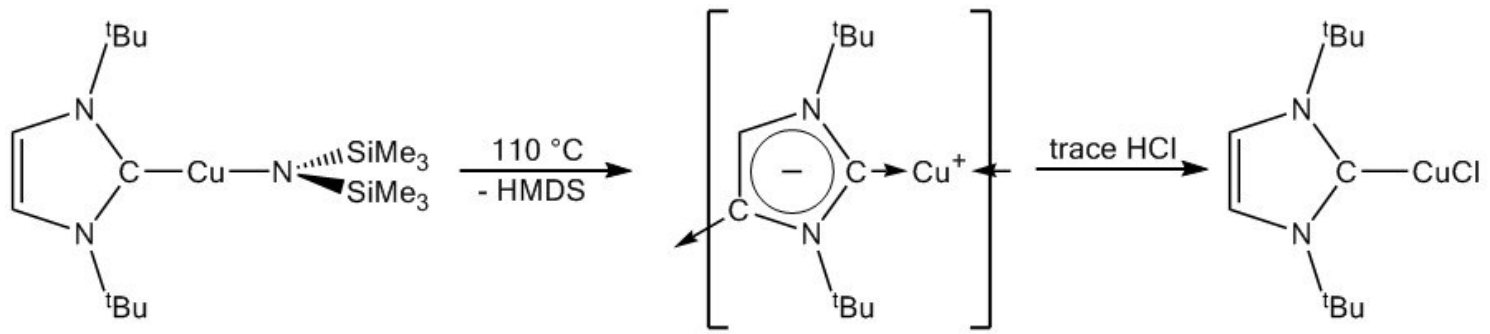

Figure 7.7 Thermal Decomposition of $\left[\mathrm{I}^{t} \mathrm{Bu}_{2}\right] \mathrm{Cu}(\mathrm{I}) \mathrm{HMDS}(2)$ via Deportonation of the Unsaturated NHC Backbone and Elimination of Hexamethyldisilazane.

An NHC with a methylated unsaturated backbone such as in $\mathrm{I}^{i} \mathrm{Pr}_{2} \mathrm{Me}_{2}$ would prevent this type of decomposition. Compound $\mathbf{3}$ sublimed in excellent yield and displayed a single weight loss feature (indicative of volatilization) and a low residual mass of $4.3 \%$ in a TG experiment (Figure 7.6). The trend in thermal stability of the series $\mathbf{1}$ - $\mathbf{3}$ supports our purposed decomposition pathway for $\mathbf{2}$ and demonstrates how this 
thermal reactivity can be kinetically avoided by increasing the steric bulk of the $\mathrm{N}$-alkyl groups or prevented by methylation of the backbone.

In light of the vulnerability of the unsaturated NHCs, several imidazolinylidenes were studied in $4-7$. NHCs with saturated backbones resulted in volatile compounds with excellent thermal stability. A comparison of the residual masses from TG experiments of $\mathbf{3}(4.3 \%)$ to $\mathbf{5}(1.0 \%)$ (Figure 7.6$)$ highlights the superior thermal stability of the imidazolinylidenes versus imidazolylidenes. Thermal rearrangements for $I^{i} \mathrm{Pr}_{2} \mathrm{Me}_{2}$ and $\mathrm{I}^{t} \mathrm{Bu}_{2}$ occurring through a $\mathrm{C}-\mathrm{N}$ bond activation have been shown for transition metal complexes. ${ }^{39}$ To our knowledge, similar chemistry has not been demonstrated for imidazolinylidenes which possibly explains their increased stability. The alkyl substituents for the series of $\mathbf{4}-\mathbf{7}$ were chosen in order to study the effect of steric bulk on thermal stability. The residual masses for these compounds were similar and found between $0.7-1.5 \%$ (Figure 7.11 in Supporting Information).

The contrasting thermal stabilities between the unsaturated and saturated NHC compounds prompted the selection of ADCs to explore whether the backbone could be removed while maintaining desirable characteristics of an ALD precursor. TG experiments of $\mathbf{8}-\mathbf{1 0}$ yielded low residual masses between $0.4-1.6 \%$ (Figure 7.12 in Supporting Information) which demonstrates thermal stability and volatility. The cyclic nature was thus determined to be an elective feature for the diamino carbene in the design of these precursors.

Further thermal characterisation of the volatile compounds $(\mathbf{3}-\mathbf{1 0})$ was accomplished by a thermal stress test using the TGA. This test simply varied the sample mass over several experiments with a constant heating rate. Consequently, the larger 
samples are exposed to higher temperatures and more extensive decomposition can occur. Evidently, evaporation rates play a role and the more volatile compounds have a slight advantage because lower temperatures are achieved within the window of evaporation. The thermal stress test is relatively quick to perform compared to other solution based quantitative methods and generates similar qualitative trends. ${ }^{40}$ Different molar amounts were heated at $10{ }^{\circ} \mathrm{C} / \mathrm{min}$ and the residual mass was converted to a percentage of decomposition assuming that the residual material was entirely copper. Compounds 3 10 showed values for percent decomposition between $2-8 \%$ at low sample masses and between $11-86 \%$ at high sample masses (Figure 7.8).

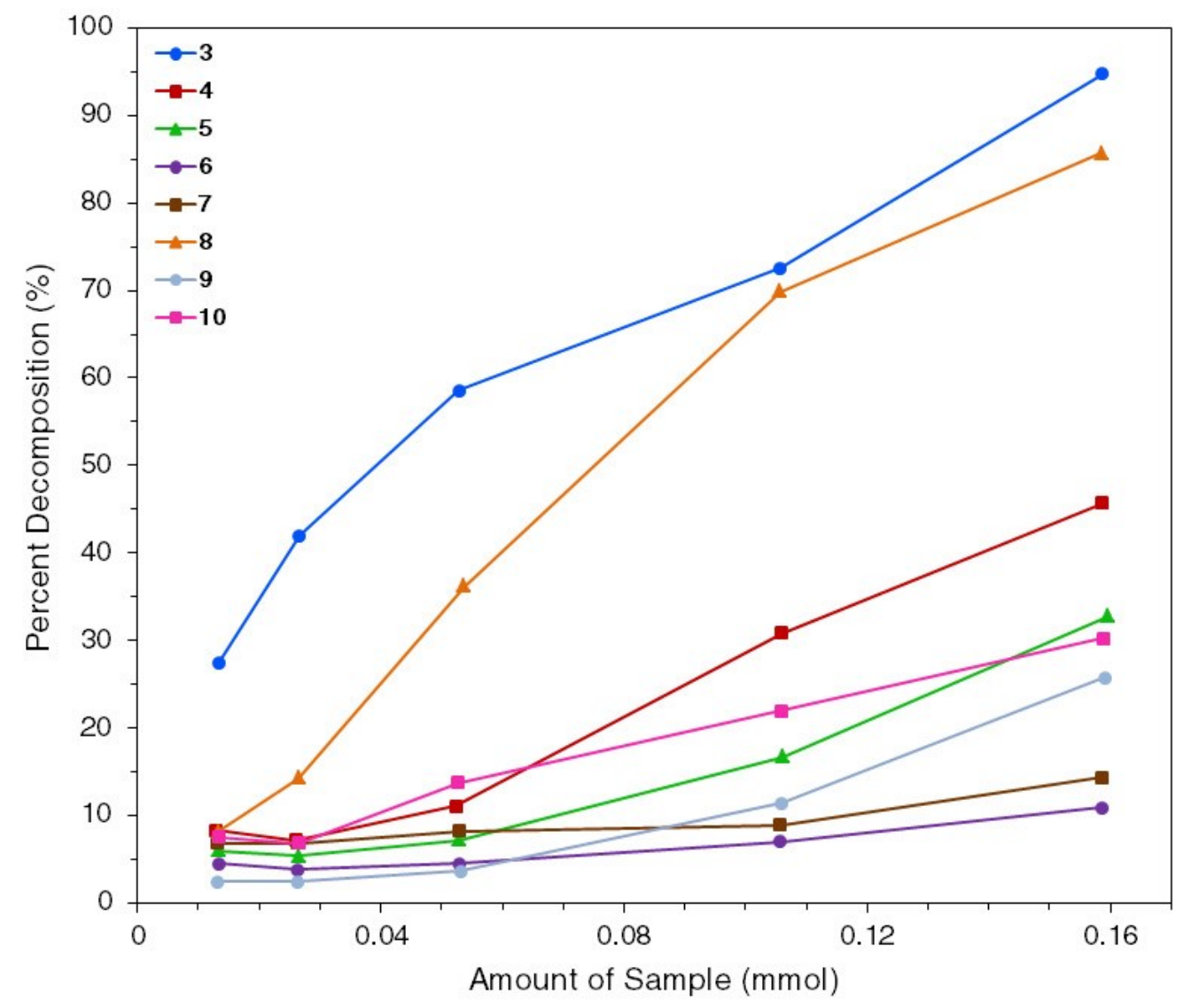

Figure 7.8 TGA thermal stress test for $3-\mathbf{1 0}$. The heating rate was $10{ }^{\circ} \mathrm{C} / \mathrm{min}$ for each experiment. The smallest sample sizes were $1.3 \mu \mathrm{mol}(\sim 5 \mathrm{mg})$ and the largest were 15.9 $\mu \mathrm{mol}(\sim 60 \mathrm{mg})$. 
For compounds $4-\mathbf{7}$, imidazolinylidenes with bulkier $\mathrm{N}$-substituents imparted more thermal stability to the respective copper complex. A similar trend was observed for the formamidinylidenes $\mathbf{8}$ and $\mathbf{9}$; however, $\mathbf{1 0}$ showed more decomposition than $\mathbf{9}$. This dependence of thermal stability on steric bulk suggests that dissociation of the carbenecopper bond is not the first step during decomposition. Hillier et al. reported that bond dissociation enthalphies are less for sterically demanding NHCs in the $\mathrm{Cp} * \mathrm{Ru}(\mathrm{NHC}) \mathrm{Cl}$ system. ${ }^{41}$ Steric bulk within these copper complexes may be preventing oligomerization, intermolecular disproportionation or possibly a $\beta$-methyl ${ }^{42}$ or $\gamma-\mathrm{H}$ abstraction ${ }^{43}$ of the hexamethyldisilazide ligand.

The thermal stress test for $\mathbf{1 0}$ was strikingly different compared to the others (Figure 7.13 in Supporting Information). Two distinct weight loss features were observed and the inflection point appeared at a higher weight percent for higher sample mass loading. The steric bulk of $\mathrm{A}^{i} \mathrm{Pr}_{4}$ may be a tipping point for the dissociation of the carbene copper bond. During the temperature of the experiment the rate of $\mathrm{Cu}-\mathrm{A}^{i} \mathrm{Pr}_{4}$ bond dissociation would accelerate. Therefore, when large amounts of $\mathbf{1 0}$ are exposed to high temperatures larger amounts of uncoordinated copper amide would remain in the sample pan. Furthermore, free ${ }^{44}$ and coordinated ${ }^{28 d} \mathrm{~A}^{i} \operatorname{Pr}_{4}$ have been found to rearrange to formamidine via propene elimination which would result in a similar outcome as bond dissociation.

Compound $\mathbf{5}$ was reported as an ALD precursor with excellent thermal stability over two weeks when heated at $130{ }^{\circ} \mathrm{C}$. The thermal stress test via TGA showed compounds 6, 7 and 9 to behave as well or better than 5 and predicts these compounds to be ideal precursors for ALD. 
Stepped isothermal TGA experiments were performed to determine and compare rates of evaporation. These experiments were performed for compounds $\mathbf{3}-\mathbf{1 0}$ which were thermally stable within a suitable temperature range $\left(110-170^{\circ} \mathrm{C}\right)$ and time frame of the experiment. The results show a general trend where compounds of lower molecular mass show higher rates of evaporation (Figure 7.9). Interestingly, 7 showed higher rates of evaporation than $\mathbf{6}$ even though 7 is higher in mass by two methyl groups. A similar reversal is evident for $\mathbf{4}$ and $\mathbf{5}$, which also differ by two methyl groups. A likely explanation is that intermolecular van der Waal interactions are disrupted by the addition of methyl groups. This explanation is counterintuitive since increasing the number of alkyl groups would likely increase the number of van der Waal interactions. ${ }^{45}$ However, the placement of the methyl groups in $\mathbf{5}$ and $\mathbf{7}$ are onto methylene carbons adjacent to unobstructed methylene or methyl carbons. Branching at these particular carbons prevents a chain of intermolecular interactions and would lower the overall number of interactions available between two molecules. The higher rates of evaporation for 5 and $\mathbf{7}$ highlight how judicious alkyl groups can be added to increase evaporation rates. 


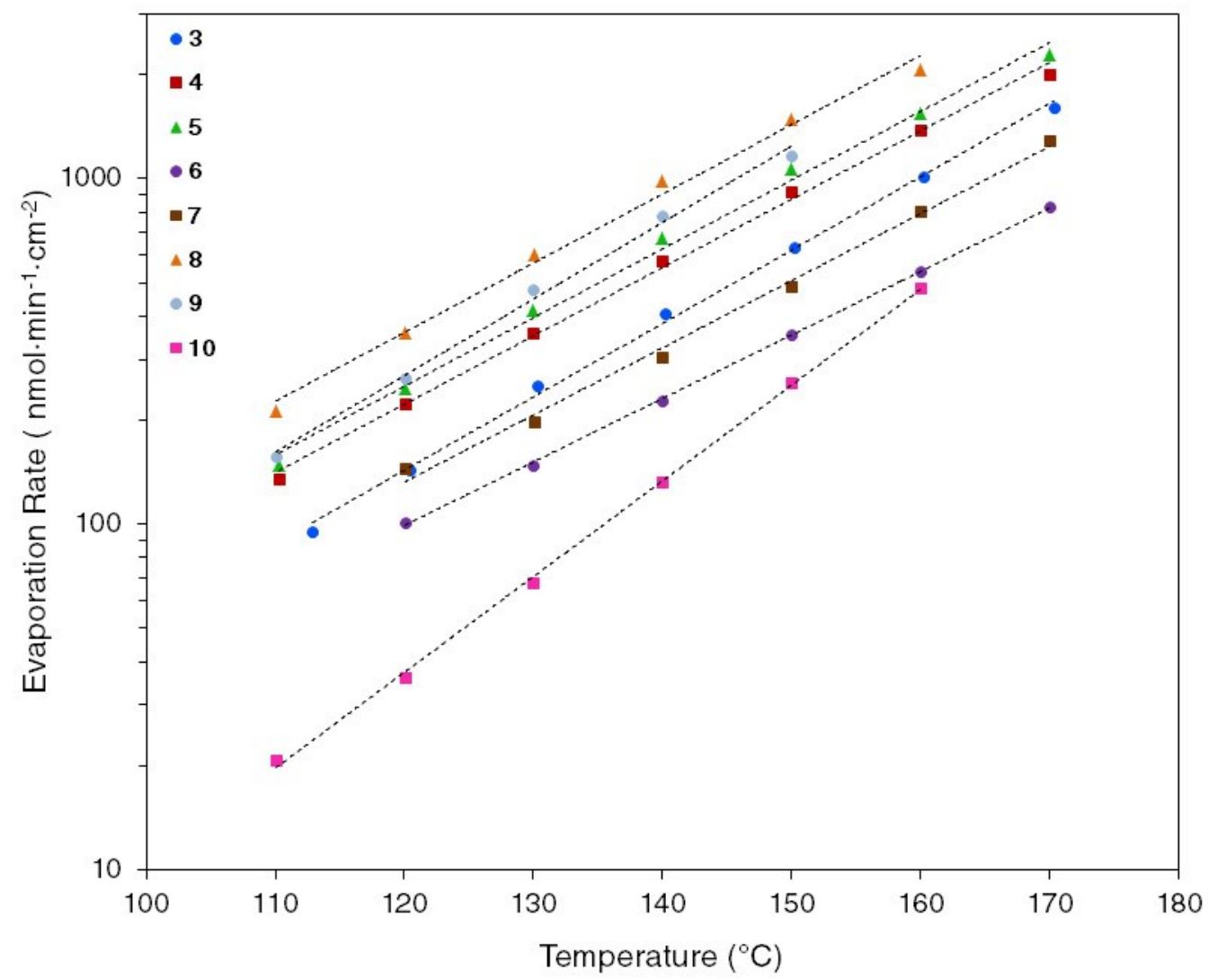

Figure 7.9 Isothermal rates of vaporization for $\mathbf{3}-\mathbf{1 0}$ under $1 \mathrm{~atm}$ of nitrogen.

Vapour pressure data was calculated from the data shown in Figure 7.9 by using the Langmuir equation. ${ }^{46}$ The Langmuir equation provides an equality between the rate of mass loss of a substance and its vapour pressure in a vacuum. To account for the flowing gas environment of the TGA instrument, a calibration constant was determined using benzoic acid as a standard. The 1 Torr temperatures for $\mathbf{3 - 1 0}$ were calculated to be between $143-172{ }^{\circ} \mathrm{C}$ (Table 7.1) ${ }^{47}$ By this method, the 1 Torr value for copper(II)TMHD was $167{ }^{\circ} \mathrm{C}$ which is within $3 \%$ of a measured value of $172{ }^{\circ} \mathrm{C} .{ }^{48}$

The vapour pressure curves showed Arrhenius temperature dependence $\left(\mathrm{R}^{2}>\right.$ $0.99)$ for all compounds tested. Enthalpies of vapourization $\left(\Delta \mathrm{H}_{\text {vap }}\right)$ could be extracted using the Classius-Clapeyron equation. The $\Delta \mathrm{H}_{\text {vap }}$ values for 3-9 (Table 7.1) varied 
slightly and were found between $63-72 \mathrm{~kJ} / \mathrm{mol}$. Compound 10 yielded a $\Delta \mathrm{H}_{\text {vap value of }}$ $89.6 \mathrm{~kJ} / \mathrm{mol}$ but an accurate determination was complicated by its concomitant decomposition. The $\Delta \mathrm{H}_{\mathrm{vap}}$ values for 3-9 compare well with dimeric copper(I) amidinates $(58.4-82.4 \mathrm{~kJ} / \mathrm{mol})^{4 \mathrm{a}}$ and are lower than monomeric copper(II) $\beta$-diketonates $(81-151 \mathrm{~kJ} / \mathrm{mol}){ }^{49}$ The accuracy of $\Delta \mathrm{H}_{\mathrm{vap}}$ measurements were confirmed with copper(II)TMHD whose sublimation enthalpy was measured to be $103.9 \mathrm{~kJ} / \mathrm{mol}$ which is in good agreement to literature values measured by similar methods. ${ }^{48-50}$

\subsection{Conclusion}

A series of NHC and ADC copper(I) hexamethyldisilazide complexes were synthesized and characterized with a focus on their thermal properties. The carbene and amide ligands were installed onto the metal center via a single step reaction. The complexes were monomeric in the gas and solid phase validating the role of the carbene. All compounds were volatile during vacuum sublimation or distillation. Ramped TGA for 3 - 10 displayed a single weight loss feature resulting in low residual masses demonstrating these compounds to be thermally stable during volatilization.

The thermal stability of the complexes was strongly dependent on the character of the carbon-carbon carbene backbone. Steric bulk of the N-substituents was also a significant factor determining the thermal stability of the complexes. The strength of the carbene-copper bond was verified as it remained intact at elevated temperature $(>175$ ${ }^{\circ} \mathrm{C}$ ) during TGA. Dissociation of the carbene-copper bond was not suspected to be the operative step during decomposition at higher temperatures.

There was a striking difference between the thermal stabilities of the imidazolylidenes complexes compared to those of the imidazolinylidenes. The thermal 
stability of a formamidinylidene complex could be equal to either of the aforementioned NHC complexes depending on the steric bulk of the N-alkyl substituents. The results presented here will be useful guidelines for the design of other metal precursors employing diamino carbenes or to areas of organometallic catalysis where thermal stability is of interest.

\subsection{Experimental Section}

General Considerations. All manipulations involving the synthesis and handling of copper(I) compounds were performed in an MBraun Labmaster 130 Drybox under a nitrogen atmosphere. All reagents were purchased from either Aldrich Chemical Co. or Alfa Aesar and used as received. Anhydrous tetrahydrofuran and pentane were purchased from Aldrich Chemical Co. and used as received. The hydrochloride salts of the NHCs and ADCs were prepared according to literature: $\mathrm{I}^{i} \mathrm{Pr}_{2},{ }^{51} \mathrm{I}^{t} \mathrm{Bu}_{2},{ }^{36} \mathrm{SI}^{i} \mathrm{Pr}_{2},{ }^{52} \mathrm{SI}^{t} \mathrm{Bu}_{2},{ }^{53}$ $\mathrm{AMe}_{4},{ }^{54} \mathrm{~A}^{i} \mathrm{Pr}_{4} .{ }^{20 \mathrm{a}}$ Preparation of the hydrochloride salts for $\mathrm{AEt}_{4}, \mathrm{SI}^{t} \mathrm{Bu}_{2} \mathrm{Me}_{2}$, and $\mathrm{SIEt}_{2}$ are provided below. Compound $\mathbf{3}^{8}$ and $\mathrm{Et}_{2} \mathrm{O} \cdot \mathrm{LiN}\left(\mathrm{SiMe}_{3}\right)_{2}{ }^{55}$ were prepared according to literature.

Physical Measurements. NMR spectra were recorded on a $400 \mathrm{MHz}$ Bruker AMX spectrometer. NMR spectra were measured in $\mathrm{C}_{6} \mathrm{D}_{6}$ and were referenced against residual protonated solvent. Thermogravimeteric analysis was performed on a TA Instrument Q50 apparatus located in an MBraun Labmaster 130 Dry box under a nitrogen atmosphere in platinum crucibles. Evaporation rates were recorded from a steppedisotherm TG experiment where isotherms between $110-170{ }^{\circ} \mathrm{C}$ were maintained for 10 min and sample masses were $20-40 \mathrm{mg}$. Melting points were measured in capillaries sealed with vacuum grease using a Mel-Temp II apparatus. High resolution mass 
spectrometry was performed on a Kratos Concept - Accurate Mass EI at the John L. Homes Mass Spectrometry Facility at the University of Ottawa.

\section{1,3-diisopropyl-imidazol-2-ylidene copper hexamethyldisilazide (1): 1,3-}

diisopropylimidazolium chloride was suspened in $30 \mathrm{~mL}$ of $\mathrm{THF}$ and $\mathrm{CuCl}$ was added. The suspension was stirred for $1 \mathrm{~h}$ during which it cleared to a solution.

$\mathrm{Et}_{2} \mathrm{O} \cdot \mathrm{LiN}\left(\mathrm{SiMe}_{3}\right)_{2}$ dissolved in $10 \mathrm{ml}$ of $\mathrm{THF}$ was added dropwise and stirring proceeded for $18 \mathrm{~h}$. Volatiles were stripped off under reduced pressure. The residual material was taken up in $10 \mathrm{~mL}$ of pentane. The insoluble solids were filtered off and washed with $2 \mathrm{x}$ $2 \mathrm{~mL}$ of pentane. All pentane fractions were combined and the pentane was stripped off under vacuum to afford a light yellow liquid (0.582 g, $77.6 \%$ ). ${ }^{1} \mathrm{H}$ NMR (400 MHz, $\left.\mathrm{C}_{6} \mathrm{D}_{6}\right): \delta 0.62\left[\mathrm{~s}, 18 \mathrm{H}, \mathrm{N}\left(\mathrm{Si}\left(\mathrm{CH}_{3}\right)_{3}\right)_{2}\right], \delta 0.95\left[\mathrm{~d}, 12 \mathrm{H}, \mathrm{NCH}\left(\mathrm{CH}_{3}\right)_{2}\right], \delta 4.69$ [sept, $2 \mathrm{H}$, $\left.\mathrm{NCH}\left(\mathrm{CH}_{3}\right)_{2}\right], \delta 6.05\left[\mathrm{~s}, 2 \mathrm{H}, \mathrm{N}(\mathrm{CH})_{2} \mathrm{~N}\right] .{ }^{13} \mathrm{C} \mathrm{NMR}\left(100 \mathrm{MHz}, \mathrm{C}_{6} \mathrm{D}_{6}\right): \delta 7.3$

$\left[\mathrm{N}\left(\mathrm{Si}\left(\mathrm{CH}_{3}\right)_{3}\right)_{2}\right], \delta 23.4\left[\mathrm{NCH}\left(\mathrm{CH}_{3}\right)_{2}\right], \delta 53.2\left[\mathrm{NCH}\left(\mathrm{CH}_{3}\right)_{2}\right], \delta 116.1[\mathrm{~N}(\mathrm{CH}) 2 \mathrm{~N}], \delta 176.9$ [NCN]. Compound purity was unsatisfactory for elemental analysis.

\section{1,3-ditertbutyl-imidazol-2-ylidene copper hexamethyldisilazide (2): Compound $\mathbf{2}$}

was prepared in an analogous manner as compound $\mathbf{1}$ substituting: 1,3-ditertbutylimidazolium chloride (0.396 g, $1.83 \mathrm{mmol}), \mathrm{CuCl}(0.186 \mathrm{~g}, 1.88 \mathrm{mmol})$, $\mathrm{Et}_{2} \mathrm{O} \cdot \mathrm{LiN}\left(\mathrm{SiMe}_{3}\right)_{2}(0.882 \mathrm{~g}, 3.65 \mathrm{mmol})$. Compound 2 was purified by sublimation (95 $\left.{ }^{\circ} \mathrm{C}, 20 \mathrm{mtorr}\right)$ and collected as a colourless solid (0.612 g, 82.9\%). ${ }^{1} \mathrm{H}$ NMR (400 MHz, $\left.\mathrm{C}_{6} \mathrm{D}_{6}\right): \delta 0.55\left[\mathrm{~s}, 18 \mathrm{H}, \mathrm{N}\left(\mathrm{Si}\left(\mathrm{CH}_{3}\right)_{3}\right)_{2}\right], \delta 1.46\left[\mathrm{~s}, 18 \mathrm{H}, \mathrm{NC}\left(\mathrm{CH}_{3}\right)_{3}\right], \delta 6.33[\mathrm{~s}, 2 \mathrm{H}$, $\left.\mathrm{N}\left(\mathrm{CH}_{2}\right) \mathrm{N}\right] .{ }^{13} \mathrm{C} \mathrm{NMR}\left(100 \mathrm{MHz}, \mathrm{C}_{6} \mathrm{D}_{6}\right): \delta 6.9\left[\mathrm{~N}\left(\mathrm{Si}\left(\mathrm{CH}_{3}\right)_{3}\right)_{2}\right), \delta 31.8\left(\mathrm{NC}\left(\mathrm{CH}_{3}\right)_{3}\right], \delta 57.6$ $\left[\mathrm{NC}\left(\mathrm{CH}_{3}\right)_{3}\right], \delta 115.7\left[\mathrm{~N}\left(\mathrm{CH}_{2}\right) \mathrm{N}\right], \delta 176.8[\mathrm{NCN}] \mathrm{HRMS}(\mathrm{EI}) \mathrm{m} / \mathrm{z}$ calcd for $\mathrm{C}_{17} \mathrm{H}_{38} \mathrm{~N}_{3} \mathrm{Si}_{2} \mathrm{Cu} \mathrm{M}^{+} 403.1900$, found 403.1883 . 


\section{1,3-diethyl-imidazolin-2-ylidene copper hexamethyldisilazide (4): Compound 4}

was prepared in an analogous manner as compound $\mathbf{1}$ substituting: 1,3-diethylimidazolinium chloride (1.067 g, $6.55 \mathrm{mmol}), \mathrm{CuCl}$ (0.668 g, $6.75 \mathrm{mmol})$, $\mathrm{Et}_{2} \mathrm{O} \cdot \mathrm{LiN}\left(\mathrm{SiMe}_{3}\right)_{2}(3.161 \mathrm{~g}, 13.09 \mathrm{mmol})$. Compound $\mathbf{4}$ was purified by distillation (158 ${ }^{\circ} \mathrm{C}, 20$ mtorr) and collected as a colourless liquid (1.780 g, $77.6 \%$ ). ${ }^{1} \mathrm{H}$ NMR (400 MHz, $\left.\mathrm{C}_{6} \mathrm{D}_{6}\right): \delta 0.57\left[\mathrm{~s}, 18 \mathrm{H}, \mathrm{N}\left(\mathrm{Si}\left(\mathrm{CH}_{3}\right)_{3}\right)_{2}\right], \delta 0.76\left[\mathrm{t}, 6 \mathrm{H}, \mathrm{NCH}_{2} \mathrm{CH}_{3}\right], \delta 2.40[\mathrm{~s}, 4 \mathrm{H}$, $\left.\mathrm{N}\left(\mathrm{CH}_{2}\right)_{2} \mathrm{~N}\right], \delta 3.22\left[\mathrm{q}, 4 \mathrm{H}, \mathrm{NCH}_{2} \mathrm{CH}_{3}\right] .{ }^{13} \mathrm{C} \mathrm{NMR}\left(100 \mathrm{MHz}, \mathrm{C}_{6} \mathrm{D}_{6}\right): \delta 7.6\left[\mathrm{~N}\left(\mathrm{Si}\left(\mathrm{CH}_{3}\right)_{3}\right)_{2}\right]$, $\delta 14.2\left[\mathrm{NCH}_{2} \mathrm{CH}_{3}\right], \delta 45.2\left[\mathrm{~N}\left(\mathrm{CH}_{2}\right)_{2} \mathrm{~N}\right], \delta 47.5\left[\mathrm{NCH}_{2} \mathrm{CH}_{3}\right], \delta 201.6[\mathrm{NCN}] \mathrm{HRMS}(\mathrm{EI})$ $\mathrm{m} / \mathrm{z}$ calcd for $\mathrm{C}_{13} \mathrm{H}_{32} \mathrm{~N}_{3} \mathrm{Si}_{2} \mathrm{Cu} \mathrm{M} \mathrm{M}^{+}$349.1431, found 349.1464.

\section{1,3-diisopropyl-imidazolin-2-ylidene copper hexamethyldisilazide (5): Compound}

5 was prepared in an analogus manner as compound $\mathbf{1}$ substituting: 1,3 diisopropylimidazolinium chloride (0.347 g, $1.82 \mathrm{mmol}), \mathrm{CuCl}$ (0.186 g, $1.88 \mathrm{mmol})$, $\mathrm{Et}_{2} \mathrm{O} \cdot \mathrm{LiN}\left(\mathrm{SiMe}_{3}\right)_{2}(0.879 \mathrm{~g}, 3.64 \mathrm{mmol})$. Compound $\mathbf{5}$ was purified by sublimation employing a dry ice cold finger $\left(93^{\circ} \mathrm{C}, 35 \mathrm{mtorr}\right)$ and collected as a colourless solid (0.592 g, 86.0 \%); m.p. $49-51{ }^{\circ} \mathrm{C}$. Analytical data matched previously reported data. ${ }^{8}{ }^{1} \mathrm{H}$ NMR (400 MHz, $\left.\mathrm{C}_{6} \mathrm{D}_{6}\right): \delta 4.46$ [sept, 2H, $\left.\mathrm{NCH}\left(\mathrm{CH}_{3}\right)_{2}\right], \delta 2.50\left[\mathrm{~s}, 4 \mathrm{H}, \mathrm{N}\left(\mathrm{CH}_{2}\right)_{2} \mathrm{~N}\right], \delta 0.81$ $\left[\mathrm{d}, 12 \mathrm{H}, \mathrm{NCH}\left(\mathrm{CH}_{3}\right)_{2}\right], \delta 0.54\left[\mathrm{~s}, 18 \mathrm{H}, \mathrm{N}\left(\mathrm{Si}\left(\mathrm{CH}_{3}\right)_{3}\right)_{2}\right] .{ }^{13} \mathrm{C} \mathrm{NMR}\left(100 \mathrm{MHz}, \mathrm{C}_{6} \mathrm{D}_{6}\right): \delta$ $200.4[\mathrm{NCN}], \delta 51.2\left[\mathrm{NCH}\left(\mathrm{CH}_{3}\right)_{2}\right], \delta 41.9\left[\mathrm{~N}\left(\mathrm{CH}_{2}\right)_{2} \mathrm{~N}\right], \delta 20.7[\mathrm{NCH}(\mathrm{CH} 3) 2], \delta 7.2$ [N( $\left.\left.\mathrm{Si}\left(\mathrm{CH}_{3}\right)_{3}\right)_{2}\right]$. HRMS (EI) m/z calcd for $\mathrm{C}_{15} \mathrm{H}_{36} \mathrm{~N}_{3} \mathrm{Si}_{2} \mathrm{Cu} \mathrm{M}^{+}$377.1744, found 377.1763.

1, 3-ditertbutyl-imidazolin-2-ylidene copper hexamethyldisilazide (6): Compound 6 was prepared in an analogus manner as compound 1 substituting: 1,3-ditertbutylimidazolinium chloride ( $0.350 \mathrm{~g}, 1.60 \mathrm{mmol}), \mathrm{CuCl}(0.163 \mathrm{~g}, 1.65 \mathrm{mmol})$, $\mathrm{Et}_{2} \mathrm{O} \cdot \mathrm{LiN}\left(\mathrm{SiMe}_{3}\right)_{2}(0.773 \mathrm{~g}, 3.20 \mathrm{mmol})$. Compound $\mathbf{6}$ was purified by sublimation (91 
${ }^{\circ} \mathrm{C}, 20$ mtorr) and collected as a colourless solid (0.548 g, $\left.84.3 \%\right)$; m.p. $96-98{ }^{\circ} \mathrm{C} .{ }^{1} \mathrm{H}$ NMR $\left(400 \mathrm{MHz}, \mathrm{C}_{6} \mathrm{D}_{6}\right): \delta 0.56\left[\mathrm{~s}, 18 \mathrm{H}, \mathrm{N}\left(\mathrm{Si}\left(\mathrm{CH}_{3}\right)_{3}\right)_{2}\right], \delta 1.33\left[\mathrm{~s}, 18 \mathrm{H}, \mathrm{NC}\left(\mathrm{CH}_{3}\right)_{3}\right], \delta 2.59$ $\left[\mathrm{s}, 4 \mathrm{H}, \mathrm{N}\left(\mathrm{CH}_{2}\right)_{2} \mathrm{~N}\right] .{ }^{13} \mathrm{C} \mathrm{NMR}\left(100 \mathrm{MHz}, \mathrm{C}_{6} \mathrm{D}_{6}\right): \delta 6.9\left[\mathrm{~N}\left(\mathrm{Si}\left(\mathrm{CH}_{3}\right)_{3}\right)_{2}\right], \delta 30.8\left[\mathrm{NC}\left(\mathrm{CH}_{3}\right)_{3}\right]$, $\delta 45.2\left[\mathrm{~N}\left(\mathrm{CH}_{2}\right)_{2} \mathrm{~N}\right], \delta 55.0\left[\mathrm{NC}\left(\mathrm{CH}_{3}\right)_{3}\right], \delta 201.5[\mathrm{NCN}] \mathrm{HRMS}(\mathrm{EI}) \mathrm{m} / \mathrm{z}$ calcd for $\mathrm{C}_{17} \mathrm{H}_{40} \mathrm{~N}_{3} \mathrm{Si}_{2} \mathrm{Cu} \mathrm{M}{ }^{+}$405.2057, found 405.2031.

\section{4, 5-dimethyl-1, 3-ditertbutyl-imidazolin-2-ylidene copper hexamethyldisilazide}

(7): Compound 7 was prepared in an analogus manner as compound $\mathbf{1}$ substituting: 4,5dimethyl-1,3-ditertbutyl-imidazolinium chloride (0.425 g, $1.72 \mathrm{mmol}), \mathrm{CuCl}$ (0.176 g, $1.78 \mathrm{mmol}), \mathrm{Et}_{2} \mathrm{O} \cdot \mathrm{LiN}\left(\mathrm{SiMe}_{3}\right)_{2}(0.832 \mathrm{~g}, 3.45 \mathrm{mmol})$. Compound 7 was purified by sublimation $\left(75^{\circ} \mathrm{C}, 10\right.$ mtorr $)$ and collected as a colourless solid ( $\left.0.555 \mathrm{~g}, 74.0 \%\right)$; m.p. $118{ }^{\circ} \mathrm{C} .{ }^{1} \mathrm{H}$ NMR $\left(400 \mathrm{MHz}, \mathrm{C}_{6} \mathrm{D}_{6}\right): \delta 0.55\left[\mathrm{~d}, 6 \mathrm{H}, \mathrm{N}\left(\mathrm{CHCH}_{3}\right)_{2} \mathrm{~N}\right], \delta 0.57[\mathrm{~s}, 18 \mathrm{H}$, $\left.\mathrm{N}\left(\mathrm{Si}\left(\mathrm{CH}_{3}\right)_{3}\right)_{2}\right], \delta 1.43\left[\mathrm{~s}, 18 \mathrm{H}, \mathrm{NC}\left(\mathrm{CH}_{3}\right)_{3}\right], \delta 2.77\left[\mathrm{q}, 2 \mathrm{H}, \mathrm{N}\left(\mathrm{CHCH}_{3}\right)_{2} \mathrm{~N}\right] .{ }^{13} \mathrm{C} \mathrm{NMR}(100$ $\left.\mathrm{MHz}, \mathrm{C}_{6} \mathrm{D}_{6}\right): \delta 7.2\left[\mathrm{~N}\left(\mathrm{Si}\left(\mathrm{CH}_{3}\right)_{3}\right)_{2}\right], \delta 21.0\left[\mathrm{~N}\left(\mathrm{CHCH}_{3}\right)_{2} \mathrm{~N}\right], \delta 32.7\left[\mathrm{NC}\left(\mathrm{CH}_{3}\right)_{3}\right], \delta 55.2$ $\left[\mathrm{NC}\left(\mathrm{CH}_{3}\right)_{3}\right], \delta 62.3\left[\mathrm{~N}\left(\mathrm{CHCH}_{3}\right)_{2} \mathrm{~N}\right], \delta 199.0[\mathrm{NCN}] \mathrm{HRMS}(\mathrm{EI}) \mathrm{m} / \mathrm{z}$ calcd for $\mathrm{C}_{19} \mathrm{H}_{44} \mathrm{~N}_{3} \mathrm{Si}_{2} \mathrm{Cu} \mathrm{M}{ }^{+}$433.2370, found 433.2359.

$N, N, N^{\prime}, N^{\prime}$-tetramethylformamidinylidene copper hexamethyldisilazide (8):

Compound $\mathbf{8}$ was prepared in an analogous manner as compound $\mathbf{1}$ substituting: N,N,N',N'-tetramethylformamidinium chloride $(4.21 \mathrm{~g}, 30.8 \mathrm{mmol})$ suspended in $100 \mathrm{ml}$ of THF, $\mathrm{CuCl}(3.15 \mathrm{~g}, 31.8 \mathrm{mmol}), \mathrm{Et}_{2} \mathrm{O} \cdot \mathrm{LiN}\left(\mathrm{SiMe}_{3}\right)_{2}(14.90 \mathrm{~g}, 61.7 \mathrm{mmol})$ dissolved in $60 \mathrm{~mL}$ THF. $30 \mathrm{~mL}$ of pentane was used for the extraction and $3 \times 10 \mathrm{~mL}$ portions of pentane were used for washing. Compound $\mathbf{8}$ was purified by sublimation $\left(90^{\circ} \mathrm{C}, 20\right.$ mtorr) and collected as a colourless solid (8.746 g, 87.5 \%). ${ }^{1} \mathrm{H}$ NMR (400 MHz, $\left.\mathrm{C}_{6} \mathrm{D}_{6}\right): \delta$ $0.56\left[\mathrm{~s}, 18 \mathrm{H}, \mathrm{N}\left(\mathrm{Si}\left(\mathrm{CH}_{3}\right)_{3}\right)_{2}\right], \delta 2.43\left[\mathrm{~s}, 12 \mathrm{H}, \mathrm{N}\left(\mathrm{CH}_{3}\right)_{2}\right] .{ }^{13} \mathrm{C} \mathrm{NMR}\left(100 \mathrm{MHz}, \mathrm{C}_{6} \mathrm{D}_{6}\right): \delta 7.2$ 
$\left[\mathrm{N}\left(\mathrm{Si}\left(\mathrm{CH}_{3}\right)_{3}\right)_{2}\right], \delta 44.0\left[\mathrm{~N}\left(\mathrm{CH}_{3}\right)_{2}\right], \delta 214.1[\mathrm{NCN}] \mathrm{HRMS}(\mathrm{EI}) \mathrm{m} / \mathrm{z}$ calcd for $\mathrm{C}_{11} \mathrm{H}_{30} \mathrm{~N}_{3} \mathrm{Si}_{2} \mathrm{Cu} \mathrm{M} \mathrm{M}^{+}$323.1274, found 323.1271.

\section{$N, N, N^{\prime}, N^{\prime}$-tetraethylformamidinylidene copper hexamethyldisilazide (9):}

Compound $\mathbf{9}$ was prepared in an analogous manner as compound $\mathbf{1}$ substituting: N,N,N',N'-tetraethylformamidinium chloride (0.760 g, 3.94 mmol), $\mathrm{CuCl}(0.403 \mathrm{~g}, 4.07$ $\mathrm{mmol}), \mathrm{Et}_{2} \mathrm{O} \cdot \mathrm{LiN}\left(\mathrm{SiMe}_{3}\right)_{2}(1.905 \mathrm{~g}, 7.89 \mathrm{mmol})$. Compound 9 was purified by distillation $\left(148{ }^{\circ} \mathrm{C}, 10 \mathrm{mtorr}\right)$ and collected as a light yellow liquid (1.010 g, $\left.67.3 \%\right) .{ }^{1} \mathrm{H}$ NMR (400 $\left.\mathrm{MHz}, \mathrm{C}_{6} \mathrm{D}_{6}\right): \delta 0.56\left[\mathrm{~s}, 18 \mathrm{H}, \mathrm{N}\left(\mathrm{Si}\left(\mathrm{CH}_{3}\right)_{3}\right)_{2}\right], \delta 0.80\left[\mathrm{t}, 12 \mathrm{H}, \mathrm{N}\left(\mathrm{CH}_{2} \mathrm{CH}_{3}\right)_{2}\right], \delta 3.07$ [q, $8 \mathrm{H}$, $\left.\mathrm{N}\left(\mathrm{CH}_{2} \mathrm{CH}_{3}\right)_{2}\right] .{ }^{13} \mathrm{C} \mathrm{NMR}\left(100 \mathrm{MHz}, \mathrm{C}_{6} \mathrm{D}_{6}\right): \delta 7.6\left[\mathrm{~N}\left(\mathrm{Si}\left(\mathrm{CH}_{3}\right)_{3}\right)_{2}\right], \delta 15.2\left[\mathrm{~N}\left(\mathrm{CH}_{2} \mathrm{CH}_{3}\right)_{2}\right], \delta$ $49.3\left[\mathrm{~N}\left(\mathrm{CH}_{2} \mathrm{CH}_{3}\right)_{2}\right], \delta 211.8[\mathrm{NCN}]$. HRMS (EI) $\mathrm{m} / \mathrm{z}$ calcd for $\mathrm{C}_{15} \mathrm{H}_{38} \mathrm{~N}_{3} \mathrm{Si}_{2} \mathrm{Cu} \mathrm{M} \mathrm{M}^{+}$ 379.1900 , found 379.1866 .

$N, N, N$, $N$ '-tetraisopropylformamidinylidene copper hexamethyldisilazide (10): $\mathrm{N}, \mathrm{N}, \mathrm{N}^{\prime}, \mathrm{N}^{\prime}$-tetraisopropylformamidinium chloride $(0.569 \mathrm{~g}, 2.29 \mathrm{mmol})$ was suspended in $30 \mathrm{~mL}$ of THF and copper(I) chloride $(0.233 \mathrm{~g}, 2.35 \mathrm{mmol})$ was added. The suspension was stirred for $18 \mathrm{~h}$. A light pink solid was collected by filtration, washed with $3 \times 2 \mathrm{~mL}$ of THF and then dried under vacuum. (This solid was presumed to be of equal ratio of the formamidinium chloride and copper(I) chloride). The pink solid ( $0.645 \mathrm{~g}, 1.85 \mathrm{mmol})$ was suspended in $30 \mathrm{~mL}$ of toluene and cooled to $-35^{\circ} \mathrm{C}$. $\mathrm{Et}_{2} \mathrm{O} \cdot \mathrm{LiN}\left(\mathrm{SiMe}_{3}\right)_{2}(0.895 \mathrm{~g}$, $3.71 \mathrm{mmol})$ was dissolved in $20 \mathrm{~mL}$ solution of toluene and diethyl ether (1:1) and added drop wise. The resultant mixture was allowed to warm to room temperature and was stirred for $18 \mathrm{~h}$. The cloudy solution was filtered and the insoluble fraction was washed with $2 \times 2 \mathrm{~mL}$ of toluene. The clear filtrate was combined with the washings and concentrated under vacuum to $15 \mathrm{~mL}$. The solution was chilled to $-35^{\circ} \mathrm{C}$ for 4 days to 
allow for the crystallization of a colourless solid. The solid was collected by decanting the solution and was dried under vacuum $(0.598 \mathrm{~g}, 59.9 \%) .{ }^{1} \mathrm{H}$ NMR $\left(400 \mathrm{MHz}, \mathrm{C}_{6} \mathrm{D}_{6}\right)$ : $\delta 0.54\left[\mathrm{~s}, 18 \mathrm{H}, \mathrm{N}\left(\mathrm{Si}\left(\mathrm{CH}_{3}\right)_{3}\right)_{2}\right], \delta 1.20\left[\mathrm{~d}, 24 \mathrm{H}, \mathrm{N}\left(\mathrm{CH}\left(\mathrm{CH}_{3}\right)_{2}\right)_{2}\right], \delta 3.28$ [sept, 4H, $\left.\mathrm{N}\left(\mathrm{CH}\left(\mathrm{CH}_{3}\right)_{2}\right)_{2}\right] .{ }^{13} \mathrm{C} \mathrm{NMR}\left(100, \mathrm{MHz}, \mathrm{C}_{6} \mathrm{D}_{6}\right): \delta 6.8\left[\mathrm{~N}\left(\mathrm{Si}\left(\mathrm{CH}_{3}\right)_{3}\right)_{2}\right], \delta 24.0$ $\left[\mathrm{N}\left(\mathrm{CH}\left(\mathrm{CH}_{3}\right)_{2}\right)_{2}\right], \delta 51.7\left[\mathrm{~N}\left(\mathrm{CH}\left(\mathrm{CH}_{3}\right)_{2}\right)_{2}\right], \delta 220.9[\mathrm{NCN}] . \mathrm{HRMS}(\mathrm{EI}) \mathrm{m} / \mathrm{z}$ calcd for $\mathrm{C}_{19} \mathrm{H}_{46} \mathrm{~N}_{3} \mathrm{Si}_{2} \mathrm{Cu} \mathrm{M} \mathrm{M}^{+} 435.2526$, found 435.2544.

\section{4,5-dimethyl-1,3-ditertbutyl-imidazolinium chloride: Cooled $54 \mathrm{~mL}$ of $1.6 \mathrm{M}$}

MeLi in $80 \mathrm{~mL}$ of $\mathrm{Et}_{2} \mathrm{O}$ to $0^{\circ} \mathrm{C}$ under a $\mathrm{N}_{2}$ atmosphere. $\mathrm{N}, \mathrm{N}^{\prime}$-ditertbutylethylenediimine ( $7.28 \mathrm{~g}, 43.3 \mathrm{mmol}$ ) was added in seven portions separated by 10 minutes. The solution was allowed to warm to RT and stirring proceded for $3 \mathrm{~h}$. The solution was re-cooled to 0 ${ }^{\circ} \mathrm{C}$ and $40 \mathrm{~mL}$ of distilled $\mathrm{H}_{2} \mathrm{O}$ was added dropwise and stirred for $12 \mathrm{~h}$. The organic layer was decanted and the aqueous layer was extracted with $2 \times 40 \mathrm{~mL}$ of $\mathrm{Et}_{2} \mathrm{O}$. The organic layers were combined and washed with $20 \mathrm{ml}$ of distilled $\mathrm{H}_{2} \mathrm{O}$. Paraformaldehyde $(1.455 \mathrm{~g}, 48.8 \mathrm{mmol})$ was added to the $\mathrm{Et}_{2} \mathrm{O}$ solution and stirred for $24 \mathrm{~h}$. The reaction solution was dried over $\mathrm{MgSO}_{4}$ and volatiles were removed under reduced pressure to afford the presummed 4,5-dimethyl-1,3-ditertbutylimidazolidine $(8.79 \mathrm{~g}, 41.4 \mathrm{mmol}$, 95.7\%). 4,5-dimethyl-1,3-ditertbutylimidazolidine (5.000 g, $23.5 \mathrm{mmol})$ was dissolved in $100 \mathrm{ml}$ of dimethoxyethane and cooled to $0{ }^{\circ} \mathrm{C}$. N-chlorosuccinimide $(3.144 \mathrm{~g}, 23.5$ mmol) was added portionwise afterwhich the solution was stirred overnight at RT. Volatiles were stripped off under reduced pressure and the succinimide by-product was removed by sublimation $\left(130^{\circ} \mathrm{C}, 30\right.$ mtorr) to afford the crude product as a brown solid (3.673 g, $63.2 \%) .{ }^{1} \mathrm{H} \mathrm{NMR}\left(400 \mathrm{MHz}, \mathrm{CDCl}_{3}\right): \delta 1.38\left[\mathrm{~d}, 6 \mathrm{H} \mathrm{N}\left(\mathrm{CHCH}_{3}\right)_{2} \mathrm{~N}\right], \delta 1.61[\mathrm{~s}$, $\left.18 \mathrm{H}, \mathrm{NC}\left(\mathrm{CH}_{3}\right)_{3}\right], \delta 3.81\left[\mathrm{q}, 2 \mathrm{H}, \mathrm{N}\left(\mathrm{CHCH}_{3}\right)_{2} \mathrm{~N}\right], \delta 9.54[\mathrm{~s}, 1 \mathrm{H}, \mathrm{NCHN}] \cdot{ }^{13} \mathrm{C} \mathrm{NMR}(100$ 
$\left.\mathrm{MHz}, \mathrm{CDCl}_{3}\right): \delta 21.0\left[\mathrm{~N}\left(\mathrm{CHCH}_{3}\right)_{2} \mathrm{~N}\right], \delta 30.1\left[\mathrm{NC}\left(\mathrm{CH}_{3}\right)_{3}\right], \delta 57.7\left[\mathrm{NC}\left(\mathrm{CH}_{3}\right)_{3}\right], \delta 62.9$ $\left[\mathrm{N}\left(\mathrm{CHCH}_{3}\right)_{2} \mathrm{~N}\right], \delta 154.5[\mathrm{NCHN}]$.

1,3-diethyl-imidazolinium chloride: N,N'-diethylethylenediamine (4.55 g, 39.2 mmol) was dissolved in $100 \mathrm{~mL}$ of triethylorthoformate under a nitrogen atmosphere. The solution was cooled to $0{ }^{\circ} \mathrm{C}$ and $4 \mathrm{M} \mathrm{HCl}$ in 1,4-dioxane $(19.6 \mathrm{~mL}, 78.3 \mathrm{mmol})$ was added dropwise. A volumous white precipitate formed and the reaction mixture was stirred for $1 \mathrm{~h}$. Two drops of formic acid where added the mixture was refluxed under $\mathrm{N}_{2}$ at $120{ }^{\circ} \mathrm{C}$ for $24 \mathrm{~h}$. The clear reaction solution was cooled and $400 \mathrm{~mL}$ of heptane was added to separate the reaction mixture into two layers. The top layer was discarded and the bottom layer was stripped of volatiles under reduced pressure and dried overnight under vacuum to afford a light brown solid (4.367 g, $71 \%$ ). ${ }^{1} \mathrm{H}$ NMR $\left(400 \mathrm{MHz}, \mathrm{CDCl}_{3}\right)$ : $\delta 1.36\left[\mathrm{t}, 6 \mathrm{H}, \mathrm{NCH}_{2} \mathrm{CH}_{3}\right], \delta 3.72\left[\mathrm{q}, 4 \mathrm{H}, \mathrm{NCH}_{2} \mathrm{CH}_{3}\right], \delta 4.01\left[\mathrm{~s}, 4 \mathrm{H}, \mathrm{N}\left(\mathrm{CH}_{2}\right)_{2} \mathrm{~N}\right], \delta 10.09$ [s, $1 \mathrm{H}, \mathrm{NCHN}] .{ }^{13} \mathrm{C} \mathrm{NMR}\left(100 \mathrm{MHz}, \mathrm{CDCl}_{3}\right): \delta 13.0\left[\mathrm{NCH}_{2} \mathrm{CH}_{3}\right], \delta 43.1\left[\mathrm{NCH}_{2} \mathrm{CH}_{3}\right], \delta$ $47.8\left[\mathrm{~N}\left(\mathrm{CH}_{2}\right)_{2} \mathrm{~N}\right], \delta 158.0[\mathrm{NCHN}]$. $N, N, N$ ',N'-tetraethylformamidinium chloride: $\mathrm{N}, \mathrm{N}$-diethylformamide $(7.36 \mathrm{~mL}$, $66.1 \mathrm{mmol})$ and diethylcarbamoyl chloride $(23.24 \mathrm{~mL}, 183.4 \mathrm{mmol})$ were added to a pressure vessel and backflushed with $\mathrm{N}_{2}$. The pressure vessel was heated to $145^{\circ} \mathrm{C}$ in an oil bath for $48 \mathrm{~h}$. The pressure vessel was removed from the oil bath. The brown solution solidified as the pressure vessel cooled to RT. The solid was washed with $3 \times 30 \mathrm{~mL}$ of $\mathrm{Et}_{2} \mathrm{O}$ and dried under vacuum to afford a light brown solid (11.305 g, $\left.75.4 \%\right) .{ }^{1} \mathrm{H}$ NMR (400 MHz, $\left.\mathrm{CDCl}_{3}\right): \delta 1.34$ [br s, $\left.12 \mathrm{H}, \mathrm{N}\left(\mathrm{CH}_{2} \mathrm{CH}_{3}\right)_{2}\right], \delta 3.48\left[\mathrm{br} \mathrm{s}, 4 \mathrm{H}, \mathrm{N}\left(\mathrm{CH}_{2} \mathrm{CH}_{3}\right)_{2}\right], \delta$ $3.85\left[\right.$ br s, $\left.4 \mathrm{H}, \mathrm{N}\left(\mathrm{CH}_{2} \mathrm{CH}_{3}\right)_{2}\right], \delta 9.49[\mathrm{~s}, 1 \mathrm{H}, \mathrm{NCHN}] .{ }^{13} \mathrm{C} \mathrm{NMR}\left(100 \mathrm{MHz}, \mathrm{CDCl}_{3}\right): \delta$ 
$14.4\left[\mathrm{~N}\left(\mathrm{CH}_{2} \mathrm{CH}_{3}\right)_{2}\right], \delta 14.7\left[\mathrm{~N}^{\prime}\left(\mathrm{CH}_{2} \mathrm{CH}_{3}\right)_{2}\right], \delta 43.0\left[\mathrm{~N}\left(\mathrm{CH}_{2} \mathrm{CH}_{3}\right)_{2}\right], \delta 51.6\left[\mathrm{~N}^{\prime}\left(\mathrm{CH}_{2} \mathrm{CH}_{3}\right)_{2}\right]$, $\delta 155.8[\mathrm{NCHN}]$.

Crystallography: Crystallographic studies for 2, 7, 8, and 10: Crystals were mounted using viscous oil onto a plastic mesh and cooled to the data collection temperature. Data were collected on a Bruker-AXS APEX CCD diffractometer with graphite-monochromated Mo-K $\alpha$ radiation $(\lambda=0.71073 \AA)$. Unit cell parameters were obtained from 36 data frames, $0.3^{\circ} \omega$, from three different sections of the Ewald sphere. No symmetry higher than triclinic was observed in $\mathbf{2}$ and $\mathbf{8}$. The systematic absences in the diffraction data are consistent with $C c$ and $C 2 / c$ for $\mathbf{1 0}$ and, uniquely, with $P 2_{1} / n$ for 7. For $\mathbf{8}$, and $\mathbf{1 0}$, solution in the centrosymmetric space group option yielded chemically reasonable and computationally stable results of refinement. The data-sets were treated with absorption corrections based on redundant multiscan data. The structures were solved using direct methods and refined with full-matrix, least-squares procedures on $F^{2}$. To compensate for imprecision introduced by molecular disorder on the crystallographic two-fold axis, the C-N distances in $\mathbf{1 0}$ were constrained to a common yet refined value. All non-hydrogen atoms were refined with anisotropic displacement parameters. All hydrogen atoms were treated as idealized contributions. Scattering factors are contained in several versions of the SHELXTL program library. ${ }^{56}$ The CIFs have been deposited under CCDC 1021530 - 1201532 and 1021719. 


\subsection{Supporting Information}

Table 7.3 Crystal Data and Structure Refinement Parameters.

\begin{tabular}{lllll}
\hline & $\mathbf{2}$ & $\mathbf{7}$ & $\mathbf{8}$ & $\mathbf{1 0}$ \\
\hline formula & $\mathrm{C}_{17} \mathrm{H}_{38} \mathrm{CuN}_{3} \mathrm{Si}_{2}$ & $\mathrm{C}_{19} \mathrm{H}_{44} \mathrm{CuN}_{3} \mathrm{Si}_{2}$ & $\mathrm{C}_{11} \mathrm{H}_{30} \mathrm{CuN}_{3} \mathrm{Si}_{2}$ & $\mathrm{C}_{19} \mathrm{H}_{46} \mathrm{CuN}_{3} \mathrm{Si}_{2}$ \\
formula weight & 404.23 & 434.30 & 324.10 & 436.31 \\
crystal system & triclinic & monoclinic & triclinic & monoclinic \\
space group & $\mathrm{P}-1$ & $\mathrm{P} 2{ }_{1} / \mathrm{n}$ & $\mathrm{P}-1$ & $\mathrm{C} 2 / \mathrm{c}$ \\
$\mathrm{a}(\AA)$ & $8.928(2)$ & $13.876(6)$ & $8.6287(5)$ & $15.3322(5)$ \\
$\mathrm{b}(\AA)$ & $9.911(3)$ & $10.287(4)$ & $9.6320(5)$ & $12.6279(4)$ \\
$\mathrm{c}(\AA)$ & $14.638(4)$ & $18.863(8)$ & $11.7892(6)$ & $14.4925(5)$ \\
$\alpha\left({ }^{\circ}\right)$ & $107.129(4)$ & 90.00 & $80.207(2)$ & 90.00 \\
$\beta\left({ }^{\circ}\right)$ & $101.712(4)$ & $106.086(8)$ & $82.472(2)$ & $107.756(1)$ \\
$\gamma\left({ }^{\circ}\right)$ & $101.579(4)$ & 90.00 & $67.289(2)$ & 90.00 \\
$\mathrm{~V}\left(\AA^{3}\right)$ & $1164.1(5)$ & $2587.1(18)$ & $888.15(8)$ & $2672.28(15)$ \\
$\mathrm{Z}$ & 2 & 4 & 2 & 4 \\
$\rho\left(\mathrm{g} / \mathrm{cm}^{3}\right)$ & 1.153 & 1.115 & 1.212 & 1.084 \\
goodness of fit & 1.035 & 1.007 & 1.050 & 1.014 \\
$\mathrm{R}\left(\mathrm{F}_{\mathrm{o}}\right)$ & 0.0333 & 0.0415 & 0.0204 & 0.0212 \\
$\mathrm{wR}\left(\mathrm{F}_{\mathrm{o}}\right)$ & 0.0935 & 0.0980 & 0.0562 & 0.0556 \\
\hline
\end{tabular}




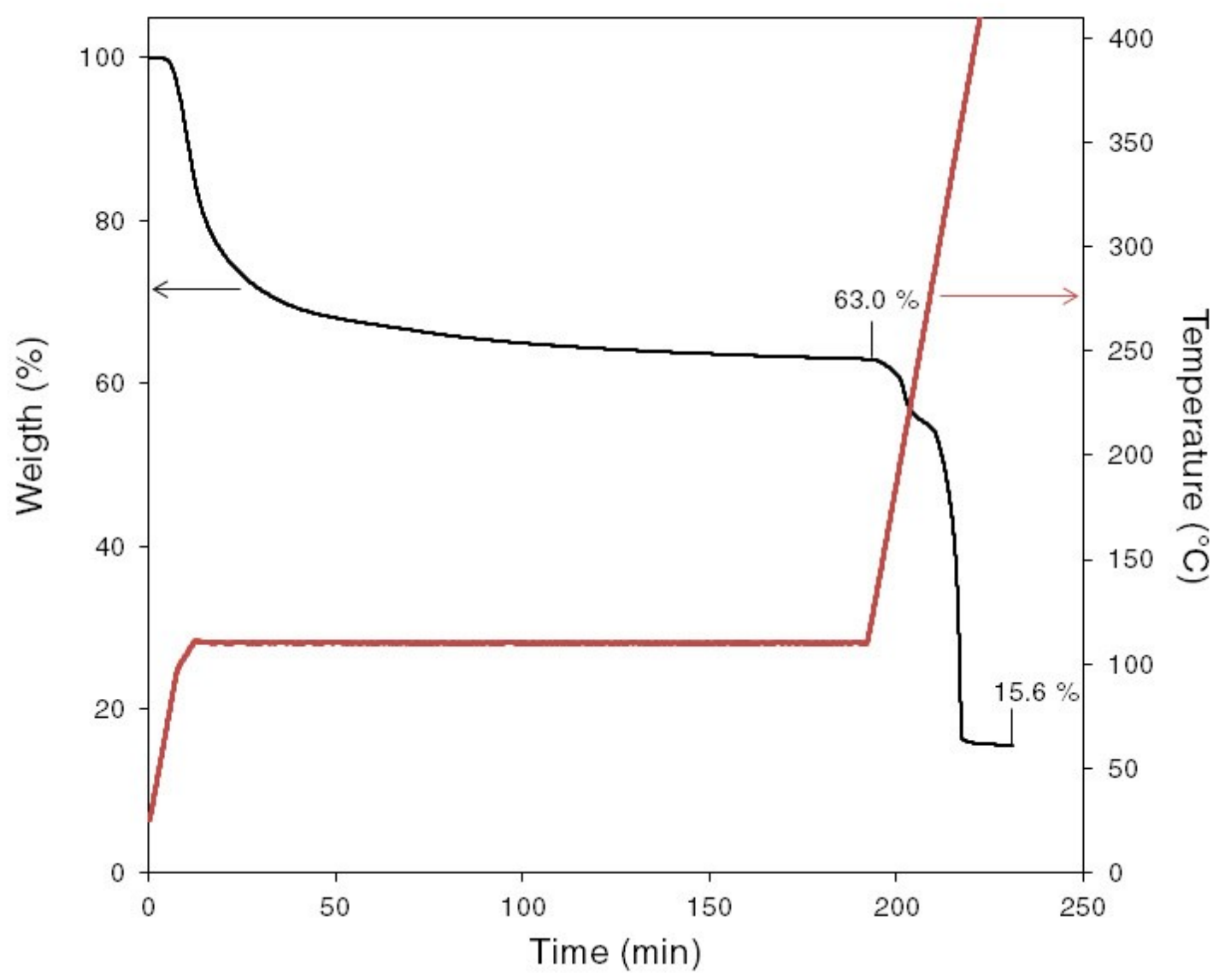

Figure 7.10 Isothermal TGA experiment of compound 2. An isotherm at $110^{\circ} \mathrm{C}$ was maintained until mass loss was negligible, followed by heating at a rate of $10^{\circ} \mathrm{C} / \mathrm{min}$. 


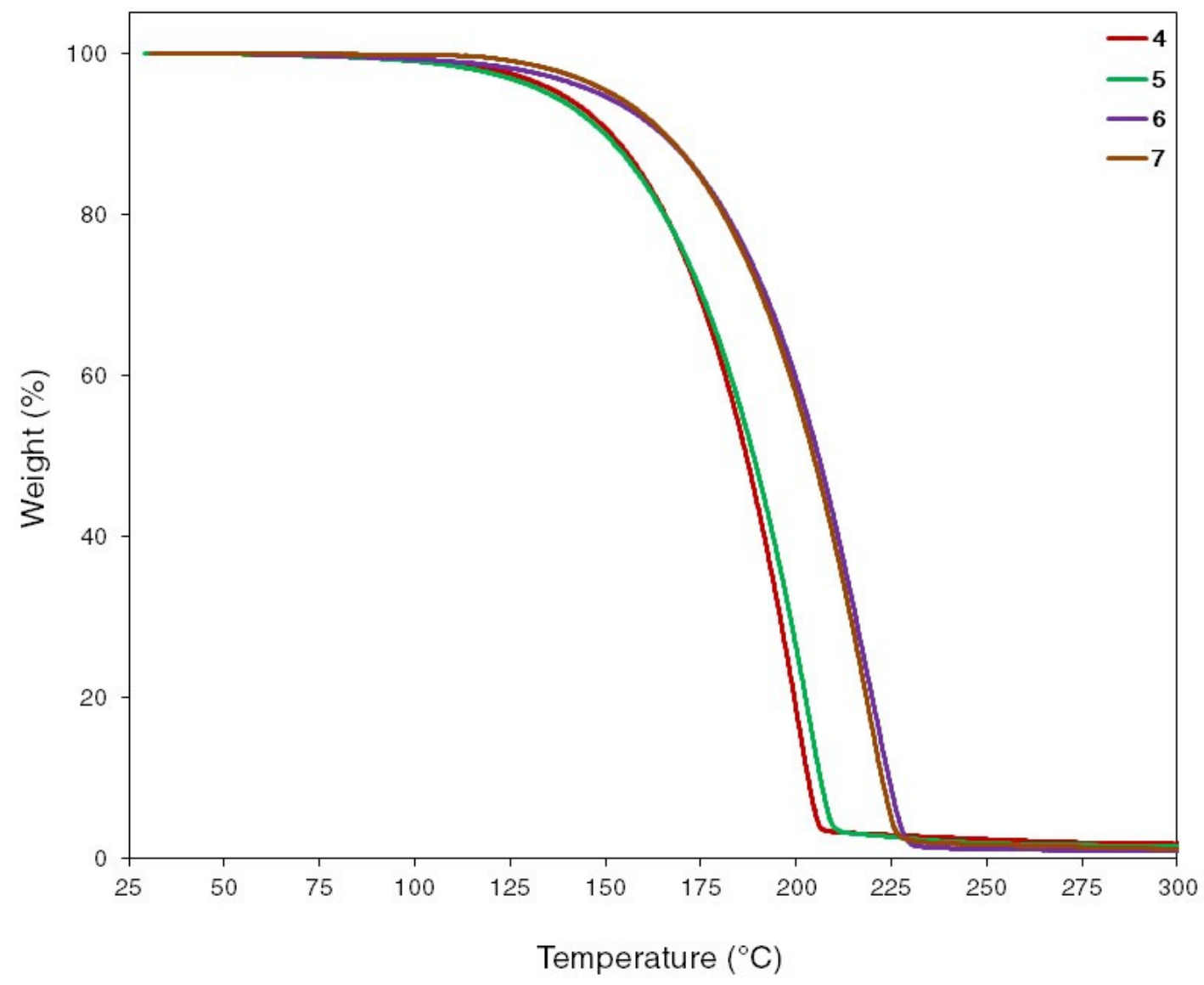

Figure 7.11 TGA experiments for the imidazolinylidene $\mathrm{Cu}(\mathrm{I})$ HMDS compounds 4 - 7 under $1 \mathrm{~atm} \mathrm{~N}_{2}$. The heating rate was $10^{\circ} \mathrm{C} / \mathrm{min}$ and sample masses were $4-6 \mathrm{mg}$. 


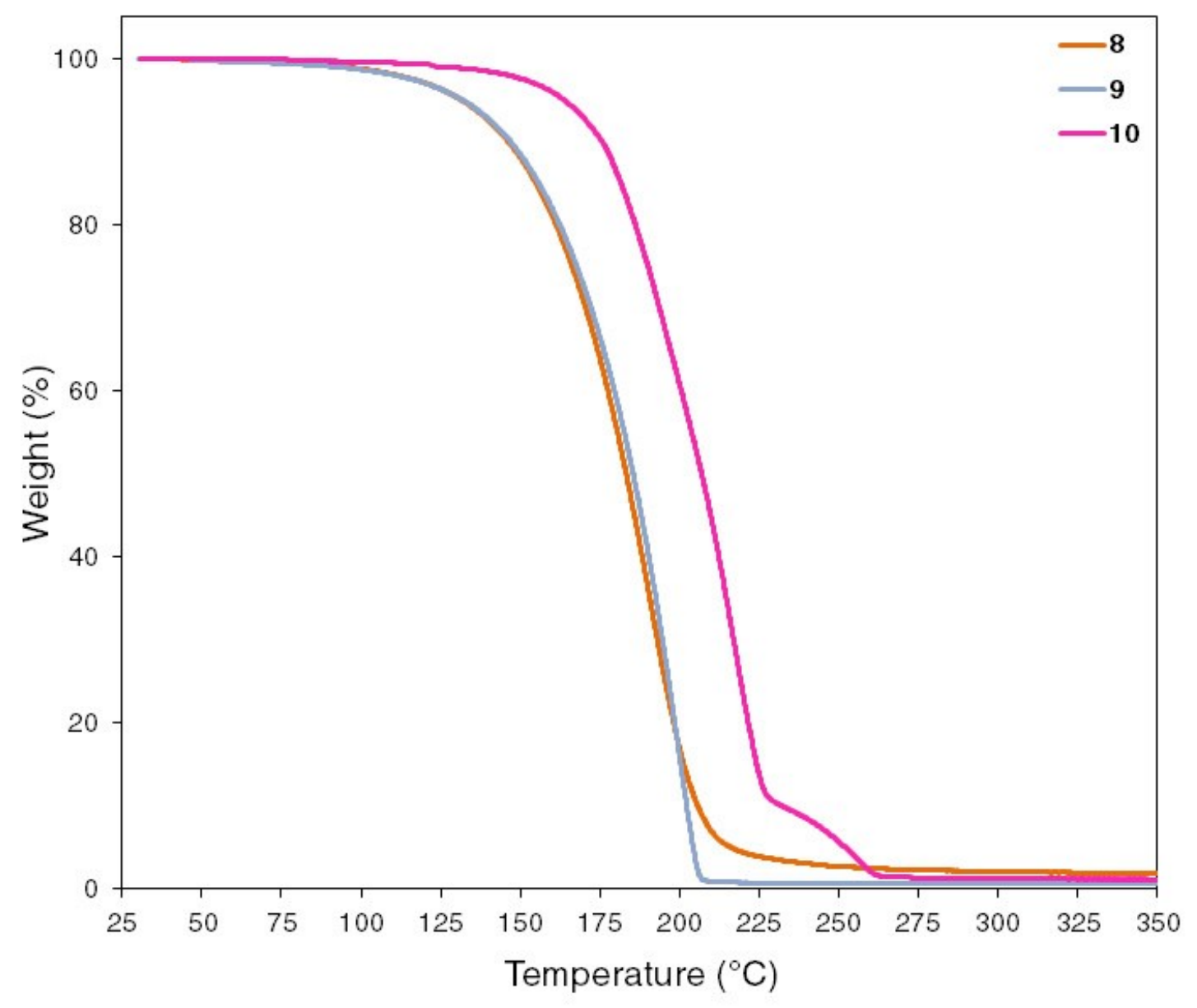

Figure 7.12 TGA experiments for the formamidinylidene $\mathrm{Cu}(\mathrm{I})$ HMDS compounds 8 10 under $1 \mathrm{~atm} \mathrm{~N}_{2}$. The heating rate was $10^{\circ} \mathrm{C} / \mathrm{min}$ and sample masses were $4-6 \mathrm{mg}$. 


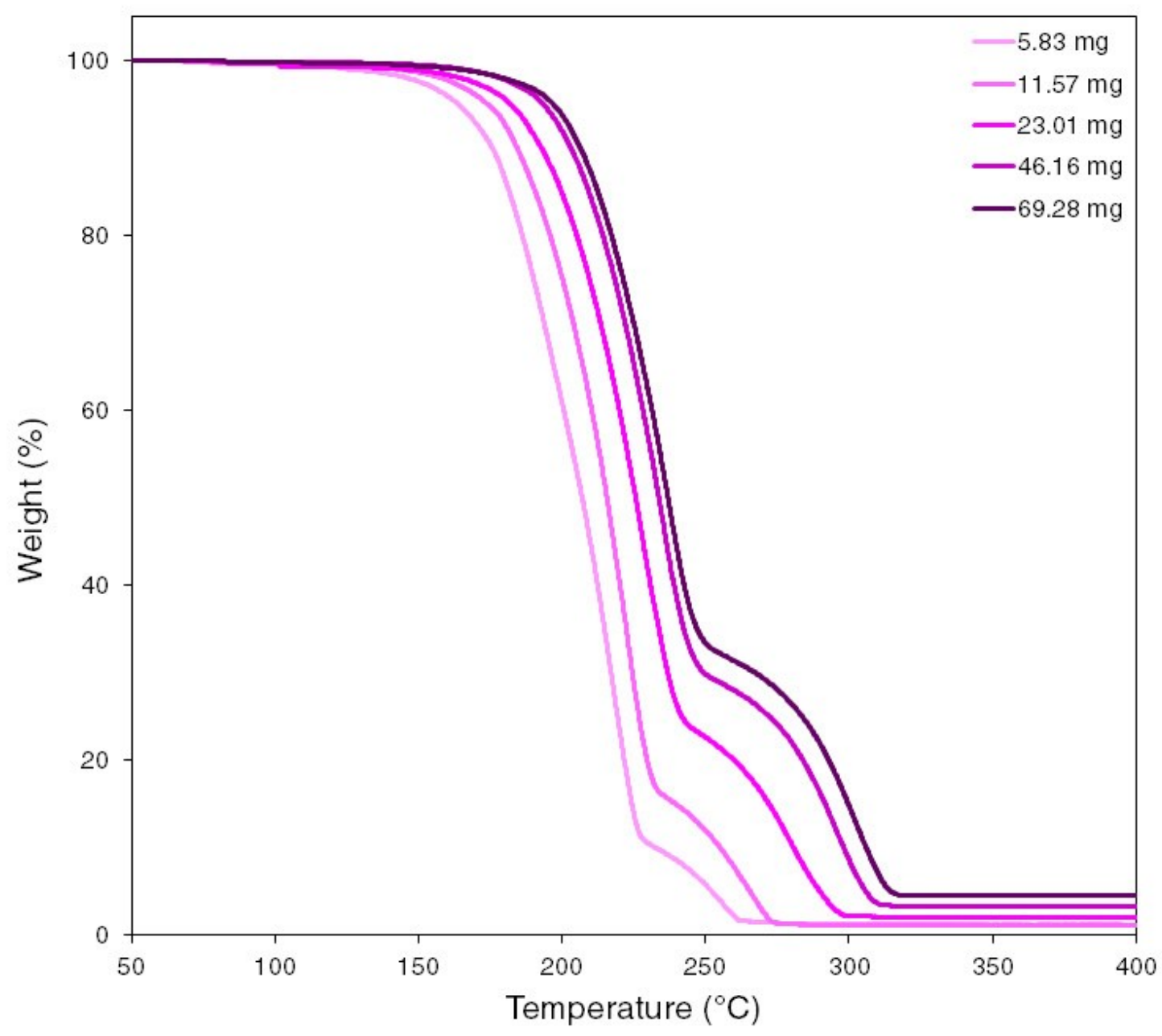

Figure 7.13 TGA thermal stress test for compound $\mathbf{1 0}$ illustrating thermal decomposition. The heating rate was $10{ }^{\circ} \mathrm{C} / \mathrm{min}$ and sample masses are shown top right. 


\subsection{References}

1 International Technology Roadmap for Semiconductors; 2013; http://www.itrs.net/.

2 (a) Chen, T. Y.; Omnes, L.; Vaisserman, J.; Doppelt, P. Inorg. Chim. Acta. 2004, 357, 1299, (b) Bollmann, D.; Merkel, R.; Klumpp, A. Microelectron. Eng. 1997, 37-8, 105, (c) Lagalante, A. F.; Hansen, B. N.; Bruno, T. J.; Sievers, R. E. Inorg. Chem. 1995, 34, 5781 (d) Wenzel, T. J.; Williams, E. J.; Haltiwanger, R. C.;

Sievers, R. E. Polyhedron 1985, 4, 369.

3 Park, K. H.; Marshall, W. J. J. Am. Chem. Soc. 2005, 127, 9330.

4 (a) Li, Z. W.; Rahtu, A.; Gordon, R. G. J. Electrochem. Soc. 2006, 153, C787, (b) Li, Z. W.; Barry, S. T.; Gordon, R. G. Inorg. Chem. 2005, 44, 1728, (c) Lim, B. S.; Rahtu, A.; Gordon, R. G. Nat. Mater. 2003, 2, 749, (d) Lim, B. S.; Rahtu, A.; Park, J. S.; Gordon, R. G. Inorg. Chem. 2003, 42, 7951.

5 Coyle, J. P.; Monillas, W. H.; Yap, G. P. A.; Barry, S. T. Inorg. Chem. 2008, 47, 683.

6 Park, J. W.; Jang, H. S.; Kim, M.; Sung, K.; Lee, S. S.; Chung, T.; Koo, S.; Kim, C. G.; Kim, Y. Inorg. Chem. Commun. 2004, 7, 463.

7 Grushin, V. V.; Marshall, W. J. Adv. Synth. Catal. 2004, 346, 1457.

8 Coyle, J. P.; Dey, G.; Sirianni, E. R.; Kemell, M. L.; Yap, G. P. A.; Ritala, M.; Leskelä, M. Elliot, S. D.; Barry, S. T. Chem. Mater. 2013, 25, 1132.

9 Gambarotta, S.; Bracci, M.; Floriani, C.; Chiesi-Villa, A.; Guastini, C. J. Chem. Soc. Dalton Trans. 1987, 1883.

10 Dey, G.; Elliott, S. D. Theor. Chem. Acc. 2014, 133, 1416.

11 Hopkinson, M. N.; Richter, C.; Schedler, M.; Glorius, F. Nature 2014, 510, 485.

12 (a) Dröge, T.; Glorius, F. Angew. Chem. Int. Ed. 2010, 49, 6940, (b) Kumar, A.; Ghosh, P. Eur. J. Inorg. Chem. 2012, 3955.

13 Praetorius, J. M.; Crudden, C. M. RSC Catalysis Series, 2011, 6, 77.

14 Kuhn, K. M.; Bourg, J.-B.; Chung, C. K.; Virgil, S. C.; Grubbs, R. H. J. Am. Chem. Soc., 2009, 131, 5313. 
15 Lin, J. C. Y.; Huang, R. T. W.; Lee, C. S.; Bhattacharyya, A.; Hwang, W. S.; Lin, I. J. B. Chem. Rev. 2009, 109, 3561.

16 Egbert, J. D.; Cazin, C. S.; Nolan, S. P. Catal. Sci. Technol. 2013, 3, 912.

17 Nemcsok, D.; Wichmann, K.; Frenking, G. Organometallics 2004, 23, 3640.

18 Díez-González, S.; Escudero-Adán, E. C.; Benet-Buchholz, J.; Stevens, E. D.; Slawin, A. M. Z.; Nolan, S. P. Dalton Trans., 2010, 39, 7595.

19 Hahn, F. E.; Jahnke, M. C. Angew. Chem. Int. Ed. 2008, 47, 3122.

20 (a) Alder, R. W.; Allen, P. R.; Murray, M.; Orpen, A. G. Angew. Chem. Int. Ed. 1996, 35, 1121. (b) Alder, R. W.; Blake, M. E.; Chaker, L.; Harvey, J. N.; Paolini, F.; Schutz, J. Angew. Chem. Int. Ed. 2004, 43, 5896. (c) Alder, R. W.; Blake, M. E.; Bufali, S.; Butts, C. P.; Orpen, A. G.; Schütz, J.; Williams, S. J. J. Chem. Soc., Perkin Trans. 1. 2001, 1586.

21 The carbene salts and copper chloride are insoluble in THF on their own, but dissolved when mixed together presumably forming a cuprate ion pair, $\left[\mathrm{NHC} \cdot \mathrm{H}^{+}\right]\left[\mathrm{CuCl}_{2}{ }^{-}\right]$. For example: Blue, E. D.; Gunnoe, T. B.; Petersen, J. L.; Boyle, P. D. J. Organomet. Chem. 2006, 691, 5988 and ref. 22d.

22 (a) Slivarichova, M.; Ahmad, R.; Kuo, Y.-Y.; Nunn, J.; Haddow, M. F.; Othman, H.; Owen, G. R. Organometallics 2011, 30, 4779. (b) Denk, M. K.; Rodezno, J. M.; Gupta, S.; Lough, A. J. J. Organomet. Chem. 2001, 617-618, 242 (c) Citadelle. C. A.; Le Nouy, E.; Bisaro, F.; Slawin, A. M. Z.; Cazin, C. S. J. Dalton Trans. 2010, 39, 4489. (d) Santoro, O.; Collado, A.; Slawin, A. M. Z.; Nolan, S. P.; Cazin, C. S. J. Chem. Comm.2013, 49, 10483.

23 James, A. M.; Laxman, R. K.; Fronczek, F. R.; Maverick, A. W. Inorg. Chem. 1998, 37, 3785

24 Li W.; Hill, J. N.; Tomasik, A. C.; Bikzhanova, G.; West, R. Organometallics 2006, 25,3802

Mayr, M.; Wurst, K.; Ongania, K.-H.; Buchmeiser, M. R. Chem. Eur. J. 2004, 10, 1256.

$26 \mathrm{SI}^{\mathrm{t}} \mathrm{Bu}_{2} \mathrm{Me}_{2} \cdot \mathrm{HCl}$ was treated with 1 equiv. of LiHMDS in THF. $\mathrm{SI}^{\mathrm{t}} \mathrm{Bu}_{2} \mathrm{Me}_{2}$ was extracted with pentane from the dry reaction solid after removal of volatile materials in vacuo. ${ }^{1} \mathrm{H}$ NMR $\left(400 \mathrm{MHz}, \mathrm{C}_{6} \mathrm{D}_{6}\right): \delta 0.91[\mathrm{~d}, 6 \mathrm{H}], \delta 1.43[\mathrm{~s}, 18 \mathrm{H}], \delta$ $3.07[\mathrm{q}, 2 \mathrm{H}] .{ }^{13} \mathrm{C}$ NMR $\left(100 \mathrm{MHz}, \mathrm{C}_{6} \mathrm{D}_{6}\right): \delta 22.8, \delta 31.8, \delta 54.0, \delta 62.7, \delta 238.6$. 
27 West, J. K.; Fondong, G. L.; Noll, B. C.; Stahl, L. Dalton Trans. 2013, 42, 3835.

28 (a) Tapu, D.; Dixon, D. A.; Roe, C. Chem. Rev. 2009, 109, 3385. (b) Nelson, D. J.; Nolan, S. P. Chem. Soc. Rev. 2013, 42, 6723. (c) Huynh, H. V.; Han, Y.; Jothibasu, R.; Yang, J. A. Organometallics 2009, 28, 5395. (d) Frey, G. D.; Herdtweck, E.; Herrmann, W. A. J. Organomet. Chem. 2006, 691, 2465

29 Back, O.; Henry-Ellinger, M.; Martin, C. D.; Martin, D.; Bertrand, G. Angew. Chem. Int. Ed. 2013, 52, 2939.

30 (a) Jacobsen, H.; Correa, A.; Poater, A.; Costabile, C.; Luigi, C. Coord. Chem. Rev. 2009, 253, 687. (b) Marchione, D.; Belpassi, L.; Bistoni, G.; Macchioni, A.; Tarantelli, F.; Zuccaccia, D. Organometallics 2014, 33, 4200.

31 Hu, X.; Castro-Rodriguez, I.; Olsen, K.; Meyer, K. Organometallics 2004, 23, 755.

32 Alcarazo, M.; Stork, T.; Anoop, A.; Thiel, W.; Fürstner, A. Angew. Chem. Int. Ed. 2010, 49, 2542.

33 Poater, A.; Cosenza, B.; Correa, A.; Giudice, S.; Ragone, F.; Scarano, V.; Cavallo, L. Eur. J. Inorg. Chem. 2009, 1759.

34 Clavier, H.; Nolan, S. P. Chem. Comm. 2010, 46, 841.

$35{ }^{1} \mathrm{H}$ NMR analysis found two singlets at $1.79 \mathrm{ppm}$ and $7.05 \mathrm{ppm}$ which integrated to a 1:9 ratio. ${ }^{13} \mathrm{C}$ NMR analysis found peaks at $32.1 \mathrm{ppm}, 57.7 \mathrm{ppm}$ and $116.5 \mathrm{ppm}$. These chemical shifts are an exact match to those reported for $\left[\mathrm{I}^{\mathrm{t}} \mathrm{Bu}_{2}\right] \mathrm{CuCl}$ in ref. 18. The residual material from TGA was insoluble in most solvents. It dissolved slowly in $\mathrm{CDCl}_{3}$ presumably via reaction with residual $\mathrm{HCl}$.

36 Denk, M. K.; Rodezno, J. M. J. Organomet. Chem. 2000, 608, 122.

37 Dubinina, G. G.; Furutachi, H.; Vicic, D. A. J. Am. Soc. Chem. 2008, 130, 8600.

38 Wang, Y.; Xie, Y.; Abraham, M. Y.; Wei, P.; Schaefer, H. F. III; Schleyer, P. v. R.; Robinson, G. H. J. Am. Soc. Chem. 2010, 132, 14370.

39 (a) Caddick, S.; Cloke, F. G. N.; Hitchcock, P. B.; Lewis, A. K. de, K. Angew. Chem. Int. Ed. 2004, 43, 5824. (b) Burling, S.; Mahon, M. F.; Powell, R. E.; Whittlesey, M. K.; Williams, J. M. J. J. Am. Soc. Chem. 2006, 128, 13702. (c) Day, B. M.; Pugh, T.; Hendriks, D.; Guerra, C. F.; Evans, D. J.; Bickelhaupt, F. M.; Layfield, R. A. J. Am. Chem. Soc. 2013, 135, 13338. 
40 Coyle, J. P.; Pallister, P. J.; Kurek, A.; Sirianni, E. R.; Yap, G. P. A. Barry, S. T. Inorg. Chem. 2013, 52, 910.

41 Hillier, A. C.; Sommer, W. J.; Yong, B. S.; Petersen, J. L.; Cavallo, L.; Nolan, S. P. Organometallics 2003, 22, 4322.

42 Yi, W.; Zhang, J.; Chen, Z.; Zhou, X. Inorg. Chem. 2012, 51, 10631.

43 (a) Yu, X.; Bi, S.; Guzei, I. A.; Lin, Z.; Xue, Z.-L. Inorg. Chem. 2004, 43, 7111. (b) Korobkov, I.; Gambarotta, S. Inorg. Chem. 2010, 49, 3409.

44 Schulz, T.; Leibold, M.; Färber, C.; Maurer, M.; Porsch, T.; Holthausen, M. C.; Siemeling, U. Chem. Comm. 2012, 48, 9123.

45 Gillan, E. G.; Bott, S. C.; Barron, A. R. Chem. Mater. 1997, 9, 796.

46 Kunte, G. V.; Shivashankar, S. A.; Umarji, A. M. Meas. Sci. Technol. 2008, 19, 025704 .

47 The 1 Torr values for $\mathbf{3}$ and $\mathbf{5}$ are corrected from our previous publication (reference 8 ) because of a calculation error for the value of the sample pan surface area.

48 Waffenschmidt, E.; Musolf, J.; Heuken, M.; Heime, K. J. Supercond. 1992, 5, 119.

49 Igumenov, I. K.; Basova, T. V.; Belosludov, V. R. Volatile Precursors for Films Deposition: Vapor Pressure, Structure and Thermodynamics. In Application of Thermodynamics to Biological and Materials Science, Tadashi, M., Ed.; InTech: Rijeka, 2011, pp. 521-546.

50 (a) Johnson, M. G.; Selvakumar, J.; Nagaraja, K. S. Thermochimica Acta 2009, 495, 38. (b) Colominas, C.; Lau, K. H.; Hildenbrand, D. L.; Crouch-Baker, S.; Sanjurjo, A. J. Chem. Eng. Data 2001, 46, 446.

51 Arduengo, A. J., III. U.S., Patent 5182405, 1993.

52 Bittermann, A.; Baskakov, D.; Herrmann, W. A. Organometallics, 2009, 28, 5107.

53 Arentsen, K. Caddick, S. Cloke, F. G. N. Tetrahedron, 2005, 61, 9710.

54 Wasserman, H. H.; Ives, J. L. J. Org. Chem. 1985, 50, 3573.

55 Lappert, M. F.; Slade, M. J.; Singh, A.; Atwood, J. L.; Rogers, D. R.; Shakir, R. J. Am. Soc. Chem. 1983, 105, 302. 
56 Sheldrick, G.M. Acta Cryst. 2008, A64, 112-122 


\section{Chapter: Conclusions}

Two research programs pertaining to the development of precursors for the atomic layer deposition of copper metal thin films were carried out. Each program set out in a unique direction while ending, in each case, with new and advanced copper precursors. The first program built on the well-known copper(I) amidinate precursors. Several modifications to the amidinate framework were made and the effects on the thermal chemistry of the respective copper compounds were studied. By this approach, weaknesses of a ligand framework were identified and ways to prevent thermolysis routes could be explored. Copper(I) tert-butyl-imino-2,2-dimethylpyrrolidinate was discovered through this manner and it demonstrates a high level of thermal stability. The second program was initiated by experiments aimed at breaking the dimeric copper(I) amidinate structure with N-heterocyclic carbenes. Instead, a popular ligand in organometallic chemistry was introduced to precursor design and, consequently, the small void of copper(I) amide complexes in the library of copper ALD precursors was filled. This particular precursor formulation, i.e. $\mathrm{NHC}-\mathrm{Cu}-\mathrm{NR}_{2}$, was unknown and required optimization through a similar process responsible for the advancement of copper(I) amidinates to iminopyrrolidinates

Both research programs relied extensively on the synthesis of new compounds and characterisation by thermal gravimetric analysis to validate precursor concepts. Advanced gas-phase techniques were invaluable in determining between decomposition mechanisms that were not easily distinguished by bench-top experiments. Much of the precursor development proceeded without ALD experiments to validate or influence the design choices. The knowledge gained from this work allowed for many comparisons 
between the principle characteristics of the potential precursors, however; it was not determined if these precursor differences have a substantial effect on the characteristics of film growth during ALD, e.g. growth rate or ALD temperature window.

Copper(I) guanidinates were synthesized and characterised by thermal gravimetric analysis, solution decomposition studies and in-situ mass-spectrometry monitoring of gas-phase thermolysis products during chemical vapour deposition experiments. Results for the copper(I) guanidinates were compared to a copper(I) amidinate. A latter study reported two additional copper(I) guanidinates and completed a series of four compounds, $\left[\mathrm{Cu}\left({ }^{i} \operatorname{PrN}\right)_{2} \mathrm{CNR}{ }^{\prime} \mathrm{R}^{\prime}\right]_{2}$ where $\mathrm{R}^{\prime}=\mathrm{R} "=\mathrm{Me}, \mathrm{Et},{ }^{i} \operatorname{Pr}$ and $\mathrm{R}{ }^{\prime}=\mathrm{H}, \mathrm{R}{ }^{\prime}={ }^{i} \mathrm{Pr} .{ }^{1}$ The volatility of the copper(I) guanidinates compared well to that of the copper(I) amidinate. Noticeable differences were apparent in the geometry of their metallocycles and their observed decomposition products. Participation of the exocyclic nitrogen in the guanidinate ligand caused pyramidalization of the chelating nitrogens and twisting of the metallocycle core of the dimer. The degree of twisting was dictated by the alignment of the exocyclic lone pair with the delocalized $\pi$-system of the ligand which was influenced by the steric bulk of the exocyclic substituents. Evidence for the production of CDI during decomposition studies was found for the copper(I) guanidinates and not for the amidinate. The structural differences could be viewed as influential in the thermoylsis products since ligand flexibility and acrobatics would be required to deinsert a core fragment from the guanidinate ligand. Follow-up solution studies for two parent guanidines, ${ }^{\mathrm{i}} \mathrm{Pr}_{2} \mathrm{NC}\left(\mathrm{N}^{\mathrm{i}} \mathrm{Pr}\right)_{2} \mathrm{H}$ and $\mathrm{Me}_{2} \mathrm{NC}\left(\mathrm{N}^{\mathrm{i}} \mathrm{Pr}\right)_{2} \mathrm{H}$, revealed $\mathrm{CDI}$ deinsertion to occur under similar conditions used for the copper(I) guanidinates. ${ }^{1}$ Thus it is important to reconsider the origin of CDI and the possibility of accessible protic species or proton 
sources during the solution studies (e.g. dehydrogenation of the ligand, solvent impurities or the $\mathrm{SiO}_{2}$ surface of NMR tube). Reasonably, it would be worthwhile to revisit the solution studies with a copper(I) guanidinate containing an exocyclic nitrogen with substituents that would enable easy identification of the imine produced after CDI deinsertion, e.g. $\left[\mathrm{Cu}\left(\mathrm{N}^{i} \mathrm{Pr}\right)_{2} \mathrm{CN}^{n} \mathrm{Bu}_{2}\right]_{2}$.

CVD experiments for the copper(I) guanidinates demonstrated its utility as a single source precursor for pure copper metal thin films, similar to copper(I) diketonates precursors, albeit by a different surface mechanism. Future work for copper(I) guanidinates should focus on ALD experiments and investigate their temperature window for self-limited film growth in comparison to the copper(I) amidinates. Particular attention should be paid to low deposition temperatures, perhaps by PE-ALD, to determine if the twisted metallocycle assists in chemisorption at lower temperature. Thermal chemistry for $\left[\mathrm{Cu}\left(\mathrm{N}^{i} \mathrm{Pr}\right)_{2} \mathrm{CNMe}_{2}\right]_{2}$ on a $\mathrm{Ni}(110)$ single crystal under ultrahigh vacuum conditions have been reported, succeeding earlier reports for $\left[\mathrm{CuN}\left({ }^{s} \mathrm{Bu}\right)_{2} \mathrm{CMe}\right]_{2}$, and no evidence was obtained for CDI deinsertion. ${ }^{2}$

Gas-phase thermolysis products for $\left[\mathrm{Cu}\left(\mathrm{N}^{i} \mathrm{Pr}\right)_{2} \mathrm{CNMe}_{2}\right]_{2}$ were identified TOF MS and MI FTIR. These advanced techniques were capable of unambiguously identifying decomposition fragments due to the high resolution and detection limits of the mass spectrometer combined with the ability to corroborate with highly resolved infrared spectra. N,N-dimethyl-N'-iso-propyl-N"-iso-propylidene-guanidine, referred to as oxidized guanidine, was identified as a thermolysis product of $\left[\mathrm{Cu}\left(\mathrm{N}^{i} \mathrm{Pr}\right)_{2} \mathrm{CNMe}_{2}\right]_{2}$ above $150^{\circ} \mathrm{C}$. Deposition of copper inside the thermolysis oven and molecular hydrogen were also observed. A $\beta$-hydride mechanism was proposed and was determined to be operative 
up to temperatures of $700{ }^{\circ} \mathrm{C}$ in the gas phase. UHV surface studies of $\left[\mathrm{Cu}\left(\mathrm{N}^{i} \mathrm{Pr}\right)_{2} \mathrm{CNMe}_{2}\right]_{2}$ also identified oxidized guanidine as a product of decomposition. ${ }^{2}$

DFT calculations were performed to help rationalize the fact that carbodimide deinsertion was observed at lower temperatures in the solution phase, but $\beta$-hydrogen elimination is favored in higher temperature, gas phase reactions. DFT calculations at the B3LYP/6-31+G(d,p) level of theory employing dispersion-correcting potential showed that at $298 \mathrm{~K}$ production of oxidized guanidine $(\Delta \mathrm{G}=-24.4 \mathrm{kcal} / \mathrm{mol}$, Figure 3.5$)$ is thermodynamically favourable compared to CDI deinsetion $(\Delta \mathrm{G}=-11.7 \mathrm{kcal} / \mathrm{mol}$, Figure 3.1). At $533 \mathrm{~K}$, the two decomposition pathways were equally exergonic, $\Delta \mathrm{G}=-44.4$ $\mathrm{kcal} / \mathrm{mol}$. Although enthalpy corrections are temperature dependent, the CDI deinsertion pathway has a more significant entropic driving force. An extensive DFT study modeled the fragmentation steps for the two pathways through a common intermediate and found $\beta$-hydrogen elimination and CDI deinsertion to be endergonic and exergonic pathways, respectively. This result helped rationalize why two pathways were discovered experimentally, but does not clearly explain why only $\beta$-hydrogen elimination was observed in the gas phase experiments. Perhaps, an alternate pathway for $\beta$-hydrogen elimination is possible which provides a low energy detour to the common intermediate. ${ }^{3}$

Copper(I) iminopyrrolidinates were synthesised to test modifications to the amidinate ligand designed to block decomposition pathways. Tethering one N-substituent to the exocyclic position inhibits the CDI deinsertion pathway while replacing $\beta$ hydrogens with methyl groups prevents the $\beta$-hydride abstraction pathway. Several copper(I) iminopyrrolidinates showed lower volatility than $\left[\mathrm{Cu}\left(\mathrm{N}^{i} \mathrm{Pr}\right)_{2} \mathrm{CNMe}_{2}\right]_{2}$. In solution thermolysis experiments, comparable decompositions rates were measured for 
the iminopyrrolidinates compounds possessing $\beta$-hydrogens and for $\left[\mathrm{Cu}\left(\mathrm{N}^{i} \mathrm{Pr}\right)_{2} \mathrm{CNMe}_{2}\right]_{2}$ at $165{ }^{\circ} \mathrm{C}$. Copper(I) tert-butyl-imino-2,2-dimethylpyrrolidinate possesses no $\beta$ hydrogens and was stable indefinitely at $165^{\circ} \mathrm{C}$ in solution and during TGA.

Copper(I) tert-butyl-imino-2,2-dimethylpyrrolidinate required temperatures $>275^{\circ} \mathrm{C}$ to chemisorb on high surface area silica which occurred with simultaneous elimination of its tert-butyl group, presumably as isobutene. Future ALD experiments exploring its ALD temperature window will complete the list of advantages and disadvantages for these particular ligand modifications. For instance, due to the rigidity of the robust iminopyrrolidinate ligand, high temperatures may be necessary to evaporate and chemisorb the precursor which would be an overall disadvantage compared to other precursors.

Thermal chemistry for copper(I) N-sec-butyl-iminopyrrolidinate on a $\mathrm{Ni}(110)$ single crystal has been investigated and similar experiments are underway for copper(I) tert-butyl-imino-2,2-dimethylpyrrolidinate. ${ }^{2}$ The oxidized iminopyrrolidine, 2-secbutyleneamino-1-pyrroline, was observed as a gas phase product at $\sim 300 \mathrm{~K}$, thus $\beta$ hydride abstraction is expected to take place immediately upon precursor adsorption under film deposition conditions. The transition of adsorbed $\mathrm{Cu}(\mathrm{I})$ atoms to $\mathrm{Cu}(0)$ was observed at $350-400 \mathrm{~K}$ and desorption of smaller dehydrogenated ligand fragments were observed above $420 \mathrm{~K}$ indicating that not all adsorbed iminopyrrolidinate ligand undergoes $\beta$-hydrogen elimination. The remaining surface chemistry for the iminopyrrolidinate ligand is qualitatively similar to what was previously observed for $\left[\mathrm{Cu}\left(\mathrm{N}^{s} \mathrm{Bu}\right)_{2} \mathrm{CMe}\right]_{2}{ }^{4}$ - a core $\mathrm{C}-\mathrm{N}$ bond breaks and the remaining halves of the ligands undergo further dehydrogenation chemistry. The main difference for iminopyrrolidinates 
is that one half of the ligand is cyclic which implies that susceptible bonds are slightly constrained from interacting with the surface. Upcoming experiments for copper(I) tertbutyl-imino-2,2-dimethylpyrrolidinate will tell whether the methylated ring inhibits surface dehydrogenation reactions and increase the robustness of the adsorbed precursor. If so, it would be a worthwhile endeavor to explore various copper(I) alkyl-imino-2,2dimethylpyrrolidinate precursors to optimize precursor volatility while maintaining robust surface chemistry.

The success of copper(I) tert-butyl-imino-2,2-dimethylpyrrolidinate prompted the analogous silver(I) and gold(I) compounds to be synthesized and studied as CVD precursors for metal films. These two particular silver and gold compounds proved to be a vast improvement compared to previously reported amidinate and guanidinate compounds. ${ }^{1}$ The iminopyrrolidinate compounds could be evaporated and entrained into a deposition zone without decomposing. For comparison, to obtain thin gold films from $\left[\mathrm{Au}\left(\mathrm{N}^{i} \mathrm{Pr}\right)_{2} \mathrm{CNMe}_{2}\right]_{2}$ the substrate is placed directly over the solid precursor. ${ }^{5}$ The evaporation temperatures of the precursors were sufficiently separated from their onset of metal deposition that future ALD experiments should be attempted. Because of their low evaporation rates an appropriate precursor delivery system may be needed. Other silver(I) and gold(I) alkyl-imino-2,2-dimethylpyrrolidinate compounds should be studied to discover an alternate precursor with higher rates of evaporation and higher solubility to simplify their synthesis.

Various diamino carbenes were tested as ligands for precursors in monomeric copper(I) hexamethyldisiliazide compounds. The carbenes investigated a range of Nsubstituents and nature of the carbon backbone. Many potential precursors were 
discovered. The series of imidazolinylidene compounds demonstrated consistent volality and thermal stability. Results for the formamidinylidene compounds were also encouraging. Since a good understanding has been established regarding the behavior and properties of a range of diamino carbenes it would be appropriate to expand the family of precursors stabilized by carbenes to include other copper(I) compounds; e.g. alkoxides, alkyl, alkylamido. A primary goal should be to reduce the steric bulk of the anionic ligand without sacrificing stability in order to assist the reactivity of the precursor with surfaces and reducing agents.

Initial ALD experiments employed 1,3-diisopropyl-imidazolin-2-ylidene copper hexamethyldisilazide in a plasma-enhanced process. Self limited growth was demonstrated at $225^{\circ} \mathrm{C}$ on silicon with a native oxide. Thermal ALD experiments involving the same precursor and molecular hydrogen were reported on Ru and Pd substrates. ${ }^{6}$ On Ru substrates isolated nanoparticles of copper metal were deposited between $170-240{ }^{\circ} \mathrm{C}$ with 1000 cycles. The density and size of the nanoparticles varied at each temperature which indicates a variation in either precursor chemisorption or reactivity of molecular hydrogen. On Pd substrates, film continuity was observed to be dependent on the thickness of the underlying Pd film. Substrates with $70 \mathrm{~nm}$ of Pd afforded films with a non-obvious interface between $\mathrm{Cu}$ and Pd as observed by TEM. A graded interface with intermixed $\mathrm{Cu} / \mathrm{Pd}$ phases was suggested. Substrates with $<10 \mathrm{~nm}$ of Pd afforded films similar to Ru substrates. A self-limiting growth rate of $0.45 \AA /$ cycle was confirmed at $220^{\circ} \mathrm{C}$. Arguably, the growth rate was stable between $190{ }^{\circ} \mathrm{C}$ and 250 ${ }^{\circ} \mathrm{C}$, although a growth rate vs. temperature plot showed a steadily increasing growth rate from 0 to $1.3 \AA$ /cycle between $150{ }^{\circ} \mathrm{C}$ and $350{ }^{\circ} \mathrm{C}$ on thick Pd films. Considering a recent 
report describing the role of Pd films in thermal Cu ALD employing molecular hydrogen, ${ }^{7}$ it is possible that the observed variable growth rate was an effect of variable hydrogen uptake in Pd with temperature. A saturation curve for the hydrogen pulse or varying the purge time preceding the hydrogen pulse would help to investigate this possibility.

The interaction of the precursor with a copper surface was modeled with DFT calculations and concluded that chemisorption of the precursor likely occurs through dissociation of the $\mathrm{Cu}-\mathrm{NHC}$ bond. However, the $\mathrm{Cu}-\mathrm{NHC}$ bond was calculated to be very strong at $293 \mathrm{~kJ} / \mathrm{mol}$. It is the nature of diamino carbenes to bond strongly to metal centers, therefore it is not possible to chemically modify the carbene to ease bond dissociation and assist precursor chemisorption. Steric factors of the carbene are changeable and maximising access to the central copper atom by lowering the steric bulk of the precursor is a valid direction for future work. Low temperature PE-ALD experiments for compounds $4-9$ in Chapter 7 involving a variety of potential barrier/adhesion layers would contribute to the understanding and usefulness of these precursors.

\subsection{References}

1 Whitehorne, T. J. J.; Coyle, J. P.; Mahmood, A.; Monillas, W. H.; Yap, G. P. A.; Barry, S. T. Eur. J. Inorg. Chem. 2011, 3240-3247.

2 Kim, T.; Yao, Y.; Coyle, J. P.; Barry, S. T.; Zaera, F. Chem. Mater. 2013, 25, 36303639.

3 Wu, J.; Li, J.; Zhou, C.; Lei, X.; Gaffney, T.; Norman, J. A. T.; Li, Z.; Gordon, R.; Cheng, H. Organometallics 2007, 26, 2803-2805.

4 Ma, Q.; Guo, H.; Gordon, R. G.; Zaera, F. Chem. Mater. 2011, 23, 3325-3334. 
5 Mandia, D. J.; Griffiths, M. B. E.; Zhou, W.; Gordon, P. G.; Albert, J.; Barry, S. T. Physics Procedia 2013, 46, 12-20.

6 Hagen, D. J.; Povey, I. M.; Rushworth, S.; Wrench, J. S.; Keeney, L.; Schmidt, M.; Petkov, N.; Barry, S. T.; Coyle, J. P.; Pemble, M. E. J. Mater. Chem. C 2014, 2, $9205-$ 9214.

7 Jiang, X.; Wang, H.; Qi, J.; Willis, B. G. J. Vac. Sci. Technol. A. 2014, 32, 041513 
